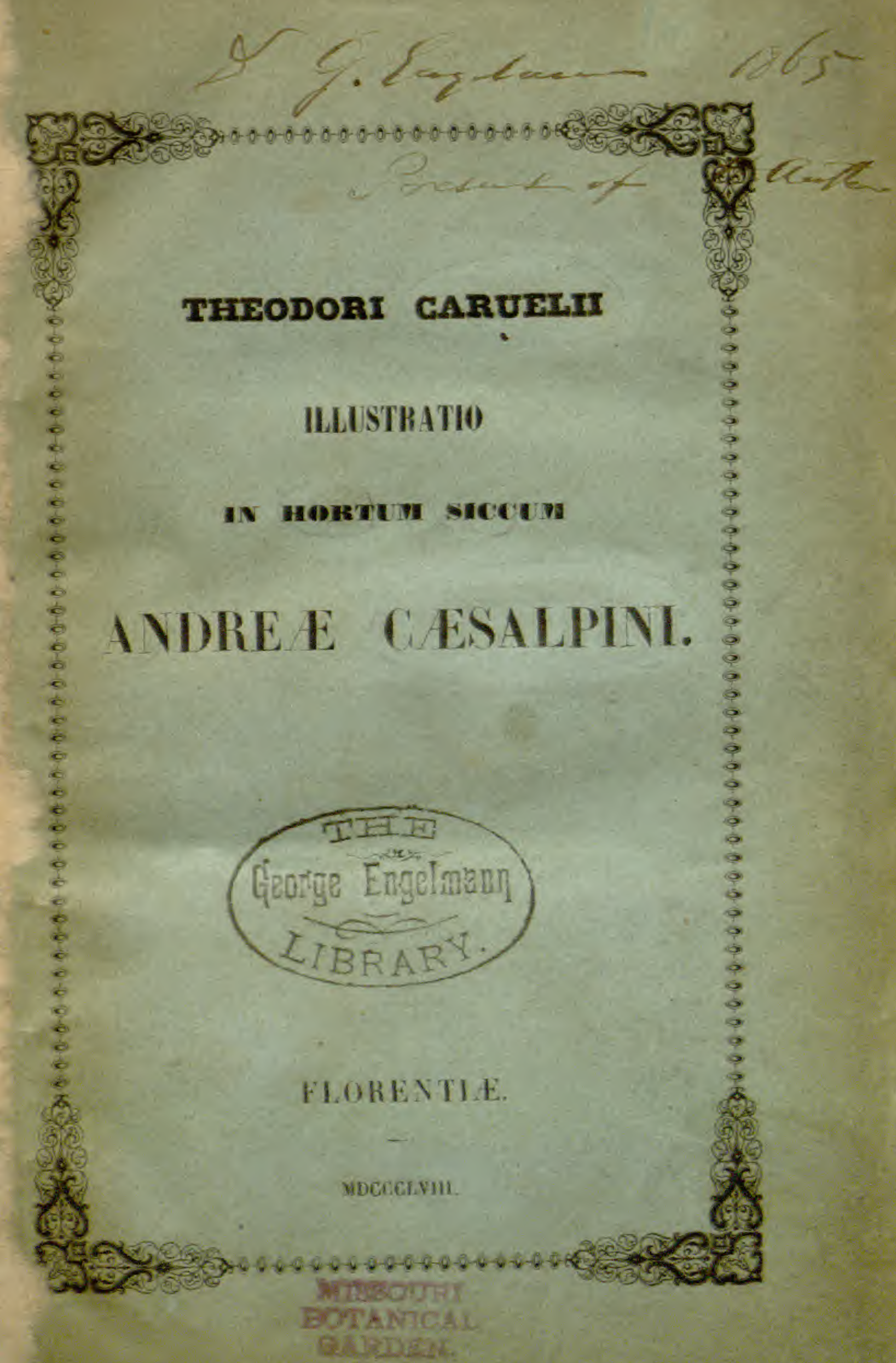




\section{- ILLUSTRATIO}

IN HORTUM SICCUM

ANDRE E CASALPINI. 
Corvel, 1.

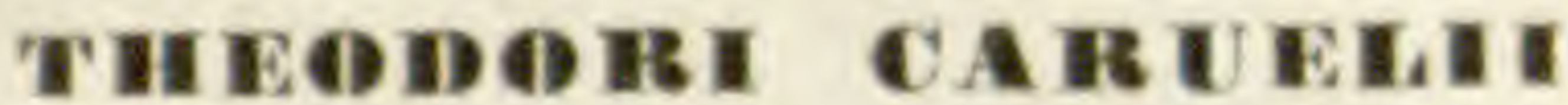

\section{ILLUSTRATI0}

IN HORTUM SICCUM

\section{ANDREE CESALPINI}

Rudimentum ex plantis libro agglutinatis vigere scio in testimonium corum, qux in hoe volumine a me dicuntur.

Cosaly. De plant. Preff. 
TUO - NOMINI - TUEQUE . MEMORIE

\section{CONSTANS . THEODORE . CARUEL}

PARENS . OPTIME . VENERANDE CUIUS - POTISSIMUM - CONSENSU - ET - OPE

NATURALIUM * DISCIPLINARUM * STUDIA

AB . IPSA A ADOLESCENTIA - EXCOLUI PRIMUM * HUNCCE

LABORUM . MEORUM * FRUCTUM PIO - gRatoque - ANimo

VOLENS - LIBENS - INSCRIBO 
DE HORTO SICCO

\section{ANDRE E GESALPINI.}

Ex præfatione quam Andreas Cæsalpinus præposuit operi suo De plantis inscripto comperitur a se quondam, adhortante maxime atque jubente Cosmo I M. D. Etruriæ, confectum Hortum Siccum; ejusque alterum exemplar Magno Duci, alterum autem fautori atque patrono suo Alphonso Tornabono antistiti Burgensi oblatum a se atque donatum fuisse. Primum vero exemplar post tot variisque temporibus effectas indagationes adhuc desideratur, neque vestigium quidem superest uspiam.

Alterum benigniorem expertum est fortunam, quippe quod Tornabonorum hæreditate in Pandulphinorum gentem, viros florentinos, commigratum, in domestica eorum bibliotheca ignoratum latuerit, donec Petrus Antonius Michelius post diuturnas investigationes susceptas ob Will. Sherardi legati Smyrnis pro Britanniæ regina instigationes (ut ipse tradit Michelius in commentariis quos manu scriptos reliquit) invenit ann. MDCCXVII. Certiorque factus Michelius de Herbarii pretio novissime atque felicissime reperti, ad plantas diligentius distinguendas, adpositis quæ tunc 
vigebant Tournefortianis nominibus, animum advertit: illustrationemque qua ille pollebat doctrina brevi tempore confecit. At opus, quod vir præstantissimus edendum sibi proposuerat, non fuit tunc, nescio quibus caussis, emissum; postque Michelii obitum cum universis ejus collectionibus in manus discipuli sui Joannis Targionii devenit, qui scriptum magistri et recensuit et adnotationibus non quidem textu indignis locupletavit intra annos, ut suspicatur, MDCCXXXVII atque MDCCXXXVIIJ.

Exinde Hortus Siccus Cæsalpinianus oblitus delituit usque ad ann. MDCCCXVIII; quo ex Octaviani Targionii Joann, filii requisitionibus in ædibus Nencinorum hæredum Pandulphinorum repertus est; atque paullo post in usum bibliothecæ palatinæ M. D. Etruriæ acquisitus fuit. Eodem quoque anno naturalis historiæ cultor Brocchius, qui tunc Florentiæ degebat, brevem reperti herbarii notitiam in Bibliotheca italica (vol. X, pag. 203) edidit: posteriore autem anno prof. Bertolonius domo Bononiæ commentarium de eo protulit, cui titulus Memoria sopra l'erbario e una lettera del Cesalpino. Interea Octav. Targionius qui tunc manu scriptum Michelii opus tenebat, ad illud edendum intendit animum, definitis nova ratione plantis, nominum Linneanorum appositione, ita ut Micheliana illustratio scientiæ ulterius progressæ se æquaret: at opus adgressus, primis lineis tantum designavit.

Anno tandem MDCCCXLIV Hortus siccus cæsalpinianus translatus erat e bibliotheca palatina in R. Florentinum naturalis historiæ Musæum, postulante 
prof. Phil. Parlatore, qui facultati botanicæ præfectus nuper in Musæeum arcessitus erat. Et ei quidem in mentem subiit horti cæsalpiniani illustratio: at vir præstantissimus aliis distractus curis non perfecit quod mente inchoarat.

At, ut nunc de antiquitate ejus aliquid tangam, inter cognitos adhuc antiquior certe, nisi fortasse antiquissimus, extat hortus siccus cæesalpinianus; quippe qui ad ann. MDLXIII referatur. Unus forsan hortus siccus cujusdam Greaultii chirurgi lugdunensis, a Jussieuis quondam in suis collectionibus et nunc Parisiis in Horto regio servatus et ad ann. MDLVIII adjudicatus, primum sibi locum ante nostrum vindicat. ${ }^{1}$ Posterior, Rauwolfianus qui est Lugduni Batavorum, confectus ex plantis a Rauwolfio in itineribus suis anno MDLXXIII ad MDLXXV collectis. Aldrovandianus qui præsto est Bononiæ, ad idem fere tempus cum cæsalpiniano adserendus, quum sit omnibus notum Aldrovandum coævum atque condiscipulum Cæsalpini fuisse; sed ad tempus certius affirmandum desunt argumenta. ${ }^{2}$ At insequitur procul dubio aliquanto posterior qui Basileæ servatur hortus siccus Bauhini, confectus, ut colligi potest, inter annos MDLXXVI circiter et MDCXXIII ${ }^{3}$

Anno MDCCCXLIV quum ex bibliotheca palatina ad historiæ naturalis Musæum transiit, Hortus siccus cæsalpinianus volumine constabat charta pergamena colligato. Tunc plantæ, jam aliquid detri-

Doctor Puel domo Parisis, in litt.

Prof. Jos. Bertolonius Bononiæe, in litt.

Prof. Meisner Basilex, in lit.. 
menti a tarmetibus passæ, cura prof. Parlatoris veneno infecta, omnes alias læsiones effugerunt: atque herbarium, jussu illius, interjecta chartæ plagula inter singulas paginas, servato tamen pristino ordine, tribus voluminibus rubra pelle circumtectis ligatum fuit. Et quamvis iis de quibus supra memini detrimentis affectum sit herbarium, plantæ tali sunt conditione ut plerumque certissime agnoscere possis. Parviores, radice exceptá aliave subterranea parte, integræ sunt; majorum adest pars superior tantum, florifera vel fructifera, sæpe cum aliquo vel inferiori vel radicali folio; interdum, si maximus sit, unus tantum flos. Aliquot specimina, pauca quidem præ aliis et plerumque eorum vel arborum vel fruticum qui faciliter et sic recognoscuntur, flore et fructu carent. $\mathrm{Si}$ animum altendas, statim videas plantas, quibus confectum herbarium, ex variis Hetruria regionibus, alias etiam ex hortis, collectas esse. Herbarium constat chartis CCLLX -magnjtudine ut vulgo dicitur in folio, omnibus numeratis, et plantas DCCLXVII complectitur. Specimina sunt chartis conglutinata, aut singulatim, aut plura secundum magnitudinem conjuncta. Inscripta sunt apud plantas græca latina atque italica nomina ex propria Cæesalpini manu, quatuor exceptis. Ad seriem notandam subjacet numerus ex alia manu et quidem posteriori, illius certe, ut puto, amanuensis quo Michelius utebatur; at ordo numerorum non semper rationi congruus : neque ipse admisisset Cæsalpinus. Primam herbarii frontem tenet italica epistola, magni quidem ad scientiam pretii. quam Cæsalpinus manu sua Alphonso Tornabono 
Episcopo inscribit. Proponit in ea quod de describendis plantarum classibus cogitaverit; ait velle se in hoc herbario methodum suam primis lineis designare, methodum quam post viginti studiorum annos perfectam scientiæ classico De plantis libro impertitus est; deinde monet aliquid de ratione qua plantas distribuerit et nomina adjecerit. Duo epistolam subsequuntur indices, græcus alter, alter latinus atque italicus: horumque numeri ad chartas herbarii remittunt.

Ego autem in Cæsalpiniano horto sicco illustrando hanc tenui rationem. Epistolam primum atque indices edidi ad exemplaris tidem quantum re typographica sineretur, ordinem quidem atque orthographiam notasque distinctivas autographi referens. Illustrationem aggressus singularum plantarum, illas eodem quo herbario inscriptæ sunt numero descripsi: præterea, plantæ quæ in singulis chartis prima tenet, numerum chartæ cui adtinet addidi. Nomina plantis inscripta, aliquot quidem non carentia erroribus, omnino ad fidem exemplaris, quantum in me esset, transcripsi. Ex opere De plantis loca citavi, quibus aut certe aut probabiliter planta describitur: quumque in illo opere alia inhæreant nomina plantis, et hæc addidi. Quo modo plantarum specimina se habeant, monui, an florifera an fructifera an integra etc. Denique nomina plantarum recentiora adjeci, suisque synonimis vel, ubi opus esset, animadversiunculis citationibusve declaravi.

At nunc producenti mibi in lucem hoc opus, quod non exiguo labore confeci, nec spes quidem subvenit 
par emolumentum scientiæ adferendi: quum præsertim talia intercedant tempora, quibus et historiæ botanicæ studia obsoleta, et perpaucis sit curæ vestigia mente relegere relicta a patribus in hac via qua posteri contendimus, vel exquirere quomodo ipsi cognitiones has abripuerint quas nobis servandas atque augendas tradiderunt. Reputantem autem opus meum et illis usuventurum qui geographiæ botanicæ studio quæsierint quomodo temporibus cæsalpinianis vegetatio etrusca se haberet, et illis qui specierum historiam indagaverint, et illis maxime qui in opus De plantis difficile lectu sine hac illustratione incubuerint, non pcenitet me quidem laboris in opere impensi. Immo in hoc maxime lætor, quod ego viro præstantissimo, botanices philosophorum primo, id, quod Michelio aliisque præclarissimis concupientibus fortuna denegavit, hoc quasi observantiæ tributum solvere possim. 


\title{
D. O. M. A.
}

AI Ro $^{\text {mo }}$ Monsignore il $\mathrm{S}^{\text {or }}$ Alfonso veschovo de Tornabuoni

\author{
Patrone, \& Compar' mio oss ${ }^{\mathrm{mo}}$
}

Se bene il numero delle piante Monsignor' $\mathrm{R}^{\mathrm{m}}$ cresce quasi in infinito, \& questo dal' intelletto humano non si può comprendere: nondimeno con il raccorne molté in una simiglanza, \& ridurle in questo modo a breve numero, facilmente se ne può haver' quella cognitione, che a noi s' aspetta. Questa riduttione fu tentata da alcuni degl' antichi, da Theophrasto principalmente, il quale con gran' diligentia esaminò gl' alberi, le biade, \& gl' hortaggi : ma circa l' herbe medicinali se ne passò leggiermente. Dioscoride poi di tutti gl' altri piu copioso, havendo risguardo alla Medicina, ridusse insieme quelle che hanno simiglanza nelle virtu, \& le separò da quelle che in ciò sono dissomiglanti: Con il quale ordine ha irricchito la Medicina de rimedii, quanto hoggi è impoverita dal' commune uso de nostri medici, i quali per non durar'fatica, contentandosi di poche cose, con la cicorea \& con la Buglossa \& con e discorsi fatti in camera medicano ogni infirmità. Aleuni altri hanno raccolto cio che hanno trovato scritto de Semplici, ma senza ordine, da quali non si può cavare altro che una historia assai eonfusa \& molto difficile anzi impossibile a mandarla a memoria. L' ordine adunque di Dioscoride serve solamente per quelle piante, de quali si sanno le virtu : ma per 
hạvere una general' cognitione di tutte, non è suffitiente, percioche è impossibile haver' havuto esperientia di tutte quelle, che ci si rappresentano di nanzi. Però la dottrina di Theophrasto in questo è molto a proposito; il quale considerando le differentie secondo la faccia di ciaschuna pianta, \& secondo le parti toro, facilmente raccogle quelle che sono d'una medesima schiatta. Ma per non haver' egli eseguito tale ordine se non in poche cose, avengha che pare si sia contentato solamente d' haver' dato il modo, \& non essendo infino a qui stato alcuno, il quale si sia messo a questa impresa, mi è venuto nell'animo di provare, se in ciò il mio picciolo ingegno potesse cosa alcuna. Ne mi ha tolto l'animo il vedere molti eccellentissimi huomini di questa professione infino a hoggi essere stati, \& nessuno haver' tocco questa parte: Percioche fra e Semplicisti pochissimi sono quelli che habbino congiunto questa professione con li studii di Philosophia; senza la quale non è possibile farvi frutto alcuno: $\&$ fra e Philosophi di qualche conditione, pochissimi sono che habbino messo e loro studii nelle cose particolari, come sono e Semplici, anchorche tutto il nervo della Philosophia consista in quelli. Hora se io barò fatto in l'una e l'altra professione tal' profitto, che basti a mandar' a effetto il mio proponimento, ne ringratiarò Iddio, \& quelli che in cio mi hanno aiutato, fra quali è V. S. R ${ }^{\mathrm{ma}}$ alla quale resto sempre obligatissimo. Et per dar' principio a quest' impresa, ho fatto come quel' sonatore, il quale innanzi che cominci la sonata, fa prima una ricercata, per vedere se nessun' tasto vi è che scordi. Però essendomi messo innanzi tutti e semplici, quali infino a qui mi sono venuti alle mani, gli 
ho distribuiti per questa prima volta grossamente, facendoñe le schiatte separate l' una dall' altra secondo il mio primo proponimento: \& desiderando V. S. $\mathbf{R}^{\text {ma }}$ che io gli facessi una ragunata de semplici ataccati sopra e fogli per riconoscerli, quelli de quali ho possuto haverne il saggio, ho atacchati in questo libro secondo quell'ordine. Et per dar'ne alpresente una breve dichiaratione, è da considerare, che non secondo la simiglanza delle fogle, ne de fiori, ne de semi, ne delle radici, ne d'altre simili parti sono le piante d'una medesima schiatta : ne anche per esser' in quelle dissomiglanti, sono di diversi generi: il che facilmente discorrendo si può vedere, avengha che grandissima dissomiglanza è fra le fogle dell' Elleboro nero, ¿ le fogle del bianco, similmente infra le fogle della lattuga domestica \& quelle della salvatica, non dimeno sono d' una medesima schiatta. Et per il contrario, de Ranunculi uno ha le fogle simili al' Appio, un' altro simili a quelle del finocchio, \& uno ven' è che ha le fogle di Grano: non dimeno tutti quei Ranunculi fanno una schiatta, \& quell' altre sono lontanịssime. Il medesimo si può considerare uelle simiglanze \& dissomiglanze de fiori, de semi, delle radici, de fusti, \& altre parti. Et ciò ragionevolmente aviene, perche la perfettion'delle piante d' onde dipende l' essere di ciaschuna, \& d'onde procedono varie generationi, anchorche non sia senza le dette parti, non però è in quelle, ma in quella sorte d'anima, qual' chiamano Vegetativa, la quale non ha altro offitio, che dar' la vita, \& mantenere la spetie: però tutte le parti gli sono state date per questi dui usi : Le radici per trarre il nutrimento, qual' mantiene, in vita: l'altre parti per generare altre simili a se, come 
il fusto, per portare il frutto, nel quale si contiene il seme: le fogle per coprire il detto frutto: i fiori sono uno degl' involti d'intorno a frutti teneri. Havendo adunque la natura variato quanto ha possuto, d' intorno alle radici non posseva molto variare, percioche tirando le radici il nutrimento gia nella terra preparato, come fanno le vene dal ventre, non hanno havuto bisogno di molti instrumenti per preparare il cibo, come fanno gl'animali. Ma gl' artifitii mirabili \& varii instrumenti appariscono in quella parte che serve alla generatione: dove pare che la natura si compiaccia sopra modo, con il mostrare al mondo le sue bell' opre vestite di varie fogle, ornate di vaghi fiori, \& in ultimo cariche di bellissimi frutti, con tanta varieta, che pare non si trovi fine d'intorno alla moltitudine delle spetie. Perche alcune mostrano fuori il lor seme quasi ignudo, come il finocchio, le Ferule \& simili : Altre lo tengono racchiuso in varie sorti d' involti \& di vasi, chi piu semplici, chi piu composti, chi soli, \& chi acompagnati in varii modi \& varie figure: Alcune altre come piu imperfette non par' che faccino seme alcuno ne fiore ne fusto, come il Capelvenero, la Felce, \& simili: le quali tutte fanno in cambio di seme una lanugine nella parte di sotto della fogla, nella qual' si contiene la virtu di rinovar' la pianta, come si è veduto nelle Felei, le quali son' nate dove sono state gettate le fogle. E funghi similmente non par' che faccino cosa alcuna per mantener' la spetie, non dimeno la parte tiglosa che sta sotto il capello è fatta per questo in quelli ne quali si trova : \& si assomiglono a quelli animali che stanno ataccati alle pietre, e quali per esser' imperfettissimi, non generano in loro concetto alcuno, ma un' 
certo escremento che da loro stilla, è suffitiente a nascere, come dice Aristotele dell Ortica marina, delle spugne, \& delle ostreghe. Adunque da e modi varii del produrre e semi, o quello che ba proportione con e semi genitali, \& dalla simiglanza di quelli ho rintracciato e generi \& le spetie delle Piante, conciosiache quelli più propinquamente mi dinotano la virtù dell'anima, per la quale tutte hanno l' esser' loro. Ma perche quivi si ricercheria una lunga dichiaratione, per sapere quali \& quanti siano questi generi, \& come siano multiplicate le spetie di ciaschuna sorte, non sendo hora commodo, mi riservo in altro tempo a farlo. Basta che per questa prima volta io gli ho distinti grossamente come dissi di sopra, però non sarà meraviglia, se alcuna pianta non harà havuto il luogo suo. Et ho ragunati gl' Alberi \& Arbusti tutti insieme, ma per adesso senza distinctione, quali ho messi nel primo luogo. Dipoi seguono quelle piante, che producono il seme nudo senza alcuno involto. Appresso vengono quelle, che I' hanno racchiuso ne vasi, distinguendole secondo la diversità di quelli. In ultimo ho messe quelle che non fanno seme qual' si conosca. Et d' intorno a ciaschuna pianta ho posto e nomi sua piu usitati appresso de Græeci, de Latini, \& de Vulgari, ponendo sempre nella parte superiore i Græci, \& nella piu bassa e Vulgari \& nel mezo e Latini, segnando e nomi græci con e charatteri græci, quando da Autori græci quelle ci siano state descritte o nominate: perche vene sono di molte che hanno il nome Græeco, ma l'Autore è Latino, come Plinio \& altri, \& questi ho segnati solamente con e charatteri latini. Et se nel nominar'le io in molto son' differente dagl' altri che hoggi ne fanno professione, 
hora non è tempo di renderne la ragione. Dove una pianta è presa per un' altra, io vi ho posto questo segno $p$ : Et di tutti i nomi per ritrovar'li facilmente, ne ho fatto dui tavole secondo l'Alphabeto, una de nomi græci, \& l'altra de latini insieme con e vulgari. Resta hora che io preghi V.S. $\mathrm{R}^{\mathrm{ma}}$ che insieme con questo piceiol' dono ella accetti il mio buon'animo, che ho di servirla, \& farli cosa grata. Et perche ciaschuno tien' chari e frutti sui comunche lor' si siano, desiderando che queste poche fatiche restino appresso di V. S. $\mathrm{R}^{\mathrm{ma}}$ accio alcune volte io le possi rivedere, farò come quel' testatore, il qual' volendo che la sua hæredita nella casa rimanghi in perpetuo, la lassa con questa conditione, che quei beni non si possino in modo alcuno alienare: cosi io conoscendo la liberalita di $\mathrm{V}$. S. $\mathrm{R}^{\mathrm{ma}}$ la voglo preghare \& quanto posso astrignere che facci che questo libro resti sempre in casa di quella, in memoria che io gli sono buon' servitore : \& a quella humilmente basciando le mani mi raccomando, quale Iddio prosperi \& mantenghi. Di Pisa, el di. 14. di Settembre. 1563.

Di V. S. $\mathrm{R}^{\mathrm{ma}}$ minimo $\mathrm{S}^{\mathrm{re}}$

Andrea Cesalpino: 
A Bporóvov. ....... 78

'A '́ńparov ........ 76

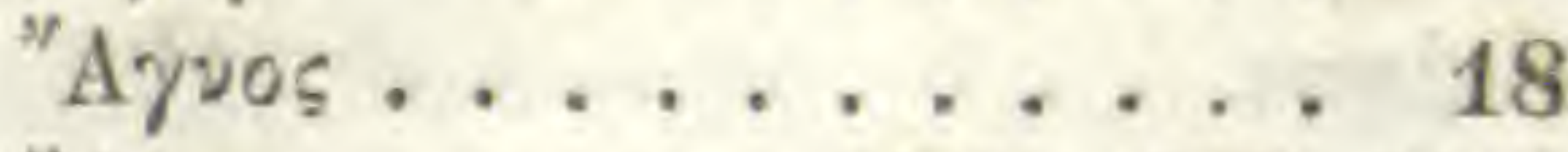

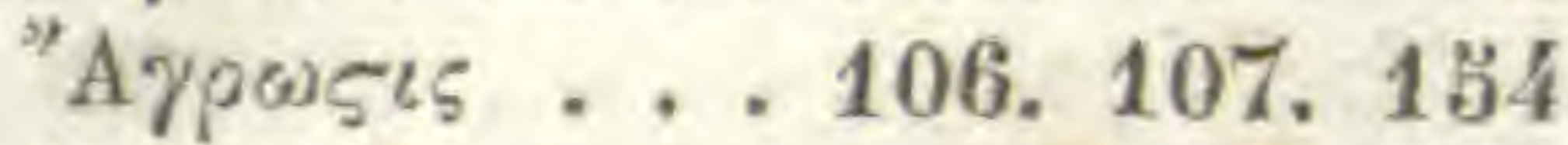

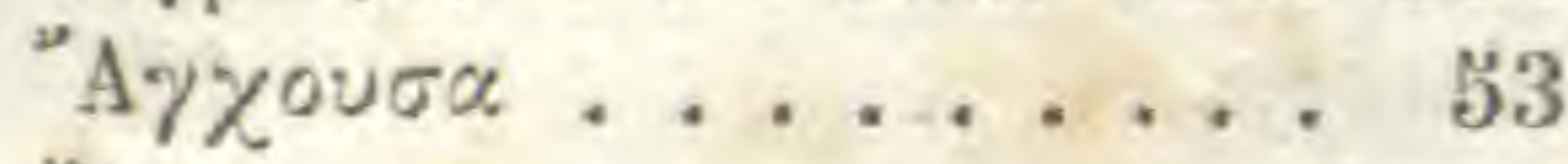

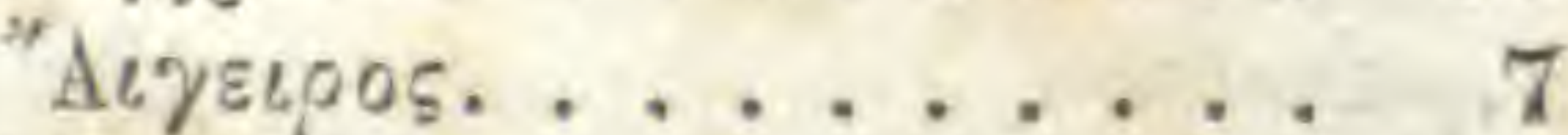

Atyó̀

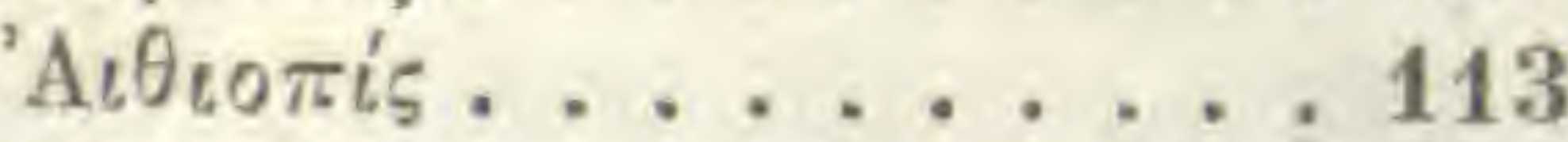

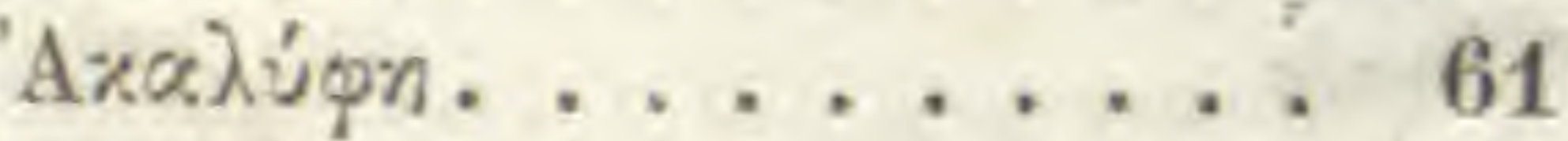

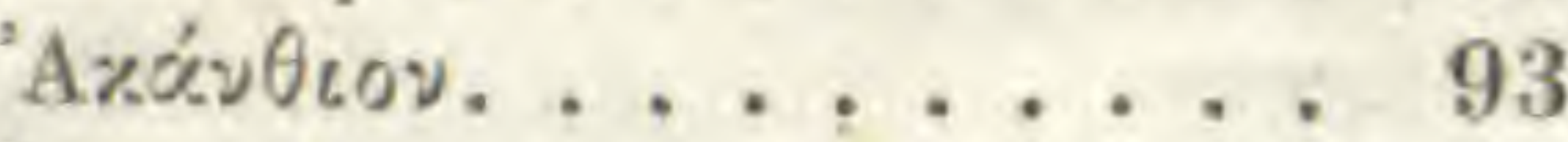

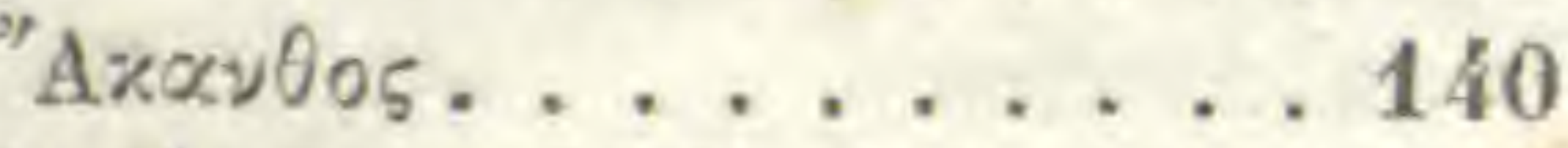

'Aหóvเтоข. 199. 200. 249. 250

"Azopvo . . . . . . . 94

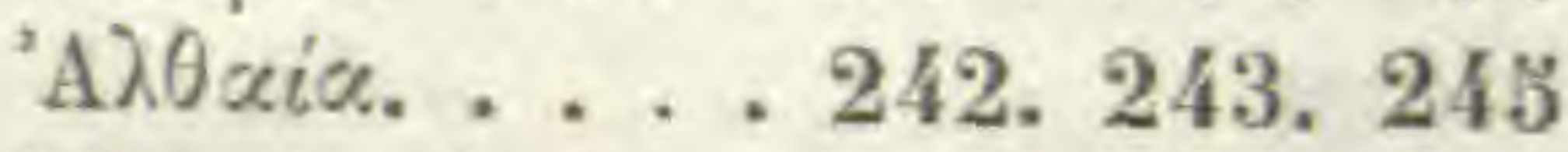

'Akexáxaßos . . . . 143

"A

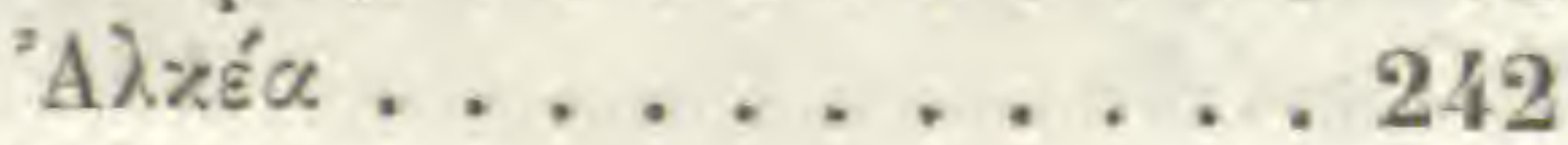

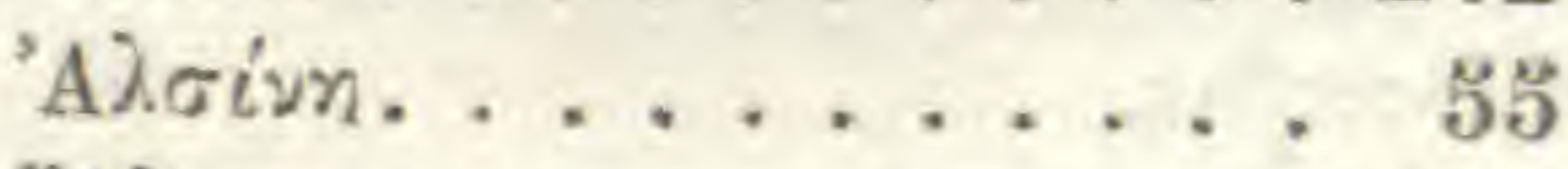

"A

"A Aveov . . . . . . . 196

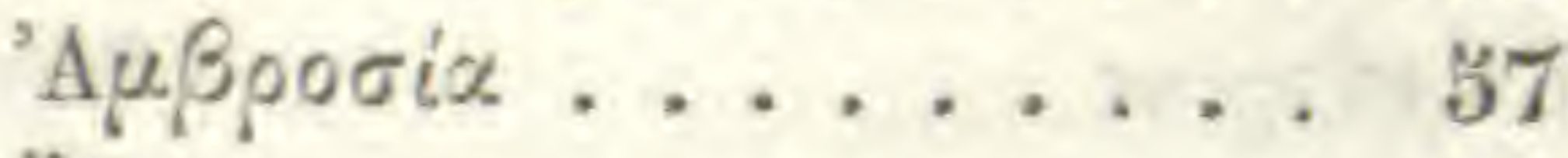

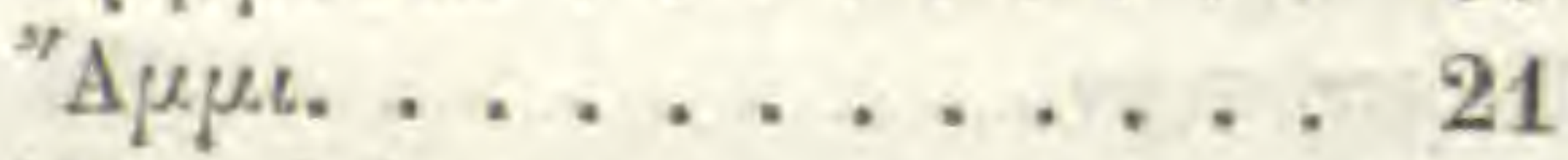

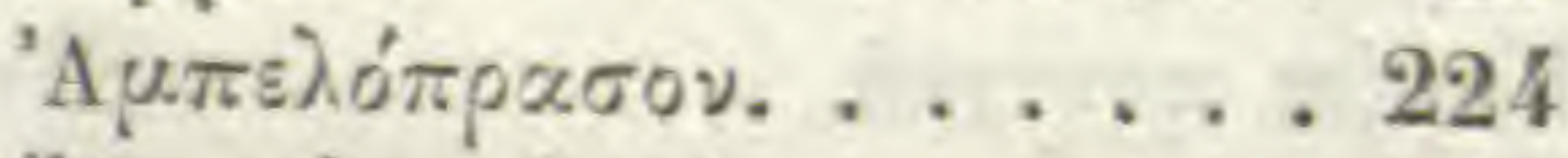

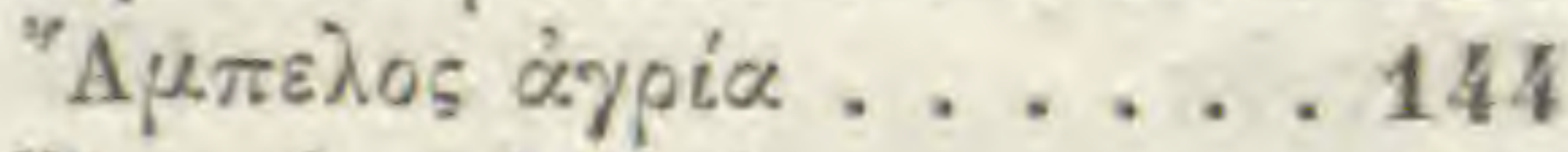

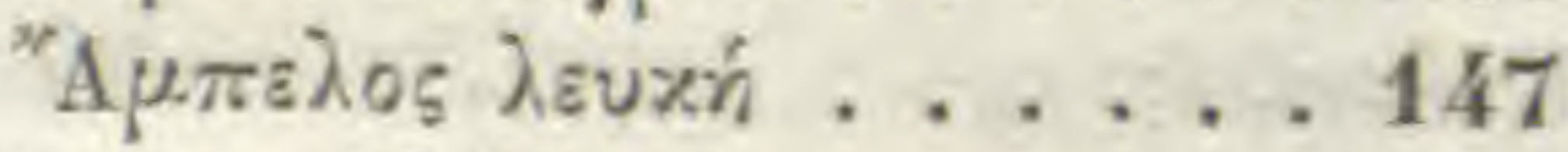

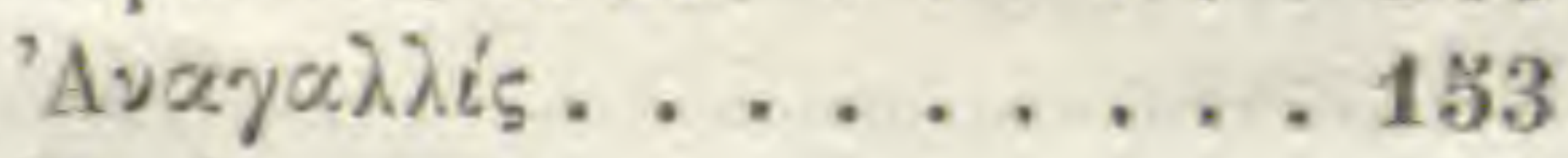

'Avćrypes. . . . . . . 13

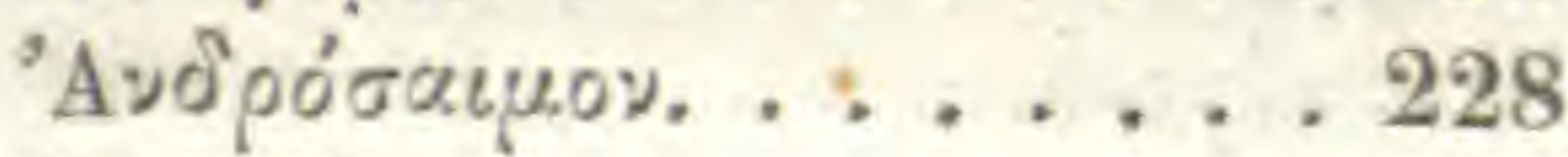

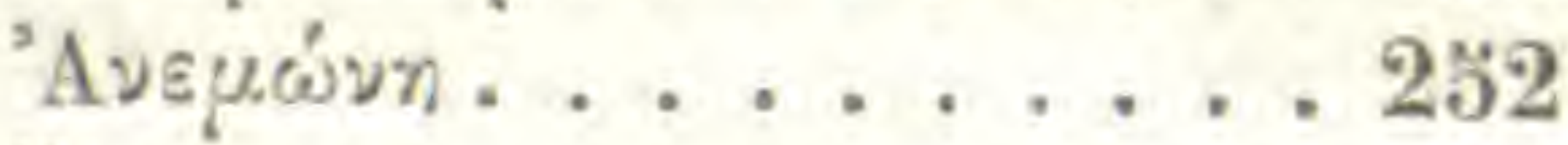

${ }^{* A \nu \theta \varepsilon \mu \iota \varsigma ~ \ldots . . . . . ~ . ~} 69$

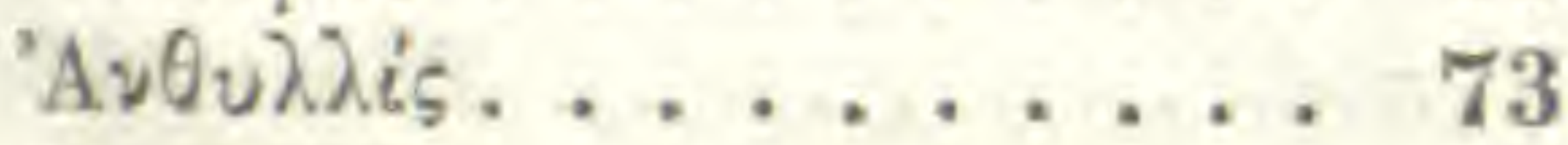

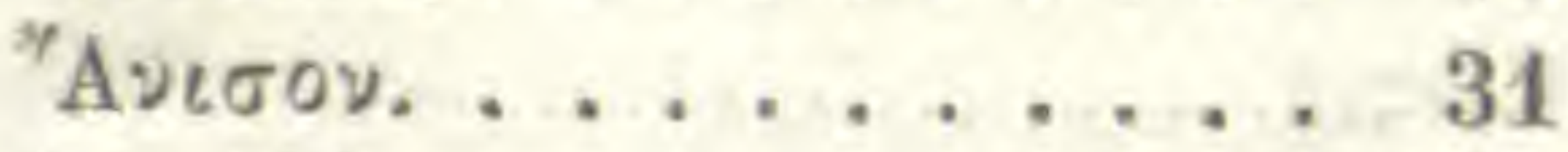

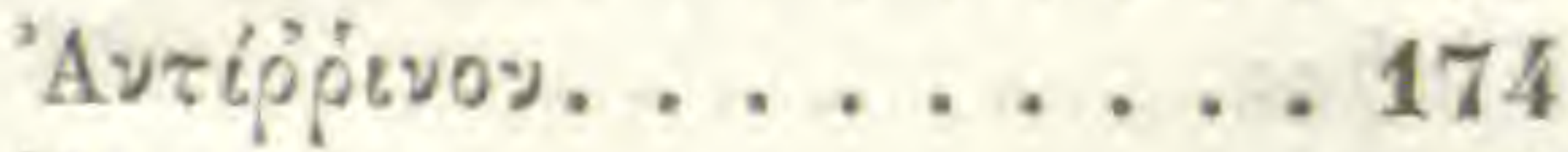

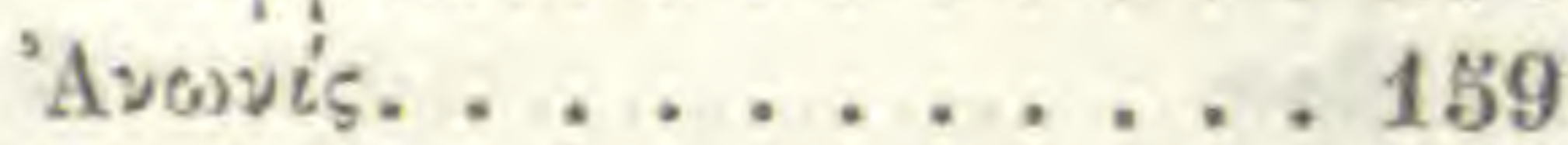

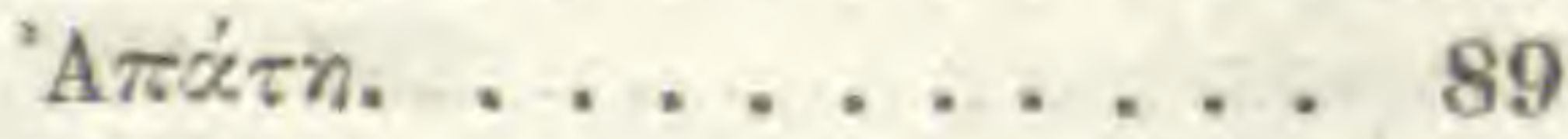

'Аสо์สบของ. . . . . . . . 186

'Apírapov... . . . . 141. 142

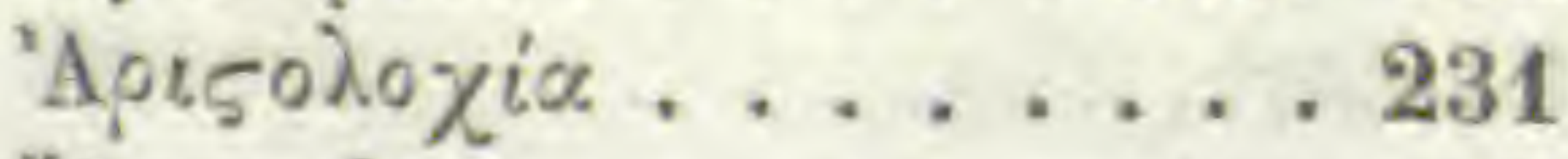

"A prev005 ......... 10

"А preto. . . . . . . . 102

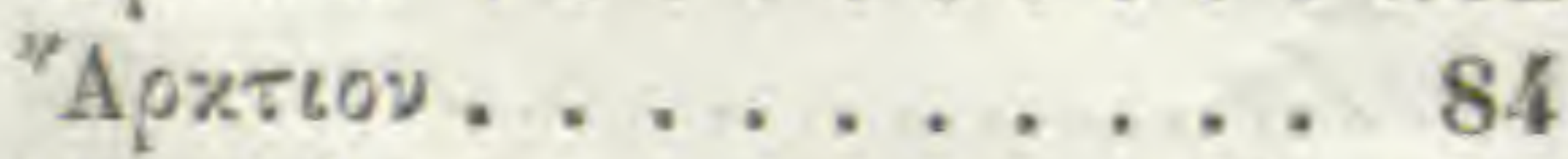

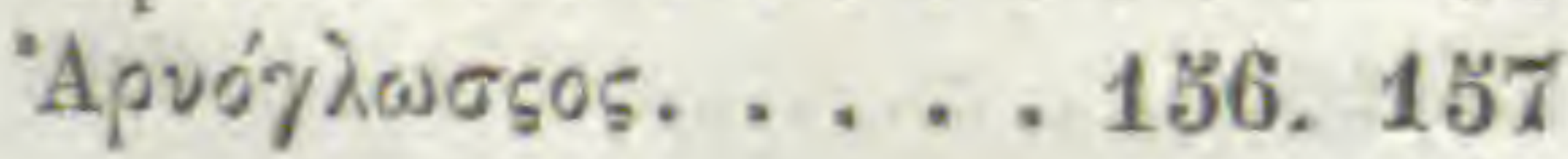

"Аро. . . . . . . . 141. 142

'Aอ́pระó์

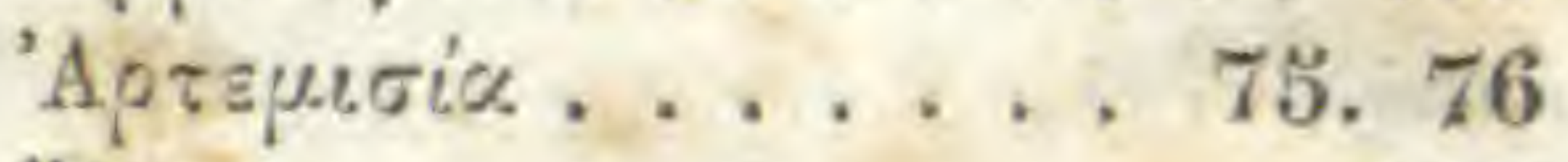
"Абкроч. . . . . . . . . . 229

"Абхироч. . . . . . . . 227

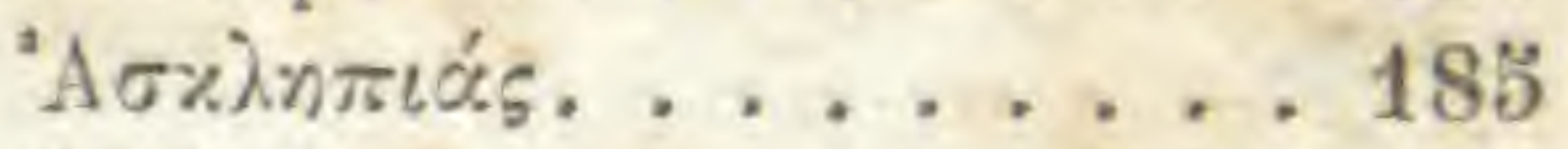

'A

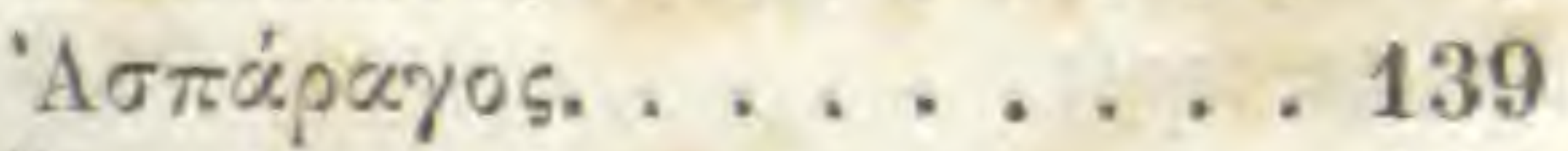

"A $\sigma \pi \lambda$ inขov . . . . . . . 264

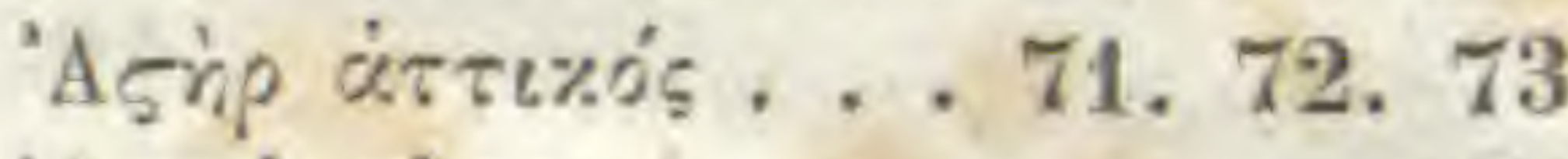

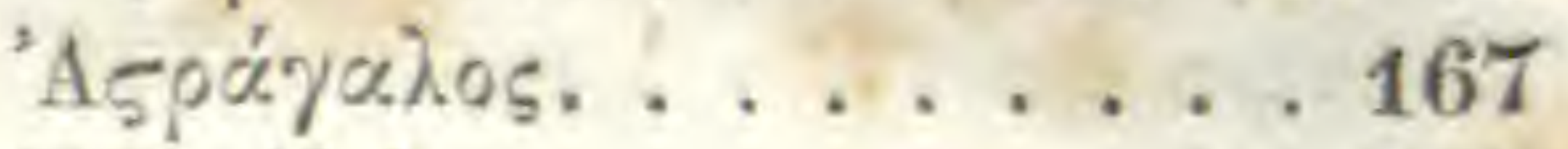

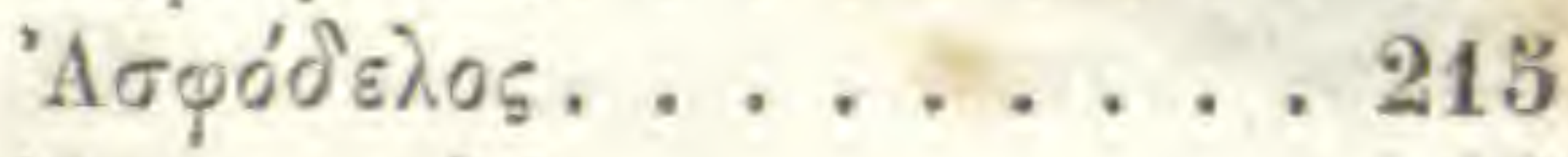

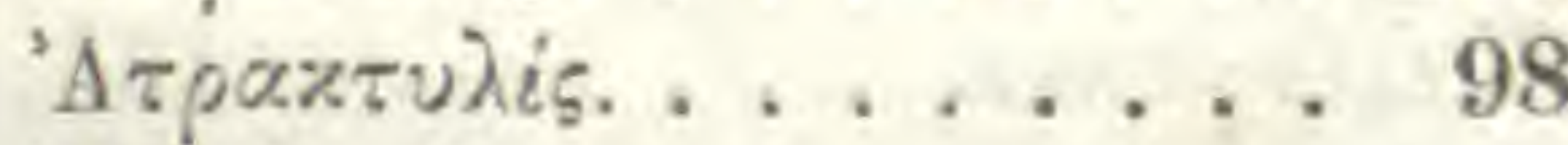

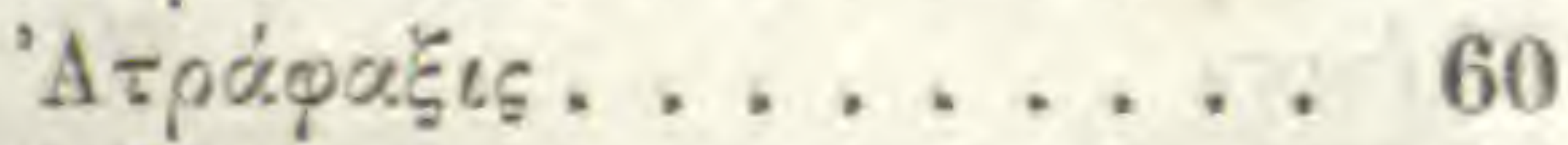

'A

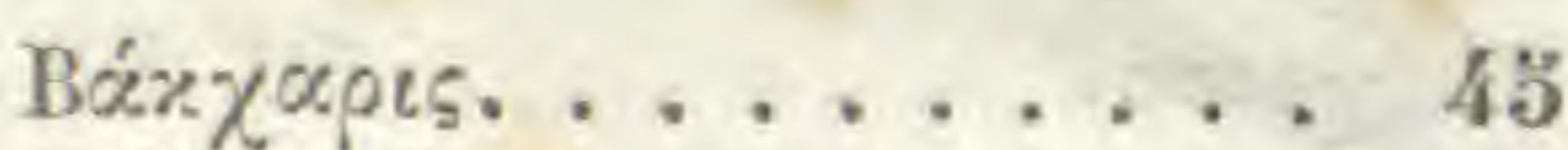

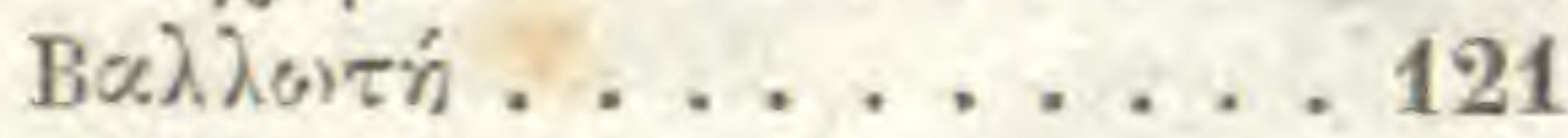

Búloos idocia ........239

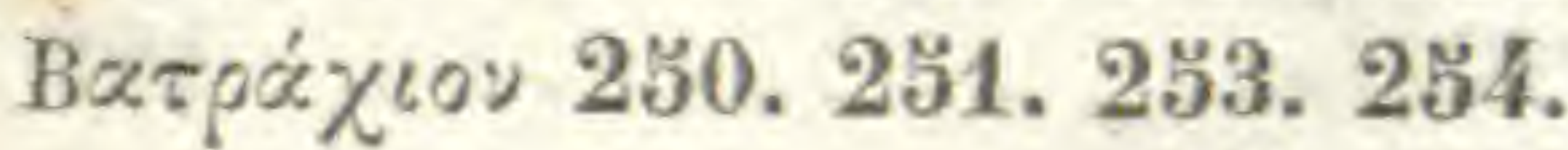

2รัว. 256

Bín $\chi^{\circ 02} \ldots \ldots \ldots .81$

B

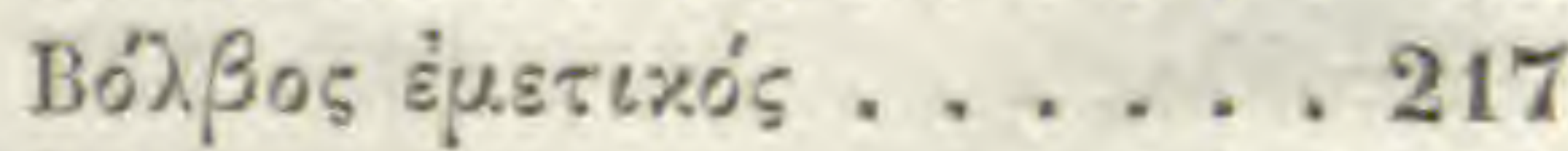

Bótpus. . . . . . . 5 57

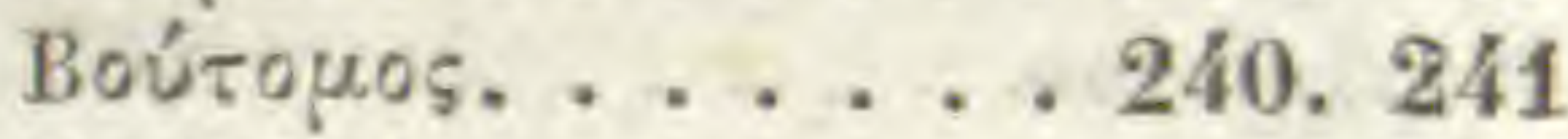

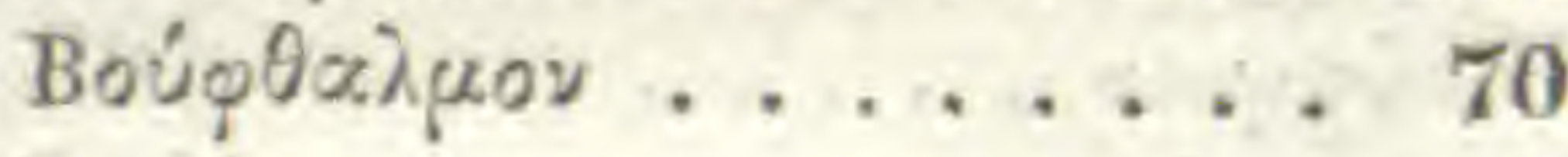

Bpd́tov . ........ 8

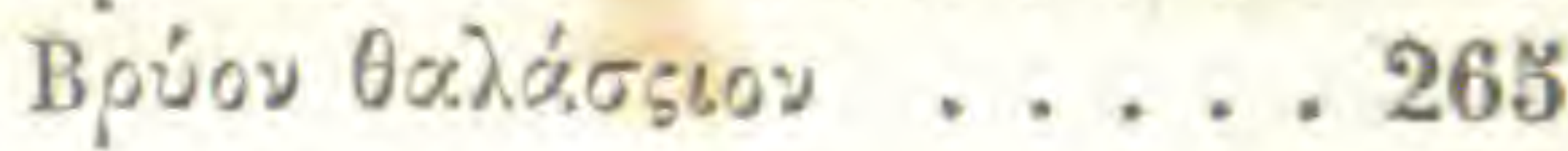

Гальочеs........ 172

$\Gamma \alpha \dot{\lambda} \lambda \operatorname{co\nu }^{2} \ldots . .2 .203$

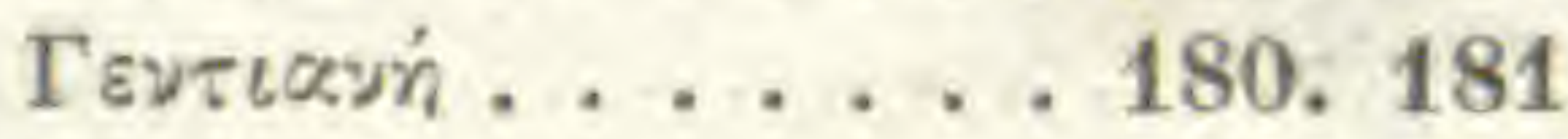

Гвра́ขtov . . . . . 36. 258

$\Gamma \lambda \propto u ́ \xi \ldots \ldots \ldots . .163$

$\Gamma{ }^{\prime} \dot{n}_{\chi 0 \nu} \ldots \ldots \ldots \ldots 127$

$\Gamma \nu \alpha \phi \alpha \lambda_{102} \ldots \ldots \ldots 154$

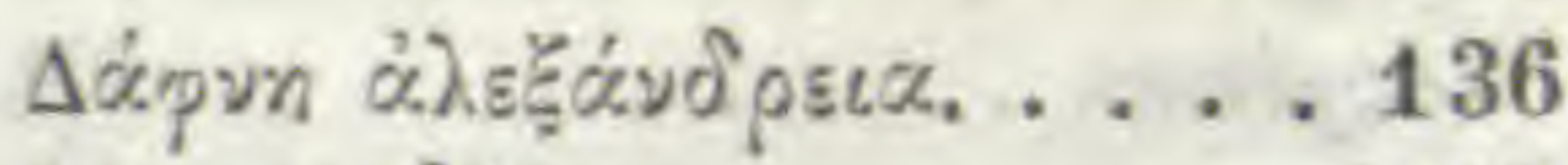

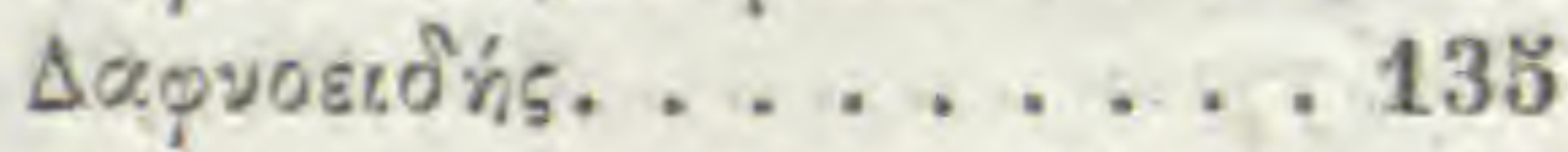
$\Delta$ aúxos. . . . . 32. 31. 34 $\Delta$ Eोipivio\%. . . . . . . 195 


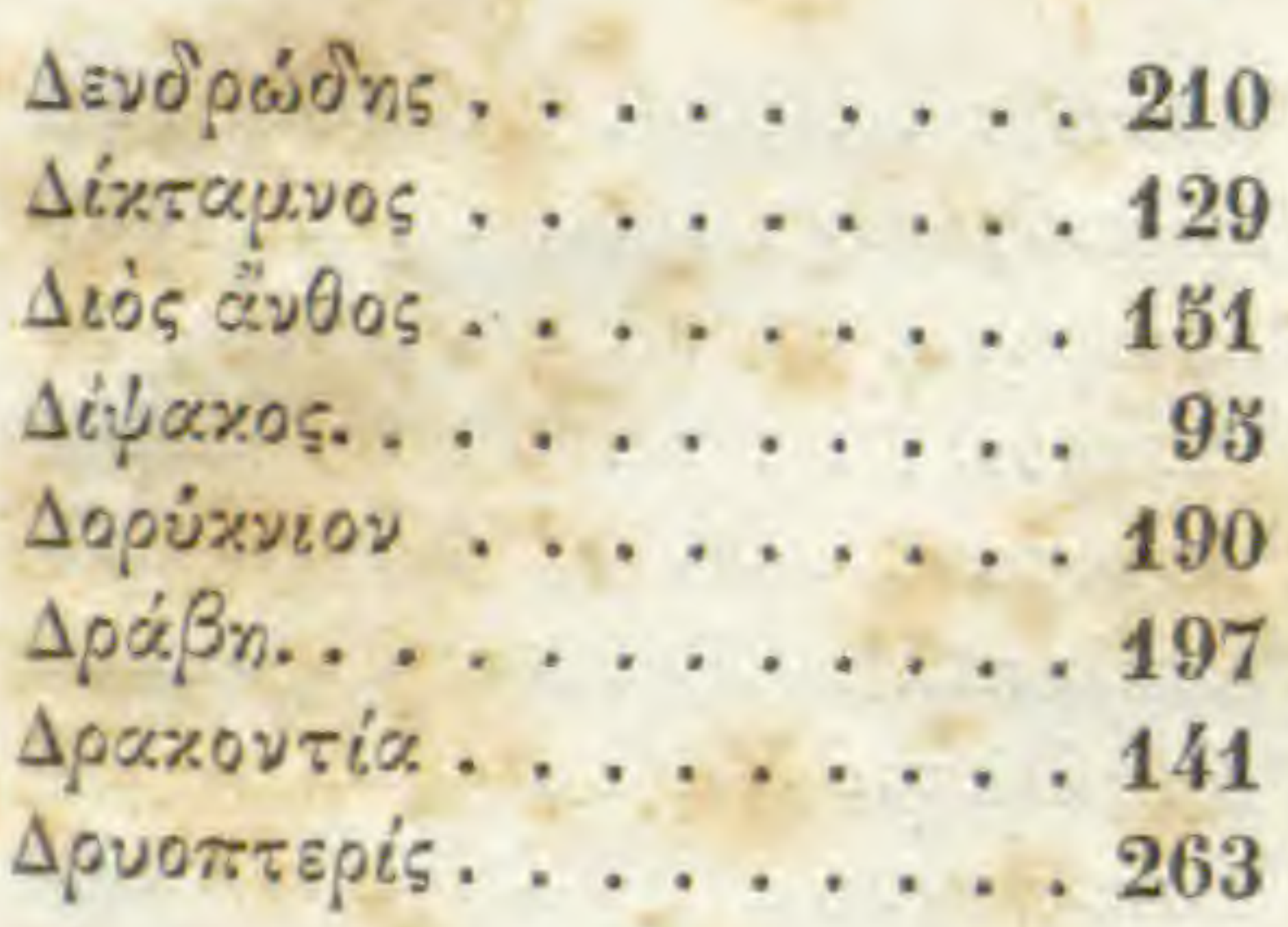

Èć̃ .......... 10

Eiarivn .........229

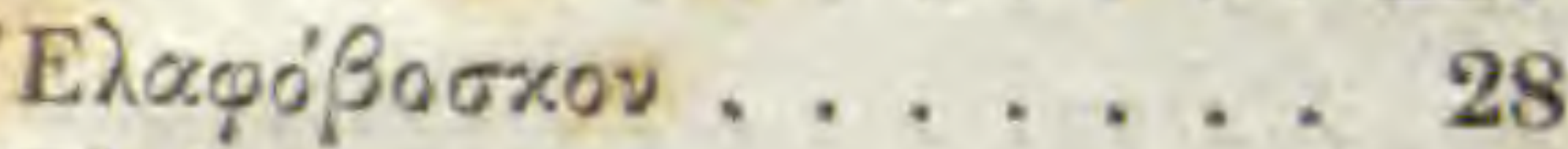

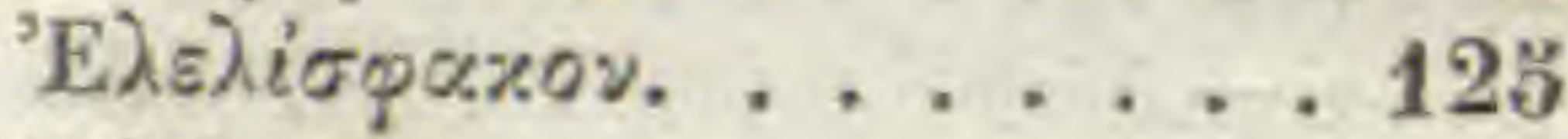

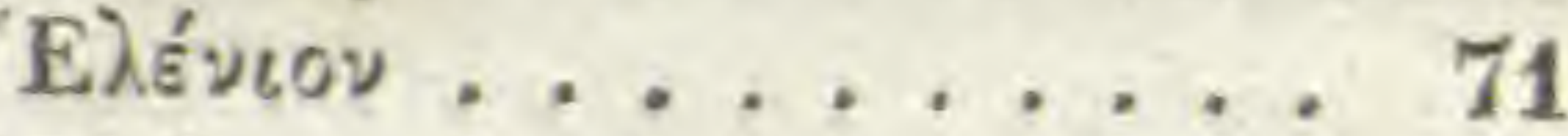

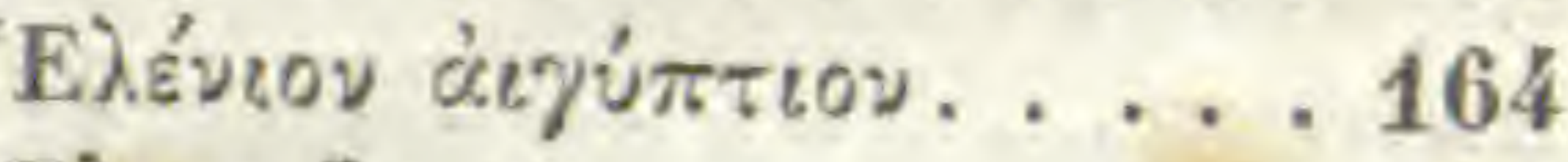

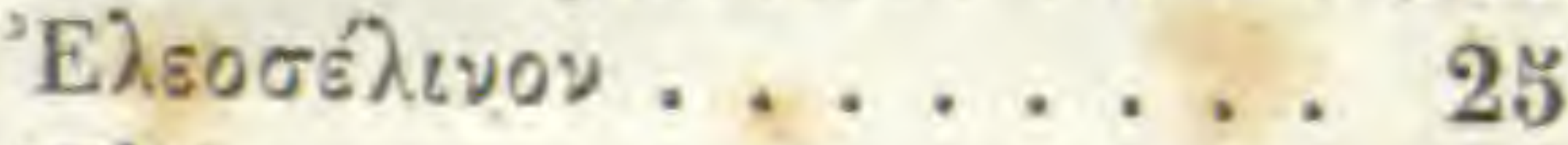

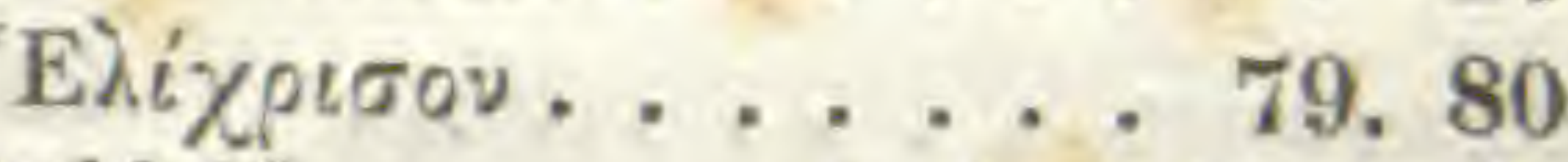

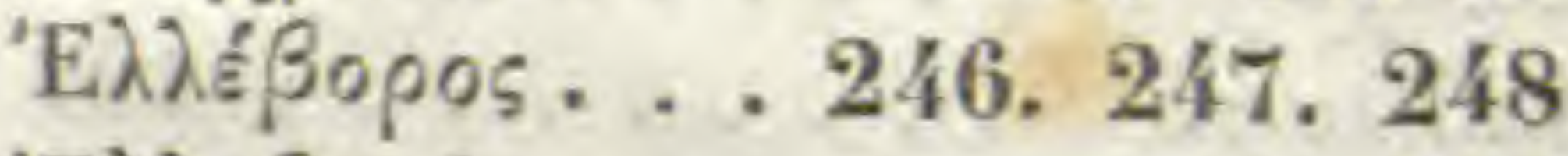

${ }^{\prime} E \lambda \lambda=$ Sopivn ....2.248

'E $\lambda_{\xi} i$ in . . . . . . . . 189

"Е

'Етьциं́

${ }^{2}$ Epsizn. . . . . . . 7.8

"Е $\rho \pi \nu \lambda \lambda \alpha_{05} \ldots . . .129 .127$

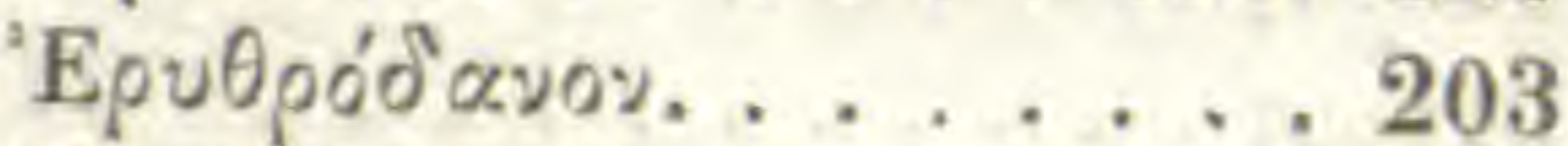

Eрv0рóvto\%. . . . . . 219

'Eрúqtног . . . . . . . . 193

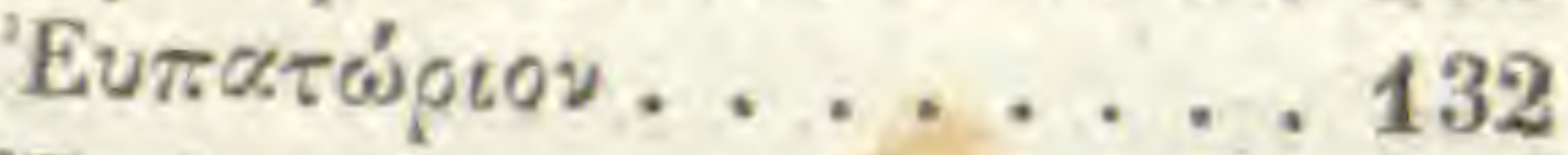

${ }^{2}$ Evésupos . . . . . . . 1

Eф̆íuврог . . . . . 221. 226

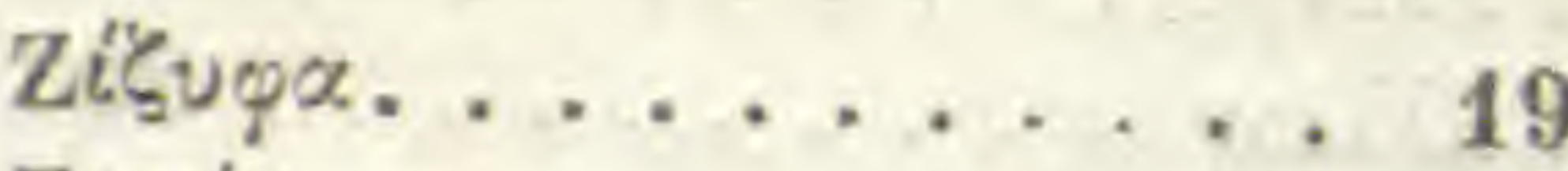

Zuvia........... 2

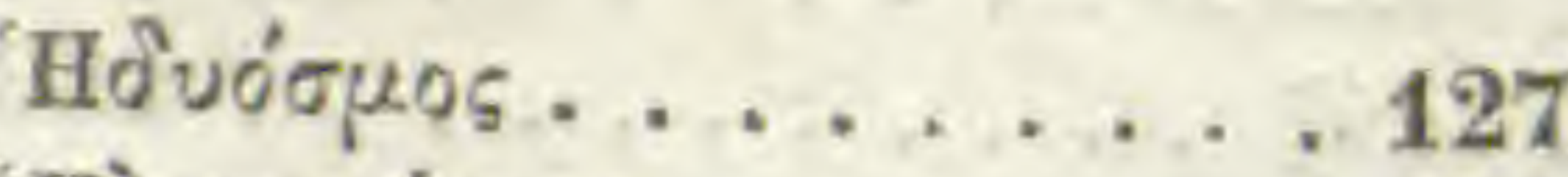

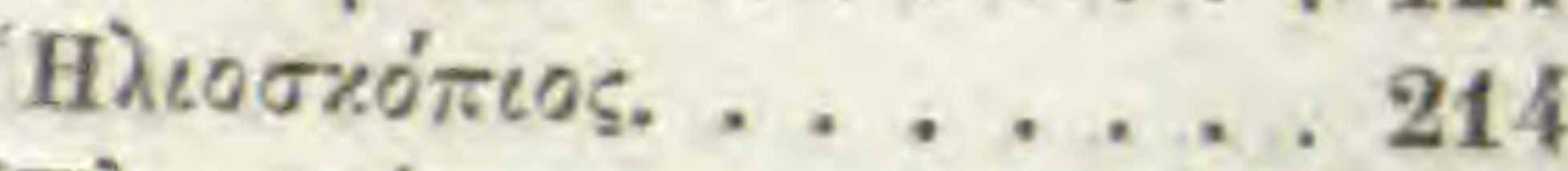

'Н Ниотро́тво2. . . 50. 182. 207

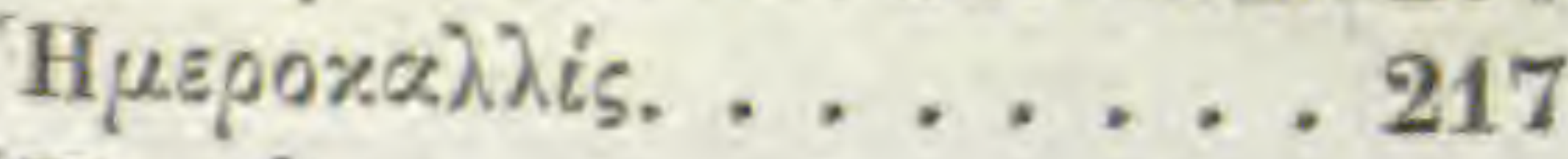

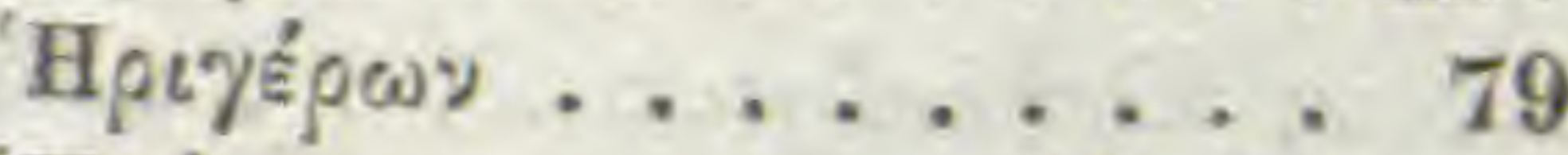

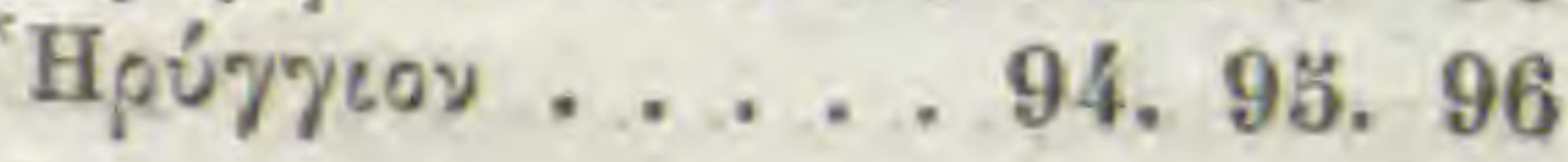

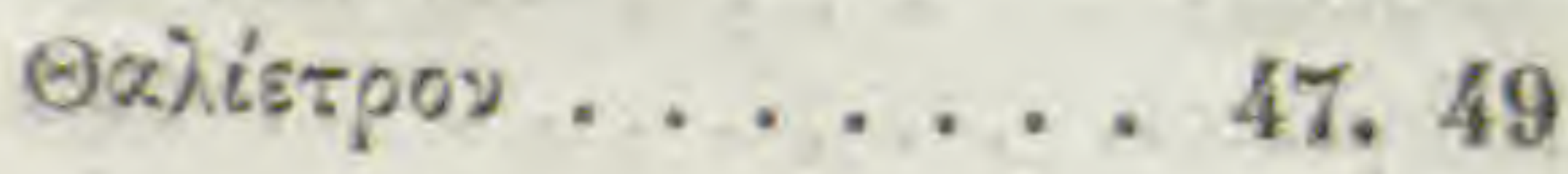

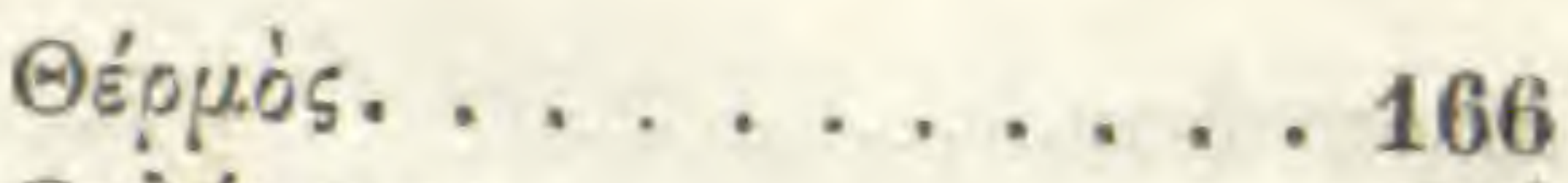

En

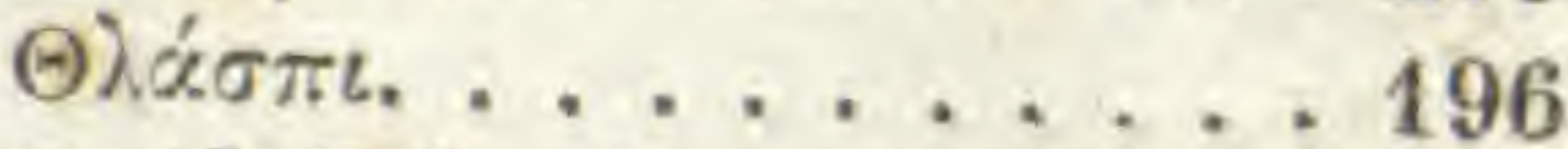

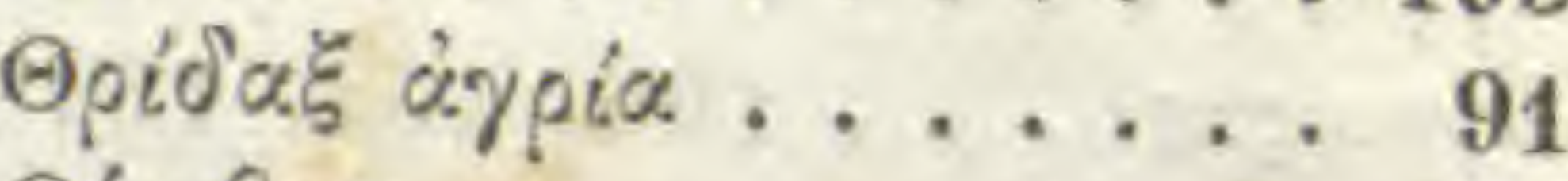

$\Theta \dot{u} \mu \beta p \alpha \ldots . . . . . .128$

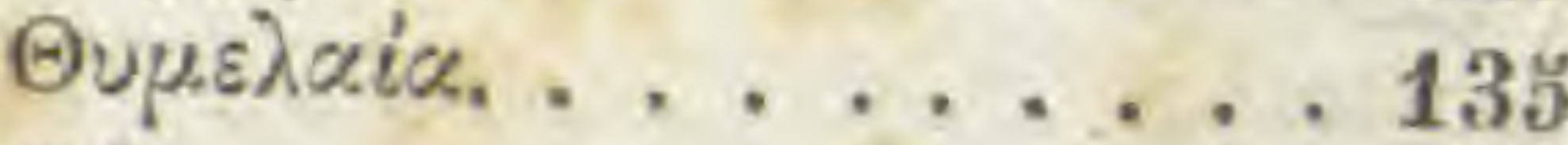

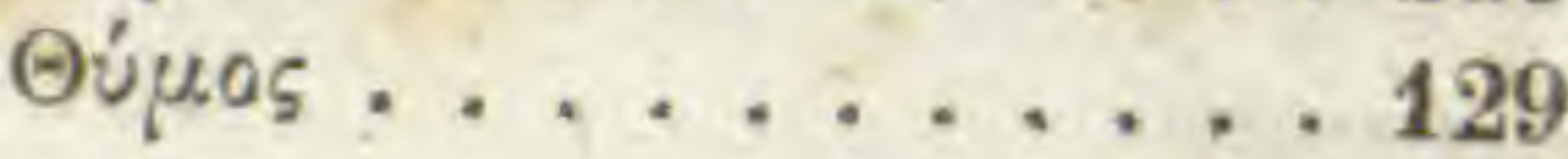

'T 1 spis . . . . . . . . . 198

'Iвpáxıoу . . . . . . . . 88

I $\pi \pi 0 \lambda_{\alpha} \pi \alpha \theta_{0 \nu} \ldots \ldots \ldots . \quad 59$

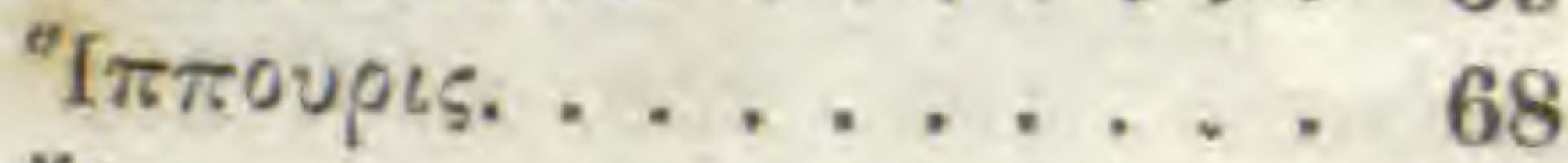

"Ipes . . . . . . . . 220

'Iøázıs . . . . . . . 48. 57

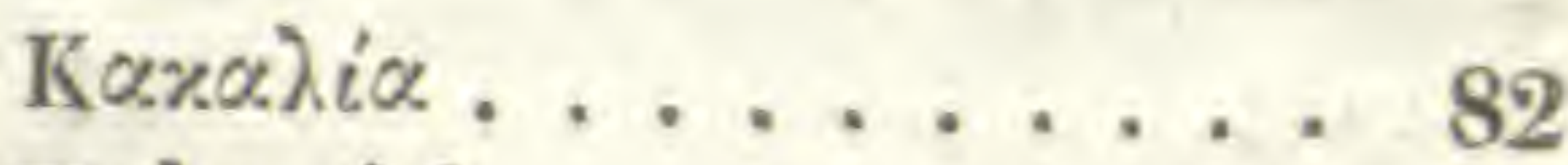

$K \alpha \lambda \alpha \mu i \nu \theta_{n} \ldots \ldots \ldots 126$

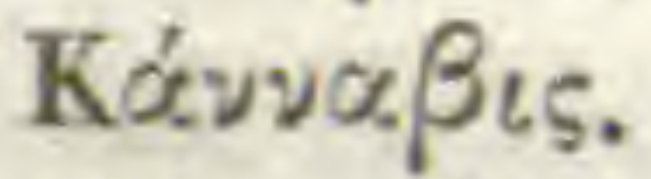

63

Kávva $\beta_{t \varsigma} \alpha \dot{\gamma} p i \alpha \ldots .242$

Kapaxia.s. . . . . . . 209

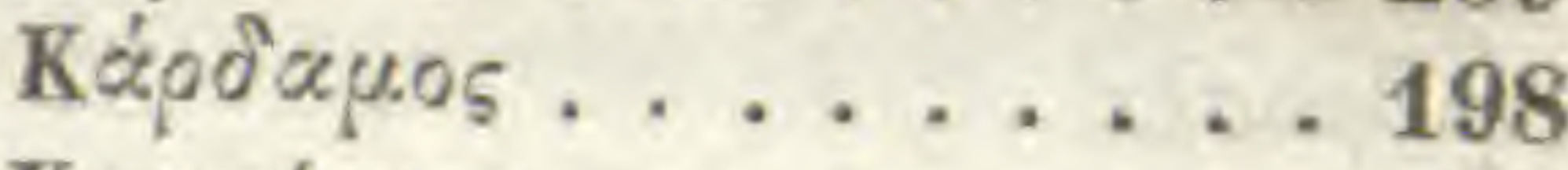

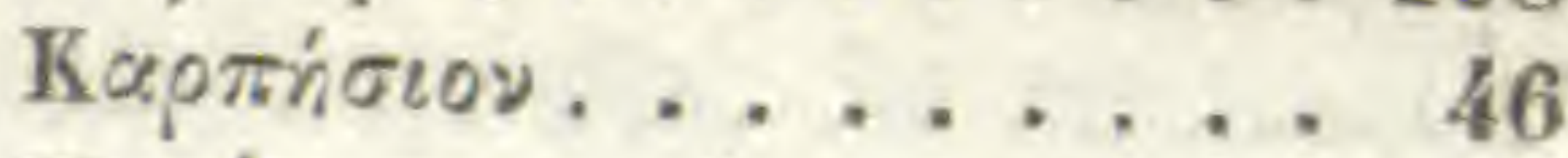

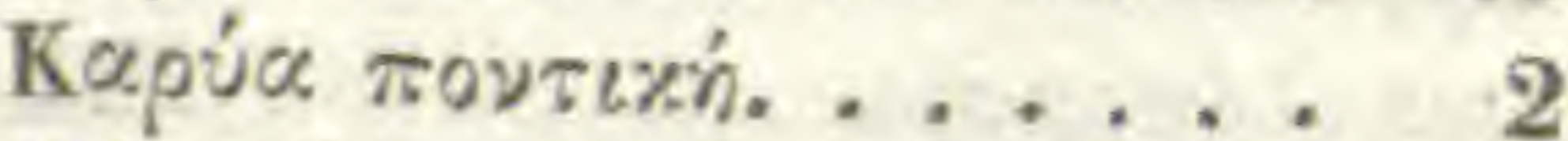

$\mathrm{K} \alpha \dot{\sigma} \sigma s u \theta \alpha \ldots \ldots . . . .130$

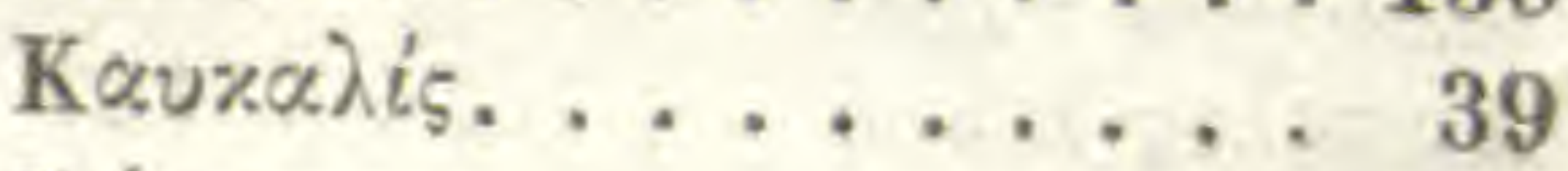

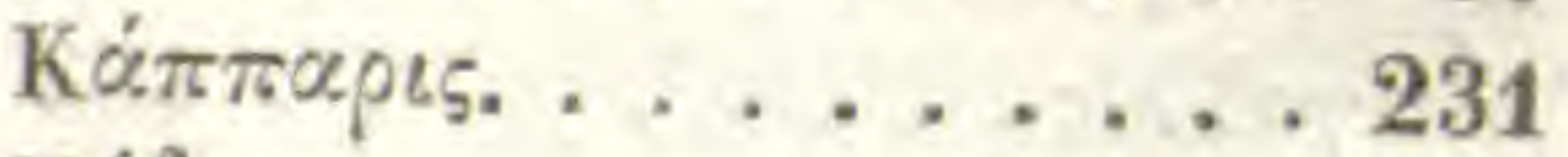

Kв́ópos........ . T

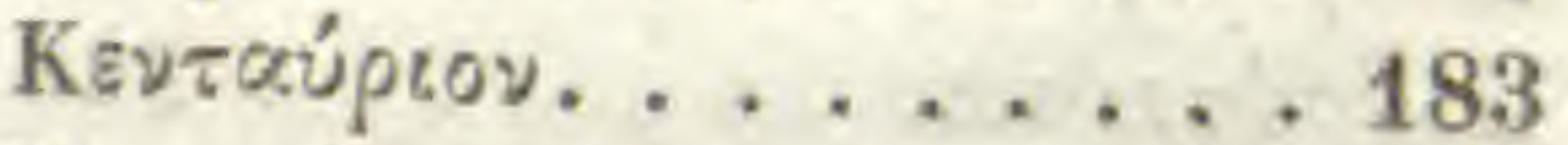

Kepwria

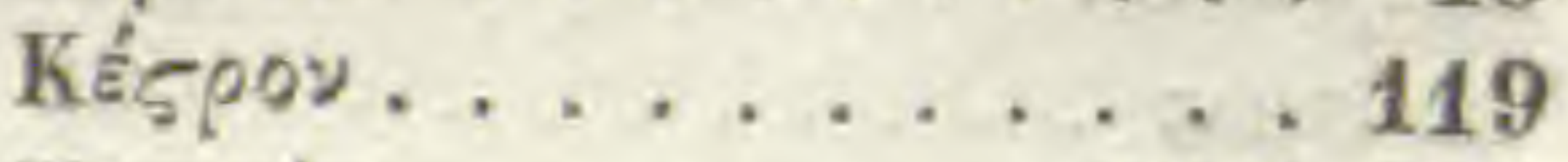

$\mathrm{K} n \pi \propto i \alpha \ldots . .261 .262$

Kízt. . . . . . . . . . 208

Kiatos . . . . . . . . 234

$K \lambda \dot{n} \theta \rho \alpha$

5

Kinparis... . . . 237. 184

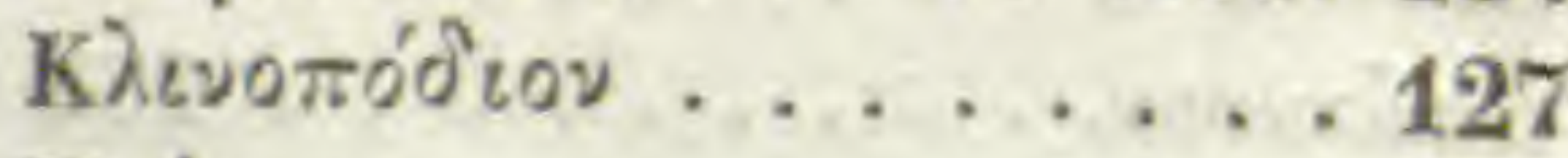

Kvé́w pov . . . . . . 234

Kvixos . . . . . . . . 98

Ko入ovté́. .......... 12

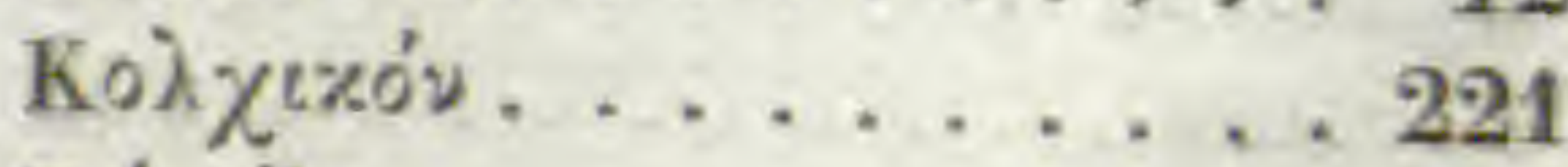

Kóvนไ̧ . . . . . . . 87

Kópes... . . . . . . 228 
Кораขо́точร ........ 157

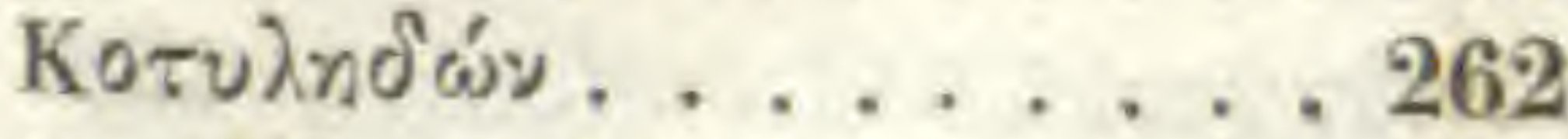

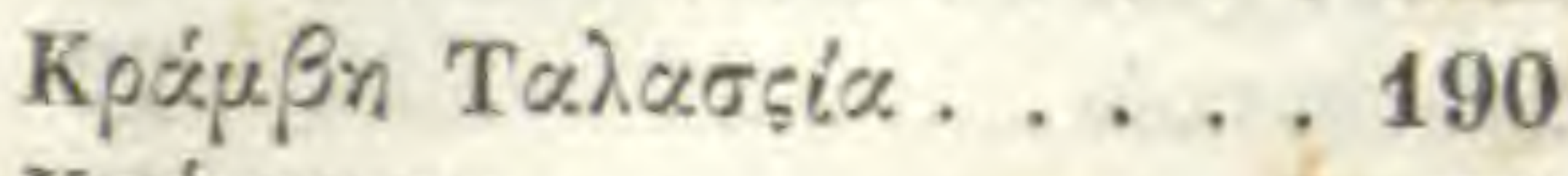

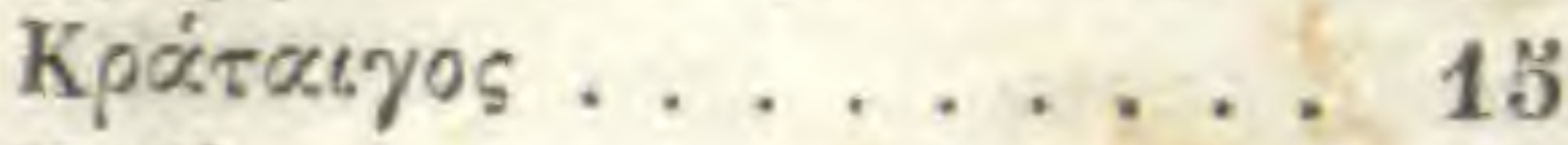

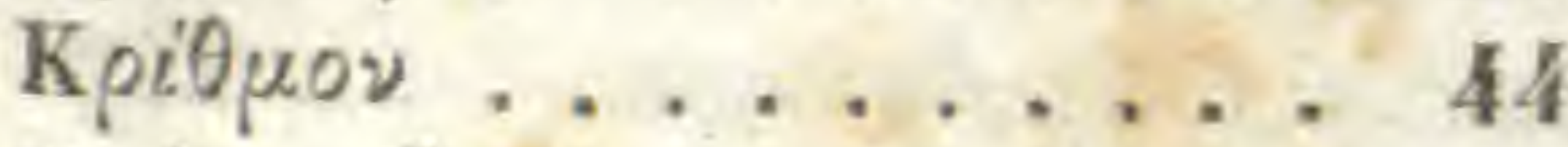

Kpivav äyptov . . . . . . 219

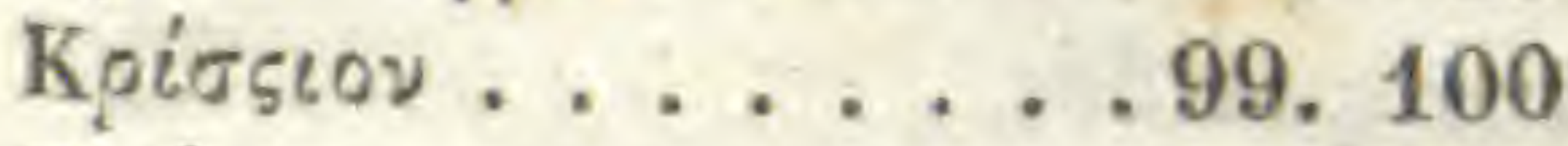

Кро'хоь ..........221

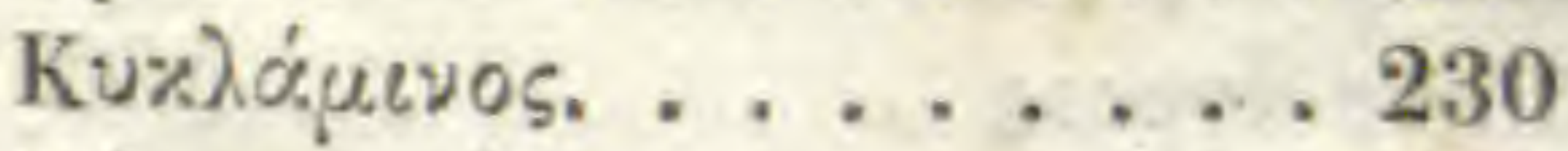

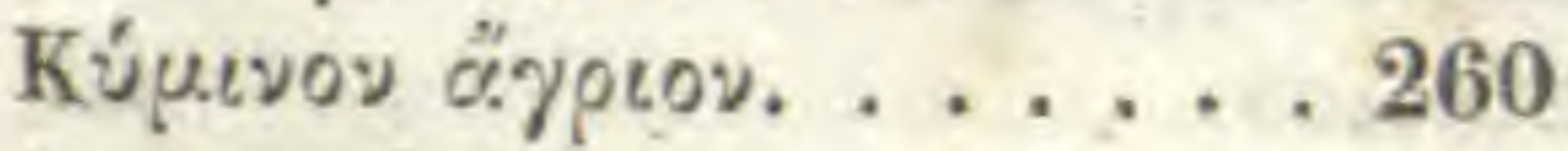

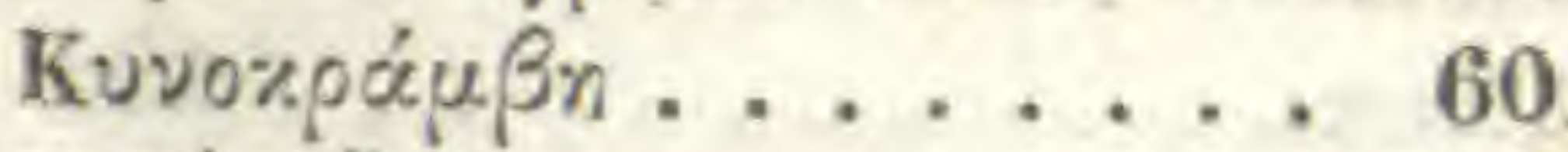

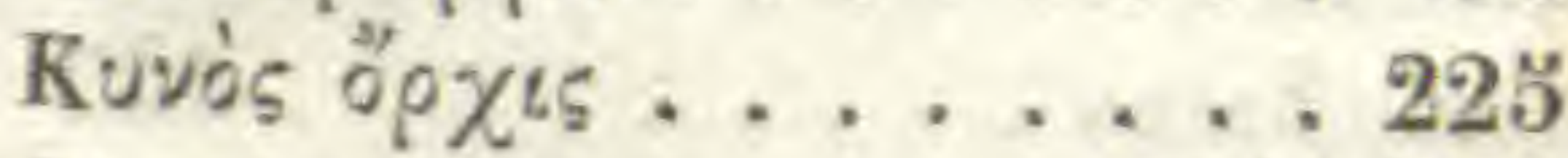

К итарьбцікц . . . . 209. 212

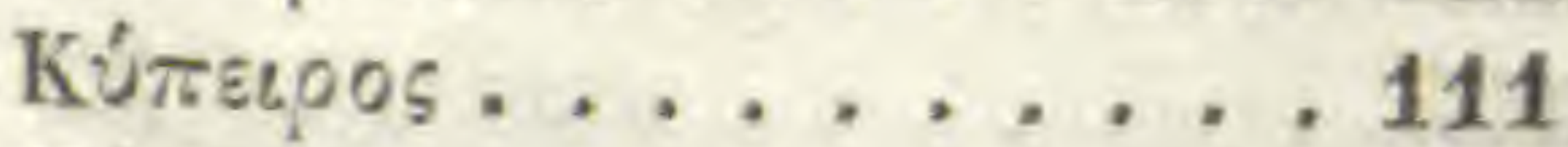

Kúmpos.......... 11

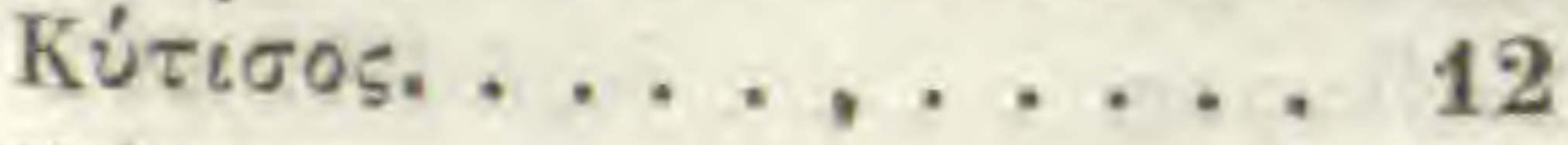

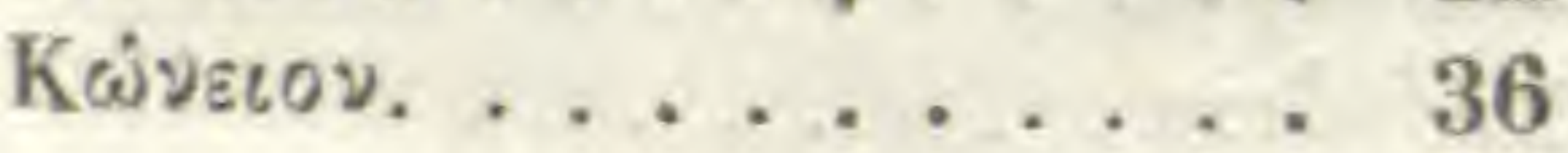

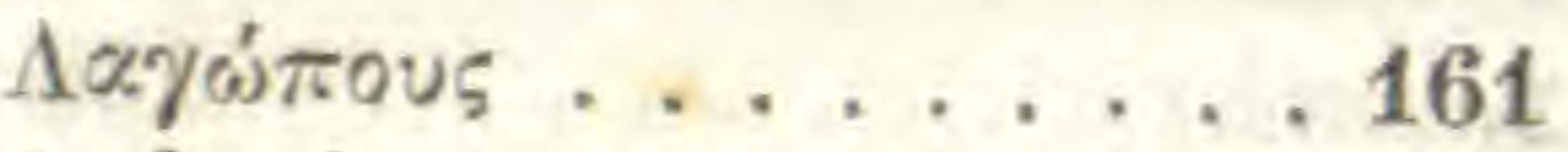

Aatupis .........212

$\Lambda \alpha \mu \psi \alpha \dot{\alpha}$. . . . . . 193

$\Lambda \alpha \dot{\alpha} \alpha \theta_{0 \nu} \ldots \ldots \ldots . .59$

$\Lambda \varepsilon \iota \chi^{\dot{n} \nu} \ldots \ldots . . .65$

$\Lambda \varepsilon \pi i d^{2}\llcorner 0 \nu . . . . . . .198$

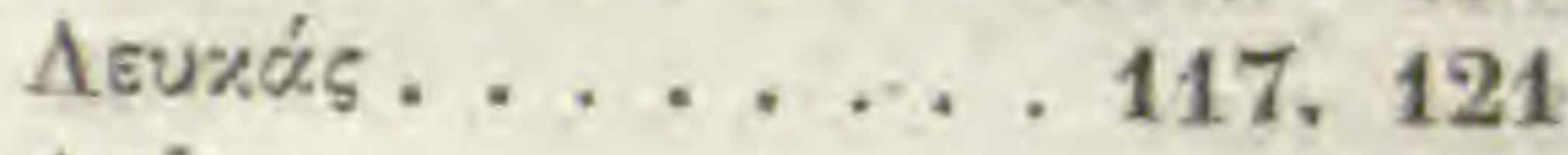

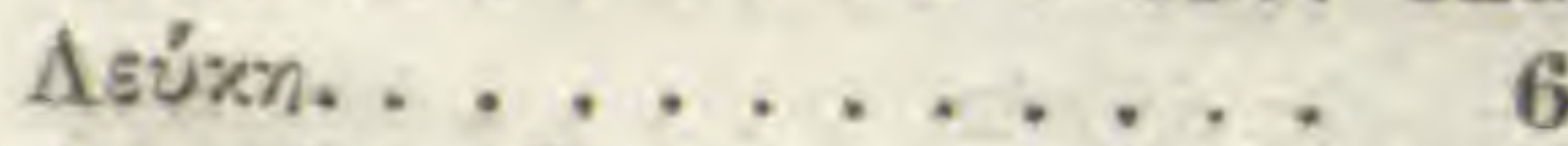

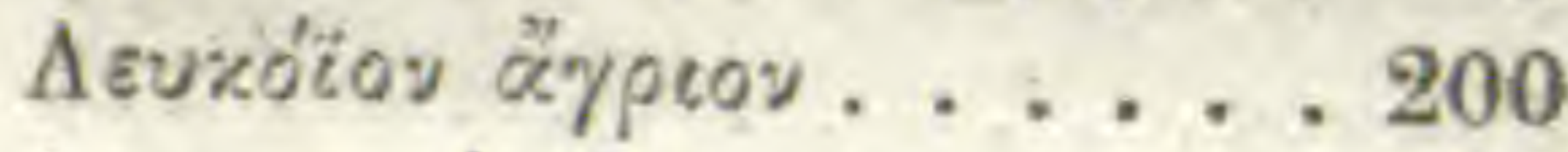

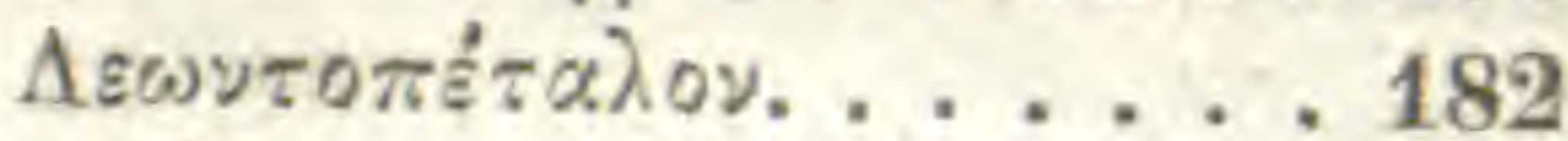

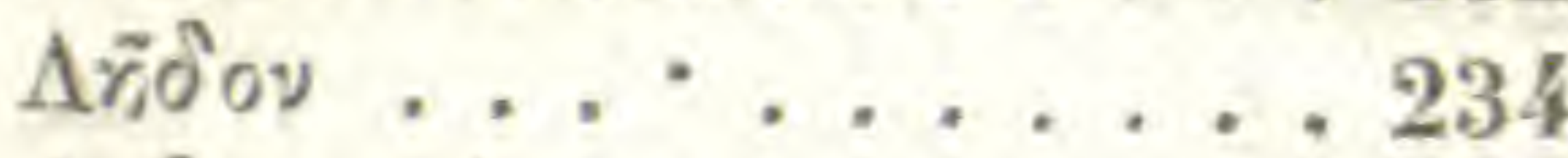

$\Lambda \iota \beta \alpha \nu \omega \tau i \zeta \ldots . .22 .72$

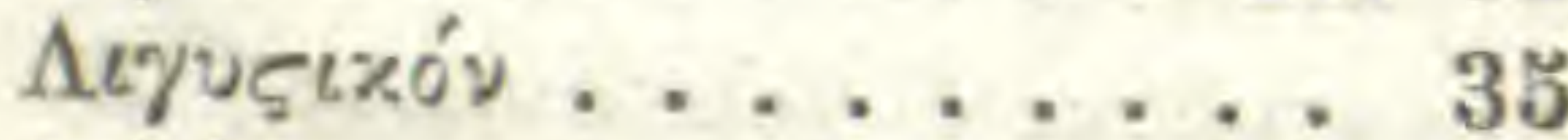

$\Delta \imath \theta \dot{\sigma} \sigma \rho \varepsilon \rho \mu_{0}, \ldots \ldots . . .54$

Atvó́çรเs ......... 192

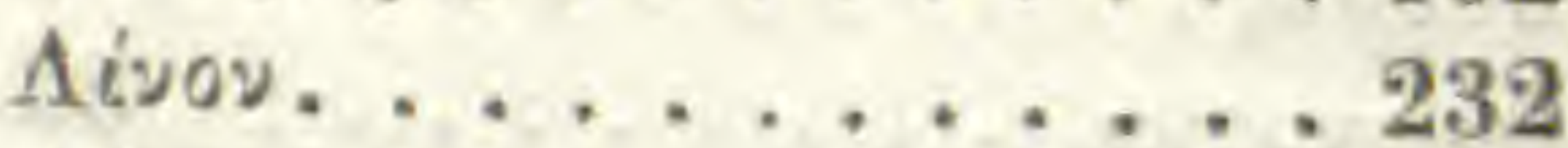

Ai $\chi^{v e s} \ldots . . .1151 .152$

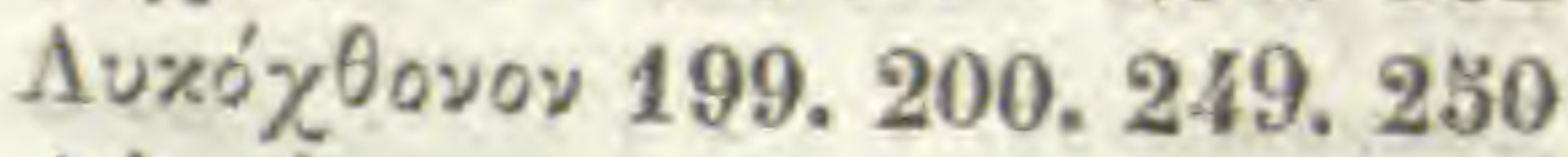

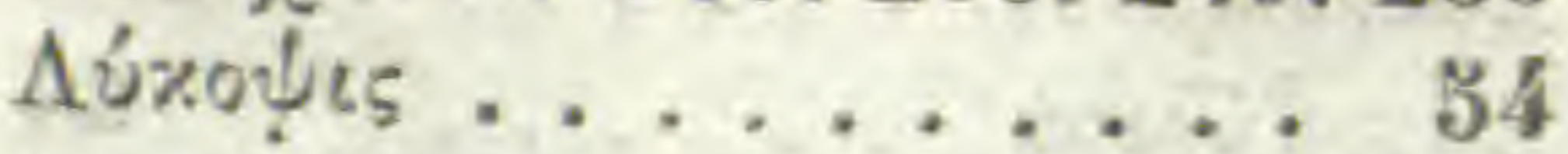

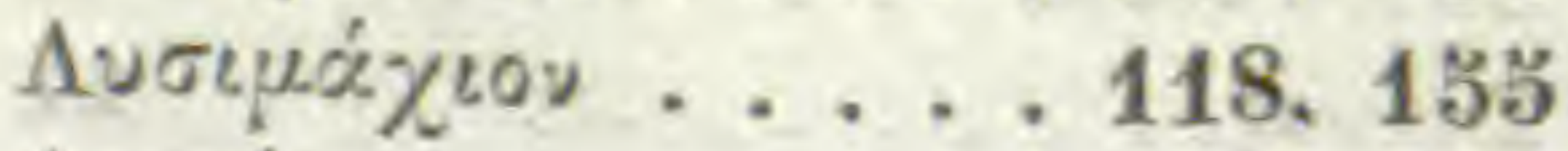
Awrós ........ 19. 20

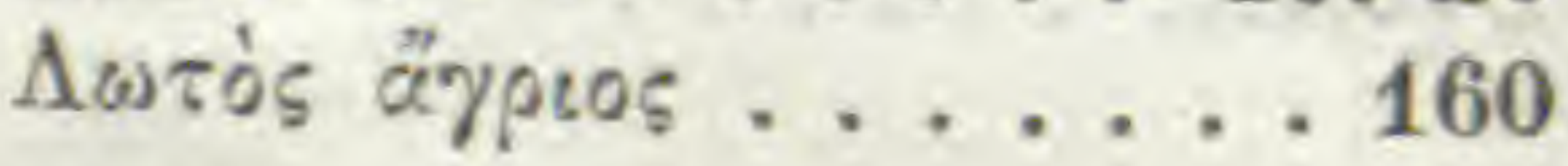

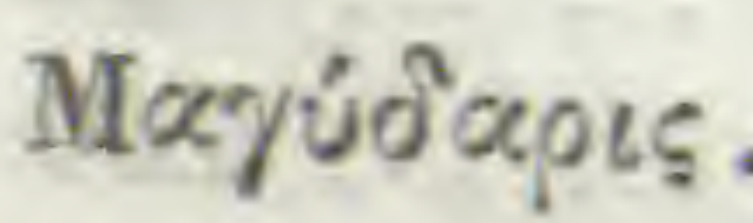

$M \alpha \lambda \alpha_{\alpha}^{\prime} \chi^{n} \ldots \ldots . . .244$

Mavóp'́ryopas. ....... 148

Mápov .......... 128

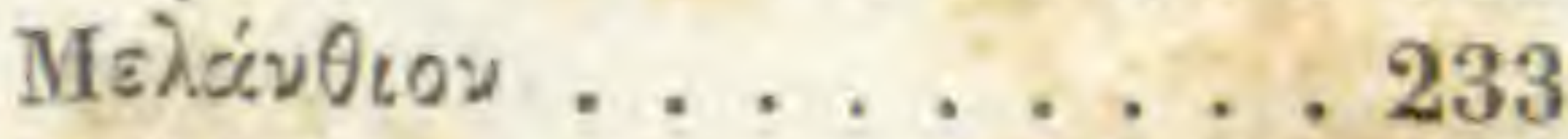

$M \varepsilon \lambda i \alpha^{\prime}, \ldots \ldots \ldots, 3$

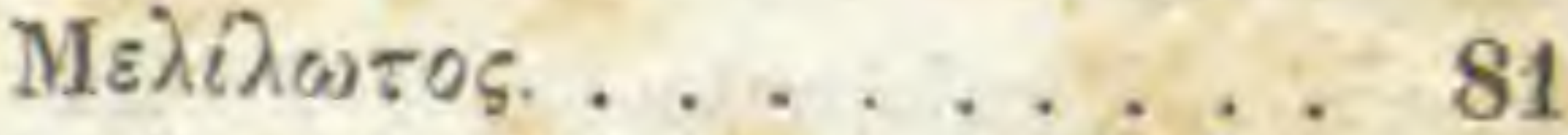

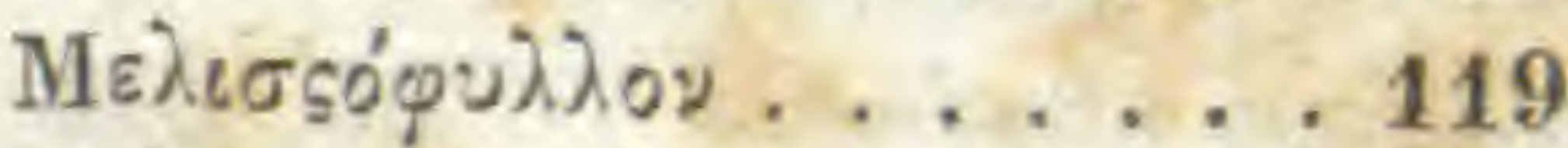

Mย́์ข. . . . . . . . . 21

Mnd $2 x$ मे. . . . . . 160. 161

Mńdเov.... . . . 235. 236

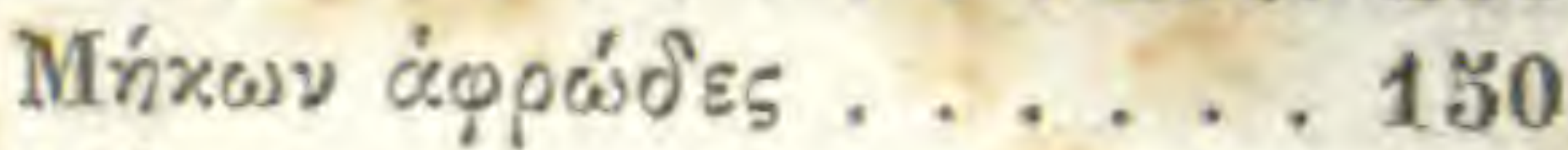

Mи́n

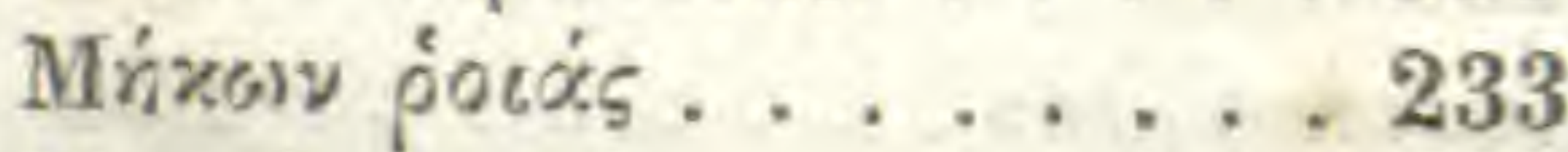

Mupsixn.

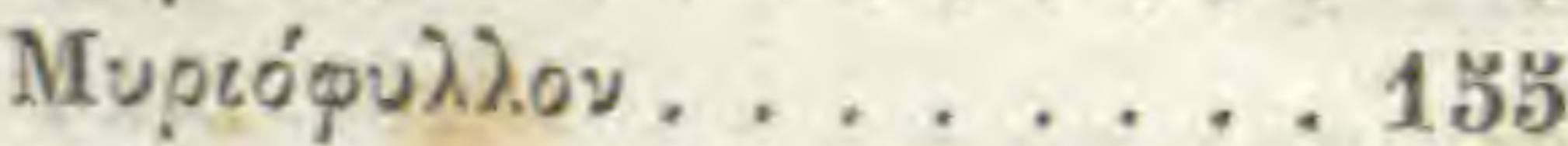

Múppes........... 37

Mupaivn........... 16

Mupritns. . . . . 209. 210

Mát. . . . . . . . . 224

Nápdos ópstขń ...... 45

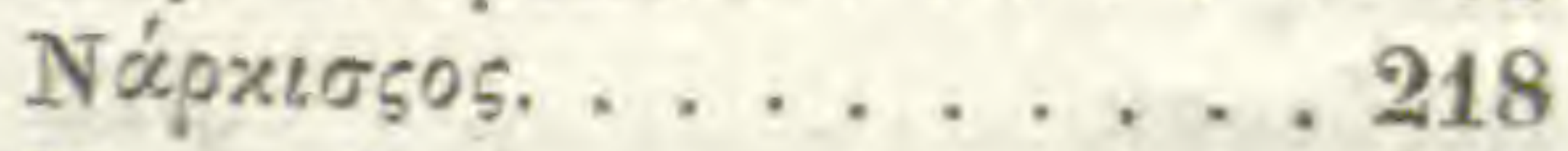

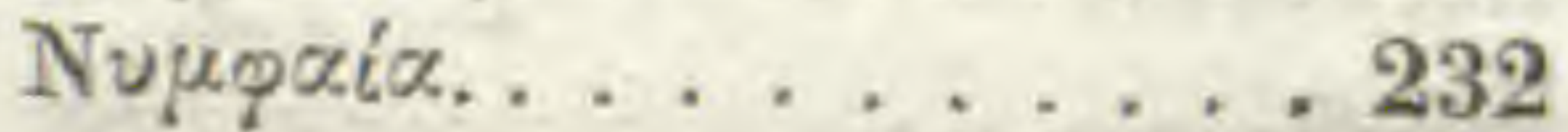

$\Xi \dot{\alpha} v \theta$ เov. . . . . . 207

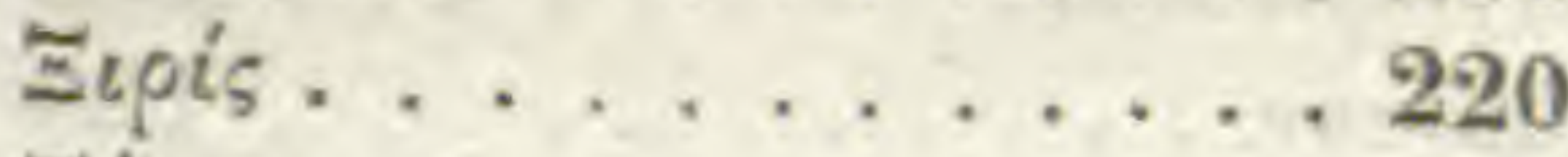

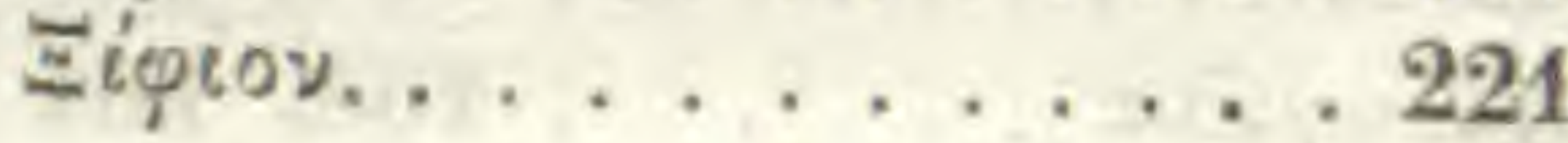

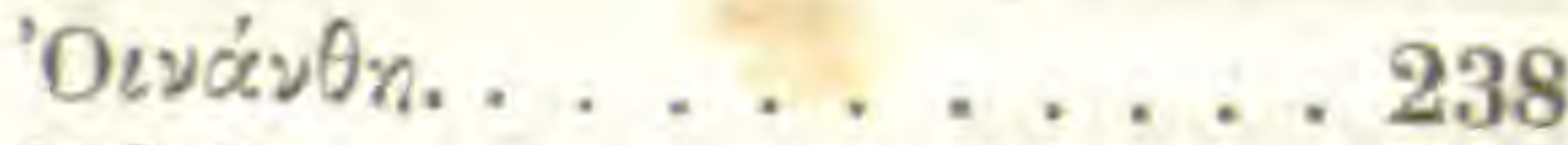

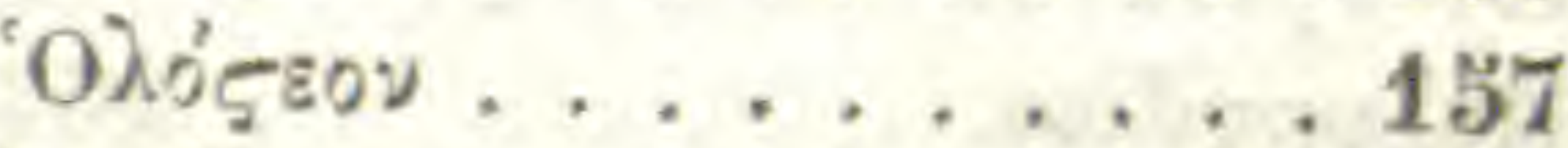

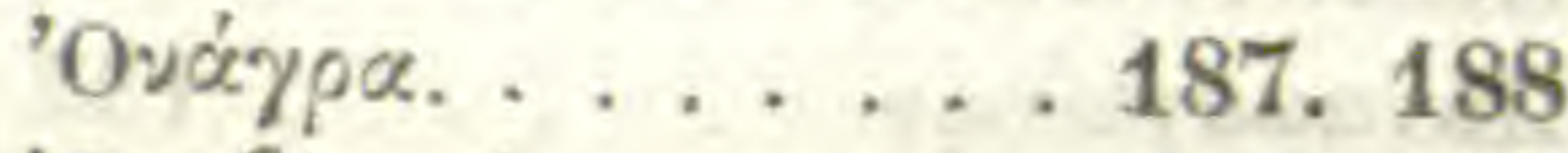

'Oroßpuxis. . . . . . 165

'O $\dot{\alpha}^{\alpha} \lambda_{\iota 5} \ldots . . . . . . .59$

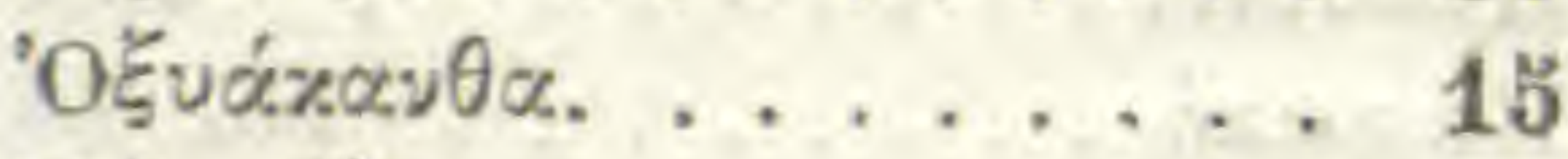

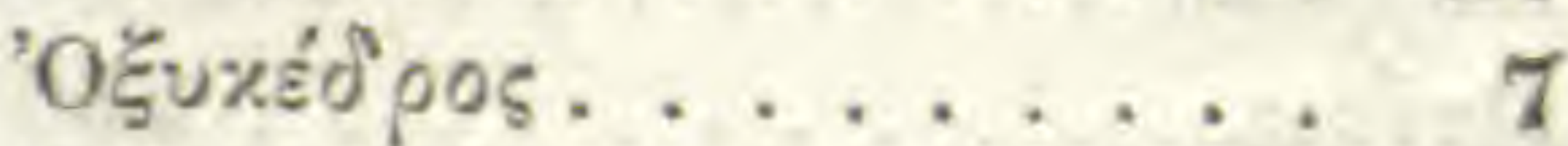

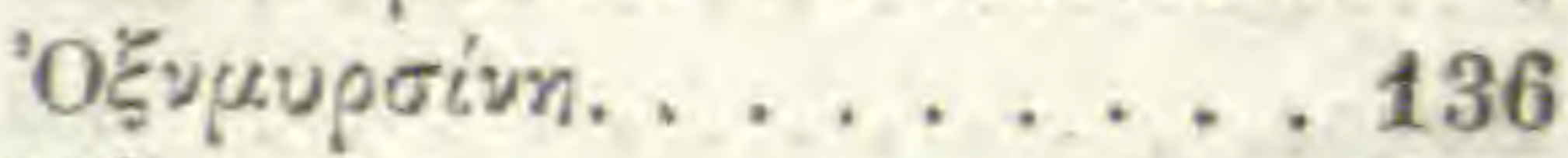

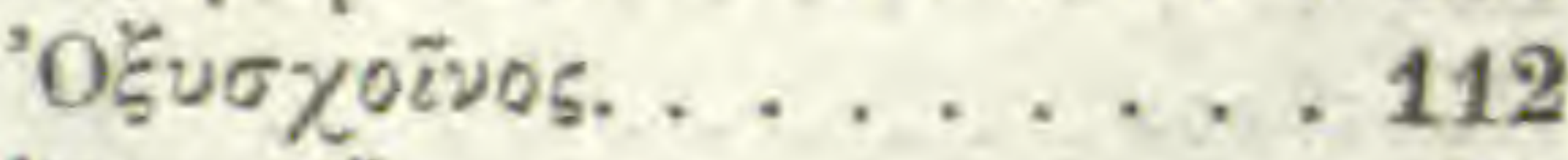

'OดE०ศÉ

'Optrovos. . . . . . . 128

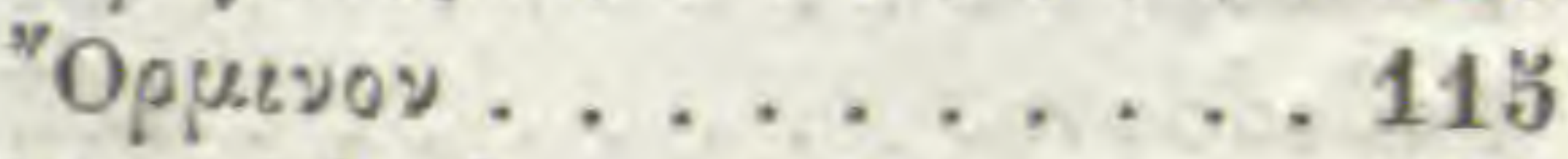

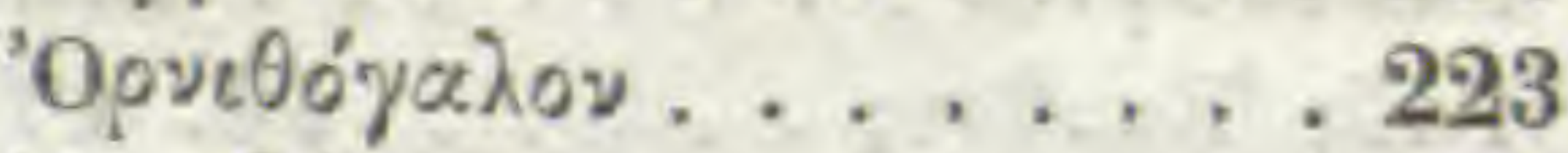

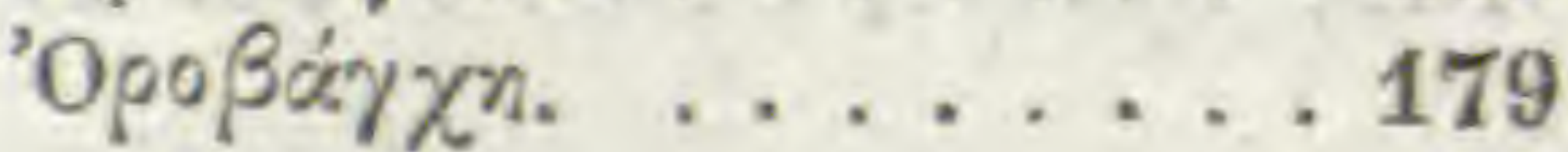


"Oquors........... 175

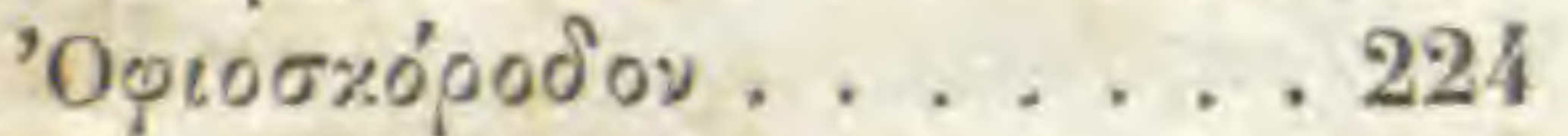

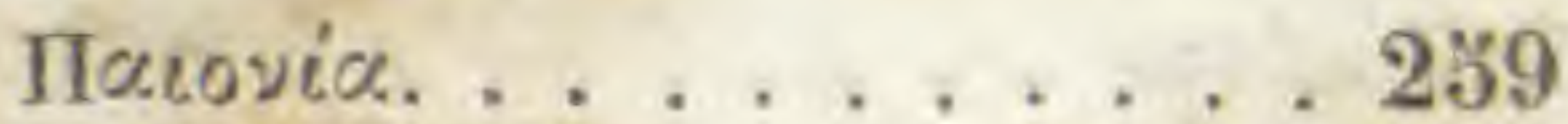

$\Pi \alpha \lambda$ เoúpos. . . . . . . . . 15

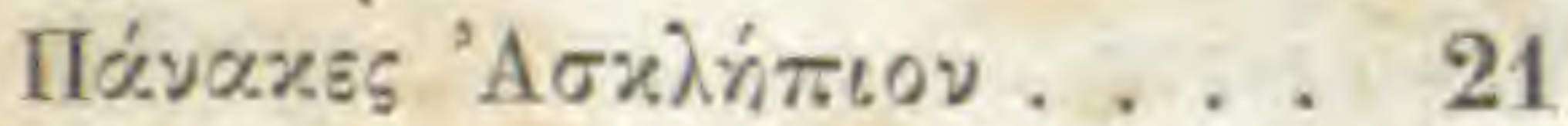

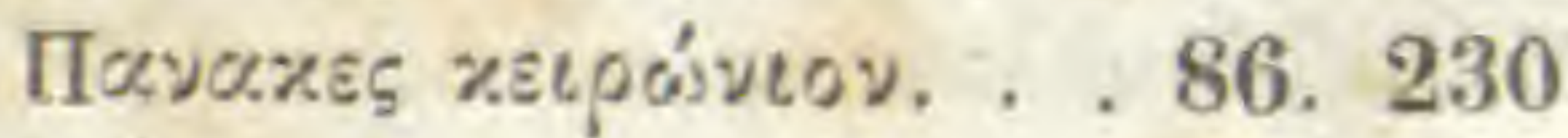

Пйтuроs . . . . . . . . 110

Пкри่́

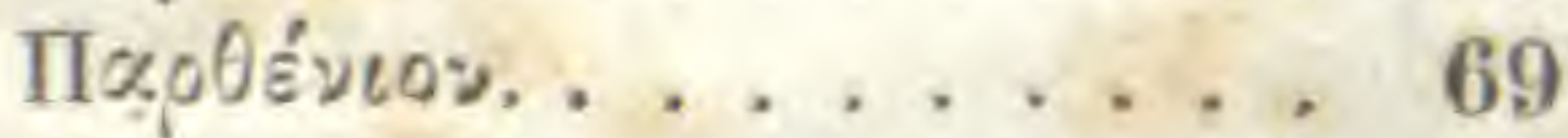

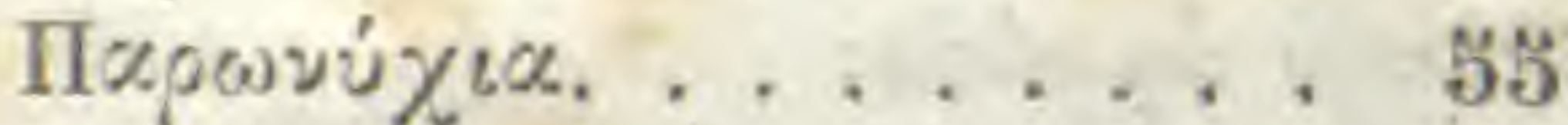

Пв

$\Pi \varepsilon \nu \tau \dot{\alpha} \varphi \gamma \lambda \lambda_{0 \nu} \ldots . . .2 .239$

$\Pi \varepsilon \pi \lambda_{\mathrm{s}} \ldots . . .2 . .213$

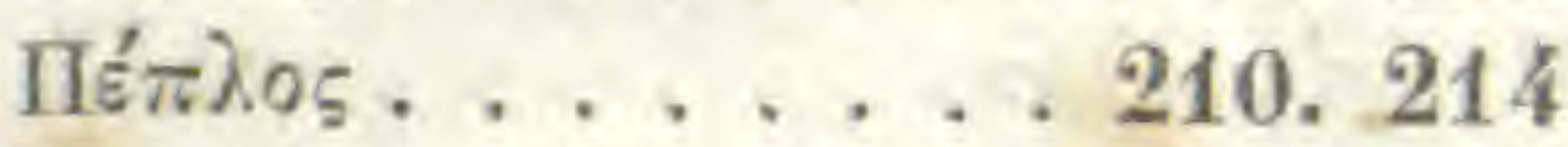

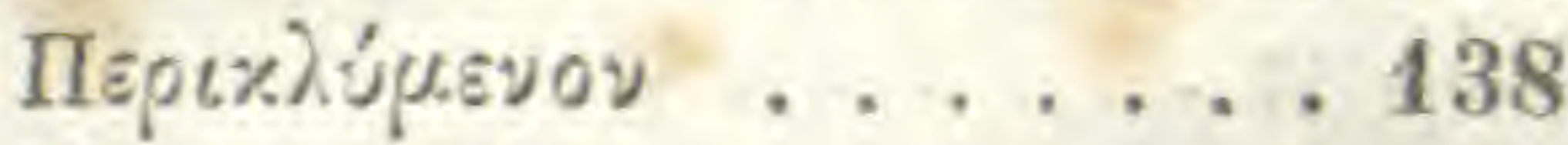

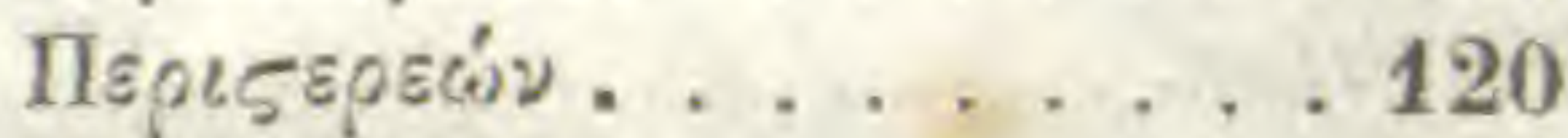

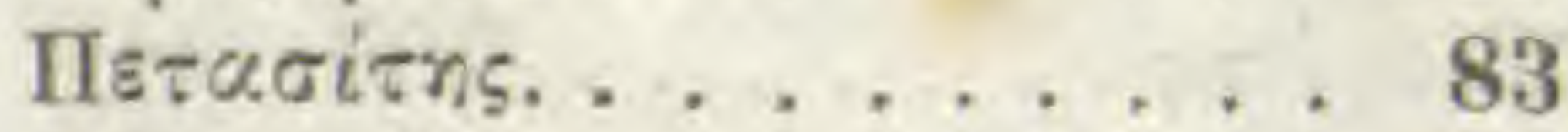

Пвтробе่́

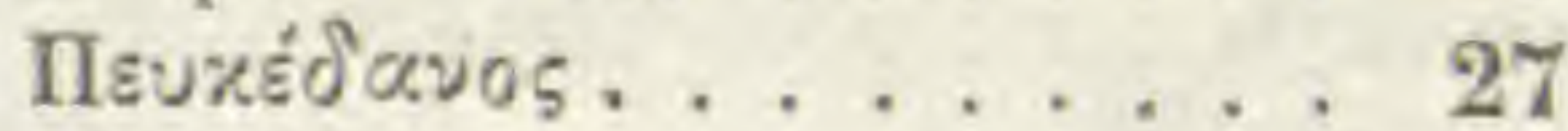

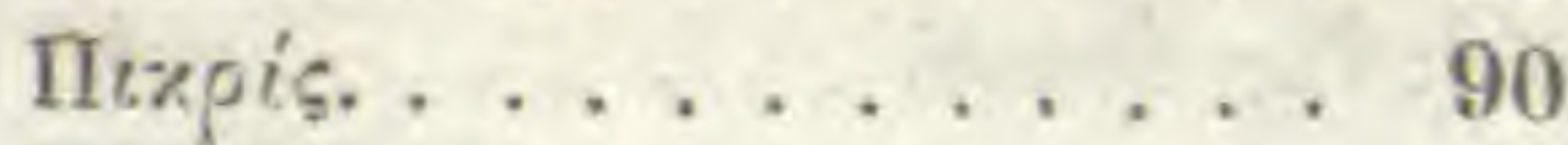

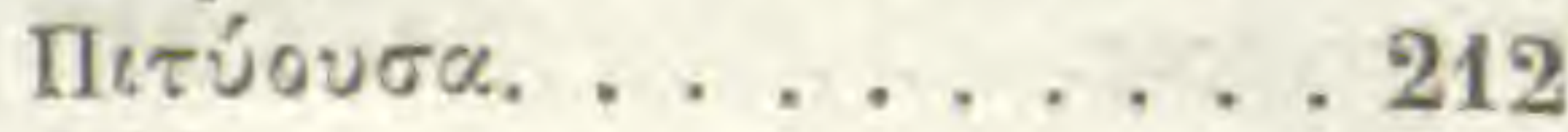

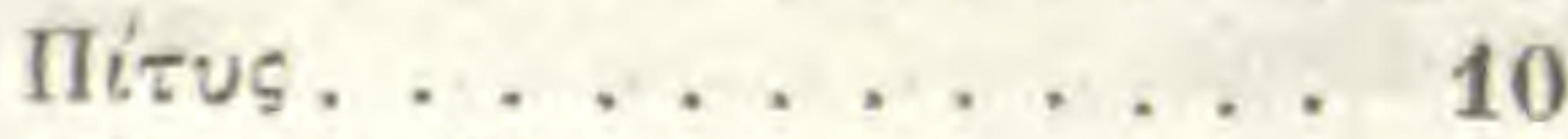

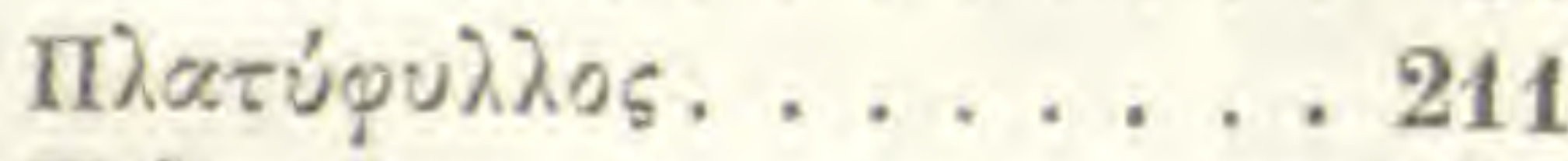

Пофะนผ่ขเoข. . . . . . . . 152

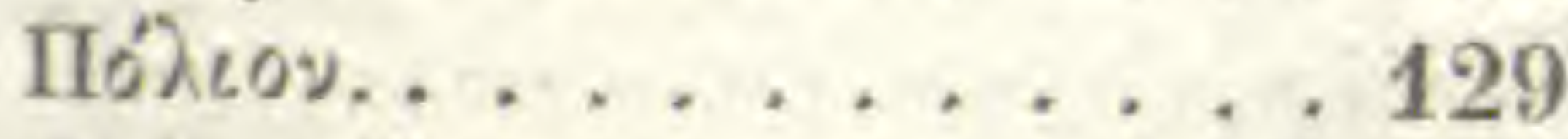

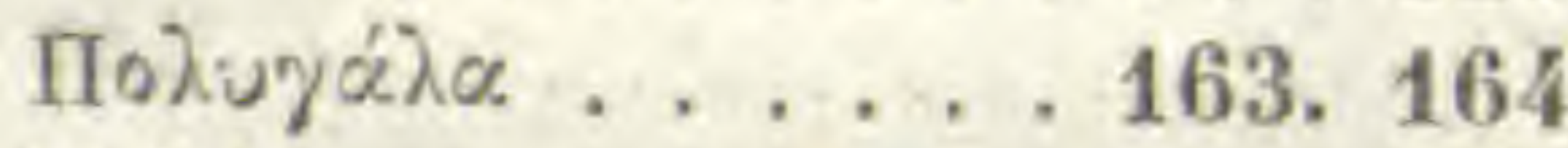

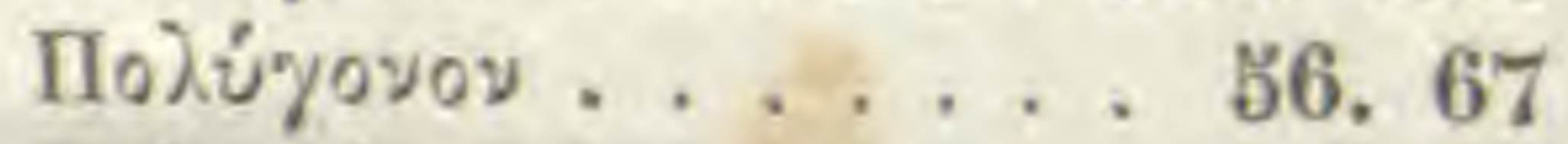

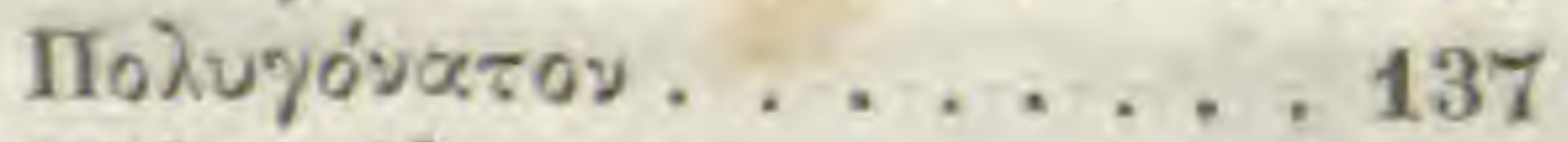

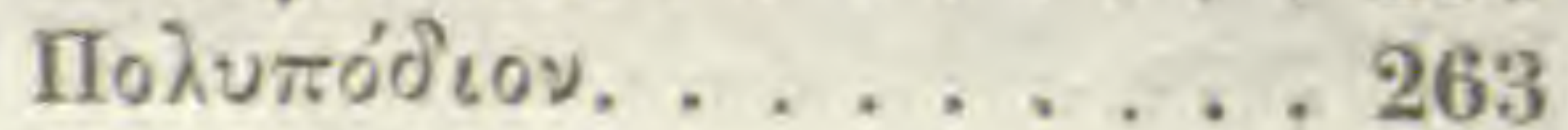

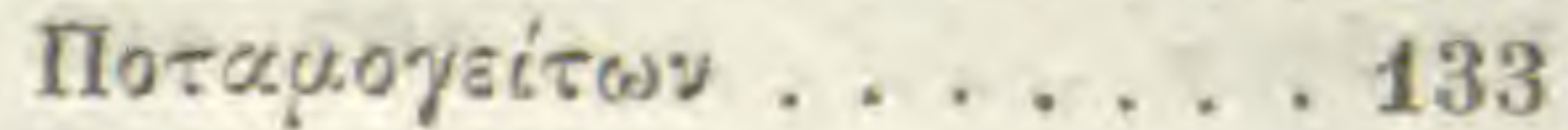

Пorńpıov . . . . . . . 138

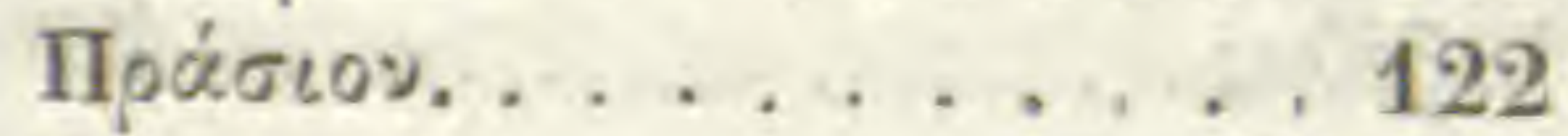

Пхррихй ... . . 73, 74

Пте́рt६. . . . . . . . 266

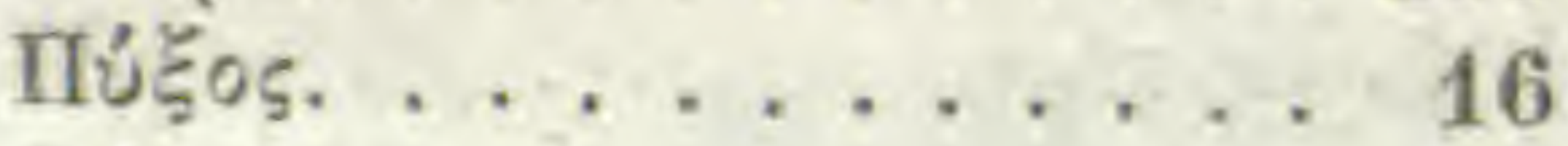

Пúpe日pov......... 40

'Pяp.vos. .......... 14

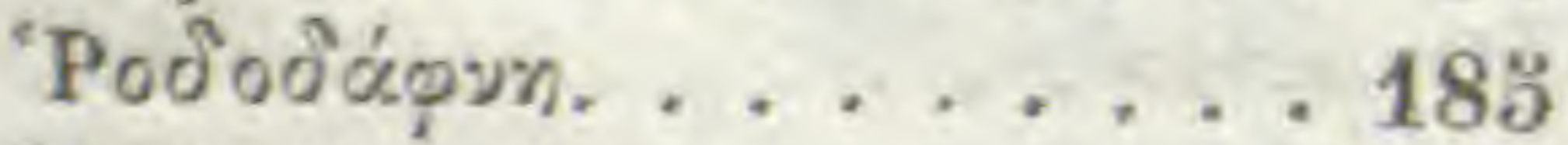

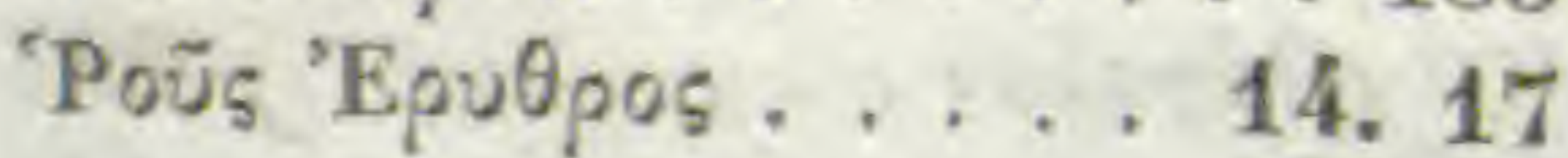

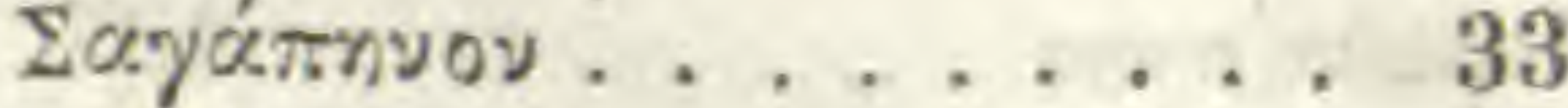

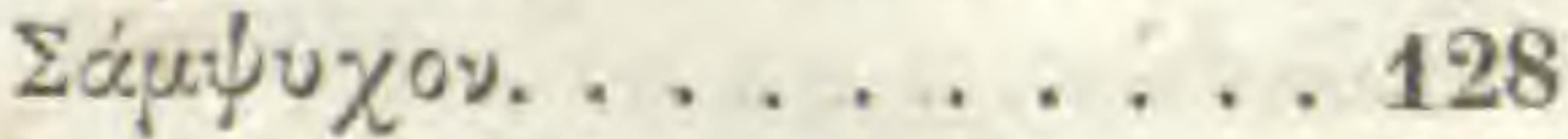

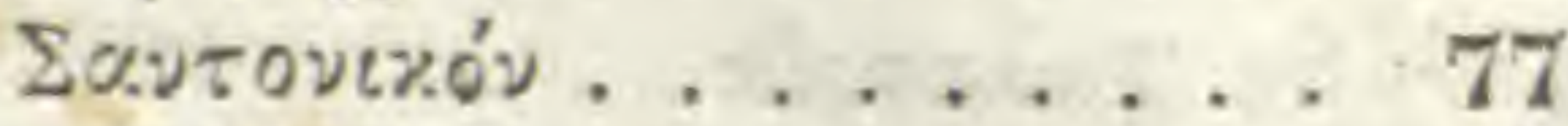

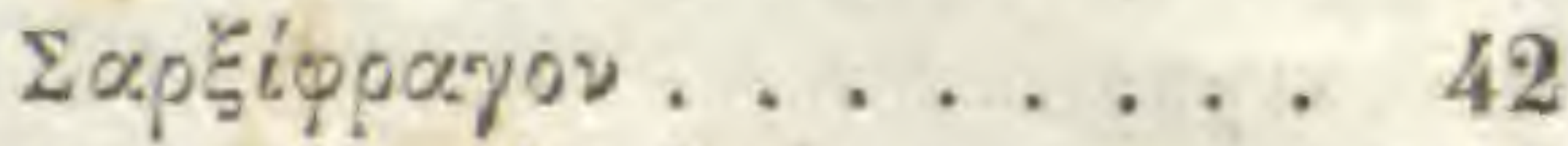

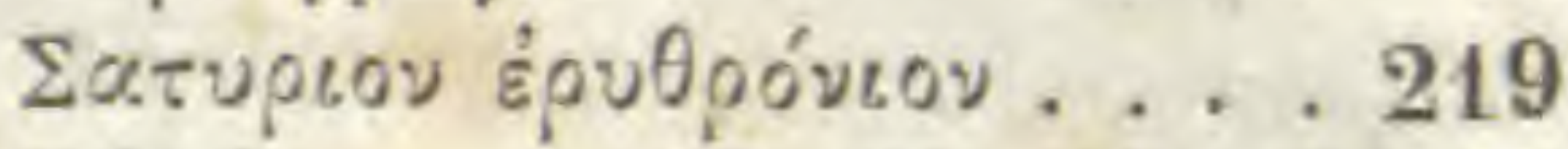

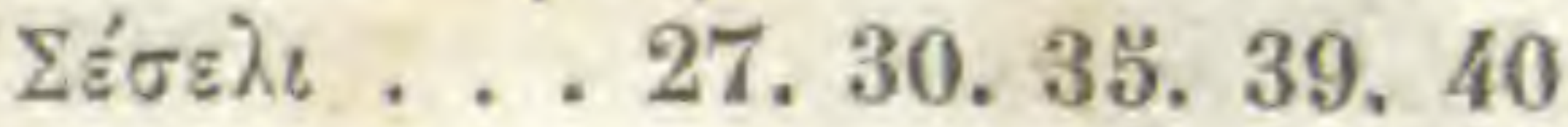

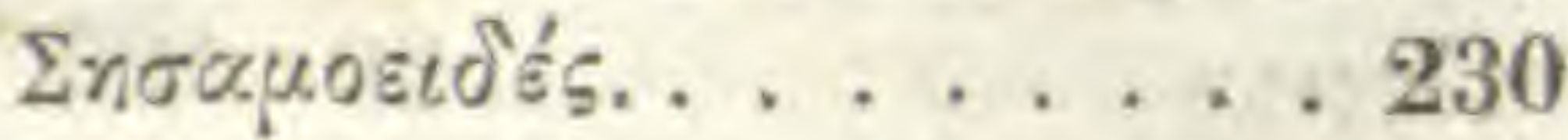

$\Sigma \iota \delta ’$ рітє૬. 124, 125. 172, 257

sixus äyotos . . . . . . 206

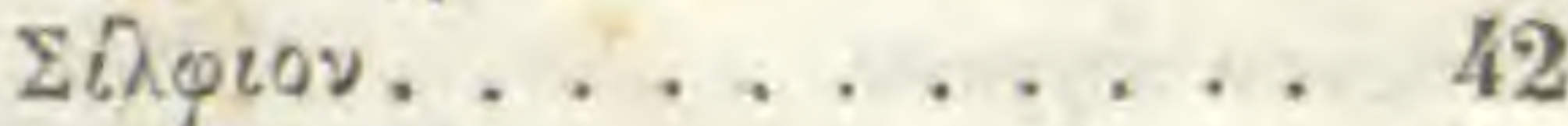

$\Sigma i v n \pi t . \ldots \ldots \ldots . . . .194$

$\Sigma_{\iota \sigma i \mu \beta \rho\llcorner o \nu} \ldots . . .1 .127$

$\Sigma i \sigma \omega \nu . . \ldots \ldots \ldots .40$

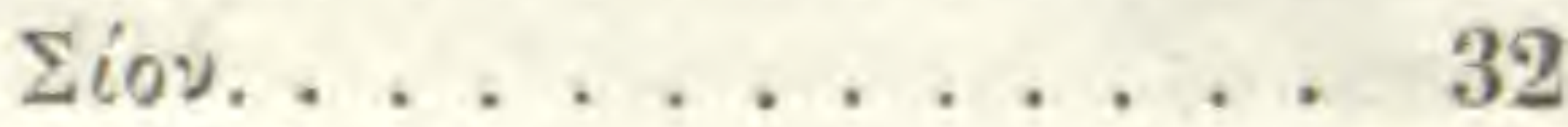

$\Sigma_{x \alpha \nu d^{2} \iota \xi . . . . .2 .257}$

$\Sigma \times 0^{\prime}$ นu. . .....99. 101

$\Sigma x 0^{\prime} d^{2} l o v \ldots . . . .130$

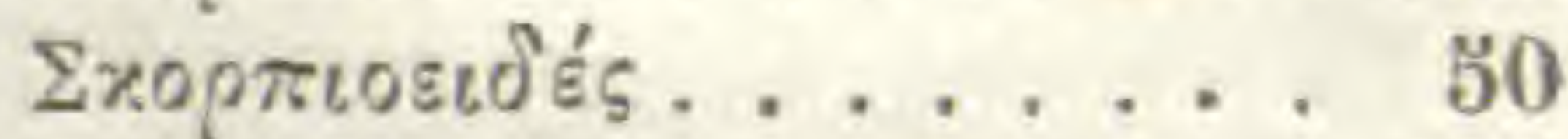

¿zootios ........... 158

$\Sigma \mu i \lambda a \xi \ldots \ldots \ldots 10$

$\Sigma u i \lambda \alpha \xi \lambda \varepsilon i \alpha \ldots \ldots . . .189$

$\Sigma u i \lambda \alpha \xi \tau \rho \alpha \chi \varepsilon i \alpha \ldots \ldots .138$

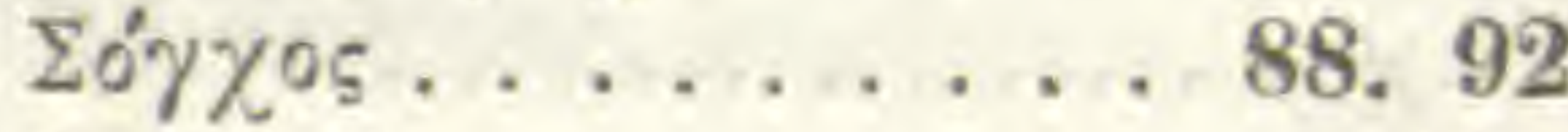

$\Sigma \pi \alpha \rho \gamma \dot{\alpha} \nu\llcorner 0 \nu . \ldots \ldots . .133$

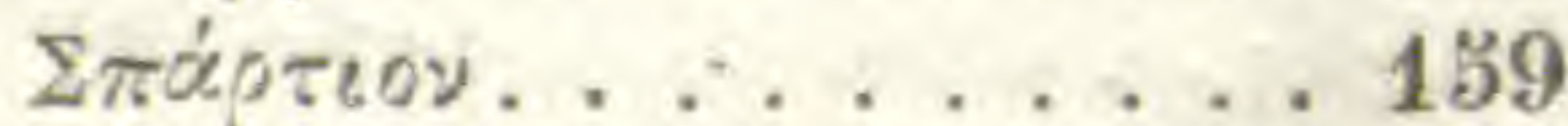

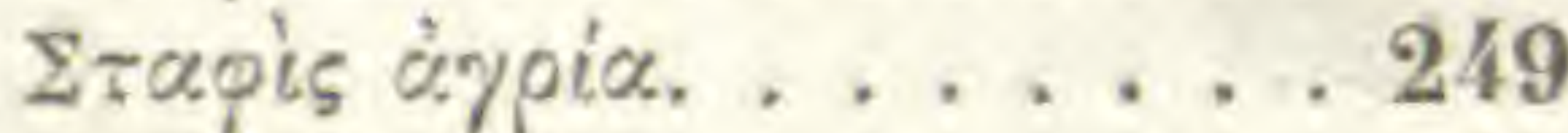

$\Sigma \tau \dot{\alpha} \chi \cup \varsigma \ldots \ldots \ldots \ldots . . .115$

$\Sigma \tau \alpha$ ou

$\Sigma \tau 0 \iota \chi \alpha \dot{\alpha} . \ldots \ldots \ldots 130$

$\Sigma \tau \rho \alpha \tau \iota \dot{\tau n s . ~ . . . . . . . ~} 263$

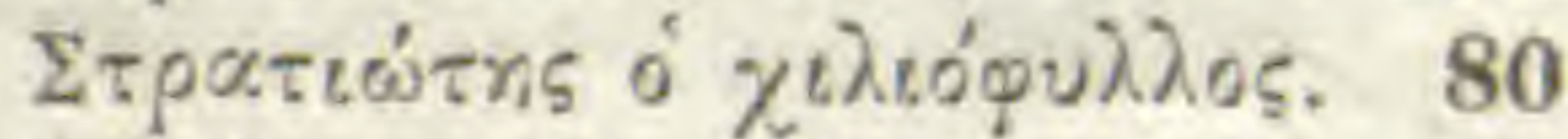

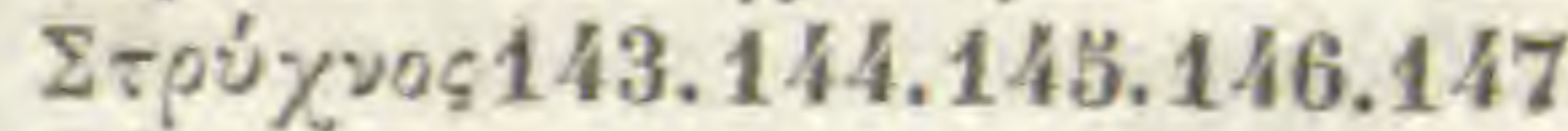

$\Sigma \tau \dot{\rho} \rho \alpha \xi \ldots \ldots \ldots . . .1$

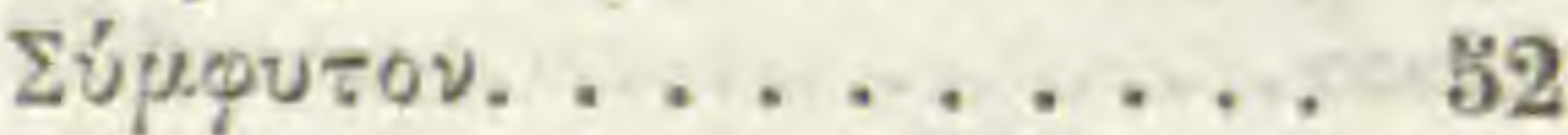

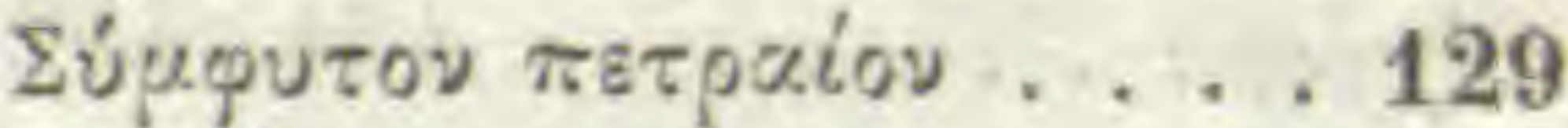

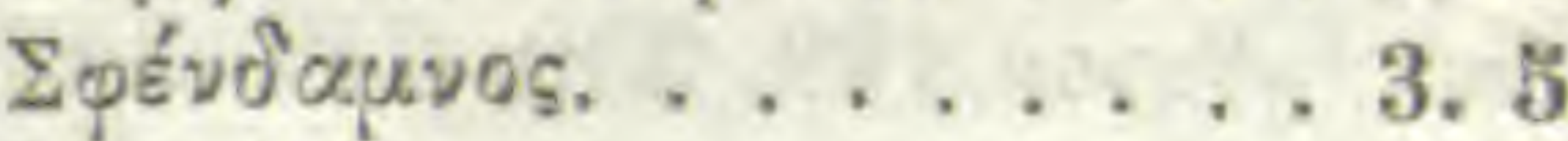

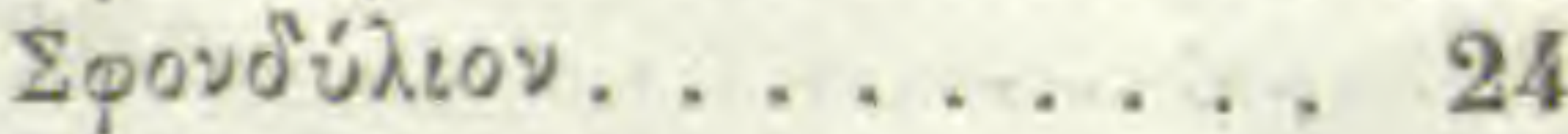

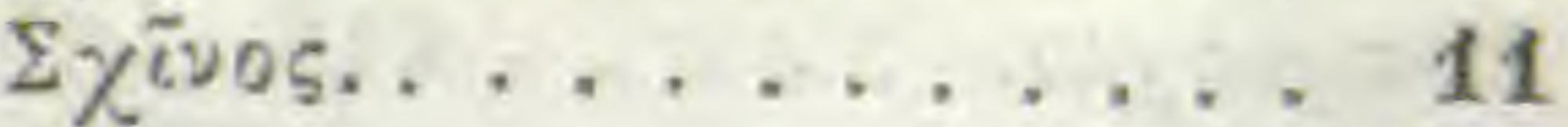

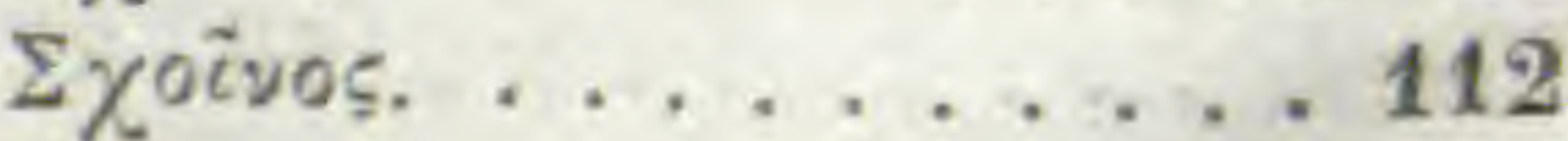

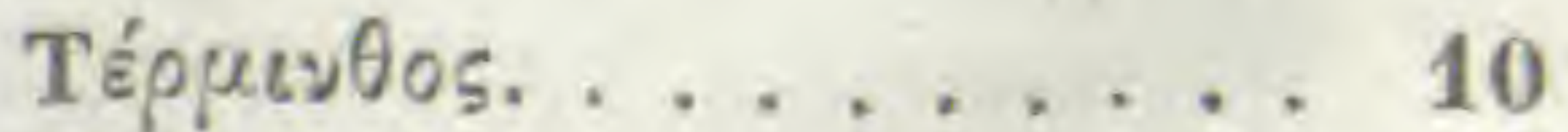

Tészptov. .......... 130 
Тท่́าเs. . . . . . . 1899

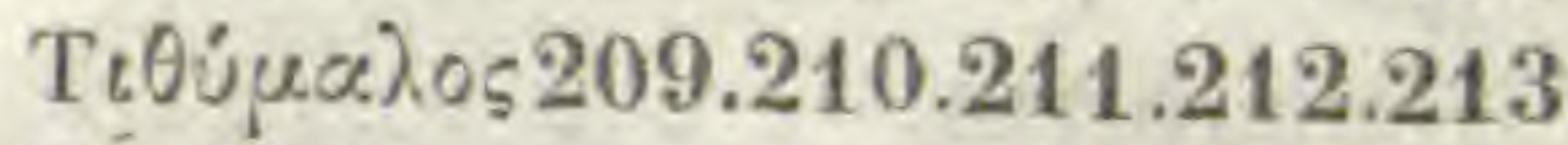
Topớricos ...... 32. 40 Tрауорíavos...... 121. 125

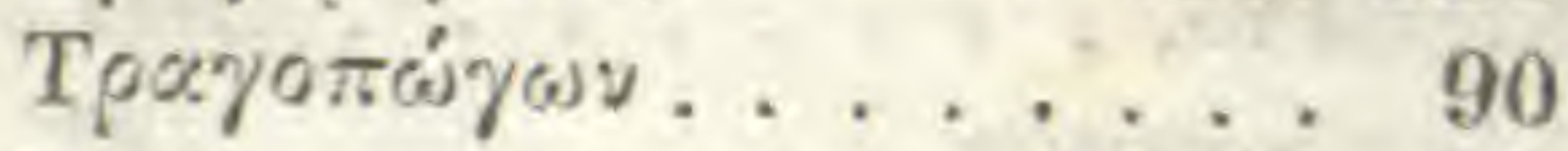

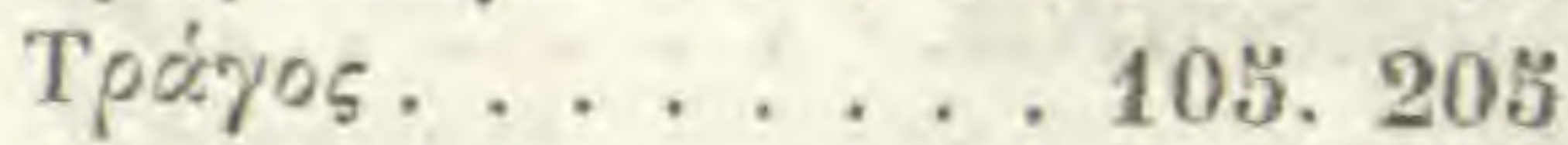
Tpißolos ........165

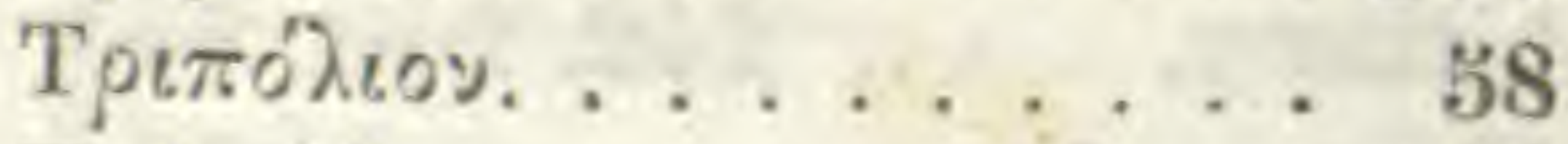

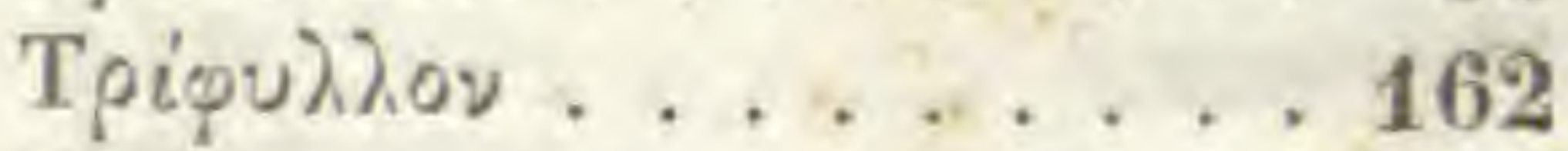

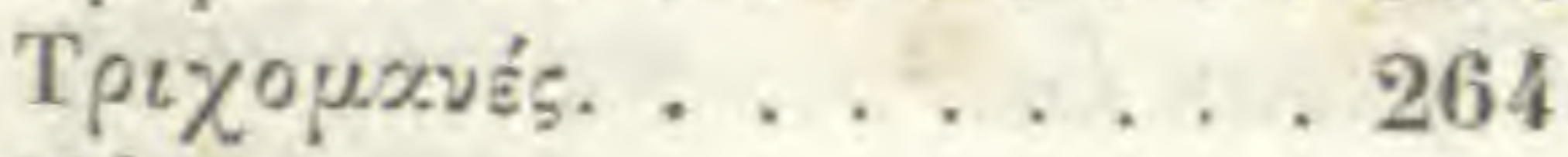
Tự̆ ..........112

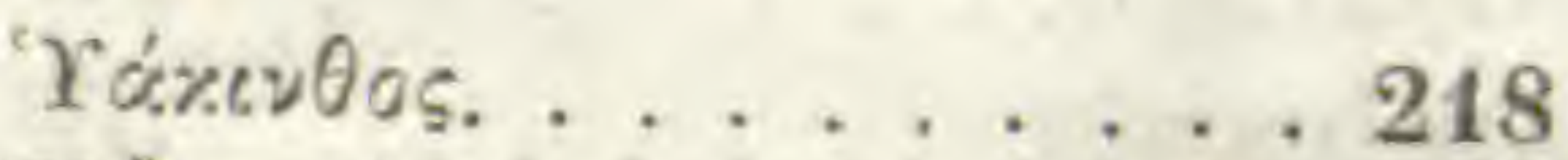

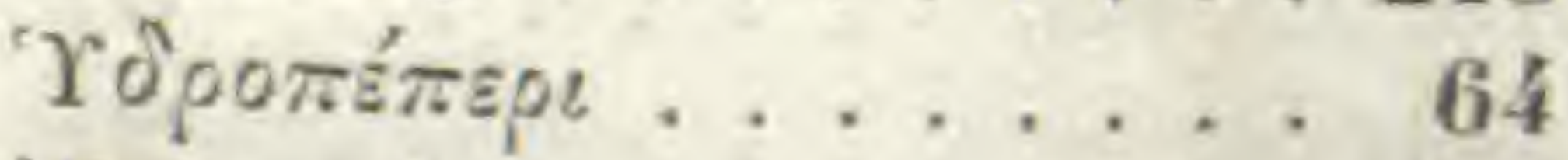

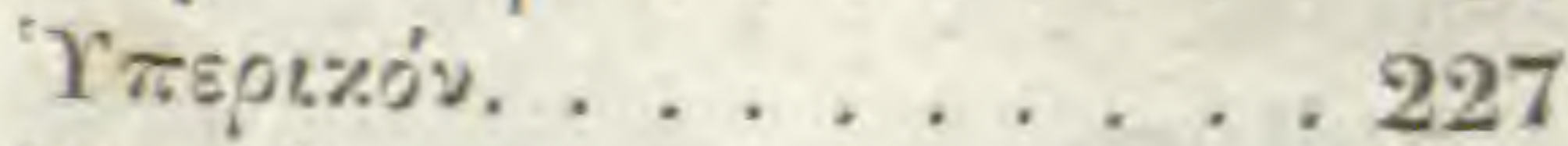

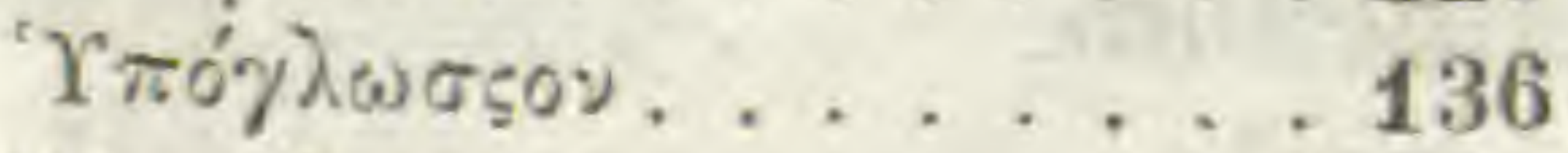

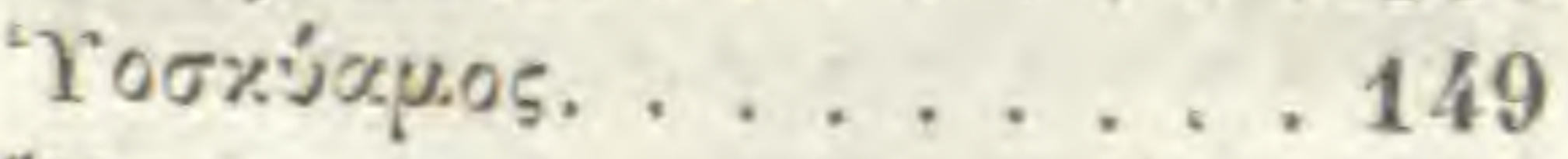

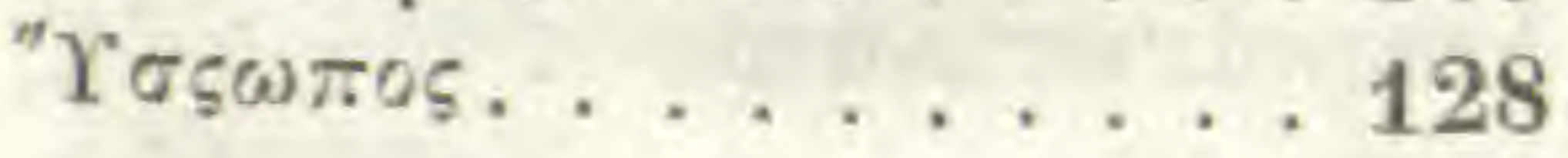

$\Phi \alpha \lambda \dot{\alpha} \gamma \gamma(o \nu \ldots . .2 .216$ $\Phi \alpha^{2} \lambda \lambda$ apes ......... 107

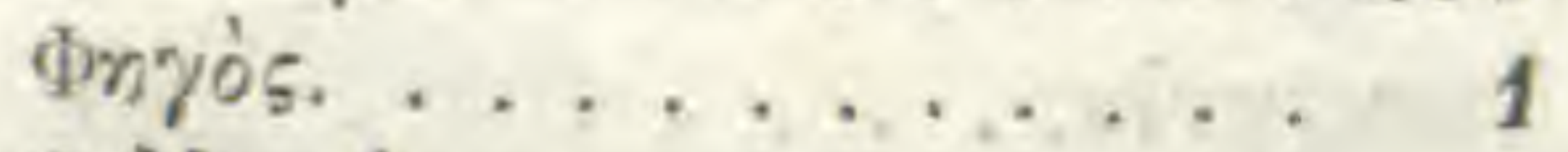

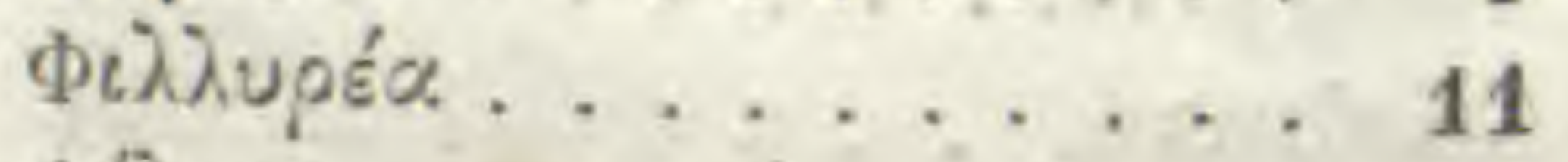
$\Phi$ ìupa.......... 2

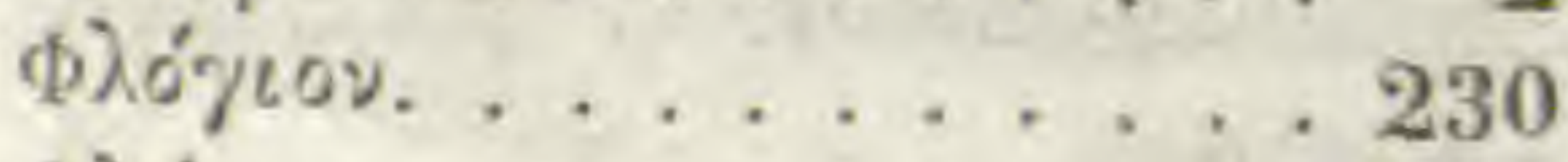
Фोóp..5. . . . . . . . . 149

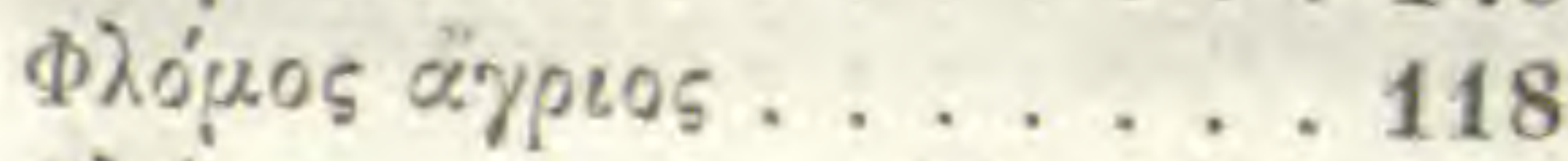

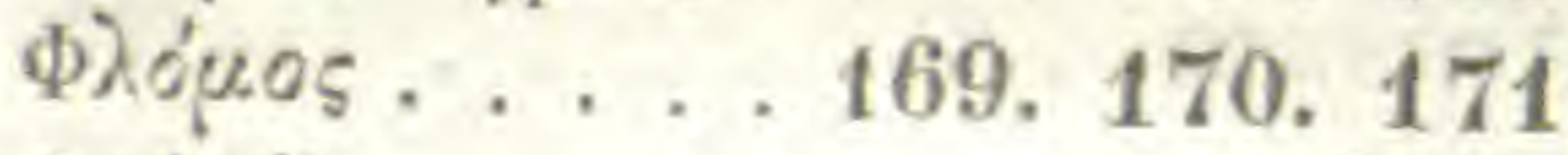

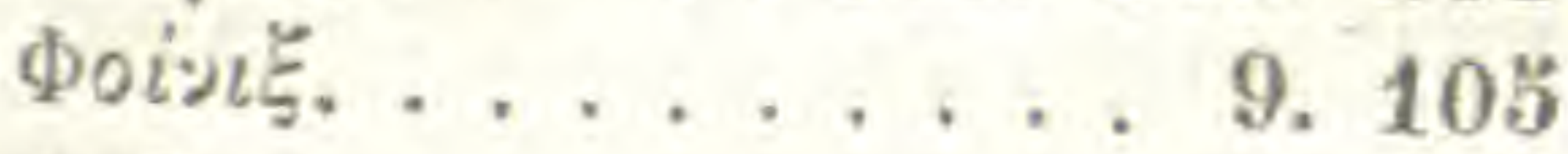
Фоนี. . . . . . . . 46 Фúxos $\theta \alpha \lambda \dot{\alpha} \sigma \varsigma\llcorner 0 \nu . . .265$ $\Phi v \lambda \lambda i \tau t \varsigma \ldots . . .263$

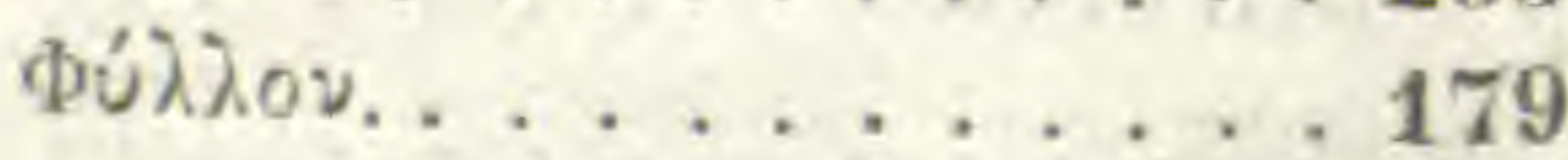

Xараúxтn.......134

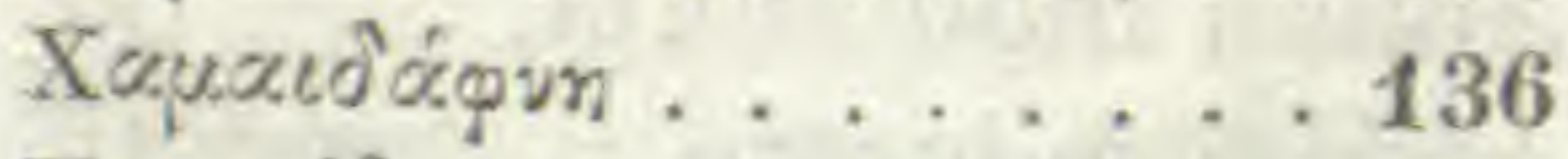
Xapaiopus. ....... 130

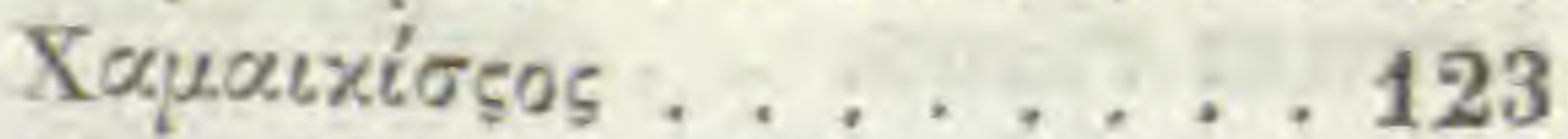

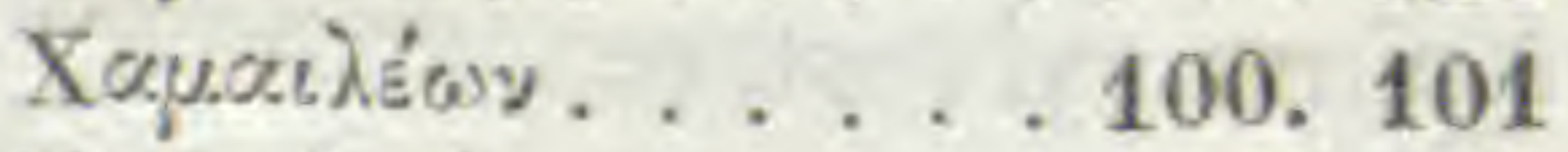

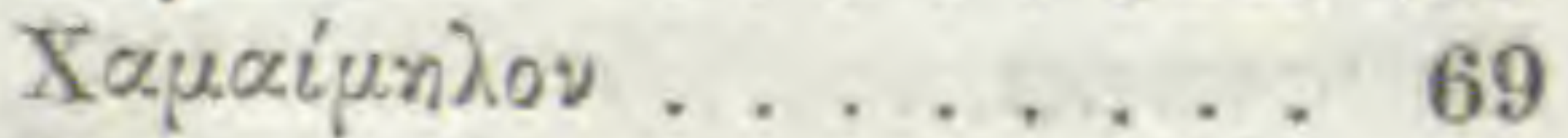

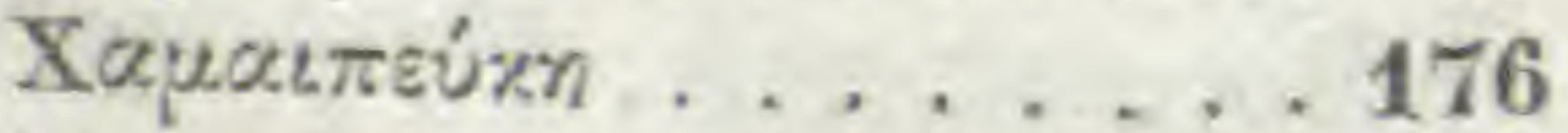

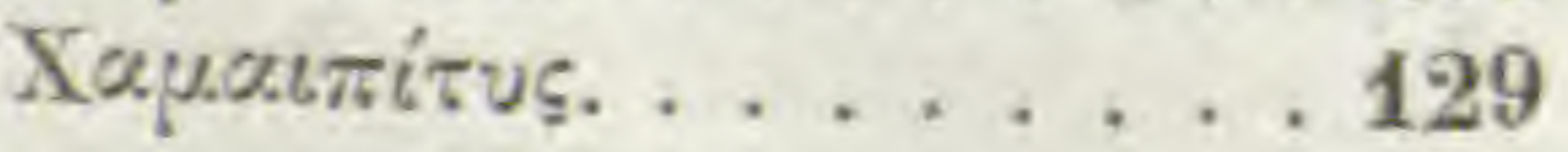

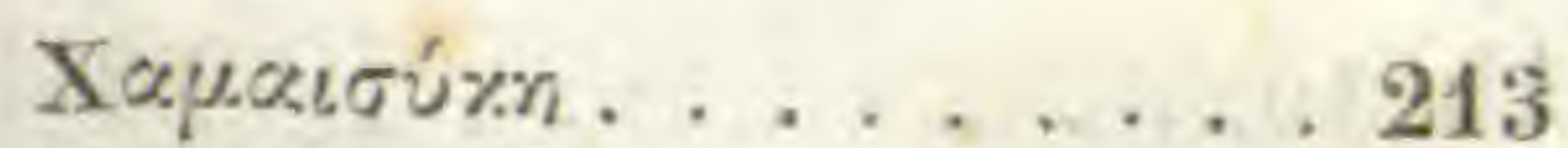

$\mathrm{X} x \mu \varepsilon \lambda \alpha i \alpha . \ldots . . .13 \mathrm{.}$

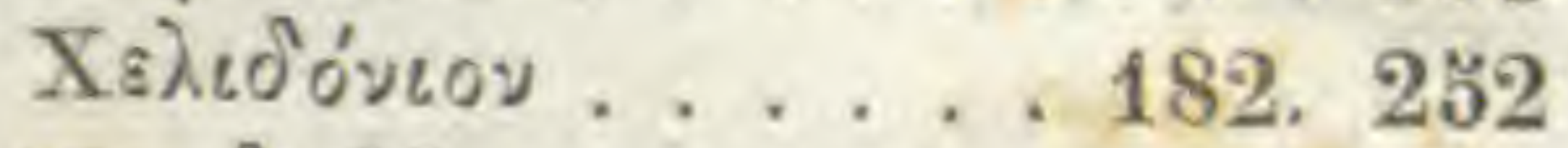

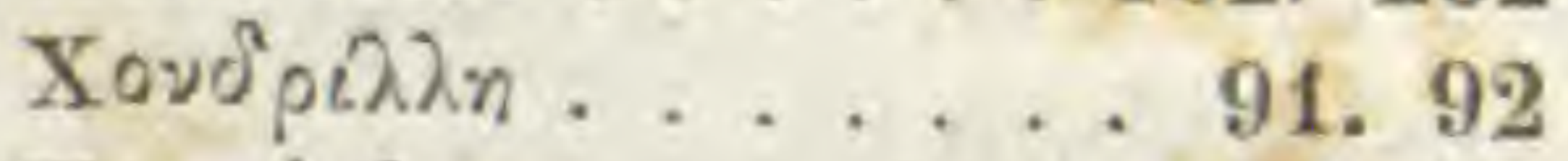

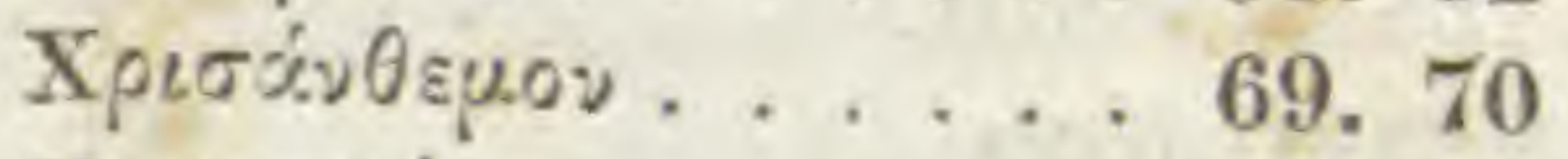
Хрибоко́н....... 79. 80

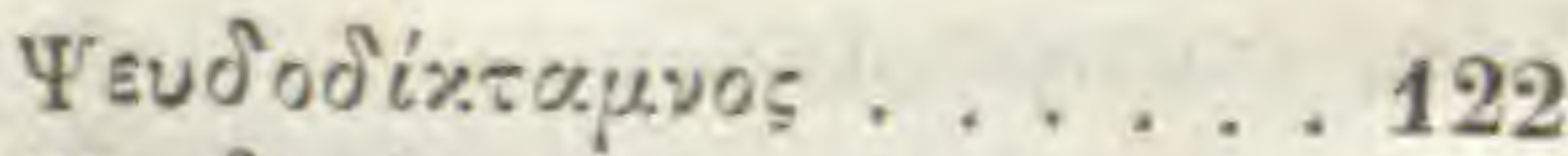

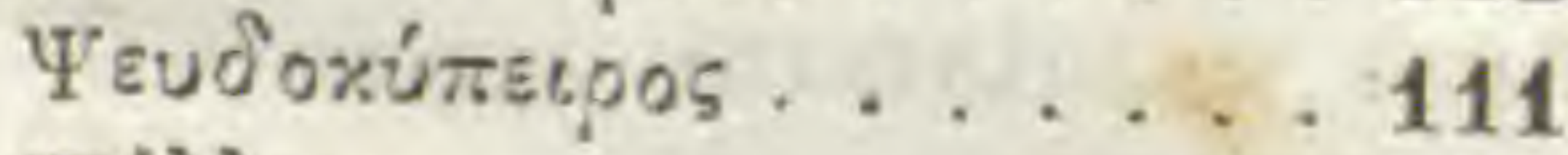

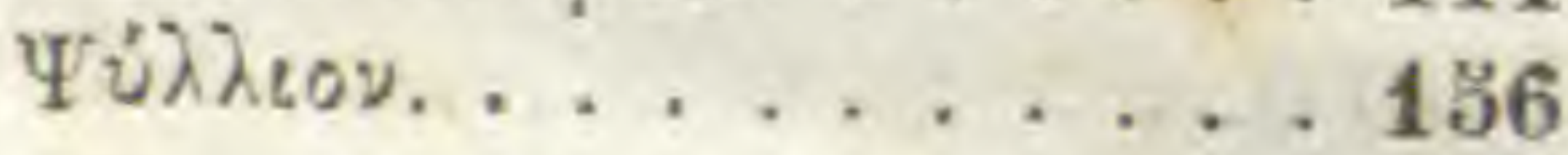

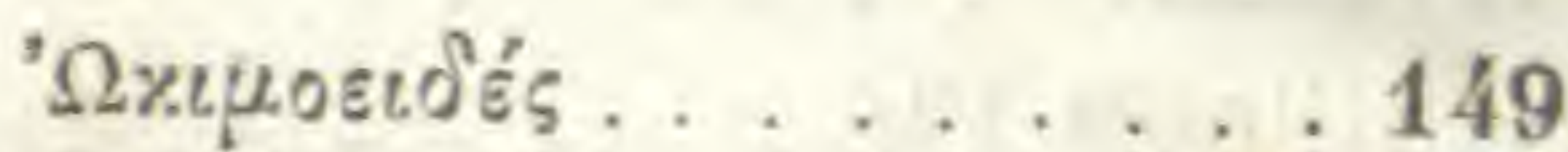
$\Omega$ хpós ........... 168 
Abeto........ 10

Abies........ 10

Abrotonum, .... 78

Absinthium. .... 77

Acanthium. . ... 93

Acanthus. . .... 140

Acer campestre. ... 5

Acer montanum. . . . 3

Acero........ 3

Acetosa. ..... 59

Achilleon Pli. . . . 183

Acicula. . . . . . 257

Aconitum. 199.200.249.250

Acorna. ...... 94

p Acoro. ...... 220

Acus muscata. ... 257

Adiantum. .... 264

Agylops. . . . . 104

Era. ....... 104

Ethiopis. . . . 113

Affodillo..... 215

Ageraton. ..... 76

Aglo di serpe. . . . 224

Agno casto...... 18

Agretti. . . . . . 198

Agrifoglo.

18

Agrimonia. . . . 132

Aingu ....... 129

Alaternus. ...... 11

Alberga. . . . . . . 193

S. Alberto. ..... 193

Alcanna ...... 11

Alcea. ........ 242

Alchechengi. . . . . 143

Alchimilla. . . . . . 6 aั

Alectorolophos.. . . . 113

Alfalfa.
Alga. . . . . . . . 26 כ

Alicacabum. ..... 143

Alleluya, . . . . . 233

Alliaria. . . . . . . 201

Alopecuros. . . . 106

Alsine. . . . . . . . รั

Althæa. . . 242.243.245

Alypum. . . . . . . 214

Alyssos Gali. . . . . 120ั

Alyssum. . . . . . 196

Anarago....... 90

Ambrosia. ..... 57

Ameos. ....... 21

Amellum. . . . . . . 178

Ammi. . . . . . . . 21

Ampeloprasum. . . 224

Anagallis. . . . . . 153

Anagallis aquatica. . . 173

Anagallis sylvestris. . 174

Anagyris. . . . . . 13

Anchusa. . . . . . . อั3

Androsæmum. . 227.228

Anemone. . . . . . 252

Angelica. . . . . . . 42

Anisum. ........ 31

Anthemis. . . . . . . 69

Anthyllis. . . . . . 73

Antirrhinon. . . . . 174

$p$ Antirrhino. . . . . 176

Anonis. . . . . . 159

Aphaca montana.. . . 168

Apium montanum. . . 36

Apium palustre. . . . 25

Apium risus. . . . . 250

Apium sylvestre. . . . 39

Apocynon. . . . . . 186

Appio......... 25 
Aquilegia. . . . . . 260 Arabis. . . . . . . 197 Archangelica. . . . 236 Arcion. . . . . . . . 102 Arctium. :.... 84 Argemon. . . . . . . 132 Argemonia . . . . 2237 Arisaron. . . . 141. 142 Aristalthæa.. . . . . 24อั Aristolochia. . . . . 231 Arnoglossa. . . . 456. 157 Aron. . . . . 141. 142 Artemisia.. . . . 75. 76 $p$ Artemisia. . . . . 77 Asarum. . . . . . . 229 Asclepias. . . . . . . 185 Ascyrum. . . . . . . . 227 Aspalathus. . . . . . 9 9 Asparagus sylvestris . 139 Asplenum. . . . . . 264 Asphodelus. . . . . . 215 Aster atlicus. . 71. 72:73 Astragalus. . . . . 167 Assenzo. . . . . . . . 77 Athanasia. . . . . . 75 Atractylis. . . . . . 99 98 Atriplex sylvestris. . 60 Avellana. . . . . . . . 2 Avornello . . . . . . 13 Auricula muris. . . 173 Azaruolo salvatico. . . 15

Baccara. . . . . . 229

Baccharis. ..... 45 Baciuco. ...... 44 Ballote. . . . . . 121 Balsamina.
Barba iovis. ..... 12 Barba sylvana. . . . 244 Bardana. ...... 102 Batis. ....... 44 $p$ Been albo. .... 152 $p$ Been rubro. . . . . 58 Bellis......... 74 Berberi. . . . . . 15 Betonica. 116.117.119.120 Bislingua. . . . . . . 136 Bismalva. . . . . . . 243 Bistorta. . . . . . 58 Blattaria. . . . . 171 Blitum. ....... 56 56 Bocea di lione. . . . 176 Bonaga. . . . . . . . 159 Bonifatia. . . . . 136 Bosso.. . . . . . . . 16 Botrys. . . . . . . 57 Branchorsina. . . 140 Brassica canina. . . . 60 Brassica marina. . . 190 Bromos. . . . . . 104 Brusco. . . . . . 136 Bryonia. . . . . 147 Bryon marinum. . . . 265 Buglossa hispana. . . 54 Bulbus vomitorius. . 217 Buphthalmum. . . . 70 Bursa pastoris. . . . 197 Butomus. . . 2 240.241 Buxus. . . . . . . 16

Cacalia........ 82 Cacatreppolı... . . . 9 96 Calamentum. ...... 126 Calla. ........ 51 
Canapa........ 63

Canapaccia. ...... 77

Canforata.

76

Canna d'india. . . . . 222

Cannabis sativa.

63

Cannabis sylvestris. . 242

Capparis. . . . . . 231

Capelvenero. . . . . 264

Capillus veneris. . . 264

Capucci fiori..... . 260

Caracias. . . 209. 212

Caranza. . . . . . 206

Carara. . . . . . 197

Cardiaca. . . . . . 192

Carduus sylvestris... 101

Carlina. . . . . 101. 107

Carpesium. . . . . 46

Carpinus. ....... 2

Carrubbio. . . . . . 13

Cartamus.

Casia Vergilii.

98

Cassutha.

234

Cataputia. 130

Cauda leonis

212

Cauda vulpina.

Cauta.

Cedornella.

Cedrus. 149

Celidonia.

Celtis. 182

Centaurium.

Centaurea. 183

Centinodia. 183

Centonghio. ธั6

Centunculus.

Centumgrana.

Centum capita.
Cepaea. 261. 262

Cerfoglo. 38 Cerinthe. 51 Cetrach. 264 Chamsecissus...... 123 Chanıæyparissus. . 66 Chamsedaphne. ... 136 Chamaedrys. .... 130 Chamæleon. .. 100. 101 Chamæmelun.

Chamæenee. 176 Ghamæepitys. 129 Chamæsyce. 213 Chamælea. $13 \mathrm{~s}$ Chamomills. 69 Chærefolium. . . . . 38 Chelidonium maius. . 182 Chelidonium minus. . 2อั Chiarella. ... . 113. 114 Chironium. . . . 86. 230 Chondrilla. . . . 91.92 Chrysanthemum. . 69, 70 Chrysanthemum Democriti. . . . . . 25ั5 Chrysocome. . . . 79.80 Cicera. 168 Cicerbita 88 Cicuta. 36 Cineraria. 76 Cinquefoglo. . . . . 239 Cipolla di serpe. . . . 217 Cirsium. 99. 100 Cisthus. 234 Clematis. . . . 184. 237 Clinopodium. 127 Cneorum. 234 Cnicus. 
Cocomero asinino. . . 206 Colchicum. ......221 $p$ Colocasia. ..... 142 Colutea........ 12 Connina.

Consiligo....... 218

Consolida maggiore. . 52 Convolvulus. . . . . 189 Conyza. . . . . . . 87 Corallina. . . . . . . 265 Coris. 228 Corniola de tintori. . 158 Coronopus. . . . . 157 Correggiola. . . . . 56 56 Cotula fæetida.. . . . . 69 Cotyledon. ..... 262 Costola d' Asino. . . . . 92 Crassula maior. . . . 262 Cratægus....... 15 Crepis. ........ 92 Crista gallinacea. . . . 143 Crithmum. ....43. 44 Crocum sylvestre. . . 221 Crucialis. . . . . . . 203 Cruciata. . . . . 181 Cucubalum. . . . . . 145 Cucumis sylvestris. . 206 Cuminum sylvestre. . 260 Cuscuta. ...... 430 Cyanus. . . . . 100. 102 Cyclaminus. . . . . 230 Cynoglossa. ...... 50 Cynocrambe. .....60 60 Cynosorchis. . . . 225 Cyparissias, . . . 209. 212 Cyperus. . . . . . 109. 111 Cyprus.
Cytisus.

Daneda. . . . . . . . 75

Daphnoides. ..... 135

Daucum. . . . 32.31. 34

Delfinium.

195

Dendrodes. . . . . 210

Dentaria. . . . 199. 200

Dente cavallino. . . 149

Dictamum cretease. . 129

Digitalis. . . . . . 173

Diosanthos. . . . . 151

Dipsacus. . . . . . . 95

Dittamo bianco. . . 259

Doria. . . . . . . . 86

Doronicum. . . . . . 71

Dorycnium. . . . . 190

Draba. ...... 197

Dracon. ....... 74

Dracunculus. ....141

Dragontea. ..... 141

Dryopteris. . . . . 263

Ebbio.

134

Ebulus. . . . . . . 134

Edera spinosa. . . . 138

Edera terrestris, ... 123

Elabro. . . 245. 247.248

Elaphoboscum. . . . 28

Elatine. . . . . . . . 229

Eleoselinon ...... 25

Ella . . . . . . . 71

Elleborum. 246. 247. 248

Elleborine. . . . . 248

Elxine cissampelos. . 189

Empetrum. . . . 43. 44

Enneaphyllon. . . . 247 
Enula campana.

Ephemerum. . . 221. 226

Epimedium. 229

Epipactis. . . . . . 248

Equisetum. . . . . . 68

Erica. . . . . . . 7.8

Erigeron. . . . . . 79

Ervilia. . . . . . 169

Eryngium.. . . . 94.95

Erysimum. . . . . . 495

Erythrodanum. . . . 203

Erythronium. . . . . 219

Esula. . . . . . 209. 211

Eufragia. . . . 178. 180

Evonymus. . . . . . 1

Eupatorium. . . . 132 132

Eupatorium Mesues. . 183

$p$ Eupatorio Mesues. . 76

EupatoriumAvicennæ. 85

Faba inversa. .

229

$p$ Faba inversa. . . . 262

Fagus.

Faggio.

Favagello.

1

Favagrossa.

252

Farfara. 262

Felce.

81

266

Fendesassu. 44

Festuca. 104

Fidicula. 264

Fiengræco. 159

Fil' di spada. 221

Filipendula. . 238

Filix 266

Finocchiella. 37

Fioraliso.
Fior' capucci. 260

Fior' gharofano salvaticho. 151

Flammea...... 230

Flammula. ..... 237

Fluida.

Foenugræecum. . . . 159

Formentone. .... 62

Frassinella..... 137

Frassinello...... 259

Frassino. ..... 3

Fraus. . . . . 89

Fraxinus. ..... . 8

Fucus marinus. .. 26ã

Fusano........ 1

Galiopsis...... 172

Gallion. ...... 203

Gariofilata, ..... 237

Gattaria. ..... 126

Gattero. ..... 6

Genista. . ..... 9

Gentiana. . . 180. 181

Geranium. . . . 36. 298

Ginestra. . . . . . 159

Ginestrella...... 89

Ginepro. .... 7. 10

Gingidium. ..... 34

Gigaro. ....... 141

Girasole. . . 207. 208

Gittone....... 151

Giuggiolo.

Gladiolus. . . . 221. 222

Glastum. ....... 48

Glaux. ....... 163

Gnaphalium. ..... 154

Gralega ...... $16 \mathrm{a}$ 
Gramen aculeatum. . 107 Gramen harundina-

ceum. . . . . 106

Gramen Parnasium. 154 Gratia Dei. . . . . . 183

Gruogo saracinesco. . 98 Guado. .........48 48 Guaraguasco. . 169.170

Halimus.

Halimum Hermolai. . 205 Hastula regia. . . . 215 Hedera terrestris. . . 123 Heleninm. : . . . 71 Helenium Egyptium . 164 Helichrysum. . . . 79. 80 Helioscopius. . . . . 214 Heliotropium. 50. 182,207 Hemerocallis. .... . 217 Heptaphyllon. . . 239. 240 Herba cancri. . . . . รั0 Herba gatta. . . . . 126 Herba Lucciola. . 103.142 Herba mora. . . . . 123 Herba Moscada. . . . 257 Herba Paris. . . . . 233 Herba Pepe. . . . 198 Herba Roberti. . . . . 257 Herba s. Alberti. . . . 195 Herba s. Jacobi..... 76 Herba s. Laurentii. . 123 Herba s. Maria..... 81 Herba Sardoa. . 250. 251 Herba Venti. . . . . 252 Herba stella. . . . . 157 Hermodactylus... . 221 Hiberis. 197. 198
Hieracium. ..... . 88

Hippolapathum. . . . 59

Hippuris. . . . . . . 68

Holostium. . . . . . 157

Horminum. . . . . . 145

Hyacinthus. . . . . . 218

Hydropiper. . . . . 64

Hyoscyamus. . . . . 149

Hypericum. . . . . . 227

Hypoglossa. . . . . 136

Hyssopum. . . . . . 128

$p$ Hyssopo. . . . . 128

$p$ Hyssopo montano. . 127

Iacea. . . . . . . 230

S. Iacobi ....... 76

Imperatoria. . . . . 35

Impia. . . . . . . . 79

Incensaria. . . . . . T2

Inguinalis. .....74 71

Iovis flos. . . . . . 451

Irio. . . . . . . . 198

Iris. . . . . . . . 220

Isatis. . . . . . 48. 57

Ischremon. . . . . 103

Iva. . . . . . . . 129

Iniuba. . . . . . . 49

Iuncus. . . 108. 109. 112

Iuniperus. . . . 7. 70

Iusquiamo. . . . . . 149

Labrum veneris. ....99

Laburnum. . . . . . 13

Lac avium. . . . . 223

Lactuca sylvestris. . . 91

Ladano. . . . . . . 234

Lagopus.
161 
Lamium. . . . . . 117

Lanciola. . . . . . . . 456

Lantana. . . . . . 17

Lapathum. . . . 58. 59

Lappa canaria. . . . 132

Lappa maggiore. . . . 102

Lappa minore. . . . . 207

Lapsana. . . . . . . 193

Laserpitium. . . . 26.42

Lathyris. . . . . 212

Lattaiola. . . . . . . . 90

Latte di gallina, ... 223

S. Laurentii. . . . . 123

Laurentina. . . . . . 123

Laureola. . . . . . 135

Laurus alexandrina. . 136

Laurus regia. . . . . 19

Laurus sylvestris. . . 18

Lavanese. . . . . . . 165

Ledon. . . . . . . 234

Lenticula palustris. . 263

Lentiscus. . . . . . . 14

Leontopetalon. . 181. 182

Lepidium. . . . . . 198

Leporaria. . . . . . 161

Leporis auricula... . 164

Leporis palatium. . . 439

Leporis pes. . . . . 161

Leucas, . . . . 417. 121

Leucoion sylvestre. . 200

Levistico. . . . . . 44

Libanotis...... 22. 72

Lichen. ........ 68

Lichnis. . . . . 151. 152

Ligusticuns. . . . 28. 28

Ligustrum. . . . 14.17

Lilium convallium. . 138
Lilium sylvestre. . . 219

Linaria. . . . 176.177

Lingua avis . . . 3. 154

Lingua canina. . . . . 50

Lingua cervina. . . 263

Linozostis. . . . 192

Linum sylvestre. . . 232

Lithospermum. . . . 5 54

Lolium sylvestre. . . 105

Lonchitis aspera. . . . 263

Lotus arbor. . . 19. 20

Lotus sylvestris. . . 160

Lucciola. . . 103. 142

Lunaria. . . . 201. 204

Luparia. . . . . . 250

Lupinum sylvestre. . 166

Lupulo. . . . . . . 63

Lupus salictarius. . . 63

Lycochtonon.199.200.249.

250

Lycopsis. . . . . . . 54

Lysimachia. . . 118. 155

Madreselva. . . . . 228

Madrisalvia......415

Maggiurana. ..... 128

Magydaris. . . . . 26

Malope......... 243

Malva. .......244

Malvavischio. . . . 243

Malvone. ...... 243

Mampé. ...... . 234

Mandragoras. . . . 148

Marrobbio. . . . 122

Marrubium. . . . . 122

Marrubium nigrum. 121

Martagon. 
Marum.

128 228

Matrisylva. 138 $p$ Matrisylva. 75 Matricaria. Medica. 160. 161 Medion. ... . . 235. 236 Melanthium. ......233 Mela spinosa. ..... 147 Melilotum Serapionis. 163 Melilotus. ...... 81 Melino. . . . . . 146 Melissa. . . . . . 119 Melissophyllon. .... 119 Melancranis. .... 112 Menta acquaiola... 127 Mentastrum. ..... 127 Mercurialis. . . . . 192 Mercorella. . . . . 192 Mespilus sylvestris. - 15 Meon......... 21 Meu. ........ 24 Mezereon. ..... 135 Migl' alsole. . . . . . . 54 Milium solis. . . . . 5 54 Millefoglo. . . .... 80 Millefolium stratiotes . 80 Milzadella. . . . . 121 Moli. ........ 224 Morella. . . . . . . 123 Mortella. . . . . . . 16 Morsus Diaboli..... 131 Mughetti. . . . . 138 Muris auricula. . . . 173 Muscus marinus. . . . 265 Myosotis....... 173 Myrica.

Myriophyllon. . . 119. 155
Myrrhis. ....... 37 Myrtus. . ...... 16 Myrtites. . . 209.210

Napello. . . . . . . 249

Nardum montanum. . 430

Narcissus. . . . . 218

Nasso.. . . . . . . 10

Nasturtium. . . . . 198

Nenufaro. ...... 232

Nepa. . . . . . . 158

Nepita. . . . . . 126

Nerion. . . . . . . 185

Nigella. . . . . . . 233

Nummularia. . . ..... 4 อัว

Nux pontica. ...... 2

Nymphæa....... . 232

0chrus. . . . . . 168

Ocimastrum. ..... 149

Ocimoides. . . . . 149

Oculus bovis........ 70

Odontides. ...... 152

Oenanthe. ....... 238

Oleandro. ...... 485

Olivella. . . . . . . 1355

0nagra. ..... 187. 188 Onobrychis. ......165 Ononis........ 159

Ontano......... 5

Ophioglossa. ..... 142

Ophioscordon. ..... 224

Ophrys. . . . . . . 226

Oppio. ........ 5

Opulus. ........ 5

Ordeum murinum... 105 Orecchio di lepre. .. 164 
Orecchio di lupo. . . 457 Oreoselinon. ..... 38 Origanum. . . . . 128 Oriola. 135

Ornithogalon. . . . . 223 Orobanche. . . . . . 179 Osyris. . . . . 175 Oxalis. . . . . . 59 Oxyacantha. .... 45 Oxycedrus....... 7 Oxymirsine.. . . . 436 Oxys. 233 Oxyschoenus. 112

Pronia. 259

Palatium leporis. 139

Paliurus africana.

Palma.

Palma christi. 225

Panacea.

Panax asclepium.

Panax chironium. 86.230

Panicum sylvestre. . 108 Pan' porcino. . . . 2 230

Papaver corniculatum, 181

Papaver rhoeas. . . 233

Papaver spumeum. . 150

Papyrus. . . . . . 110

Paralius. . . . . . 210

Paris. . . . . . . 233

Paronychia, . . . 5รั

Parthenium. . . . 69

Pastinaca.... . 31. 33

Pastricciano..., 31. 34

Pecten veneris. . . . 257

Pelecinus. . . . . $166^{3}$ Pentaphyllon.
Peplis. 213

Peplium. 213

Peplus.

Perfoliata.

Perforata.

Periclymenum. . . 138

Periploca.

Peristereon.

Persa.

Persicaria. 210. 214

Personata. 64

Pes anserinus. . 102

Pes columbinus.

Petasites.

Petroselinon,

83

Peucedanum.

Phalangium.

Phalaris. 216

Pharnaceon. . . 85. 86

Phillyrea.

Philyra.

Phlomis. . . . . . . 449

Phoenix. 105

Phu. 46

Phycos. ....... 265

Phyllitis. 263

Phyllon. 179

Piantandomino. 170

Picea.

Pie columbino. . . . 258

Pie corvino. . . . . 256 Pilatro.

Pilosella. . . . . . 88

Pimpinella minor. . . 36

Pimpinella romana. 40

Pioppio.

Piperaquaticum. 
Pityusa.

212

Planta domini.

170

Plantago..... 156. 457

Plantago aquatica. . . 241

Platyphyllos. . . . 211

Polemonia... . . . 152

Polium.

129

Politrico.

264

Polygala.

463. 164

Polygonaton... . . 137

Polygonum. . . .56.67

Polypodium. . . . . 263

Populus. 6. 7

Porro salvatico.

224

Poterium.

158

Potamogeton.

133

Prasium.

122

Provinca. 184

Prunella.

123

Pseudocyperus.

Pseudodictamum. 114

Psyllium. 129

Ptarnica. 156

Pugnitopo. 73. 74

Pulegium. 136

Pulicaria. 127

Pulmonaria. ... 51. 65 Pulsatilla. 250

Pyrethrum. 40

Pyrola. 204

Querciola. 130 Quinquefolium. 239

Radicetta salvatica. . 193 Rafano. 196 Ranunculus.250. 231. 253. 254. 2 รั้ว. 2 อิ 6
Raperonzo. 235 Rapunculus. . . . . 23: Reganı. . . . . . 128 Rena. . . . . . 26 Restabovis. . . . . 159 Rhamnus. ... ... 14 Rhododaphne. . . . 18. Rhus. . . . . : 14.17 Ricinus. . . . . 2. 208 Robbia. ...... 203 Robertiana. . . . . 257 Romice. . . . . . . 59 Rovistico. . . . . 11 Rostrum gruis. . . 258 Rubia. . . . . . 203 Rubiglo salvatico... 168 Rumex. . . . . . . 59 Ruscus. . . . . . 436 Ruta muraria. . . . 264

Sabina........ 8 $p$ Sabina. . . . . . 7 Sagapenum. ..... 23 Salvia minor. . . . 125 Sampsucum. . . . 128 Sanguinella. . . . 107 Sanicula.. . . . . 240 Santolina. .... 78 Santonicum. . . . . 77 Sancti Alberti. . . . 493 Sancti Iacobi. ... . 76 Sancta Maria. . . . 81 Sancti Laurentii. . . 123 Saponaria. . . 45. 150 Sardoa. . . . 250. 2อ1 Sassefrica. . . . . . 90 Satyrio. 225 
Satyrium erythronium. 219 Saxifraga.

Saxifraga alba.

179

Saxiraga Hermolai. . 153

Saxifragia. .

32

Satureia. .

128

Scabiosa. - 131. 132. 133

Scamonium maius..

191

Scandix.

257

Scariola.

91

Scardaccione.

95

Schianza.

112

Sclarea.

114

Scodano. .

Scolopendrium. . . 261

Scolymus. ...994.101

Scopa. . . . . . . 7.8

Scopa regia. . , , . . 144

Scordium. . . . . 130

Scorpius. . . 158. 205

Scorpioides. ..... 50

Scorpiurus. . . ... 182

Scrofularia. . 172. 252

Securidaca. . .... 166

Sedum. . . ...2.261

Sempervivum. . .... 261

Senapa.

Senecio,

Seriphium.

Sermollino.

127. 129

Sermontano.

Serpentina.

142

Serpyllum.

127. 129

Sertula campana. . 81

Sesamoides.

230

Seseli. . 27. 30. 35ั. 39. 40

Sideritis.124.125. 172.257
Sigillum Solomonis. . 226

Siler.

Siler aquaticum.... 38

Siler montarım . . . 27

Siliqua. ....... 43

Silio... . . . . . . 1

Silphium. . . . . 42

Sion. . . . . . 32

Siringa. . ..... 16

Sisimbrium. . . . 127

Sison. . . . . . . 40

Sinapis. . . . . 194

Smilax. ...... 10

Smilax aspera. . . . 438

Smilax Jævis, . . . 189

Solanum | 143.144.145.

Solatro 146.147

Soldanella. . . . . . 190

Sommacco. . . . . . 14

Sonchus. ... 88. 92

Sorba torminalis. .. 6

Sparganium. . . . 433

Spartium. . . . . 159

Sphondylium. ..... 24.

Spina appendix. . . 15

Spina sylvestris. . ... 9

Spin' cervino. . . . . 4

Splith. , . . . . . 181

Stachys, . . ..... 115

Staphisagria. . . . 249

Staphylinus. . . . 31. 33

Staphylodendron. .. 3

Stelephuros, ..... 106

Stellaria. . . . . 94.95

Stichados. . . . . 130

Stoechas. . . . 130

Storace. . 
Strafizeca. . . . . 249

Stramonia. . . . . 147

Stratiotes. . . , . 263

Stratiotes millefolium. 80

Strychnos. 143. 144. 145. 146. 147

Styrax.

$p$ Sycomoro. .

Symphytum.

Symphytum petræum. 129

Tamarigia. . . . . 8

Tamarix.

Tanacetum.

Targone.

Tasso barbasso.169.170.171

Taxus. . . . . . . 10

Terebinthus. . . . . 10

Tertiola. . . . . . 118

Testiculus canis. . . 225

Teucrium. . . . . 130

Thalietron.

47. 49

Thlaspi.

196

Thymbra. 128

Thymelrea.

Thymum.

135

$p$ Thymo.

128

Tignamica. . . . . . . 80

Tiglo. ....... 2

Tilia. ....... 2

Timiamica. .... 80

Tino. ....... 18

Tithymalus. 209.210.211.

212. 213

Tordylium.

Tormentilla,

32.40

Tragopogon.
Tragoriganum. . 121. 425 Tragos. . . . 10 10.205 Trasi. 109 Tribulus terrestris. 468 Trichomanes. ... 264 Tricoccum. ....207 Trifolium bituminosum. . . . . . 162

Trinitas. . . . . . 229

Tripolium. . . . . . . 58 Tripuli. . . . . . 160

Trissago. . . . 130 Turbith. . . 212. 214 Tussilago. . . . . . 81 Typha. . . . . 112

Valeriana. . . . . 46

Venti herba. . . . . 252

Veratrum. 246, 247, 258 Verbasculum. . . . 149 Verbascum. 169. 170. 171 Verbascum sylvestre. 118 Verbena. . . . . 120 Verbenaca. . . . 120

Verdemarco. . . . 49

Veronica. . . 174. 175

Verrucaria. .... 50

Viburnum. . . . . 17

Vilucchio. . . . . . 189

Vinca pervinea. . . . 484

Vincetossico. .... 485

Vincibosco. . . . 138

Viola flammea. . . 2230

Virga aurea. . . 85. 86

Virga regia. . . . . 173

Viticella. ..... 237

Vitis alba. . . . . 147 
Vitis sylvestris. . . 144 Vitex....... 48 Umbilicus veneris. . . 262 Urtica. . . . . . . 61 Urtica mortua. . . . 172 Uva d'india... . . . . 4 Vulparia. ..... 250

Xanthium...... 207

Xiphion. ........ . 221

Xiris. ....... 220

Zizypha. . . . . . . 19 Zafferano salvatico. . 221 


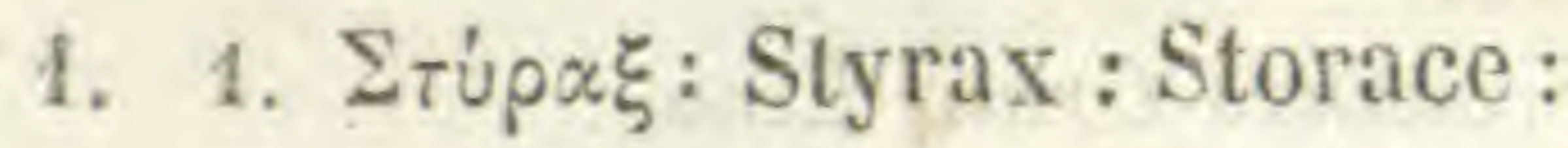

Lib. II, cap. 34 .

Ramus cum floribus. Styrax officinale Linn.

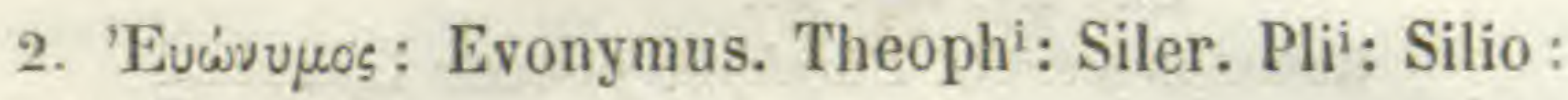
Fusano:

Lib. III, cap. 50 .

Ramus, omnino amissus, sed ab imagine impressa $E v o-$ nymus europaus Linn. bene cognoscitur.

3. Фทrós: Fagus: Faggio:

Lib. II, cap. 3.

Frustilla duo, cum reliquiis amenti mascu.i. Fagus sylvatica Linn.

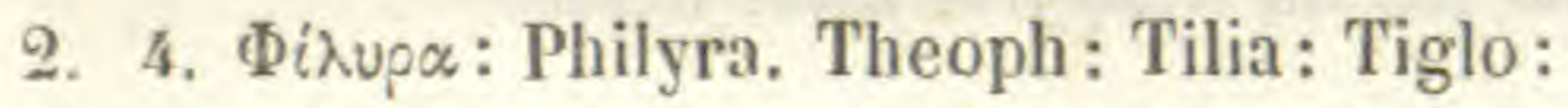

Lib. II, cap. 10.

Ramulus juvenis sterilis. Tilia grandifolia Ehrh.

5. Zuyia: Zygia. Theoph: Carpinus: Carpino:

Lib. II, cap. 7: Arbor Fago similis, fructu ex folliculis membranaceis constante.

Ramulus sterilis, cum amento fructifero sejuncto. Ostrya carpinifolia Scop.

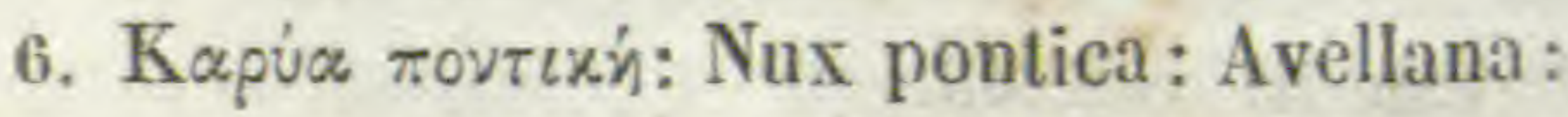

Lib. II, cap. 6.

Ramulus sterilis. Corylus Avellana Linn. 


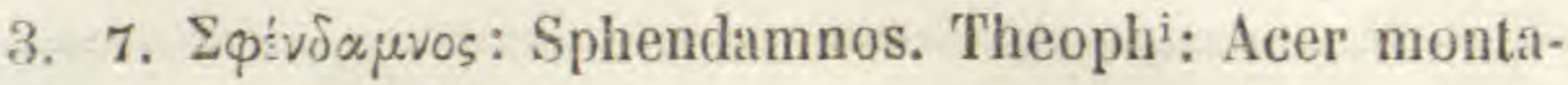
num : Acero:

Lib. II, cap. 12.

Ramulus cum racemo fructifero sejuncto. Acer PseudoPlatanus Linn.

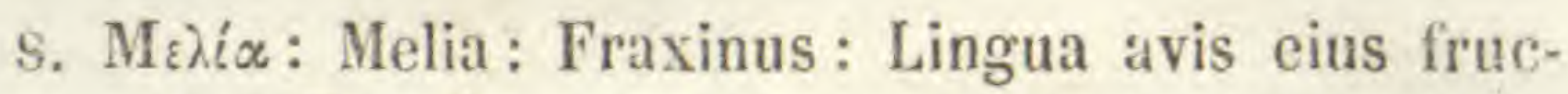
tus: Frassino:

Lib. II, cap. 13.

Ramulus fructifer. Fraxinus Ornus Linn.

9. Staphylodendron. Pli : Pistacchio salvatico:

Lib. III, cap. 44.

Ramulus sterilis. Staphylea pinnata Linn.

4. 10. Spincervino maggiore:

Lib. III, cap. 17: Spina Cervalis.

Ramus fructifer. Rhamnus catharticus Linn.

11. Spincervino minore:

Ramus fructifer. Rhamnus Frangula Linn.

12. Uva d'India :

Ramus sterilis. Diospyros Lotus Linn.

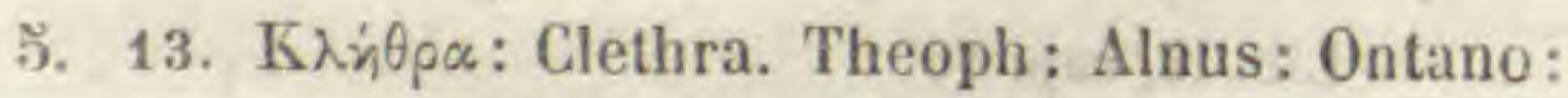

Lib. II, cap. 8.

Ramus sterilis. Alnus glutinosa Gærtn.

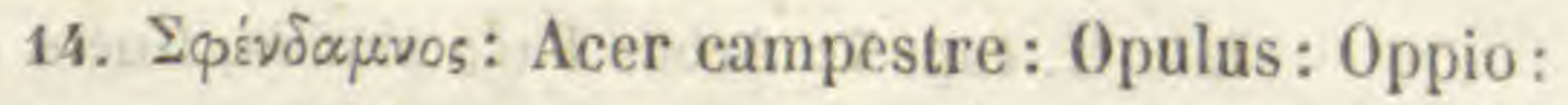

Lib. II, cap. 12.

Ramus sterilis. Acer campestre Linn.

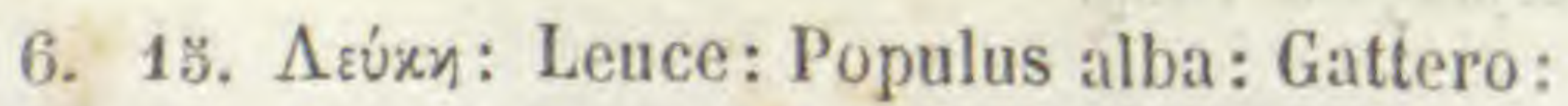

Lib. III, cap. 400 .

Duo tantum folia, lobata, sublus cinerea. Populus alba Linn. 
16. Sorba torminalis. Pli:

Lib. III, cap. 64.

Ramus sterilis. Pyrus torminalis Ehrh.

7. 17. Kédpos: Cedrus. Diosc: Cedrus Lycia. Theoph: Pro Sabina :

Lib. III, cap. 57: Sabina.

Ramus cum fructibus juvenibus. Juniperus phœenicea Linn. et auct.

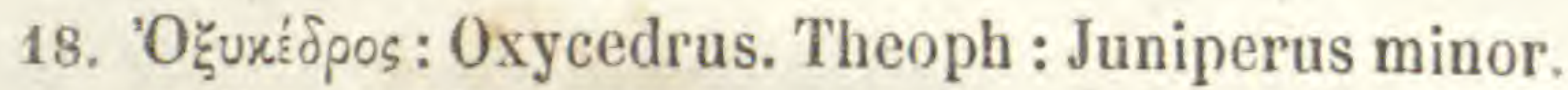
Diosc: Ginepro:

Lib. III, eap. 58, p. 137 : Juniperi alterum genus in marittimis, fructu nucis Avellanæ magnitudine, in maturitate flavo.

Ramulus cum aliquo fructu juveni. Juniperus macrocarpa Ten. (Fl. nap, non Sibth.); J. Osycedrus Endl. Syn. conif.

19. 'Airøıpos: Fgeiros: Populus nigra: Pioppio:

Lib. III, cap. 45.

Pènitus fere deficiens, folio tantum et vestigiis amenti fœrminei extantibus. Populus nigra Linn. et auct.

20. 'Eргixи: Erica: Scopa:

\section{Lib. III, cap. 20.}

Deest. Michelius determinavit per Scopam flosculis tanquam minuta granula virentibus, qua nequaquam dehiscunt Cæs. 1. c., vel Ericam majorem scopariam foliis deciduis Tournef. Inst., quæ Erica scoparia Linn. Sed ob habitum, formam et dimensionem imaginum foliorum, et præsertim sepalum adhuc reliquum lanceolatum, acutum, membranaceum, rufum, marginibus involutis, 4"' longum, mihi absque dubio $E$. carnea auct: videtur, qua sola inter Ericas Etruscas immo Italieas sepala ejusmodi habet. 
8. 21. Bpd́tus: Brathys: Sabina altera:

Lib. IV, cap. 9, p. 621 : Cneoron.

Rami duo, Passerina hirsuta Linn.

22. Mupixy: Myrica: Tamarix : Tamarigia:

Lib. III, cap. 47.

Ramus ut videtur fructifer, sed spicis omnino amissis.

Ex earum vestigiis tamen dijudicari potest, Tamaricem africanam Poir. potius quam gallicam Linn. esse; quod etiam confirmatur a specimine sequenti.

23. Tamarigia :

Lib. III, cap. 47: Myrica quædam sterilis, florem tantum ferens in spicis exilioribus.

Ramus florifer. Tamarix gallica Linn.

24. 'Eprixn: Erica: Scopa:

Lib. III, cap. 20: Scopa flosculis in quatuor particulas dissectis?

Ramus florifer. Erica arborea Linn.

9. 2ə. 'A $\sigma \pi \dot{\alpha} \lambda \alpha \theta 0$ : : Aspalathus: Spina sylvestris. Pli :

Lib. III, cap. 36.

Ramus cum reliquiis florum. Cytisus (Calycotome) spinosus Lam., Dec. Fl. fr. IV, p. 503.

26. Фoivt : Phónix: Palma :

Lib. II, cap. 46, p. 80 : Palma quæ in Italia seritur ex fructibus peregrinis quos Dactylos vocant.

Pars superior folii juvenis. Phcenix dactylifera Linn.

27. Genista:

Lib. III, cap. 35: Genista quadrato junco. Ramus cum reliquiis florum. Genista pilosa Linn. 
10. 28. "Apxєufos: Arceuthus. Theoph: Juniperus: Ginepro:

Lib. III, cap. 58: Juniperi genus in montibus, fructu magnitudine Myrthi, in maturitate nigro. Ramus fructifer. Juniperus communis Linn.

29. Diтus: Pitys: Picea: Sapino:

Lib. III, cap. 52, p. 130.

Ramulus sterilis, foliis fere omnibus lapsis. Abies excelsa Dec.

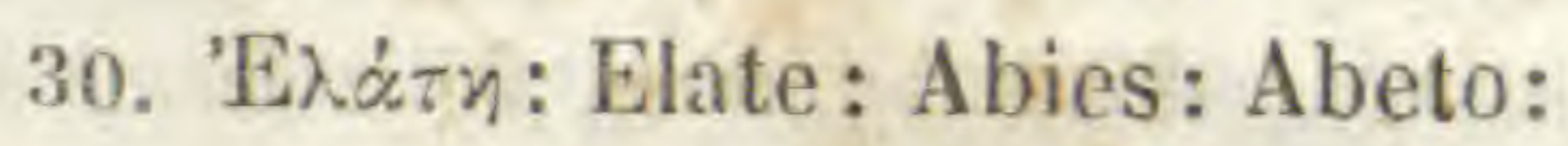

Lib. III, cap. 53.

Ramulus sterilis. Abies pectinata Dec.

31. $\Sigma \mu i \lambda \alpha \xi$ : Smilax: Taxus: Nasso:

Lib. III, cap. 54 .

Ramulus. Taxus baccata Linn.

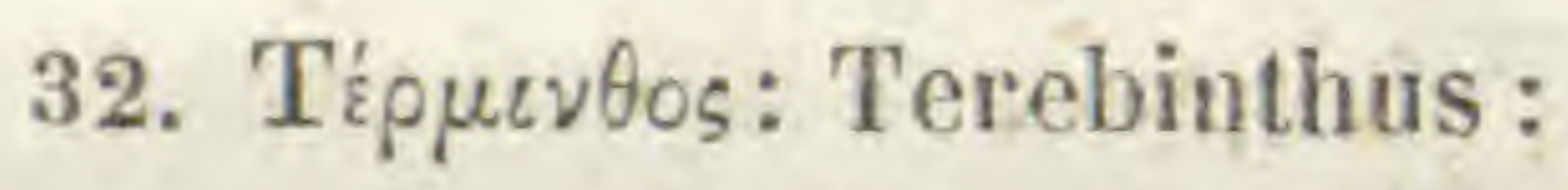

Lib. II, cap. 44.

Ramus fruetifer, gallam simul in foliolo ferens. Pistacia Terebinthus Linn.

11. 33, Alaternus. Pli:

Lib. II, cap. 36: Ilatrum foliis Oleæ.

Ramus florifer. Phyllirea media Linn. Sp. ed. 2, p. 11; Bert. Fl. ital. I, p. 40.

34. Kúrpos: Cyprus: Ligustrum: Alcanna: Rovistico:

Lib. HI, cap. 8.

Ramus fructifer, sed fructibus amissis. Ligustrum vulgare Linn.

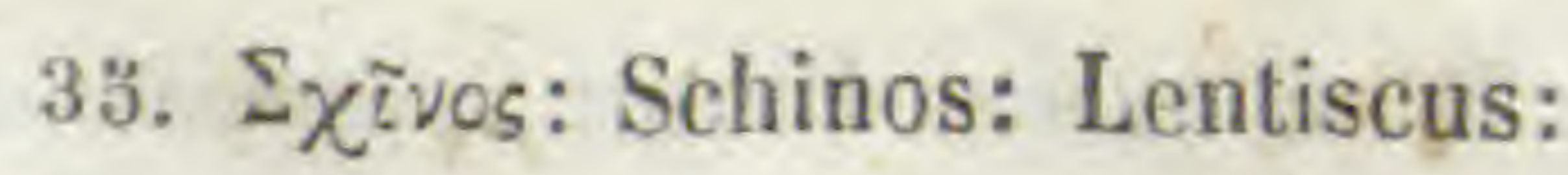

Lib. II, cap. 43.

Ramus fructifer. Pistacia Lentiscus Linn. 
36. $\Phi \iota \lambda \lambda \cup p^{\prime} \alpha$ : Phyllirea :

Lib. II, cap. 37.

Ramulus sterilis, Rhamnus Alaternus Linn.

12. 37. Koגout' $\alpha$ : Colutea. Theoph :

Lib. III, cap. 38: Emeri alterum genus.

Ramus florifer, cum fructibus ad latus. Colutea arborescens. Linn.

38. Kútıøos: Cytisus:

Lib. III, cap. 33: Cytisi sylvestris species in marittimis collibus albidior, foliis fermè sine pediculo hærentibus?

Ramus sterilis. Genista candicans Linn.

39. Barba iovis. Pli :

Lib. III, cap. 40.

Ramus florifer, Anthyllis Barba Jovis Linn.

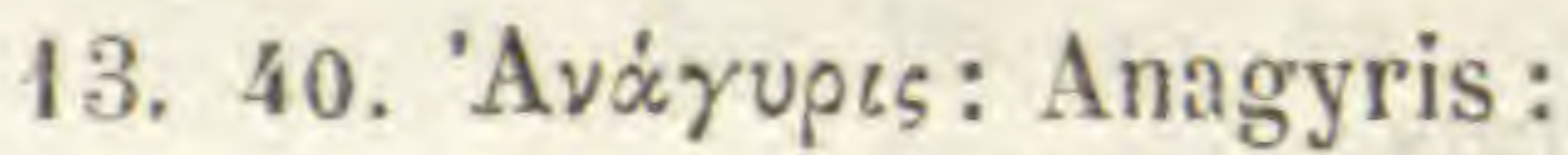

Lib. III, cap. 31 .

Ramus sterilis. Anagyris foetida Linn,

41. Laburnum. Pli : Avornello:

Lib. III, cap. 32.

Ramus florifer. Cytisus Laburnum Linn.

42. Scodano:

Lib. II, cap. 39.

Duo tantum folia. Rhus Cotinus Linn.

43. Kepwvi $\alpha$ : Ceronia: Siliqua: Carubbio:

Lib. III, cap. 27.

Ramulus sterilis. Ceratonia Siliqua Linn.

14. 44. Poũs 'Epulpos: Rhus rubea. Diosc: Sommacco:

Lib. II, cap. 42.

Ramus florifer. Rhus Coriaria Linn. 
45. P'́uvos: Rhamnus $p^{s}$ :

Lib. II, cap. 38: Rhamni primum genus.

Ramus sterilis. Jasminoides aculeatum, Salicis folio, flore parvo, ex albo purpurascente Mich! Nov. gen. p. 224, tab. 105, fig. 1; Lycium europceum Linn. Sp. ed. 1, p. 192; Mant. p. $47 ;$ L. mediterraneumDun. in Dec. Prodr.

46. $P \alpha$ น vos: Rhamnus $2^{s}$ :

Lib. II, cap. 38: Rhamni alterum genus.

Omnino fere deficiens; sed a vestigiis nullo dubio Hippophä̈ rhamnoides Linn.

45, 47. Azaruolo salvatico:

Lib. III, cap. 13: Oxyacantha.

Ramus florifer. Cratcegus Oxyacantha Linn.

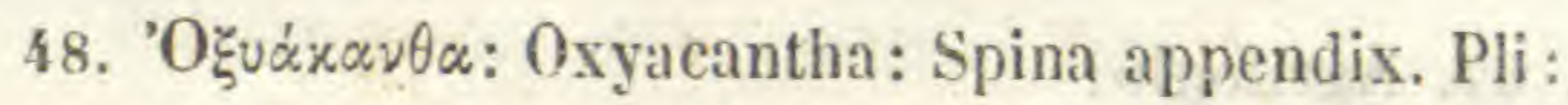

Lib. III, cap. 16: Lycium.

Ramus sterilis. Cratcegus Pyracantha Pers.

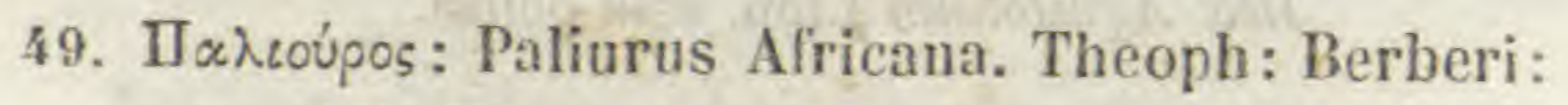

Lib. III, cap. 12 : Crespinus.

Ramus sterilis. Berberis vulgaris Linn.

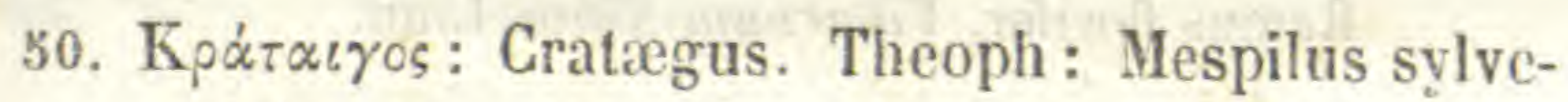
stris:

Lib. III, cap. 64: Sorbi alterum genus.

Ramus sterilis. Pyrus Aria Ehrh.

16. 51. Siringa :

Lib. III, cap. 9.

Ramus florifer. Philadelphus coronarius Linn.

32. Mupoivn: Myrsine: Myrtas: Mortella:

Lib. III, cap. 49.

Ramus florifer. Myrtus communis italica Linn, Sp. ed, 2. 
33. Пų่̌os: Pyxos: Buxus: Bosso:

Lib. III, cap. 48.

Ramus florifer. Buxus sempervirens Linn.

17. 34. Fluida altera:

Lib. III, cap. 4, p. 92: Alia Sambucus in paIustribus Pisanis.

Ramus florifer. Viburnum Opulus Linn.

55. Poũs: Rhus. Theoph: Fluida: Viburnum: Lantana :

Lib. II, cap. 44.

Ramus fructifer. Viburnum Lantana Linn.

56. Ligustro azurro:

Lib. III, cap. 43: Ligustrum Orientale.

Ramus florifer. Syringa vulgaris Linn.

18. 57. Agrifoglo:

Lib. III, cap. 6.

Defieiens; e vestigiis ramus fructifer. Nil aliad quàm Ilex Aquifolium Linn. esse potest.

58. Laurus syl. Pli : Tino:

Lib. II, cap. 60.

Ramus florifer. Viburnum Tinus Linn.

59. "A $\gamma$ vos: Agnos: Vitex: Agnocasto:

Lib. III, cap. 51.

Ramus florifer. Vitex Agnüs castus Linn.

19. 60. Laurus regia. Pli:

Lib. II, cap. 24.

Ramus sterilis. Prunus Láurocerasus Linn.

Laurocerasi descriptionem et figuram primus dedit Clusius in Rarior. plant. hist. p. 4, qui illam acceperat e Constantinopoli anno 1576, quare hoc anno in Europam allatam fuisse creditur, sed perperam, ut 
patet ex hoc herbario. Hanc arborem habuit Cæsalpinus e Genua ab horto Principis Doriæ, teste Bellonio in libello cui titulus: De neglecta plantarum cultura, cum Clusii Exoticis impresso, p. 239. (Cfr. Ant. Targioni Sulla introd, di varie piante nell'agric. ed ortic. tose. p. 237).

61. $\Lambda$ wtós: Lotus arbor: Bu (1) gratico.

Lib. II, cap. 52.

Ramus sterilis. Celtis australis Linn.

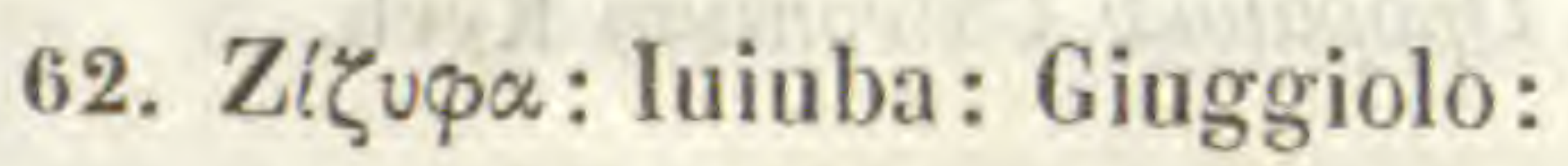

Lib. III, cap. 24.

Ramus florifer. Zizyphus vulgaris Lam.

20. 63. Lotus altera: Sicomoro:

Lib. III, cap. 23: Tuber.

Folium tantum. Melia Azedarach Linn.

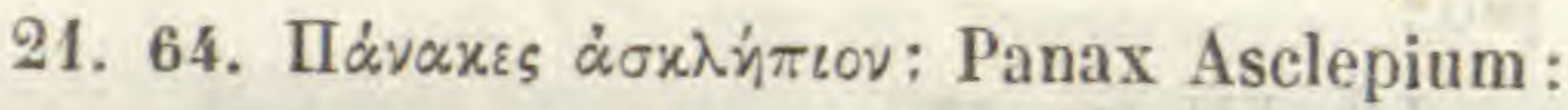

Lib. VII, cap. 4, p. 280 : Dioscoridis Asclepium ex Sicilia allatum?

Specimen floriferum. Anethum graveolens vel A. segetum Linn. et auct.

65. "A $\mu \mu \iota:$ Ammi : Ameos:

Lib. VII, cap. 15.

Specimen floriferum. Ptychotis verticillata Duby.

66. Mŕov: Meon: Meu :

Lib. VII, cap. 12.

Specimen fructiferum. Meum athamanticum Jacq.

22. 67. $\Lambda_{i} \beta$ avwris: Libanotis candida: Rosmarinum candidum:

Specimina duo superposita, superius floriferum sine

(1) Hic deficit littera, tarmele erosa, quam conjicere non potui. 
foliis, quod absque dubio Ferula Ferulago Linn., et Dec. Prodr. IV, p. 171; inferius e caulis frustulo foliifero constans ad Ferulam geniculatam Guss, ut videtur pertinens. Certe neutrum est Libanotis candida in lib. VII, cap. 7, quæ Prangos ferulacea Lindl. secundum Bert. Fl. ital. III, p. 459. An ad primum Panax Asclepium in lib. VII, cap. 4 referendum?

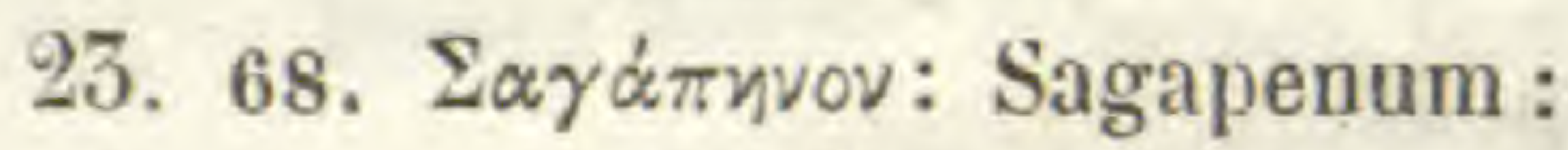

Lib. VII, cap. 50 : Herba Costa.

Folium tantum. Opopanax Chironium Koch.

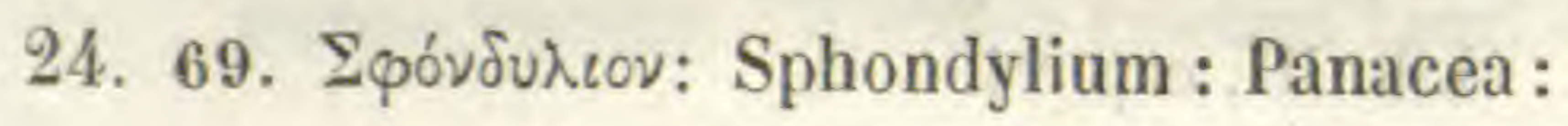

Lib. VII, cap. 54.

Specimen fructiferum cum folio radicali, sed fructibus omnino fere carens. Heracleum Pollinianum Bertol.

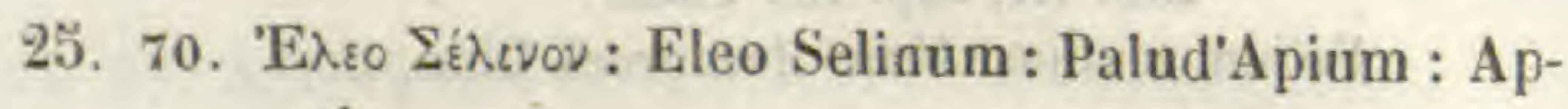
pio:

Lib. VII, cap. 38.

Specimen floriferum. Apium graveolens Linn. Sp.

71. Petroselinum sylvestre:

Lib. VII, cap. 42.

Specimen ut videtur floriferum, sed floribus prorsus deficientibus. Oenanthe pimpinelloides Linn.

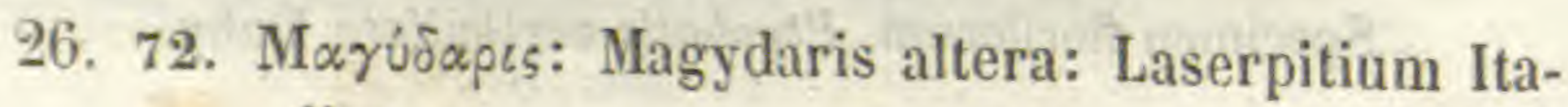
licum:

Lib. VII, cap. 44: Levisticum.

Folium tantum superest. Levisticum officinale Koch.

73. Laserpitium germanicum: Herba Rena :

Caulis et pars folii tantum supersunt. Laserpitium latifolium Linn.

Herba Rena in lib. VII, cap. 49 est verisimiliter Imperatoria Ostruthium Linn. 
27. 74. $\Sigma \dot{\varepsilon} \sigma \varepsilon \lambda_{\iota}$ : Seseli Massiliense: Siler montanum: Sermontano:

Lib. VII, cap. 32.

Folium. Laserpitium Siler Linn.

73. Пєux£́davos: Peucedanum:

Lib. VII, cap. 8: Libanotis nigra.

Folium. Peucedanum officinale Linn.

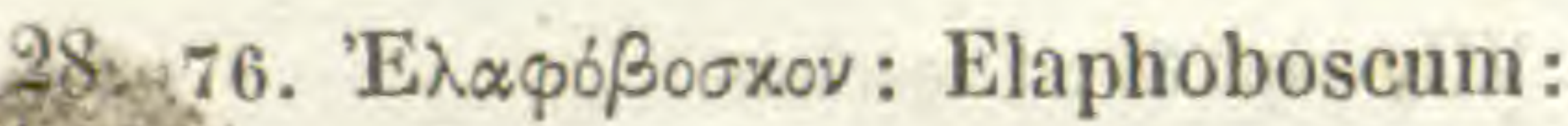

Folium. Videtur varietas Pastinacce sativa. Sub nomine Elaphobosci in lib. VII, cap. 56 describitur stirps Pastinacæ aliquantum similis, flore candido: quæ ista?

77. Ligusticum sylvestre:

Lib. VII, cap. 46 : Pseudoligusticum.

Specimen fructiferum. Aegopodium Podagraria Linn.

29. 78. Suúpvzov: Smyrnium:

Lib. VII, cap. 43: Olusatri alterum genus. Specimen ut videtur fructiferum, sed fructibus carens, cum folio radicali supposito. Smyrnium rotundifolium Mill.

30. 79. $\Sigma \varepsilon_{\sigma \varepsilon} \lambda_{c}$ : Seseli Ethiopicum: Perfoliata :

Lib. VII, cap. 34.

Specimen floriferam. Bupleurum protractum Link et Hoffm.

80. Perfoliata parva:

Lib. VII, cap. 34: Seselis Ethyopici genus parvum, foliis Graminis.

Specimen floriferum. Bupleurum Gerardi Jacq. 


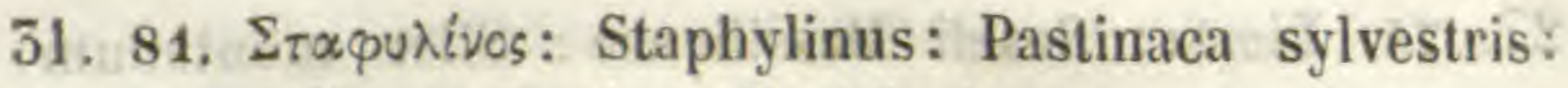

Dauco marino: Pastricciano:

Lib. VII, cap. 21.

Specimen fructiferum. Daucus Carota Linn.

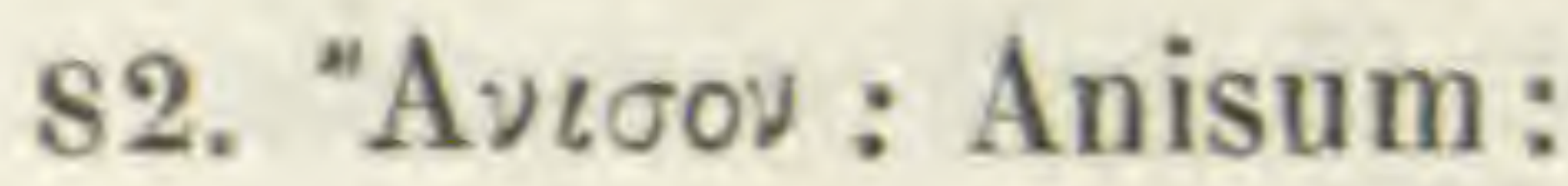

Lib. VII, cap. 60.

Specimen floriferum. Pimpinella Anisum Linn.

32. 83, Siov: Sion:

Lib. VII, cap. 39: Crescione.

Specimen floriferum, omnino fere deficiens. Helosciadium nodiflorum Koch.

84. Topoútıov: Tordylium alterum:

Lib. VII, cap. 58: Pimpinellæ Romanæ alterum genus.

Specimen fructiferum. Tordylium maximum Linn.

s.. $\Delta$ xข̃xos: Daucum tertium: Saxifragia vulgaris:

Lib. VIl, cap. 57: Saxifragia tertia.

Specimen floriferum. Pimpinella peregrina Linn.

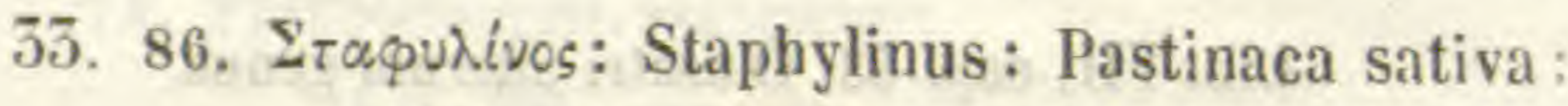

Lib. VII, cap. 55.

Specimen floriferum. Pastinaca sativa Linn.

34. 87. $\Delta$ ã̃xos: Daucum $\mathrm{p}^{\mathrm{am}}$ alterum: Dauco: Pastricciano:

Lib. VII, cap. 18.

Specimen floriferum. Ammi Visnaga Lam.

Huic etiam referri debet Cuminum quod Visnaga in lib. VII, cap. 14.

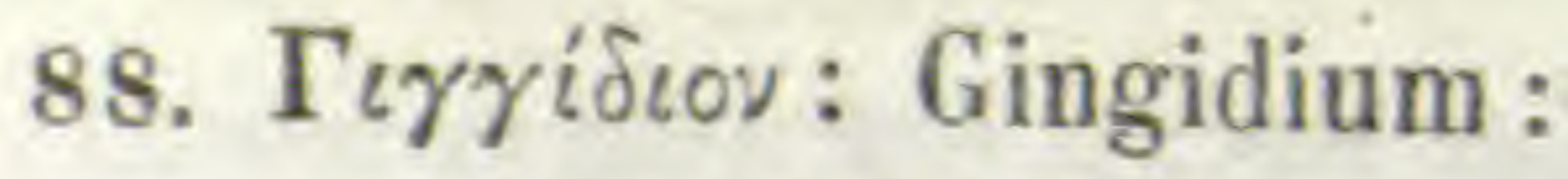

Lib. VII, cap. 22.

Specimen flores et fructus ferens. Torilis helvetica Gmel; Koch. Syn. ed. $2^{\mathrm{a}}$. 


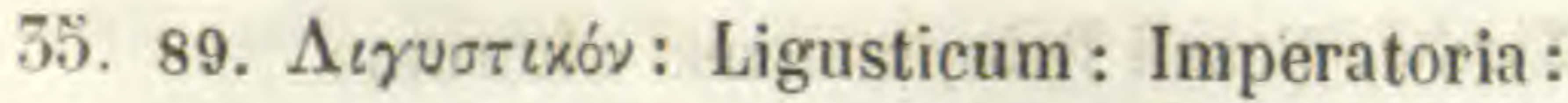

Lib. VII, cap. 46.

Folium. Trochiscanthes nodiflorus Koch.

90. $\Sigma \dot{\varepsilon} \sigma \varepsilon \lambda_{\ell}:$ Seseli Peloponnense:

Specimen floriferum. Peucedanum sulcatum (Selinum sulcatum Bert. Fl. ital. III, p. 367).

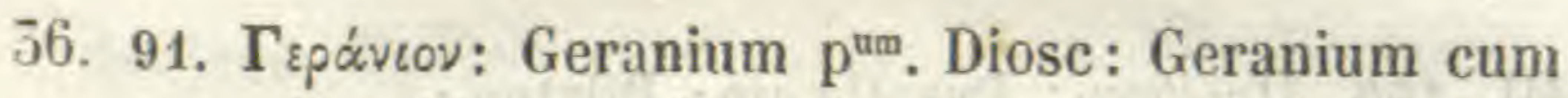
Myrrhide confusum radice rotunda dulci :

Lib. VII, cap. 29: Pancaseolus.

Specimen floriferum, valde mancum. Bunium Bulbocastanum Linn.

92. Kผ่ขยเоข: Conion: Cicuta:

Lib. VII, cap. 27.

Specimen floriferum. Conium maculatum Linn.

93. Pimpinella minor:

Lib. VII, cap. 57: Saxifragia altera minor.

Specimen valde mancum, partibus inflorescentiæ destitutum. Pimpinella saxifraga Linn. Sp, ed. 2a, p. 378.

37: 94. 95. Múppes: Myrrhis: Finocchiella:

Lib. VII, cap. 28: Cicutaria.

Caulis fructifer (95), cum folio sejuncto (94); hoc ad Cicutariam tertiam Cæs. l. c. spectat, quæ Myrrhis odorata Scop., caulis ad Cicutariam alteram Cæs. vel Chcrophyllum magellense Ten!

38, 96. Siler aquaticum:

Lib. VII, cap, 26: Silaus.

Specimen floriferum. Oenanthe Phellandrium Lam.

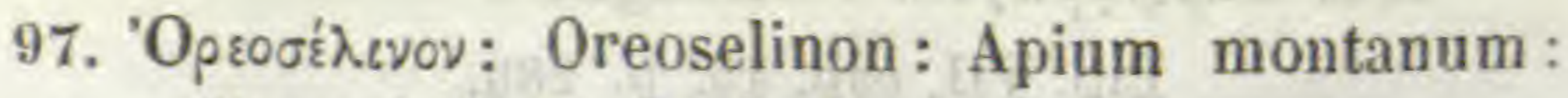
Chærefolium: Cerfoglo:

Lib. VII, cap. 44.

Specimen floriferum. Anthriscus Cerefolium Hoffm. 


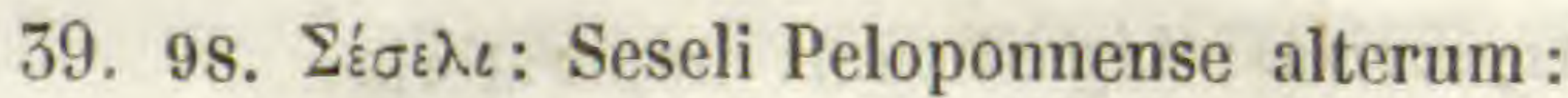

Lib. VII, cap. 33: Seseli Creticum alterum? Folium. Peucedanum venetum Koch.

99. Apium sylvestre :

Lib. VII, cap. 33: Seseli Creticum.

Specimen floriferum. Peucedanum Oreoselinum Moench.

40. 100. Пupéfoov: Pyrethrum: Pilatro:

Lib. VII, cap. 30.

Specimen floriferum. Peucedanum palustre Moench.

101. Topoú̀ıov: Tordylium: Seseli creticum: Pimpinella Romana:

Lib. VII, cap. 58.

Specimen flores et fructus ferens. Tordylium apulum Linn.

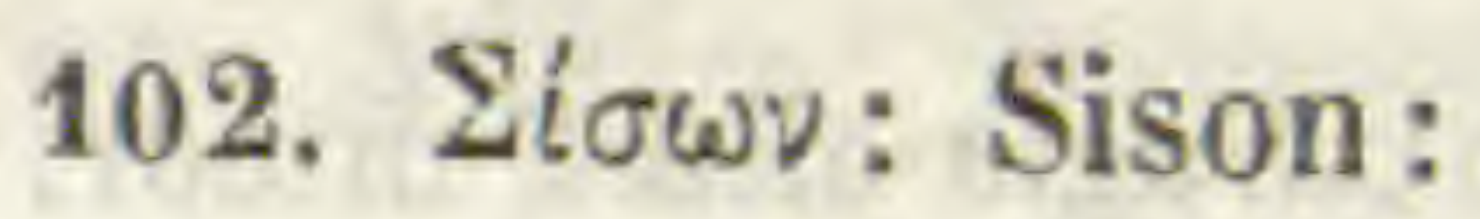

Lib. VII, cap. 40.

Specimen præcedenti simile. Sison Amomum Linn.

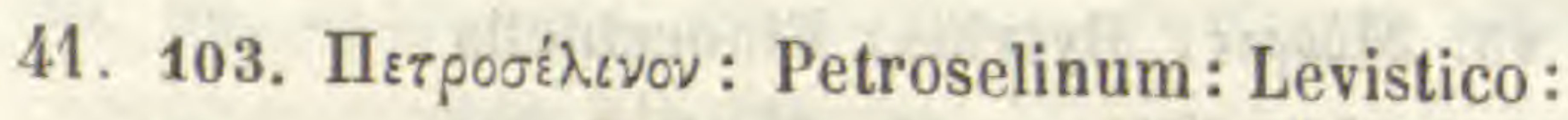

Lib. VII, cap. 45.

Specimen juvene, mox florendum. Peucedanum Cervaria Cuss.

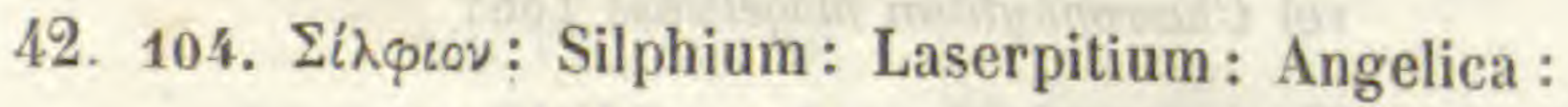
Lib. VII, cap. 48.

Folium. Angelica sylvestris Linn.

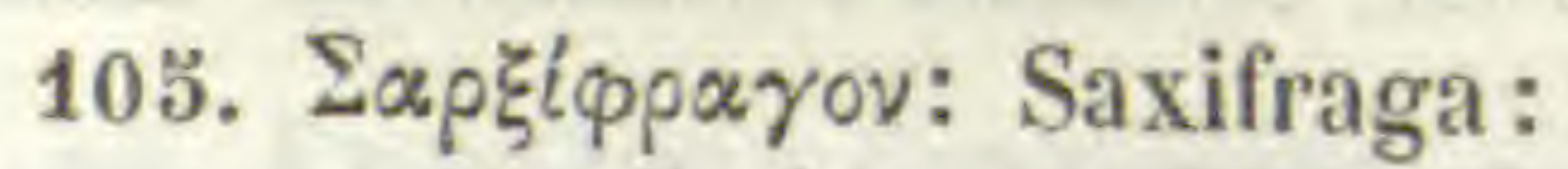

Lib. VII, cap. 15, p. 286.

Folia tria. Trinia vulgaris var. Dec.

Folia hujus varietatis Trinice vulgaris a foliis Seselis montani ægre distinguenda, saltem in siccâ plantâ. 


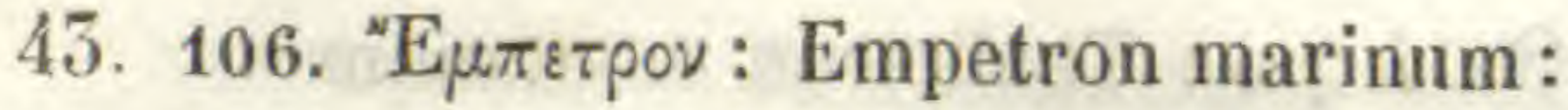

Lib. VII, cap. 36: Baticulæ alterum genus. Specimen floriferum. Seseli Bocconi Guss.

107. Crithmum sylvestre:

Lib. XIII, cap. 31 .

Specimen floriferum. Echinophora spinosa Linn.

44. 108 . Kpitrov: Crithmum: Batis. Pli: Baciuco:

Lib. VII, cap. 36: Baticula.

Prorsus fere deficit. Crithmum maritimum Linn.

109. "Е $\mu \pi \varepsilon \tau$ роv: Empetron alterum: Fendesasso:

Folium. Fateor me non invenire potuisse, cui plantæ pertinet.

45. 110. Bd่xx apts: Baccharis:

Lib. IV, cap. 5: Valeriana inodora prima.

Specimen fructiferum. Valeriana tripteris Linn.

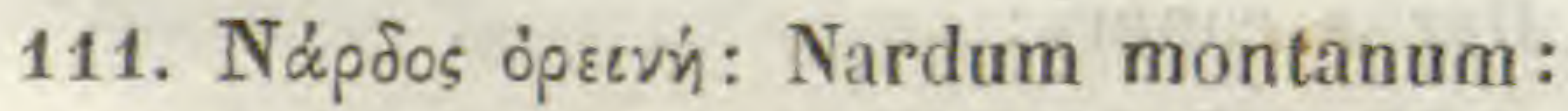

Folium, ut videtur Valerianæ cujusdam, sed mihi non licet speciem determinare. Nardum montanum in lib. IV, cap. 3 est verisimiliter Valeriana tuberosa Linn.

112. Saponaria :

Lib. IV, cap. 5: Valeriana inodora tertia.

Specimen fructiferum. Centranthus ruber Dec.

46. 113. Kарлиंльоv: Carpesium: Valeriana optima :

Lib. IV, cap. 2.

Specimen fructiferum. Valeriana Phu Linn.

114. Фоข̃ : Phu: Valeriana:

Lib. IV, cap. 2: Valeriana altera sylvestris.

Specimen floriferum. Valeriana officinalis Linn. 


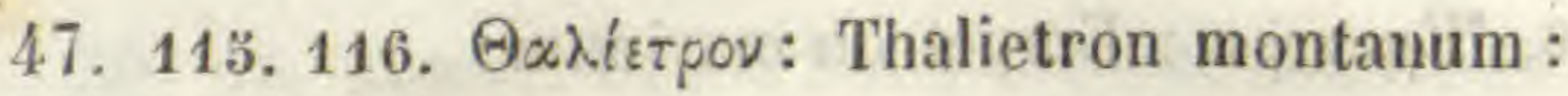

Lib. IX, cap. 2.

Panicula florifera (415) Rutæe sylvestris Cæs. I. c. vel Thalictri aquilegifolii Linn., cum folio sejuncto (146) ad Rutam sylvestrem alteram Cæs. 1. c. referendo, quæ est Thalictrum minus Linn. vel species affinis.

48. 117. 'I $\sigma \dot{\alpha} \iota_{5}$ : Isatis : Glastum sativum: Guado:

Lib. VIII, cap. 60.

Inflorescentiæ duæ, altera florifera altera fructifera. Isatis tinctoria Linn., varietas ad Reichenb. Ic. n. 4177 respondens.

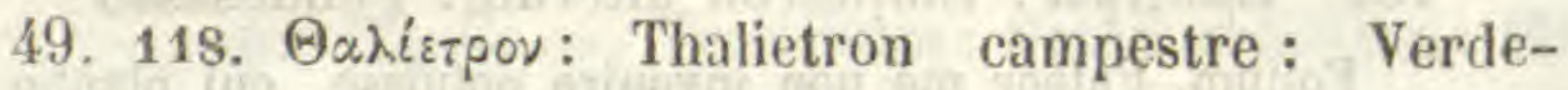
marco:

Lib. IX, cap. 3.

Specimen floriferum. Thalictrum angustifolium Jacq; Kach. Syn. ed. $2^{a}$.

50. 119. 'Нخєотро́ Herba cancri :

Lib. XI, cap. 11.

Specimen floriferum et fructiferum. Heliotropium europoeum Linn.

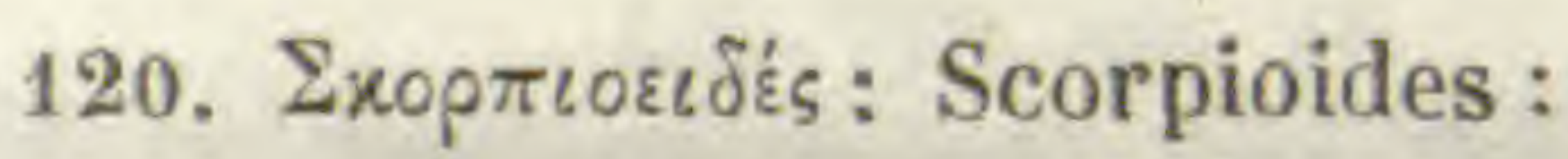

Lib. XI, cap. 12: Heliotropium minns, primum. Specimen floriferum. Myosotis palustris With; Koch Syn. ed. $2^{a}$.

121. Cynoglossa. Pli: Lingua canina:

Lib. XI, cap. 13: Cynoglossa prima.

Specimen floriferum. Cynoglossum pictum Ait.

51. 122. Calla 2a. Pli: Pulmonaria:

Lib. XI, cap. 5.

Specimen floriferum, cum folio radicali adjuncto. Pulmonaria tuberosa Schrank; Reichenb. Ic. VI. fig. 697. 
123. Cerinthe. Pli: Pulmonaria romana:

Lib. XI, cap. 6.

Specimen floriferum. Cerinthe aspera Roth.

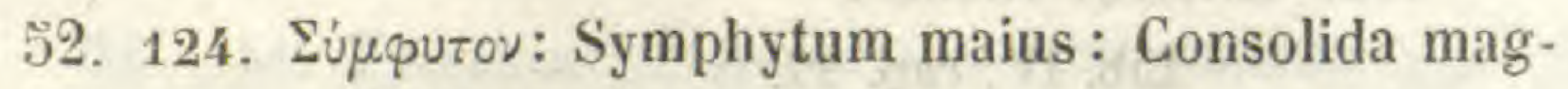
giore:

Lib. XI, cap. 7: Consolida prima.

Specimen floriferum. Symphytum officinale Linn.

53. 125. "A $\gamma \chi \circ \cup \sigma \alpha$ : Anchusa $p^{a}$ :

Lib. XI, cap. 9 : Echion.

Specimen floriferum. Echium italicum Linn.

126. "A $\gamma \chi \circ \cup \sigma \alpha$ : Anchusa 2:

Lib. XI, cap. 8: Anchusa maior.

Specimen fructiferum cum folio radicali adjuncto. Echium plantagineum Linn.

127. "A $\gamma \chi \circ \cup \sigma \alpha$ : Anchusa $3^{\mathrm{a}}$ :

Lib. XI, cap. 8 : Anchusa tertia.

Specimen flores et fructus ferens. Lithospermum arvense Linn.

54. 128. $\Lambda$ úxoభıs: Lycopsis : Buglossa hispana:

Lib. XI, cap. 4.

Specimen floriferum. Anchusa (Caryolopha) sempervirens Linn.

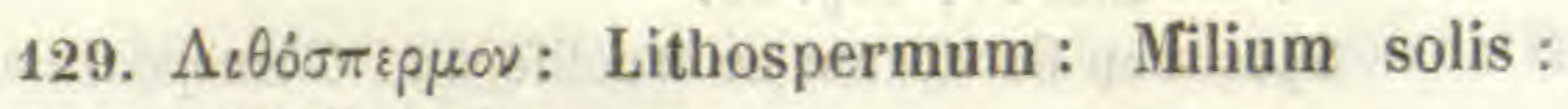
Miglialsole:

Lib. XI, cap. 8 : Anchusæ tertiæ similis altera? Specimen flores et fructus ferens. Lithospermum officinale Linn.

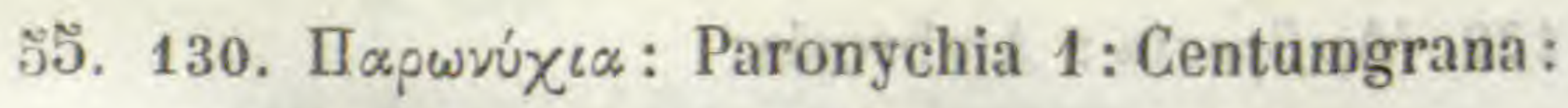

Lib. VI, cap. 54.

Specimen floriferum et fructiferum. Polyearpon tetraphyllum Linn. 
131. Paronychia altera:

Specimen ut antecedens. Scleranthus annuus. Linn.

132. 'A

\section{Lib. IV, cap. 37.}

Specimen fructiferum. Theligonum Cynocrambe Linn.

56. 133. По入ú yovov: Polygonum marinum:

Lib. IV, cap. 36: Polygon. aliud in marittimis. Specimen flores et fructus ferens. Polygonum maritimum Linn.

134. Пoגúrovov: Polygonum mas: Cenfinodia: Correggiola:

Lib. IV, cap. 36.

Specimen ut præcedens. Polygonum aviculare Linn.

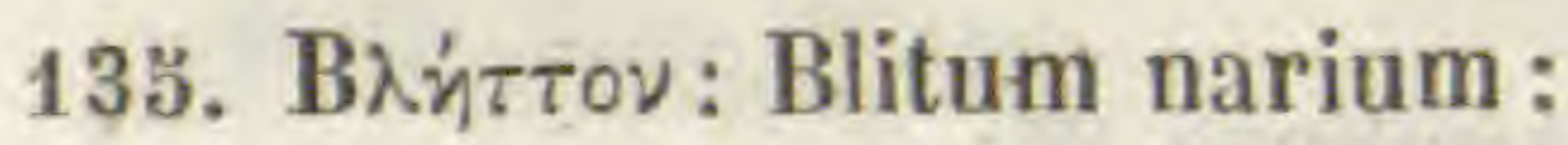

Lib. IV, cap. 26: Bliti peregrini alterum genus. Specimen fructiferum. Amarantus tricolor Linn.

57. 136. 'I $\sigma \alpha \tau \iota \varsigma$ : Isatis sylvestris:

Lib. VIII. cap. 60 : Herba Sancti Antonij.

Specimen nondum floridum. Plumbago europaa Linn.

137. Bótpus: Botrys:

Lib. IV, cap. 19.

Specimen floriferum ac fructiferum. Chenopodium Botrys Linn.

138. Ambrosia altera :

Cauliculus florifer. Corrigiola litoralis Linn.

139. 'A $\mu$ ßporia: Ambrosia:

Lib. IV, cap. 20: Herba Turca montana?

Specimen juvene. Herniarioe hirsutce Linn. varietas glabrescens. 
140. Ambrosia altera:

Lib. IV, cap. 20 : Herba Turca.

Ramulus. Altera varietas Herniarice hirsutce Linn.

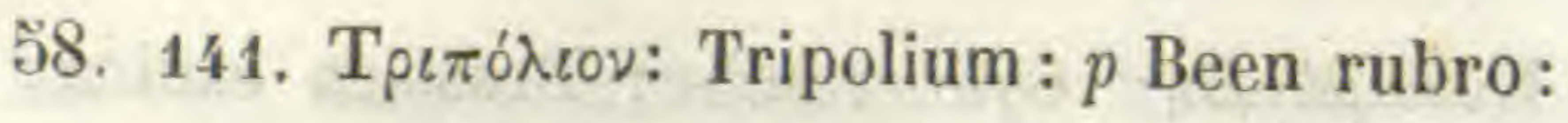

Lib. IV, cap. 32 : Limonium.

Folium. Statice Limonium Linn. Sp; Bert. Fl. ital.

142. Tripolium parvum:

Lib. IV, cap. 32: Limonii genus exiguum.

Specimen nondum floridum, ad Staticem virgatam Willd., Boiss. in Dec. Prodr. XII, p. 654 (S. olecefoliam Bert. FI. ital. III, p. 516) ut videtur referendum.

143. Lapathum montanum :

Lib. IV, cap. 29: Lapathum in montibus quoddam.

Folium. Rumex alpinus Linn.

144. Bistorta :

Lib. IV, cap. 33.

Folium. Polygonum Bistorta Linn.

ร9. 145. 'I $\pi \pi 0 \lambda \dot{\alpha} \pi \alpha \theta 0 \nu$ : Hippolapathum :

Lib. IV, cap. 29.

Folium. Rumex.....

146. $\Lambda \alpha \pi \alpha \theta_{0 \nu}$ : Lapathum sylvestre: Rumex: Romice :

Lib. IV, cap. 29.

Specimen fructiferum, fructibus immaturis. Rumew obtusifolius Linn; Mert. Koch Deutschl. Fl. II, p. 610 .

147. 'O $\dot{\alpha}_{\alpha \lambda \iota s}$ : Oxalis : Acetosa:

Lib. IV, cap. 30 .

Folium. Rumex Acetosa Linn. Sp. 
148. Oxalis altera:

Lib. IV, cap. 30 : 0xalis sponte nascens. Specimen floriferum, mas. Rumex Acetosella Linn.

60. 149. Kuvoxp $\alpha \mu \beta \eta$ : Cynocrambe: Brassica canina: Connina :

Lib. IV, cap. 25.

Specimen flores et fructus ferens. Chenopodium Vulvaria Linn.

150. 'A $\tau^{\alpha} \alpha \alpha_{\xi} \iota s:$ Atraphaxis: Atriplex sylvestris :

Pes auserinus:

Lib. IV, cap. 25: Cynocrambe alterum.

Specimen ut præcedens. Chenopodium murale Linn.

151. Atriplex sylvestris altera :

Lib. IV, cap. 23.

Specimen floriferum. Chenopodium ficifolium Smith.

61. 152. Halimus:

Lib. IV, cap. 24: Halimum secundum. Specimen sterile. Atriplex Halimus Linn.

153. "A $\lambda \iota \mu$ s: Halimus :

Lib. IV, cap. 24 : Halimum primum.

Specimen fructiferum. Obione portulacoides Moq.

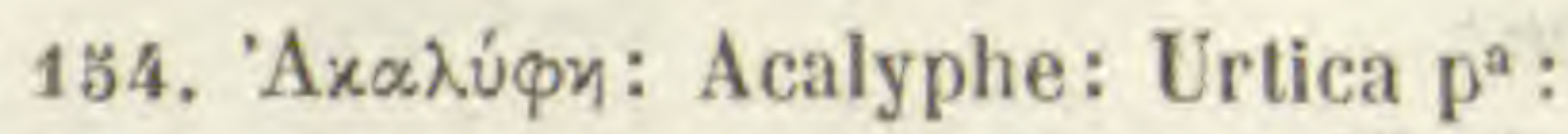

Lib. IV, cap. 15.

Specimen floriferum. Urtica pilulifera Linn.

62. 155. Formentone:

Lib. IV, cap. 31 .

Specimen floriferum cum fructibus nonnullis. Fagopyrum emarginatum Meisn. in Dec. Prodr. XIV, p. 143. 
156. Formentone salvatico:

Lib. IV, cap. 31 .

Specimen floriferum et fructiferum. Polygonum Convolvulus Linn.

63. 157. Lupus salictarius. Pli: Lupulo:

Lib. IV, cap. 17.

Panicula florifera mascula, cum folio. Humulus Lupulus Linn.

158. K $\alpha v v \alpha \beta \iota s$ : Cannabis sativa: Canapa:

Lib. IV, cap. 16.

Specimen floriferum masculum. Cannabis sativa Linn.

64. 159. Persicaria:

Lib. IV, cap. 34: Persicaria folio asperiusculo sine macula.

Specimen floriferum et fructiferum. Polygonum lapathifolium Linn ; Smith FI. brit. I, p. 425.

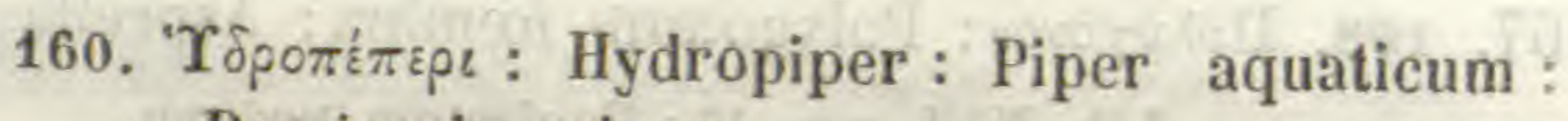
Persicaria minor:

Lib. IV, cap. 3 .

Specimen sterile. An Polygonum serrulatum Lag?

6อ̃. 161. Pes Leonis : Alchimilla maior:

Lib. XIV, cap. 24.

Specimen floriferum et fructiferum. Alchemilla vulgaris Linn.

162. Alchimilla minor:

Lib. XIV, cap. 24: Alchimillæ alterum genus.

Specimen fructiferum. Alchemilla alpina Linn.

163. $\Lambda \varepsilon \iota \chi \dot{y} \nu:$ Lichen: Pulmonaria:

Lib. XVI, cap. 20.

Thallus sterilis. Stiota pulmonaria Ach. 
66. 164. Chamæcyparissos. Pli:

Lib. XVI, cap. 21 : Muscus.

Specimen sterile. Hypnum alopecurum Linn.

165. Chamsecyparissos:

Lib. XVI, cap. 22: Musci genus quoddam folia Cupressi imitans?

Specimen sterile. Selaginella denticulata Koch.

166. Chamæcyparissos:

Lib. XVI, cap. 21 : Muscus.

Specimina mascula fertilia. Polytrichum commune Linn?

167. Chamæeyparissos :

Lib. XVI, cap. 22 : Musci genus quod pro Spica Celtica vendebatur.

Specimen fertile. Lycopodium annotinum Linn.

67. 168. Пlohúyovov: Polygonum fœemina: Asprella:

Lib. XVI, cap. 15: Equisetum tertium.

Caulis sterilis. Equisetum Telmateja Ehrh.

68. 169. ${ }^{\circ} \mathrm{I} \pi \pi$ oup di cavallo : Asprella:

Lib. XVI, cap. 15: Equisetum primum.

Caulis sterilis, Equisetum ramosum Schleich.

170. 'I $\pi$ roupls: Hippuris: Equisetum minus: Coda di cavallo: Asprella :

Lib. XVI, cap. 15: Equisetum secundum. Caulis sterilis. Equisetum arvense Linn.

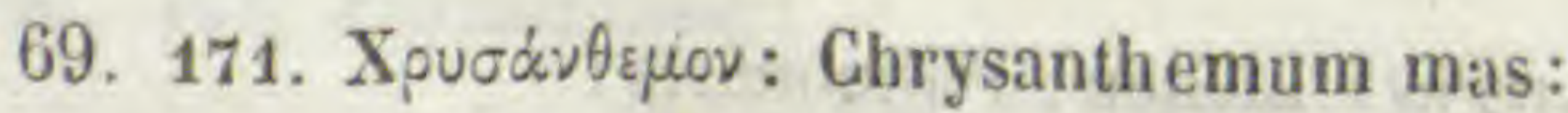

Lib. XII, cap. 28: Chrisanthemum ex Sicilia? Specimen floriferum. Chrysanthemum coronarium Linn. 


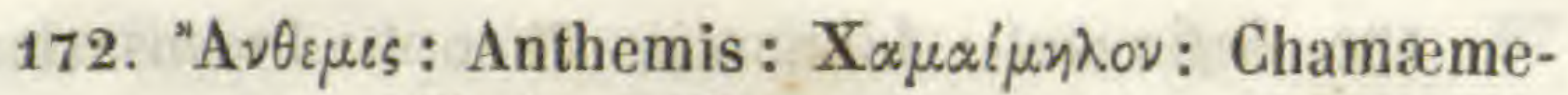
lum : Chamomilla :

Lib. XII, cap. 22.

Specimen mancum, capitulis omnino fere tarmete destructis. Matricaria Chamomilla Linn.

173. Mapดéviov: Parthenium: Gauta: Cotula foetida: Lib. XII, cap. 23.

Specimen fructiferum. Anthemis Cota Linn; Bert. Fl. ital. IX, p. 357.

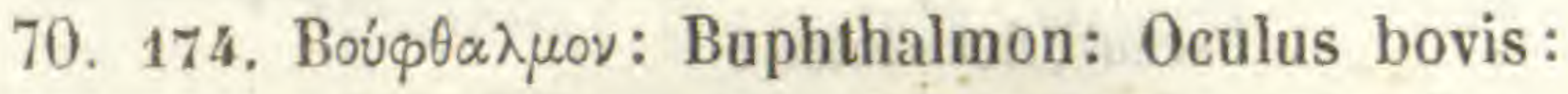

Lib. XII, cap. 27: Chrisanthemum?

Specimen floriferum. Anacyclus radiatus Lois.

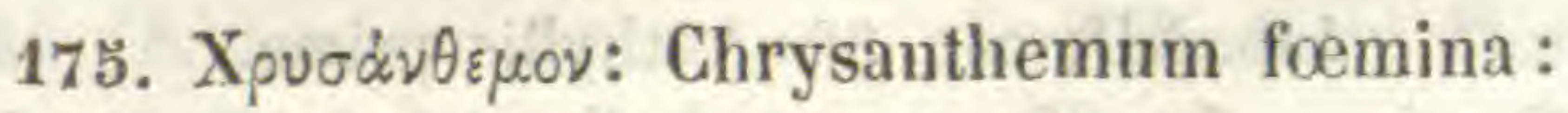

Lib. XII, cap. 28: Chrysanth. primum in arvis. Specimen floriferum et fructiferum. Chrysanthemum segetum Linn.

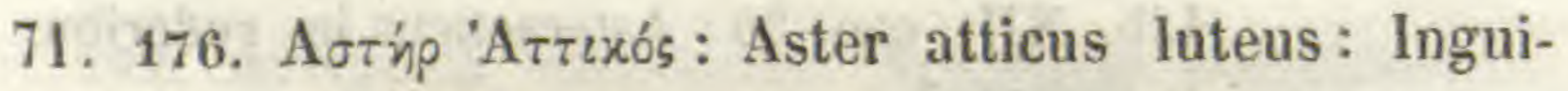
nalis:

Lib. XII, cap. 30: Asteracticus primus.

Specimen fructiferum. Asteriscus spinosus Gren. Godr.

177. 'Èㄹvov: Helenium: Enula campana: Ella :

Lib. XII, cap. $3 \%$.

Specimen floriferum. Inula Helenium Linn.

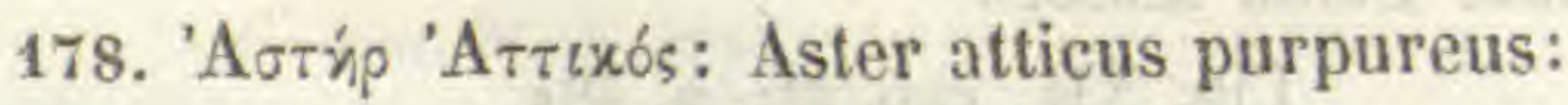

Lib. XII, cap. 30 : Asteracticus in alpibus nascens, flore cœruleo.

Specimen floriferum. Aster alpinus Linn.

179. Doronicum:

Lib. XII, cap. 33.

Folium Doronici cujusdam. 


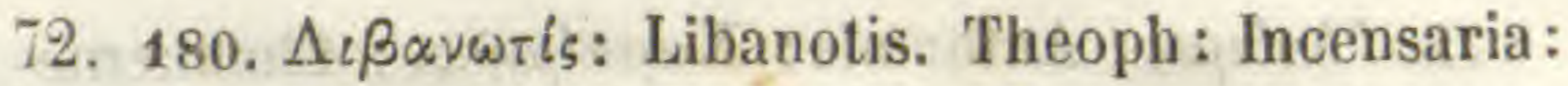

Lib. XII, cap: 34.

Specimen flores el fructus ferens. Pulicaria odora Reichenb.

181. Incensaria minor:

Lib. XII, cap. 32: Conyzá altera.

Specimen ut præcedens. Pulicaria dysenterica Gærtn.

182. Aster atticus alter:

Lib. XII, cap. 30: Asteracticus in alpibus nascens, flore luteo?

Specimen floriferum. Inula hirta Linn.

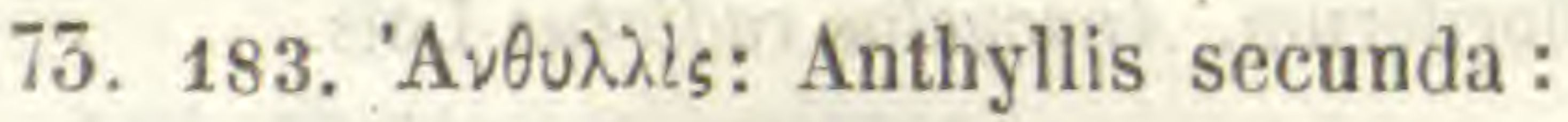

Lib. XII, cap. 38: Anthyllis prima.

Specimen fructiferum, capitulis autem vacuis. Inula crithmoides Linn.

184. Aster atticus alter:

Lib. XII, cap. 30 : Asteracticus in ruderibus nascens?

Specimen floriferum. Inula spirceifolia Linn. Sp. ed. 2a, p. 1238.

185. Птарцєхи́: Ptarmica altera :

Lib. XII, cap. 25.

Specimen sterile. Achillea Ptarmica Linn.

74. 186. Bellis maior:

Lib. XII, cap. 26 , p. 494.

Specimen ut videtur fructiferum, sed capitulo fructibus destituto. Leucanthemum vulgare Lam.

187. Bellis maior luteus:

Lib. XII, cap. 28: Chrisanthemum in arvis nascens, secundum.

Specimen ut præcedens. Chrysanthemum Myconis Linn. 


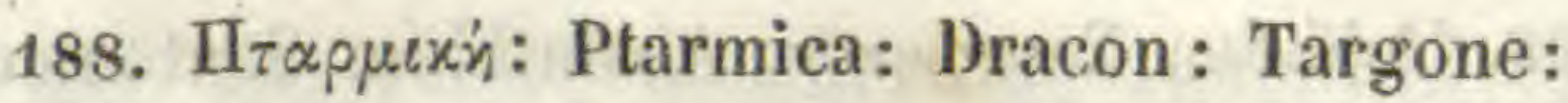

Lib. XIV, cap. 36.

Specimen floriferum. Artemisia Dracunculus Linn.

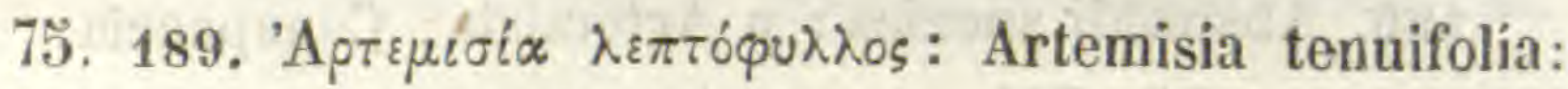
Matricaria :

Lib. XII, cap. 24.

Specimen floriferum. Pyrethrum Parthenium Smith.

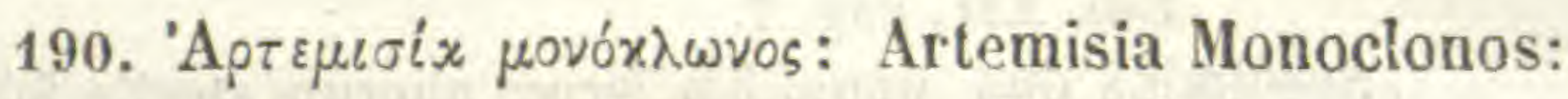
Athanasia: Tanacetum: Daneda :

Lib. XII, cap. 8.

Specimen floriferum. Tanacetum vulgare Linn.

76. 191. 'A $\gamma$ ńpatov: Ageraton: pro Eupatorio Mesues: Canforata :

Lib. XII, cap. 9.

Specimen floriferum. Achillea Ageratum Linn.

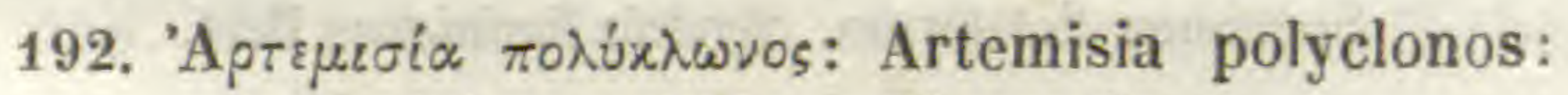
Artemisia maritina: Cineraria:

Lib. XII, cap. 36.

Specimen floriferum. Senecio Cineraria Dec.

193. Herba. S. Jacobi :

Lil. XII, cap. 37: Herba Sancti Jacobi foliis ad Raphani folia magis accedentibus (secundum Mich. mss.).

Specimen floriferum. Senecio erraticus Bert.

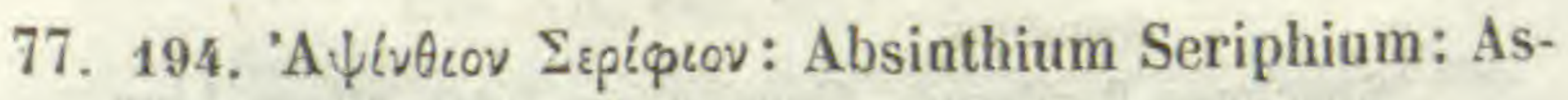
senzo marino:

Lib. XII, cap. 2: Absinthii alterum genus. Specimen sterile, ut duarum sequentium stirpium. Artemisia ccerulescens Linn? 


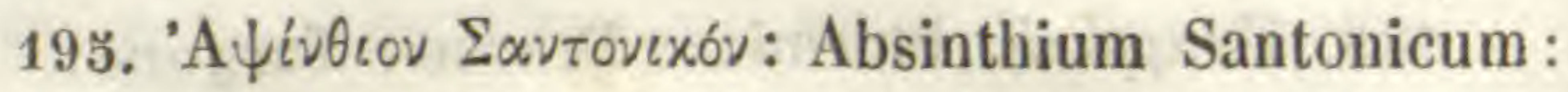

Lib. XII, cap. 4.

Artemisia pontica Linn.

196. Absinthii species : $p$ Artemisia : Canapaccia:

Lib. XII, cap. 5: Artemisia vulgaris.

Artemisia vulgaris Linn.

78. 197. 'A ßpótovov: Abrotonum foemina : Santolina :

Lib. XII, cap. 7.

Specimina duo, unum floriferum alterum sterile foliiferum. Santolina Chamceyparissus Linn; varietates duæ.

198. Aßpotovov: Abrotonum mas:

Lib. XII, cap. 6.

Specimen sterile. Artemisia camphorata Vill.

79. 199. ' $H \rho \prec \gamma(\rho \omega \nu$ : Erigeron: Senecio:

Lib. XIII, cap. 51 : Senecio maior.

Specimen floriferum. Senecio laciniatus Bert.

200. Impia:

Lib. XII, cap. 15.

Specimen floriferum. Filago germanica Linn.

201. Impia minor:

Lib. XII, cap. 15: Herba Impia alia.

Specimen floriferum. Filago gallica Linn.

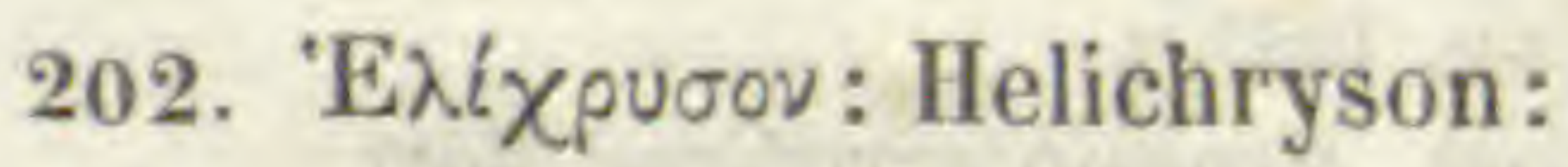

Lib. XII, cap. 14: Chrysocome in plana descendens.

Specimen floriferum. Gnaphalium luteo-album Linn.

203. Xpuбoxópn: Chrysocome:

Lib. XII, cap. 14: Chrysocome in petris nascens.

Specimen floriferum. Phagnalon sordidum Dec. 
80. 204. 'E^ıxpuøov: Helichryson: Timiamica: Tignamica :

Lib. XII, cap. 13.

Specimen floriferum. Helichrysum angustifolium Dec.

205. Chrysocome alterum:

Lib. XII, cap. 14: Chrysocome in montibus. Specimen floriferum. Gnaphalium sylvaticum Linn; G. rectum Smith.

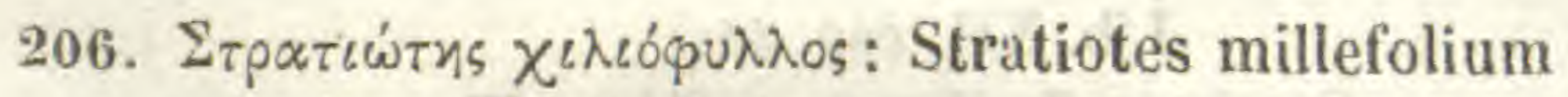
maius: Millefoglo:

Lib. XII, cap. 11.

Specimen floriferum. Achillea Millefolium Linn.

207. Stratiotes millefolium minus: Millefoglo:

Lib. XII, cap. 11.

Specimen floriferum. Speciei præcedentis varietas minor.

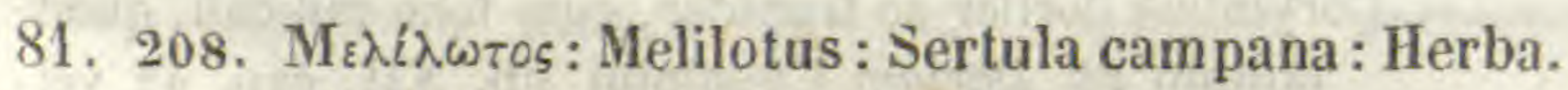
S. Maria.

\section{Lib. XII, cap. 12.}

Specimen nondum floridum. Tanacetum Balsamita Linn.

209. Bì $\prec$ เov: Bechinm: Tussilago: Farfara :

Lib. XII, cap. 21.

Folium Tussilaginis Farfarce Linn.

82. 210. Kaxa入ia: Cacaiia:

Lib. XII, cap. 19.

Specimen floriferum. Adenostyles alpina Bluff. Fing.

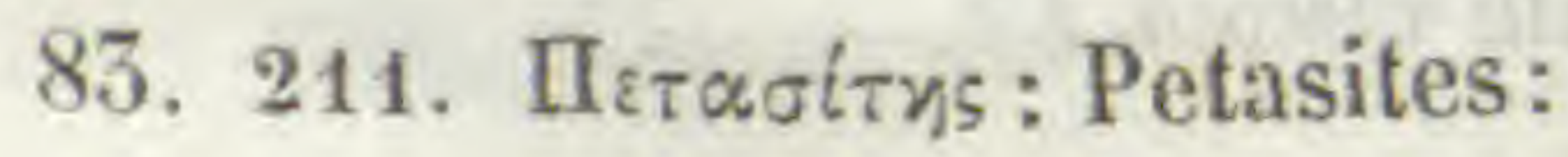

Lib. XII, cap. 20.

Folium Pelasitis officinalis Mœnch. 
84. 212. "Apxtıov: Arctium:

Lib. XII, cap. 18.

Specimen floriferum. Inula Conyza Dec.

213. Eupatorio affinis folio dissecto: (1)

Lib. XII, cap. 17 : Bidens foliis tripartito divisis. Specimen sterile, Bidens tripartita Linn.

85. 214. Pharnaceon. Pli. tenuioris folii : Virga aurea Campestris :

Lib. XII, cap. 44.

Specimen floriferum. Solidago Virgaurea Linn.

215. Eupatorium Avicennæ:

Lib. XII, cap. 16.

Specimen vix floridum. Eupatorium cannabinum Linn.

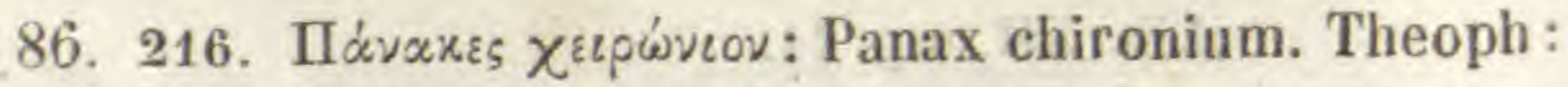
Doria :

Corymbus florifer ac fructifer, cum folio radicali, quod tantum ad Doriam Cæsalpini lib. XII, cap. 42 vel Senecionem Doriam Linn, pertinet, dum ille alienæ plantæ nempe Asteris Tripolii Linn, verisimiliter boc loco per errorem positi, inflorescentiam constituit.

217. Pharnaceon levioris folii, Pli: Virga aurea montana:

Lib. XII, cap. 41: Virgæ aureæ alterum genus? Specimen floriferum. Senecio saracenicus Linn?; Koch Syn. ed. ${ }_{2 a}$; Reichenb. Iconogr. cent. 3, fig. 468.

87. 218. Kóvǔ̌ $\alpha$ : Conyza maior : Pulicaria :

Lib. XII, cap. 40.

Specimen sterile. Inula viscosa Ait.

(1) Nomen aliena manu scriptum, quod et hie et in indice Cresalpinus emiserat. 
219. Kóvงఢ̧ $\alpha$ : Conyza minor:

Lib. XII, cap. 40.

Specimen floriferum. Inula graveolens Desf.

220. Conyza purpurea :

Specimen floriferum. Erigeron acre Linn.

221. Conyza parva:

Specimen floriferum. Linosyris vulgaris Cass.

88. 222. Pilosella :

Rosula foliorum radicalium Hieracii Pilosellce Linn. (quod vera Pilosella Cæs. lib. XIII, cap. 18), cum scapo Hieracii piloselloides Vill.

223. 'Iepáxıov : Hieracium parvum:

Lib. XIII, cap. 21: Lactuca montana?

Specimen nondum floridum. Prenanthes purpurea Linn.

224. ¿óxхos: Sonchus levis: Cicerbita:

Lib. XIII, cap. 11, p. 512: Sonchus in collibus umbrosis?

Specimen ut præcedens. Lactuca muralis Fres.

89. 223. 'A $\alpha \dot{\alpha}$ : : Apate. Theoph: Fraus:

- Micheli mss. et Bertoloni Fl, ital. ad Condrillam aliam lib. XIII, cap. 7 referunt.

Specimen fertile. Zacintha verrucosa Gærtn.

226. Ginestrella:

Lib. XIII, cap. 16.

Specimen floriferum. Thrincia hirta Roth. 


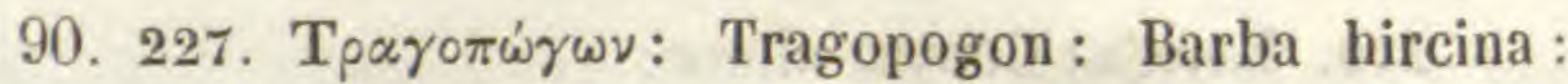
Sassefrica :

Lib. XIII, cap. 23.

Specimen floriferum, capitulo omnino fere tarmete destructo. Tragopogon pratense Linn.

228. П เxpis : Picris. Theoph : Amarago:

Lib. XIII, cap. 8.

Specimen floriferum. Urospermum Dalechampii Desf.

229. Lattaiola :

Lib. XIII, cap. 22?

Specimen floriferum et fructiferum. Hieracium prenanthoides Vill. var. (H. elatum Fries?).

91. 230. $\Theta p i \delta \alpha \xi \grave{\alpha} \gamma \rho i \alpha$ : Tridax agria: Lactuca sylvestris: Scariola :

Lib. XIII, cap. 20.

Specimen floriferum. Lactuca Scariola Linn.

231. Xov $\rho i \lambda \lambda \eta$ : Chondrilla $p^{2}$ :

Lib. XIII, cap. 6.

Specimen floriferum, valde mancum. Chondrilla juncea Linn.

232. Endivia sylvestris : (1)

Lib. XIII, cap. 22: Lactariola alia in arvis.

Specimen floriferum. Helminthia echioides Gærtn.

92. 233. ₹6́xхos: Sonchus aspera:

Specimen fertile. Urospermum picroides Desf.

234. Crepis. Pli : Terracrepolo:

Lib. XIII, cap. 14.

Specimen fructiferum. Picridium vulgare Desf.

(1) Nomen e manu aliena, ut in $\mathbf{n}^{0} 213$; in indice etiam deest. 


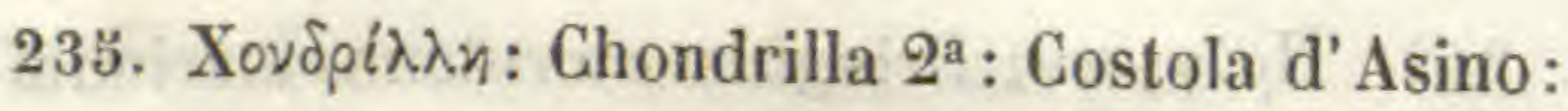

Lib. XIII, cap. 5 .

Specimen fertile, sed valde mancum, rosula foliorum radicalium tantum exstante. Ut videtur est Hypoc hoeris radicata Linn.

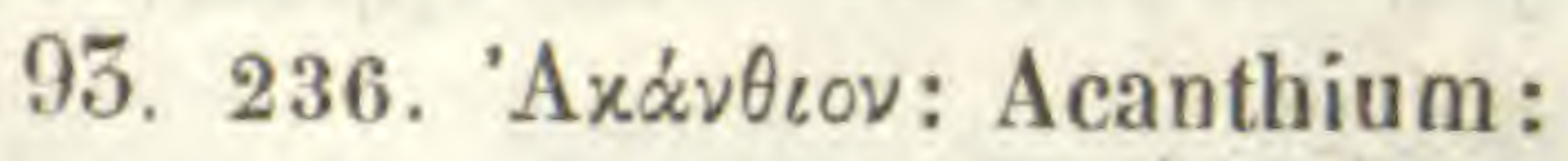

Lib. XIII, cap. 36: Carduus sylvestris.

Specimen floriferum. Onopordum Acanthium Linn.

94. 237. "Axopva: Acorna. Theoph:

Lib. XIII, cap. 45.

Specimen floriferum. Carlina corymbosa Linn.

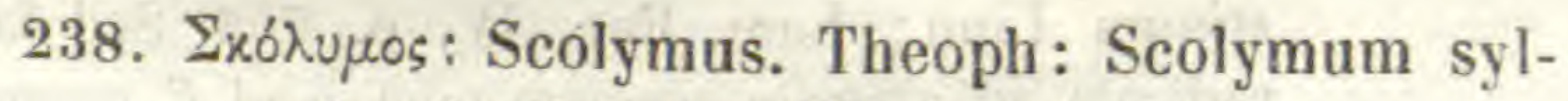
vestre fruticosum. Pli :

Lib. XIII, cap. 33.

Caulis florifer, cum folio radicali. Scolymus hispanicus Linn.

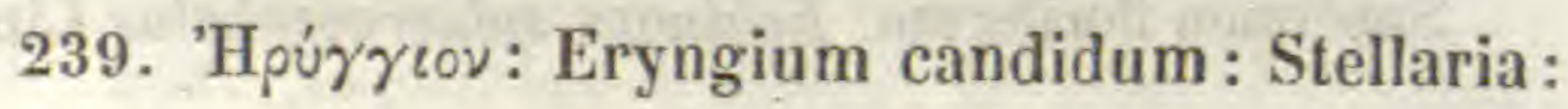

Lib. XIII, cap. 49: Cacatreppola altera.

Specimen fructiferum. Centaurea solstitialis Linn.

95. 240. $\Delta$ íqaxos: Dipsacus: Labrum veneris: Scardaccione:

Lib. XIII, cap. 27.

Caulis florifer, cum reliquiis folii radicalis. Dipsacus sylvestris Mill.

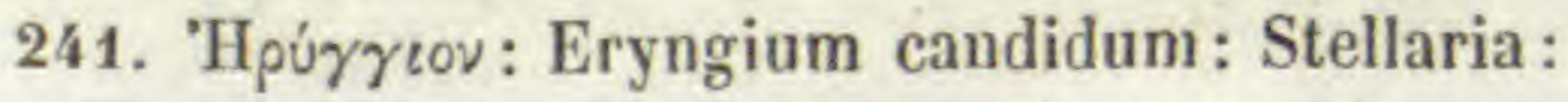

Lib. XIII, cap. 48 : Cacatreppola. Specimen fructiferum, mancum. Centaurea Calcitrapa Linn. 


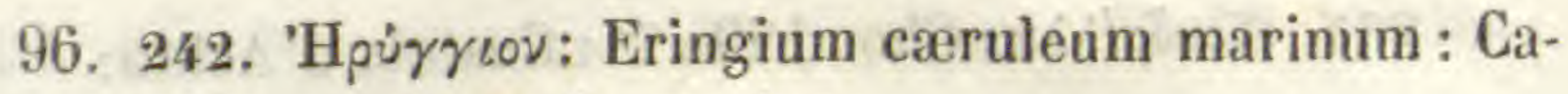
catreppola marina:

Lib. XIII, cap. 29.

Specimen floriferum. Eryngium maritimum Linn.

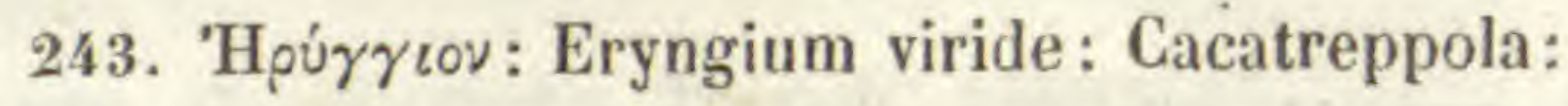

Lib. XIII, cap. 28.

Caulis florifer, cum rosula foliorum radicalium. Eryngium campéstre Linn.

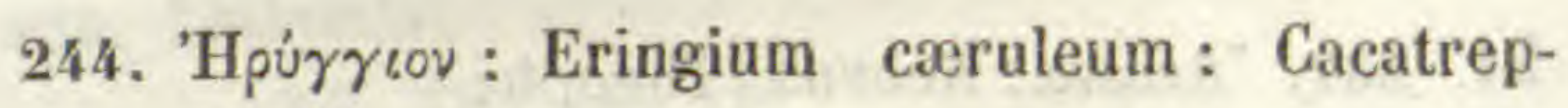
pola :

Lib. XIII, cap. 28: Eryngium aliud in montibus. Caulis florifer, cum folio radicali. Eryngium-amethystinum Linn; Lam. Enc. IV, p. 702. Sed folium radicale ad alteram speciem nempe ad $\boldsymbol{E}$. campestre pertinet.

97. 245. Centumcapita. Pli :

Lib. XIII, cap. 30: Agriacantha?

Specimen floriferum. Echinops spharocephalus Linn.

98. 246. Kvixos: Cnicus sátivus: Cartamus: Gruogo saracinesco:

\section{Lib. XIII, cap. 46.}

Specimen fructiferum. Carthamus tinctorius Linn.

247. Kvíxos: Cnicus sylvestris 2s. Theoph: Atractylis purpurea. Diosc :

\section{Lib. X1II , cap. 47 , p. 535.}

Specimen floriferum. Centaurea sphcerocephala Linn.

248. 'A

Lib. XIII, cap. 4h: Carthamum sylvestre. Specimen floriferum. Kentrophyllum lanatum Dec. 
99. 249. Kpiosıov: Cirsium montanum:

Lib. XIII, cap. 43 : Cirsium secundum.

Specimen floriferum. Carduus Personata Jacq.

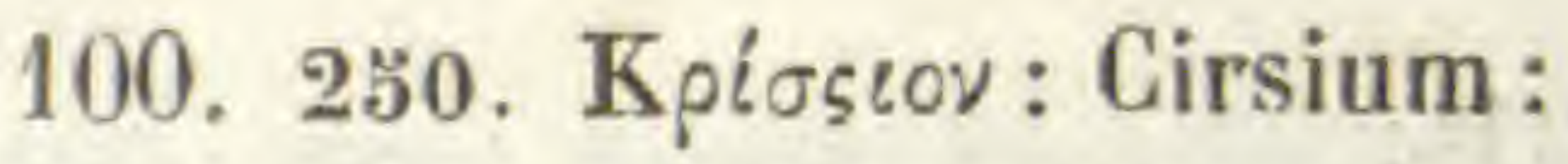

Lib. XIII, cap. 43: Cirsium primum.

Specimen fertile; sed pars inferior foliifera tantum superest. Cirsium monspessulanum All.

251. X $\alpha \mu \alpha \iota \lambda_{\varepsilon}^{\prime} \omega \nu$ ó $\lambda \varepsilon v$ oós: Chamæleon albus: Carlina bianca:

Lib. XIII, cap. 38.

Folium. Carlina acanthifolia All.

252. Cyanus. Pli: Fioraliso grande:

Lib. XIII, cap. 52: Cyanus secundus.

Specimen valde mancum, e frustulo acephalo cum foliis duobus radicalibus constans. Centaurea nigrescens? vel alia ejusdem sectionis.

101. 253. Х $\alpha \mu \alpha \iota \lambda_{\varepsilon}^{\prime} \omega \nu$ o $\mu \varepsilon^{\prime} \lambda \alpha_{5}$ : Chamæleon niger: Carlina nera:

Lib. XIII, cap. 39: Carlinæ alterum genus, Folium. Carlina acaulis Linn.

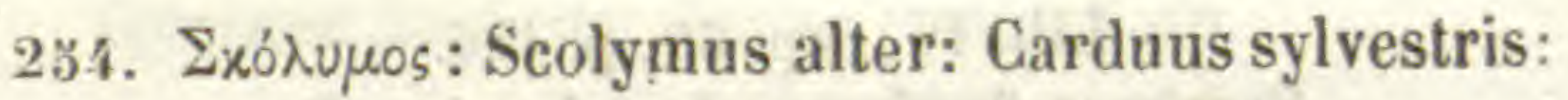

Lib. XIII, cap. 41 : Spina Arabica?

Specimen floriferum. Carduus nutans Linn.

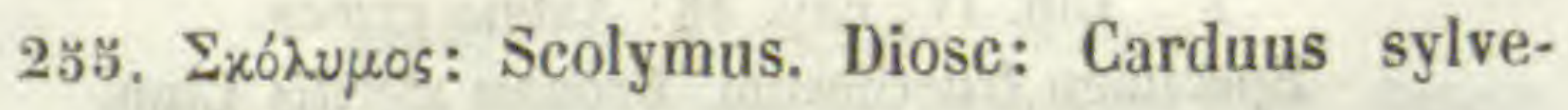
stris :

- Lib. XIII, cap. 40: Spina alba alia?

Specimen valde mancum, cum folio et reliquiis floris tantum exstantibus. Cirsium lanceolatum Scop. 
102. 256. Cyanus sylvestris:

Lib. XIII, cap. 54 .

Specimen floriferum. Xeranthemum cylindraceum Sibth. Sm.

257. Cyanus sylvestris:

Lib. XIII, cap. 55: Papaver spumeum aliud?

Specimen fertile. Centaurea alba Linn.

258. „Apxєเov: Arcion : Personacea : Bardana: Lappa maggiore:

Lib. XIII, cap. 50.

Specimen floriferum. Lappa minor Dec.

103. 259.

Lib. IV, cap. 58.

Specimen floriferum. Agrostis vulgaris With.

260.

Lib. IV, cap. 58.

Specimen floriferum. Poa trivialis Linn.

261.

Lib. IV, cap. 58.

Specimen floriferum. Briza media Linn.

262. Ischæmon. Pli : Herba Lucciola:

Lib. IV, cap. 58, p. 486.

Specimen floriferum. Luzula nivea Dec.

104. 263. Egylops: 'A

Lib. IV, cap. 48: Avena græca.

Specimen fructiferum. Bromus sterilis Linn.

264. Ara. Pli :

Lib. IV, cap. 58, p. 486 : Herba capillaris?

Specimen floriferum. Deschampsia flexuosa Trin. 
265. Bromos. Pli :

Lib. IV, cap. 58, p. 186: Perlara.

Specimen floriferum. Arrhenatherum avenaceum Rœm. Schult.

105. 266. $\Phi$ oivv

Lib. IV, cap. 58 , p. 185.

Specimen fructiferum. Scleropoa rigida Gris.

267. Tpádos: Tragos:

Lib. IV, cap. 58.

Specimen floriferum. Serrafalcus mollis Parl,

268. Ordeum Murinum. Pli:

Lib. IV, cap. 58 , p. 185.

Specimen floriferum. Hordeum murinum Linn.

269. Lolium sylvestre:

Lib. IV, cap. 58, p. 185.

Specimen floriferum. Lolium italicum Al. Braun?

106. 270. "A $\gamma$ pwstıs: Agrostis: Gramen harundinaceum:

Lib. IV, cap. 58, p. 185: Planta in ruderibus culmo inclinato, foliis candida lanugine obsitis etc.

Specimen floriferum. Holcus lanatus Linn.

271. Stelephuros. Pli : Cauda ramosa:

Lib. IV, cap. 58, p. 185: Herba spica molli et lanuginosa, racemulis distincta?

Specimen floriferum. Trisetum neglectum Rœm. Schult.

272. Stelephuros, Pli : Cauda ramosa:

Specimen floriferum. Iterum Trisetum neglectum. 
273. Alopecuros. Pli : Cauda vulpina:

Lib. IV, cap. 58, p. 184.

Specimen fere destructum, sed a reliquiis absque dubio Lagurus ovatus Linn.

107. 274. "Arpwsıs: Agrostis': Gramen aculeatum. Theoph: Sanguinella:

Lib. IV, cap. 57: Gramen.

Specimen fertile, Cynodon Dactylon Pers.

275. $\Phi \dot{d} \lambda \lambda$ apts : Phalaris :

Specimen fructiferum. Panicum Crus galli Linn.

108. 276. Juncus parvus:

Lib. IV, cap. 60.

Specimen floriferum, Carex remota Linn.

277.

Lib. IV, cap. 58.

Specimen fructiferum. Eragrostis megastachya Link.

278. Panicum sylvestre:

Lib. IV, cap. 58, p. 185.

Specimen fructiferum. Phleum asperum Jacq.

109. 279. Cyperus hortensis:?

Lib. IV, cap. 63: Trasi.

Specimen sterile. Cyperus esculentus Linn.

280. Juncus parvus 2:

Lib. IV, cap. 60.

Specimen fructiferum. Carex extensa Good.

281. Juncus parvus 3 :

Lib. IV, cap. 60.

Specimen floriferum. Juncus lamprocarpus Ehrh. 
110. 282. II $\dot{\alpha} \pi$ upos : Papyrus:

Lib. IV, cap. 65.

Umbella. Cyperus syriacus Parl.

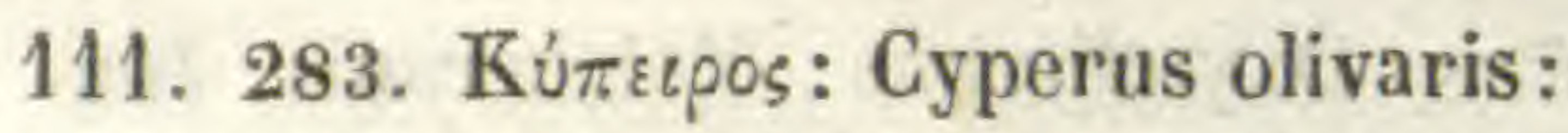

Lib. IV, cap. 61: Cyperus secundus.

Specimen floriferum. Cyperus rotundus Linn?; C. olivaris Targ!

284. Kúmeıpos: Cyperus :

Lib. IV, cap. 61: Cyperus primus.

Specimen floriferum. Cyperus longus Linn.

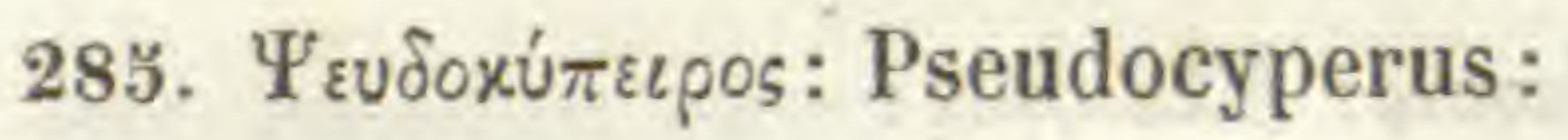

Lib. IV, cap. 64.

Specimen fructiferum. Scirpus maritimus Linn.

112. 286. Túøx: Typha: Schianza:

Lib. IV, cap. 68.

Specimen mancum, spicâ destructâ, folio tantum exstante. Typha latifolia vel potius $T$. angustifolia Linn.

\section{Giunchi : (1)}

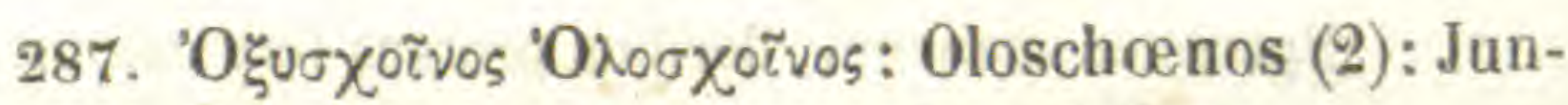
cus acutus:

Lib. IV, cap. 60 : Junci secundum genus? Specimen fructiferum. Juncus acutus Linn.

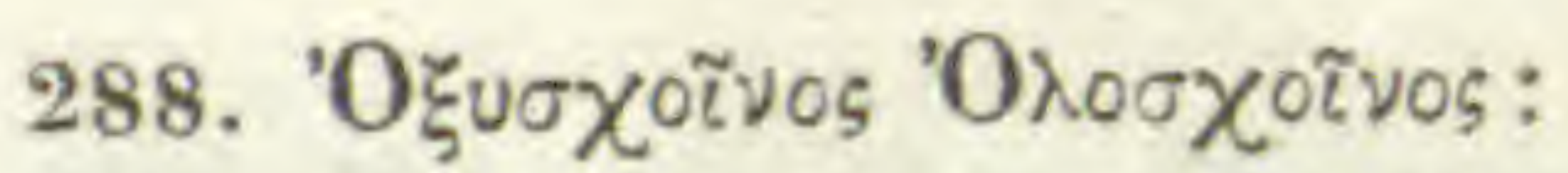

Lib. IV, cap. 60.

Specimen fructiferum. Juncus glaucus Ehrh.

(1) Sub hoc nomine complectuntur Ni, 287-291.

(2) Oloscheni nomen inter hoc et specimen sequens ambigit. 


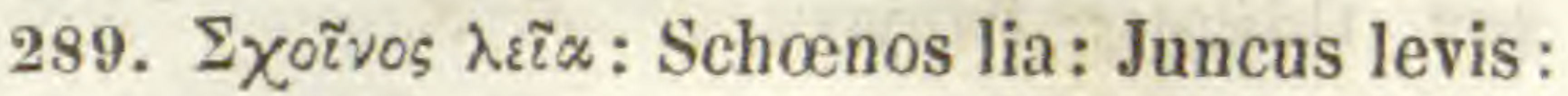

Lib. IV, cap. 60 : Junci primum genus?

Specimen fructiferum. Soirpus Holoschœenus Linn.

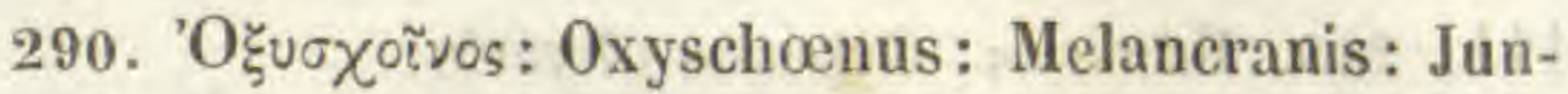
cus acutus:

Lib. IV, cap. 60: Junci tertium genus.

Specimen fructiferum. Scirpus lacustris Linn.

291. Juncus parvus:

Lib. IV, cap. 66: Phalaris prima (secundum Micheli mss.).

Specimen fertile. Carex muricata Linn.

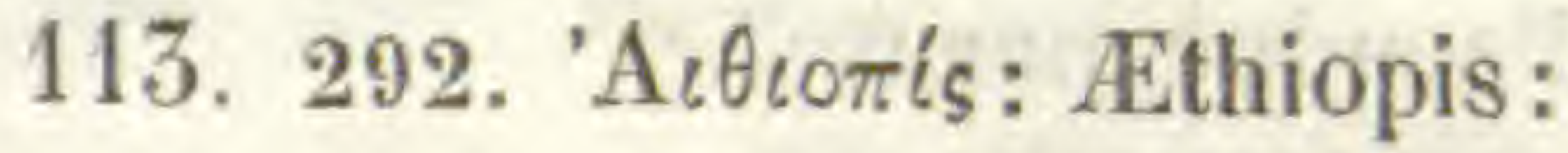

Lib. XI, cap. 49.

Racemus florifer, cum folio radicali. Salvia Ethiopis Linn.

293. Alectorolophos. Pli: Crista Gallinacea : Chiarella minore :

Lib. XI, cap. 18: Gallitricum.

Specimen floriferum. Salvia Verbenaca Linn.

114. 294. Scopa regia. Pli: Sclarea: Chiarella maggiore: Lib. XI, cap. 17.

Specimen mancum, floribus prorsus fere carens. Salvia Sclarea Linn.

295. Scopa regia. Pli: Sclarea : Chiarella maggiore:

Racemus florifer et fructifer, cum folio. Salvia virgata Ait.

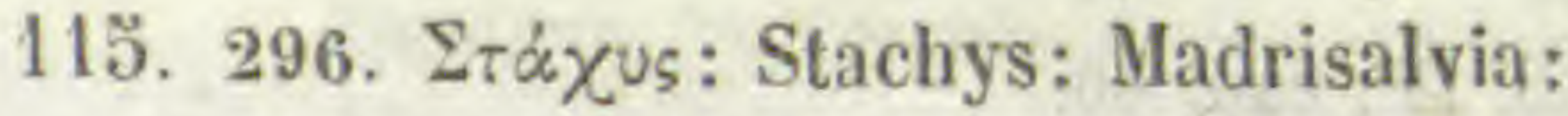

Lib. XI, cap. 16: Salvia sylvestris prima candida.

Specimen floriferum, floribus autem tarmete erosis carens. Stachys germanica Linn. 
297. "O $\rho \mu \iota$ ○оv: Horminum sylvestre:

$$
\text { Lib. XI, cap } 29 .
$$

Specimen floriferum. Melittis Melissophyllum Linn.

116. 29s. Betonica. Pli : Melino:

$$
\text { Lib. XI, cap. } 20 .
$$

Specimen sterile. Salvia glutinosa Linn.

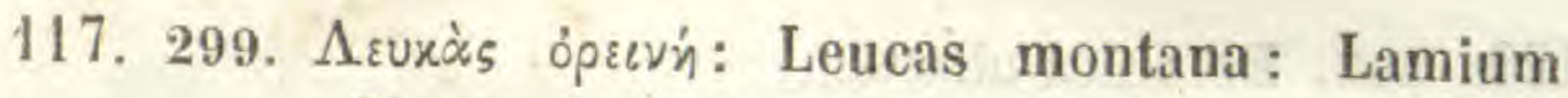
Montanum:

$$
\text { Lib. XI, cap. 31, p. } 450 .
$$

Specimen floriferum. Lamium Galeobdolon Crantz.

300. Betonica altera :

Lib. XI, cap. 21.

Specimen floriferum. Stachys sylvatica Linn.

118. 301. Tertiola :

Lib. XI, cap. 34.

Specimen floriferum. Stachys palustris Linn.

302. $\Delta v \sigma \iota \mu \alpha x^{i o v}:$ Lysimachia purpurea:

Lib, VI, cap. 55.

Specimen floriferum et fructiferum. Lythrum Salicaria Linn.

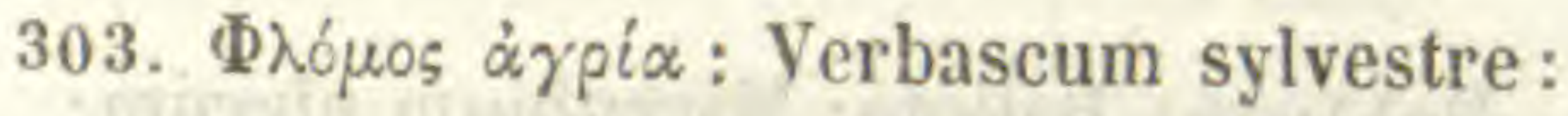

Lib. XI, cap. 28.

Specimen floriferum. Phlomis fruticosa Linn.

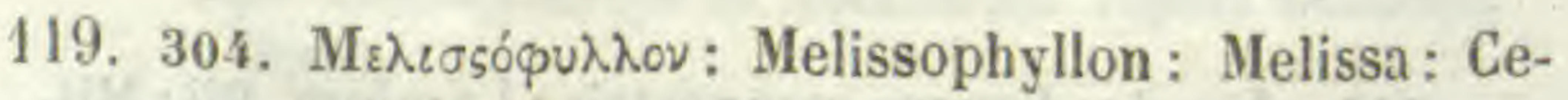
dornella :

Lib. XI, cap. 25.

Specimen floriferum et fructiferum. Melissa officinalis Linn. 
30з. K'́spov: Cestron: Betonica:

Lib. XI, cap. 21.

Specimen floriferum. Betonica officinalis Linn.

306. Myriophyllon alterum:

Lib. VI, cap. 60: M. alterum.

Specimen fertile. Myriophyllum verticillatum Linn.

120. 307. Betonica altera:

Lib. XI, cap. 20 : Melinum alterum.

Specimen floriferum. Teucrium Scorodonia Linn.

308. Пєрє૬єрєஸ่ข: Peristereon: Verbenaca recta :

Lib. XI, cap. 33.

Specimen floriferum. Lycopus europceus Linn.

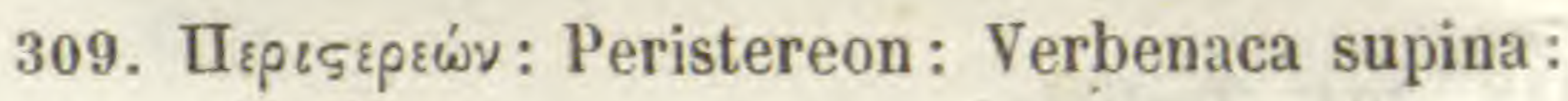
Verbena:

Lib. XI, cap. 32.

Specimen floriferum. Verbena officinalis Linn.

121. 310. Tparopíravos: Tragoriganum maius:

Lib. XI, cap. 37: Herba Origano vulgari similis etc.

Specimen floriferum. Calamintha Clinopodium Benth.

311. B $\alpha \lambda \lambda \omega \tau \dot{\text { : }}$ : Ballote: Marrubium nigrum:

Lib. XI, cap. 22.

Specimen floriferum. Ballota fretida Lam.

312. $\Lambda$ zuxd́s: Leucas campestris: Lamium. Pli : Milzadella:

Lib. XI, cap, 31.

Specimen floriferum. Lamium maculatum Linn. 
122. 313. Пpágiov: Prasium: Marrubium: Marrobbio: Lib. XI, cap. 26.

Specimen floriferum. Marrubium vulgare Linn.

\section{Cardiaca:}

Lib. XI, cap. 23.

Specimen floriferum et fructiferum. Leonurus Cardiaca Linn.

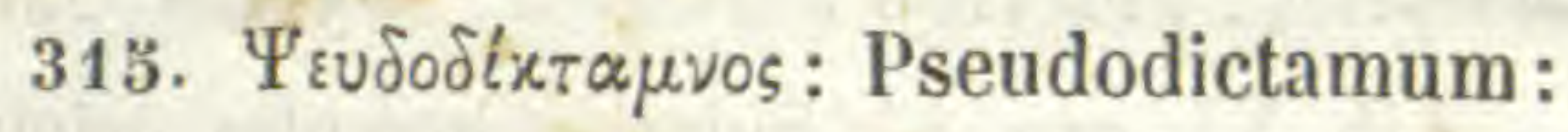

Lib. XI, cap. 27 : Planta Cretensis pro Pseudodictamo allata.

Specimen floriferum. Ballota Pseudodictamnus Benth.

123. 316. Prunella altera :

Lib. XI, cap. 37: Prunella secunda.

Specimen floriferum. Brunella alba Pall., Koch.

317. Morella: Prunella : Herba mora:

Lib. XI, cap. 37: Prunella prima.

Specimen floriferum. Brunella vulgaris Linn., Koch, integrifolia.

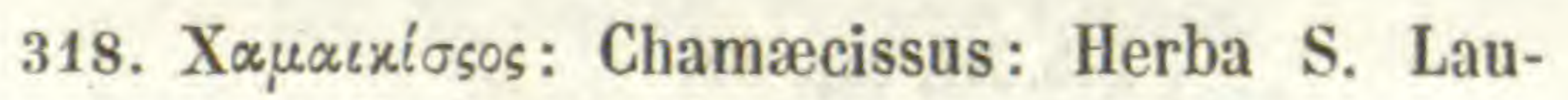
rentii: Laurentina:

Lib. XI, cap. 36 , p. 453.

Specimen floriferum. Ajuga reptans Linn.

319. Edera terrestris:

Lib. XI, cap. 36.

Specimen floriferum et fructiferum. Nepeta Glechoma Benth.

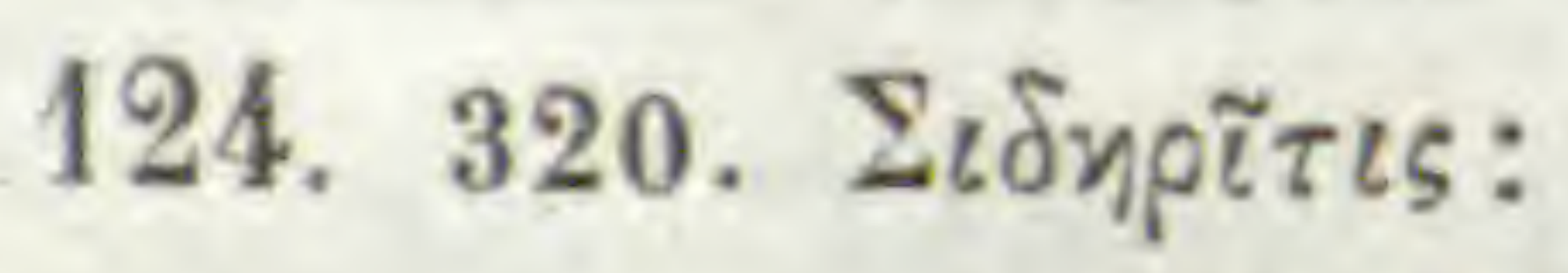

Lib. XI, cap. 35: Sid. in vineis, flore purpureo. Specimen floriferum. Galeopsis angustifolia Ehrh. 


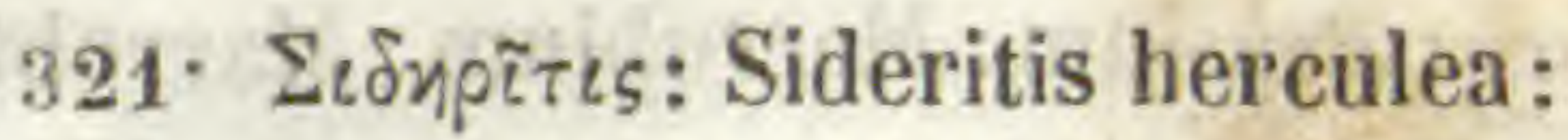

Lib. XI, cap. 35: Sideritis herculea secunda. Specimen floriferum. Stachys annua Linn.

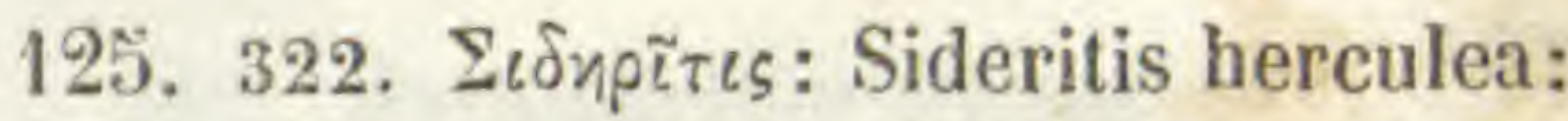

Lib. XI, cap. 35: Sid. in vineis, flore albicante. Specimen fructiferum. Stachys recta Linn.

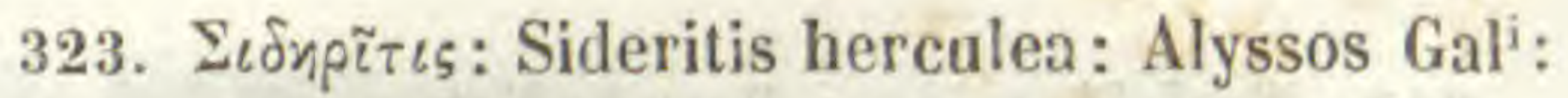

Lib. XI, cap. 35: Sideritis herculea prima. Specimen floriferum et fructiferum. Sideritis romana Linn.

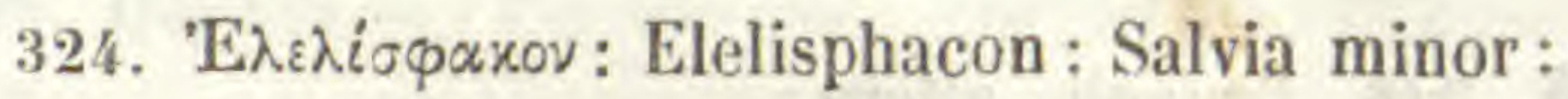
Lib. XI, cap. 15. Specimen floriferum. Salvia lavandulafolia Vahl.

325. Tparopiravos: Tragoriganum parvum:

Plane deficiens. Anne referendum ad Origanum alterum sylvestri serpillo simile lib. XI, cap. 52, p. 464 ?

126. 326. K $\alpha \lambda \alpha \mu$ ì $\theta$ : Calamentum montanum:

\section{Lib. XI, cap. 62:}

Specimen floriferum. Calamintha grandiflora Mœnch.

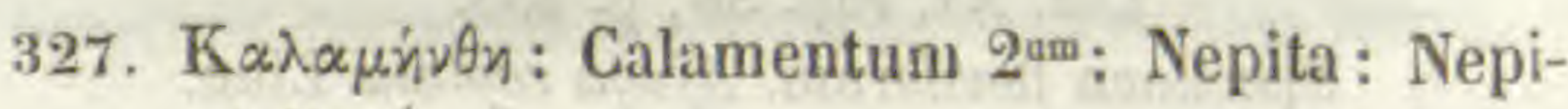
tella :

Lib. XI, cap. 61 .

Specimen floriferum. Calamintha officinalis Mœnch; var. $\beta$ Koch Deutschl. fl. IV, p. 319.

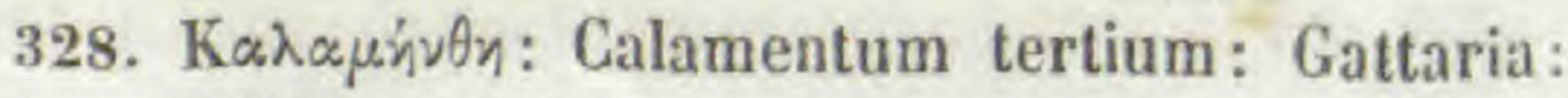
Herba gatta:

$$
\text { Lib. XI, cap. } 63 .
$$

Specimen floriferum. Nepeta Cataria Linn. 


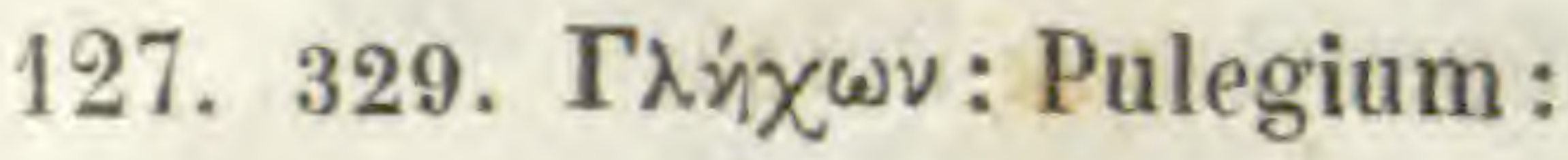

Lib. XI, cap. 60.

Specimen floriferum. Mentha Pulegium Linn.

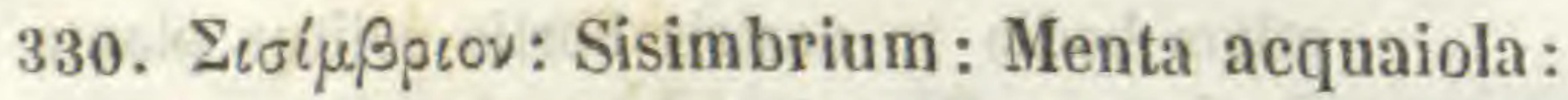

Lib. XI, cap. 64 : Menta sylvestris prima.

Specimen floriferum. Mentha aquatica Linn., Benth.

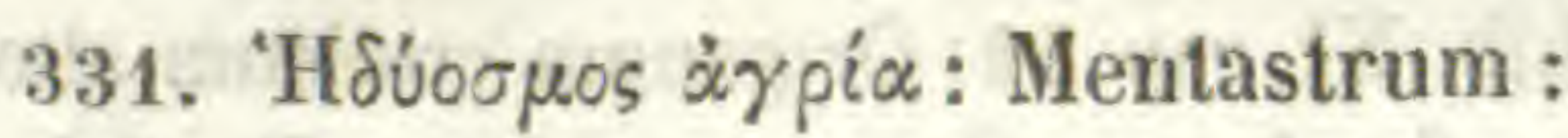

Lib. XI, cap: 64: Menta sylvestris secunda. Specimen floriferum. Mentha sylvestris Linn., Benth.

332. K $\iota \iota$ ขo $6 \delta \delta\llcorner 0 \nu$ : Clinopodium : $p$ Isopo montano:

Lib. XI, cap. 39.

Specimen floriferum. Micromeria juliana Benth.

333. 'E

Lib. XI, cap. 54 .

Specimen floriferum. Thymus Serpyllum Linn.

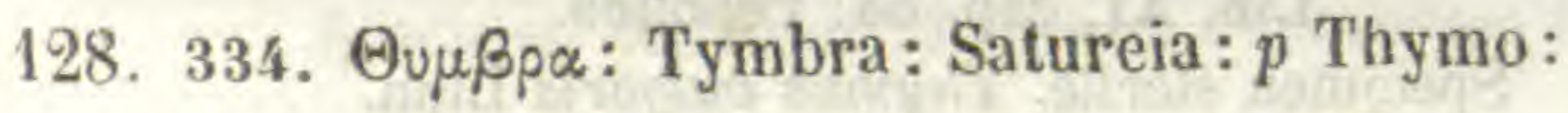

Lib. XI, cap. 48: Thymum Italicum.

Specimen floriferum. Satureia montana Linn.

335. Thymbra altera: $p$ Issopo domestico:

Lib. XI, cap. 50: Hyssopum.

Specimen floriferum. Hyssopus officinalis Linn.

336. Mápov: Marum: Maggiurana:

Lib. XI, cap. 57 : Maiorana.

Specimen floriferum. Origanum Majorana Linn.

337. $\Upsilon_{\sigma \varsigma \omega \pi 05}$ : Hyssopum creticum: $p$ Origano:

Lib. XI, cap. 51 : Origanum creticum. Inflorescentia tantum. Origanum Onites Linn. 
338. $\Sigma \dot{\alpha} \mu \psi v \chi 0 v:$ Sampsuchum: Persa:

Lib. XI, cap. 53: Scarsapepe.

Specimen sterile. Origanum creticum Savi, an Linn? Sub hoc saltem nomine Persa vel Persia nera etiam hodie in nostris hortis colitur planta quam Savius pro 0 . cretico determinavit.

339. 'Op'ravos: Origanum heracleoticum : Regamo:

Lib. XI, cap. 52: Origanum creticum alterum? Specimen floriferum. Origanum hirtum Benth!

340. 'Opiravos: Origanum Onitis : Regamo:

Lib. XI, cap. 52: Origanum Italicum. Specimen floriferum. Origanum vulgare Linn.

129. 341. $\Delta i x \tau \alpha \mu \nu$ os: Dictamum:

Lib. XI, cap. 58.

Specimen floriferum. Origanum Dictamnus Linn.

342. 'Е $\rho \pi \lambda_{\lambda}$ os: Serpyllum rectum:

Lib. XI, cap. 55: Pepolina.

Specimen sterile. Thymus vulgaris Linn.

343. Qúros: Thymum eretense:

Lib. XI, cap. 47.

Specimen sterile. Thymus capitatus Hoffm. et Link.

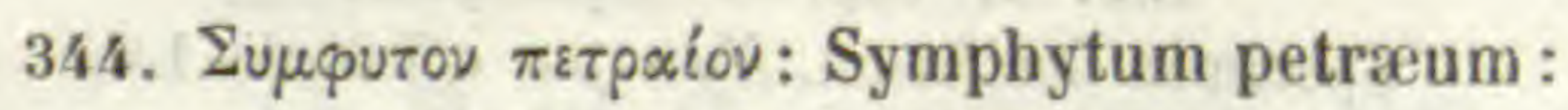

Lib. XI, cap. 44, p. 458: Polium tertium.

Specimen floriferum. Teucrium montanum Linn. angustifolium.

345. ПÍ́̉ıov: Polium montanum:

Lib. XI, cap. 44, p. 458: Polium secundum.

Specimen floriferum. Teucrium montanum Linn. latifolium. 


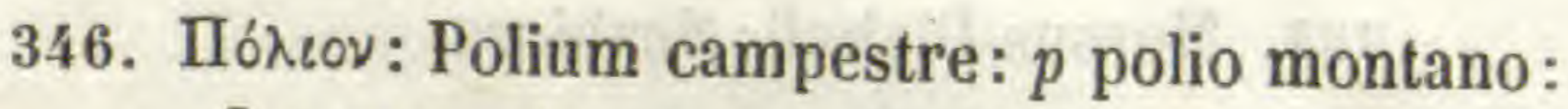
Iva moscata :

Lib. XI, cap. 44: Polium primum.

Specimen floriferum. Teucrium Polium Linn.

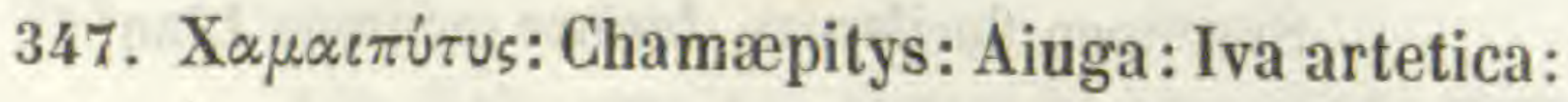

Lib. XI, cap. 43.

Specimen floriferum et fructiferum. Ajuga Chamapitys Schreb.

130. 348. $\Sigma_{\tau \circ \iota \chi} \alpha_{s}:$ Stœechas: Sticados:

Lib. XI, cap. 46: Spica Italica sylvestris.

Specimen nondum floridum. Lavandula Stoechas Linn.

349. Téxprov: Teucrium :

Lib. XI, cap. 41: Teucrium primum.

Specimen floriferum. Teucrium flavum Linn.

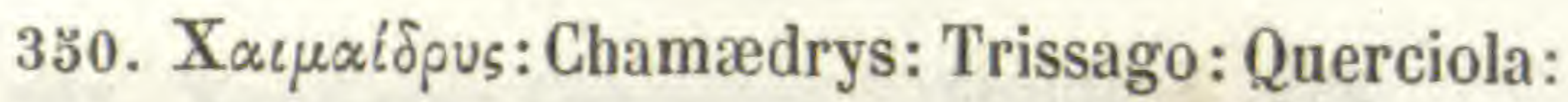

Lib. XI, cap. 40.

Specimen floriferum. Teucrium Chamcedrys Linn.

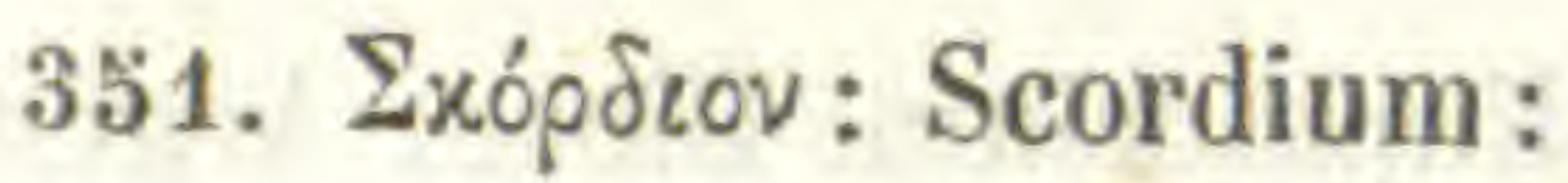

Lib. XI, cap. 42.

Specimen floriferum. Teucrium scordioides Schreb.

352. K $\alpha \sigma \varsigma \cup \propto \alpha$ : Cassutha : Cuscuta :

Lib. VIII, cap. 41.

Omnino fere deficiens. Probabiliter Cuscuta Epithymum.

131. 3ร3. Scabiosa:

Lib. XIII, cap. 57: Scab. foliis crassius divisis. Specimen nondum floridum. Cephalaria transylvanica Schrad. 
354. Morsus Diaboli: Scabiosa:

Lib. XIII, cap. 59 ,

Specimen ut præcedens. Scabiosa Succisa Linn.

355. Scabiosa:

Specimen floriferum. Jasione montana Linn.

356. Scabiosa :

Lib. XII, cap. 26, p. 494: Bellis montana. Specimen floriferum. Globularia incanescens Viv.

132, 357. Scabiosa:

Lib. XIII, cap. 60: Scabiosa arborescens.

Specimen floriferum. Scabiosa cretica Linn.

358. Scabiosa :

Specimen valde mancum, caussâ tarmetis. Anne Scabiosa columbaria?

359. Scabiosa:

Lib. XIII, cap. 57: Scab. foliis tenuius divisis. Specimen floriferum. Scabiosa maritima Linn.

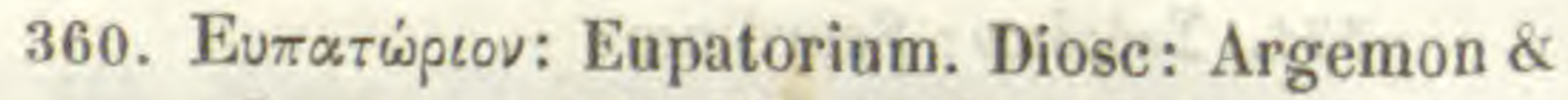
Lappa canaria. Pli; Agrimonia :

Lib. VIII, cap. 4.

Specimen floriferum. Agrimonia Eupatoria Linn.

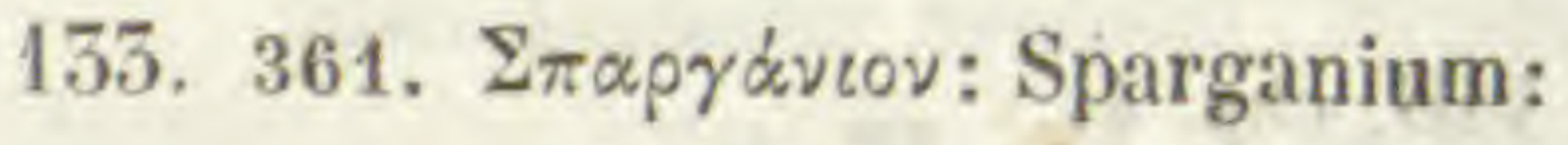

Lib. IV, cap. 67.

Specimen floriferum. Sparganium ramosum Huds.

362.

Lib. IV, cap. 13: Parvus frutex in Alpibus nascens similis Cassiæ ligneæ.

Specimen floriferum. Thesium alpinum Linn. 


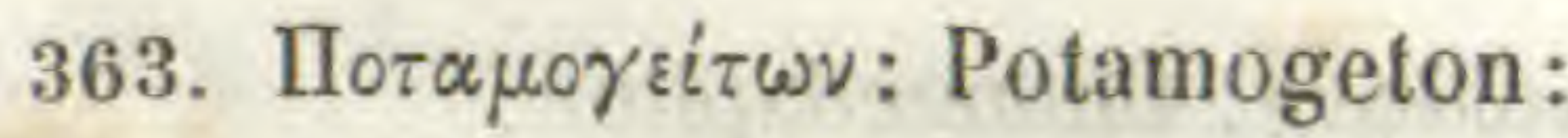

Lib. VIII, cap. 22.

Specimen fertile, sed spicà amissâ. Potamogeton natans Linn.

364. Scabiosa :

Lib. XIII, cap. 17: Genus in montibus, foliis ab radice tantum, veluti Graminis, cauliculo nudo, ac simplici, flore simili Scabiosæ.

Specimen floriferum, sed valde mancum. Armeria, an elongata?

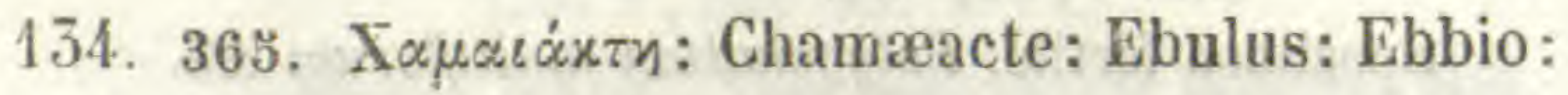

Lib. V, cap. 13.

Ramulus florifer cum folio. Sambucus Ebulus Linn.

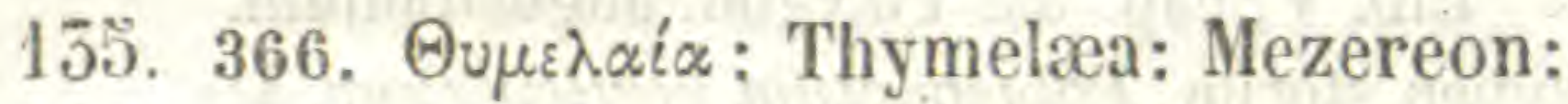

Lib. IV, cap. 9.

Specimen floriferum. Daphne Gnidium Linn.

367. X $\alpha \mu \varepsilon \lambda \alpha i \alpha$ : Chamelæa: Olivella:

Lib. IV, cap. 7: Chamelæa altera.

Specimen foliiferum. Daphne Mezereum Linn.

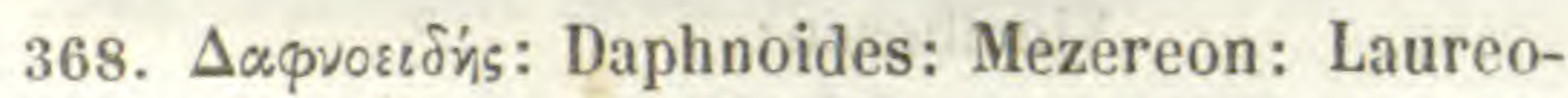
la: Oriola :

Lib. IV, cap. 6.

Specimen foliiferum. Daphne Laureola Linn.

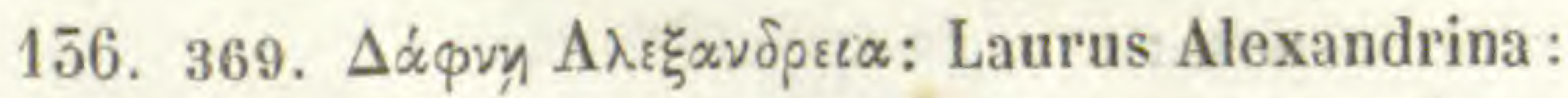

Lib. V, cap. 31: Rusco affinis tertia.

Specimen fructiferum. Streptopus amplexifolius Dec.

370. 'Ožuนupoivn: Oxymyrsine: Ruscus: Brusco: Pugnitopo:

Lib. V, cap. 28.

Specimen fertile. Ruscus aculeatus Linn. 


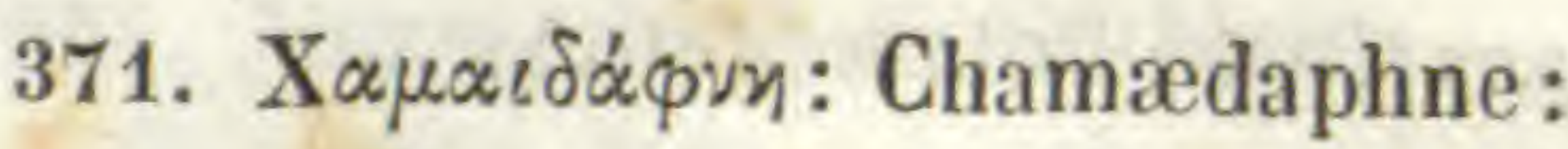

Lib. V, cap. 30: Rusco affinis altera. Specimen fructiferum. Ruscus Hypophyllum Linn.

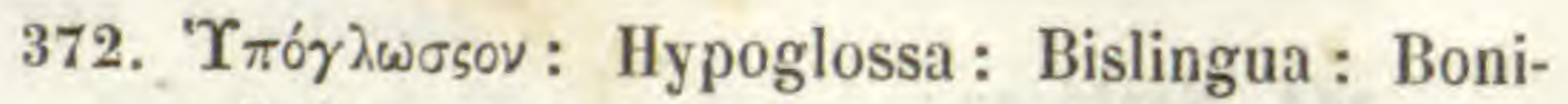
fatia :

Lib. V, cap. 29: Rusco affinis prima. Specimen fertile. Ruscus Hypoglossum Linn.

137. 373. Поһuyóvarov: Polygonaton; Frassinella:

Lib. V, cap. 32.

Specimen fertile. Polygonatum multiflorum All.

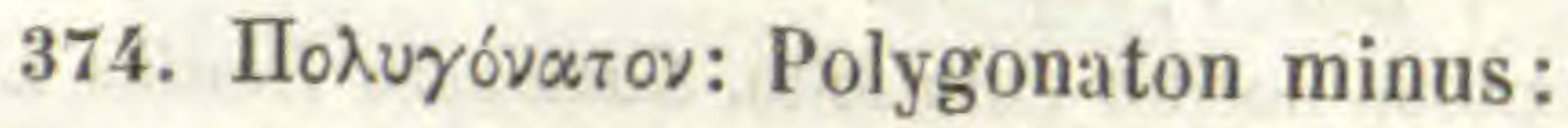

Lib. V, cap. 33: Polygon. angustifolium. Specimen sterile. Polygonatum verticillatum All.

138. 375. Lilium convallium: Mughetti:

Lib. V, cap. 34 .

Specimen sterile. Convallaria majalis Linn.

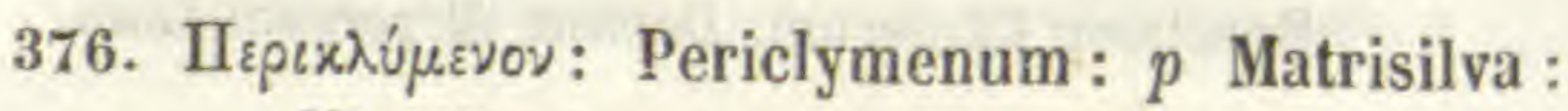

Vinciboseo:

Lib. V, cap. 11.

Specimen fructiferum. Lonicera Caprifolium Linn.

377. $\Sigma \mu i \lambda \alpha \xi \tau \rho \alpha \chi \varepsilon \tau \alpha$ : Smilax aspera: Edera spinosa:

Lib. V, cap. 12.

Specimen fructiferum. Smilax aspera Linn.

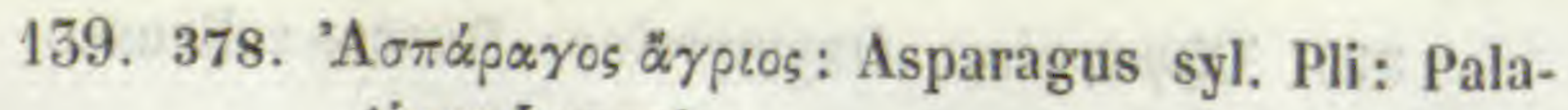
tium Leporis:

Lib. V, eap. 26.

Specimen sterile. Asparagus tenuifolius Lam. 
140. 379. "Axavtos: Acanthus: Branca orsina:

\section{Lib. VIII, cap. 24.}

Spica florifera, cum folio. Acanthus mollis Linn.

141. 380. $\Delta$ paxovtia $\mu \varepsilon \gamma \dot{\alpha} \lambda \eta$ : Dracunculus maior: Dragontea :

Lib. V, cap. 3 है.

Folium. Dracunculus vulgaris Schott.

381. "Apov: Aron: Gigaro:

Lib. V, cap. 36.

Spadix florifer, cum folio. Arum italicum Mill.

382. 'Apíapov: Arisarum:

Lib. V, cap. 37: Aron parvum.

Spadix fructifer, cum folio. Arisarum vulgare Targ.

142. 383. Aron alterum : Pro Colocasia:

Lib. V, cap. 38 .

Folium. Colocasia antiquorum Schott.

384. 'Apíapov: Arisarum alterum:

Lib. V, cap. 39: Arisaron primum.

Specimen floriferum. Biarum tenuifolium Schott.

383. Ophioglossa: Serpentina : Herba Lucciola:

Lib. XVI, cap. 17.

Specimen fertile. Ophioglossum vulgatum Linn.

143. 386. \} \Sigma _ { \tau } u _ { \chi } \chi ^ { \text { yos } } : \text { Strychnos: Solanum hortense: So- } latro:

Lib. V, cap. 19: Solatrum minus.

Specimen floriferum et fructiferum. Solanum nigrum Linn. 
387. Solanum arborescens;

Lib. V, cap. 23.

Specimen ut præcedens. Solanum Pseudocapsicum Linn.

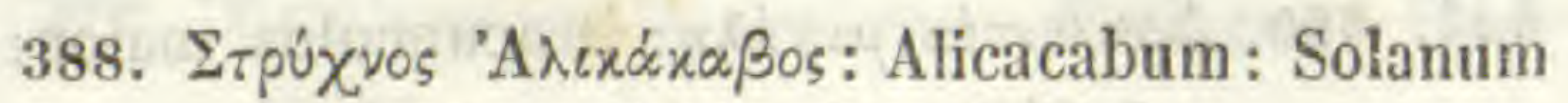
vesicarium: Alchechengi :

Lib. V, cap. 20.

Specimen fructiferum. Physalis Alkekengi Linn.

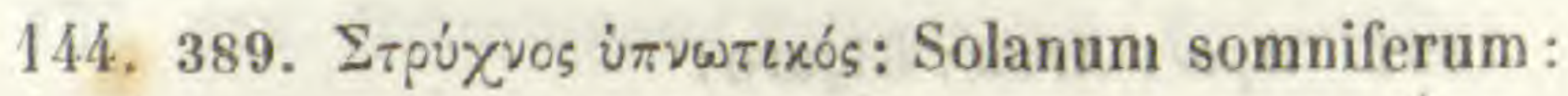

\section{Lib. V, cap. 21.}

Specimen floriferum et fructiferum. Physalis (Withania) somnifera Linn.

390. "A $\mu \pi \varepsilon \lambda$ os $\dot{\alpha} \gamma p i \alpha$ : Vitis sylvestris:

Lib. V, eap. 10: Salicastrum.

Specimen floriferum et fructiferum. Solanum Dulcamara Linn.

145. 391. Cucubalum. Pli: Solanum montanum: Solatro maggiore:

Lib. V, cap. 18.

Specimen floriferum. Atropa Belladonna Linn.

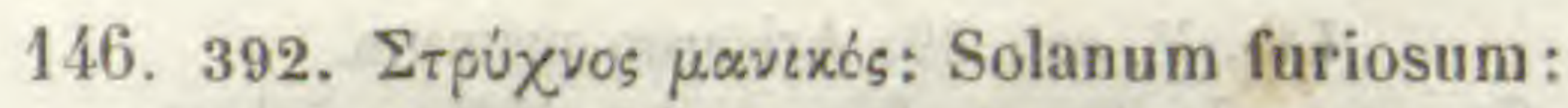

Lib. V, cap. 25: Barba Capri.

Specimen fructiferum. Actoea spicata Linn.

147. 393. Strychnon manicum. Pli: Stramonia: Mela spinosa :

$$
\text { Lib. V, cap. } 17 .
$$

Flos cum folio. Datura fastuosa Linn.

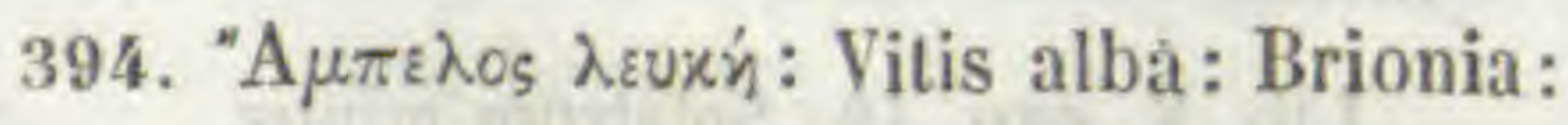

$$
\text { Lib. V, cap. } 7 .
$$

Specimen sterile. Bryonia dioica Jacq. 


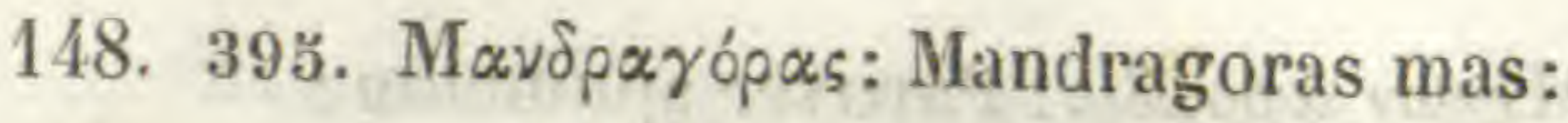

Lib. V, eap. 24: Mandragoras prima.

Specimen floriferum. Mandragora vernalis Bert.

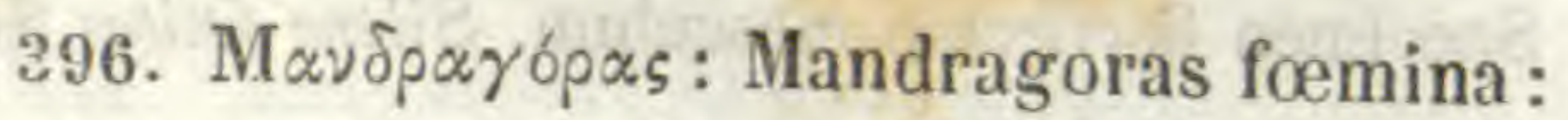

Lib. V, cap. 24: Mandragoras secunda,

Folium tantum, Mandragorce microcarpce Bert. referendum.

397. Móplov: Morion: Mandragoras $3^{a}$ :

Lib. V, cap. 24: Mandragoras tertia.

Flos cum folio. Mandragora officinarum Linn.

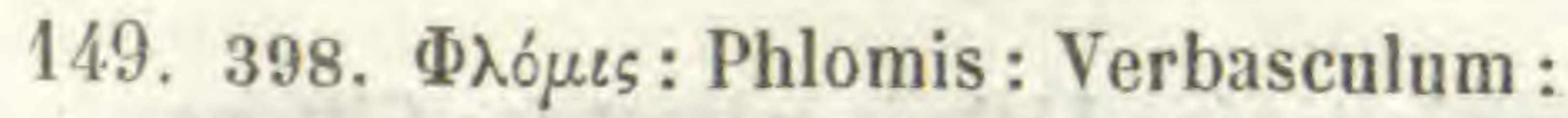

Lib. VI, cap. 57: Herba Paralysis altera.

Specimen fructiferum. Primula suaveolens Bert.

399. Ocimoides magnum:

Specimen floriferum, mas. Lychnis diurna? vel vespertina? Sibth.

400. ' $\Omega x \iota \mu \circ \varepsilon \delta_{c}^{\prime} s$ : Ocimoides : Ocimastrum:

Lib. VI, cap. 47: Ocymoides alterum.

Specimen floriferum. Saponaria Ocymoides Linn.

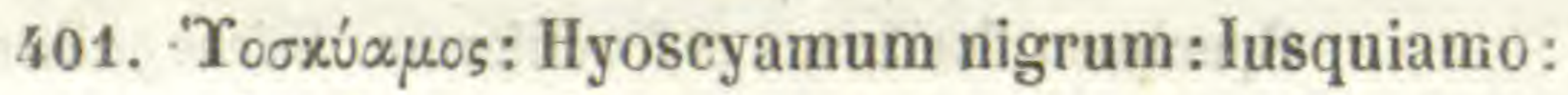

Dente cavallino:

Lib. VIII, cap. 45.

Specimen floriferum et fructiferum. Hyoscyamus niger Linn.

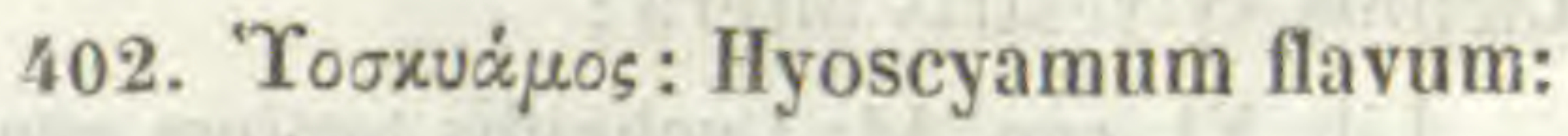

Lib, VIII, cap. 45: Hyoscyami alterum genus. Specimen ut præcedens. Hyoscyamus albus Linn.

150. 403. Saponaria :

Lib. VI, cap. 48.

Specimen floriferum. Saponaria officinalis Linn. 


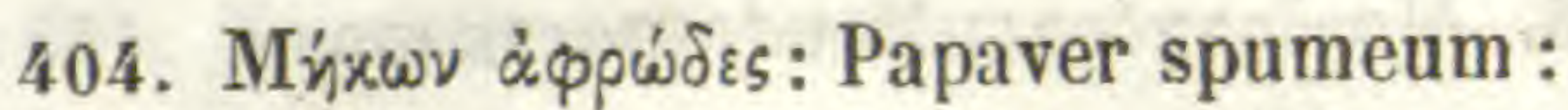

Lib. VI, cap. 49: Viscago (err. Niscago) secunda.

Specimen floriferum masculum. Silene Otites Smith.

405. Papaver spumeum:

Lib. VI, cap. 49: Viscago prima.

Specimen floriferum. Silene nutans Linn.

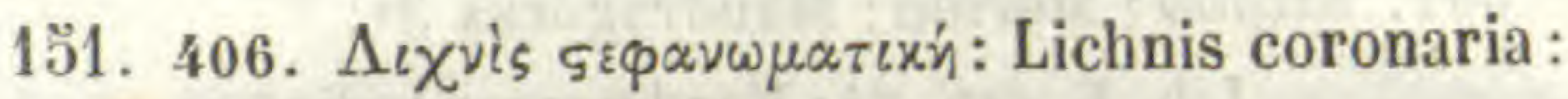

Lib. VI, cap. 43.

Specimen floriferum. Lychnis coronaria Lam.

407. $\Lambda_{\iota \chi} \chi^{\nu i s ~ \alpha ́ \gamma p i \alpha: ~ L i c h n i s ~ s y l v e s t r i s: ~ G i t t o n e: ~}$

Lib. VI, cap. 43.

Specimen floriferum. Lychnis Githago Lam.

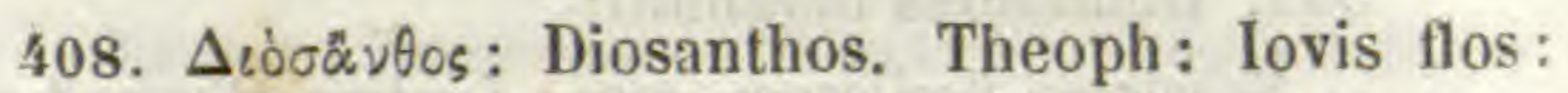
Fiorgharofano salvatico:

Lib. VI, cap. 45: Flos Gariotilus sylvestris.

Specimen floriferum. Dianthus Carthusianorum Linn.

152. 409. Odontides. Pli :

Lib. VI, cap. 52.

Specimen floriferum et fructiferum. Silene gallica Linn.

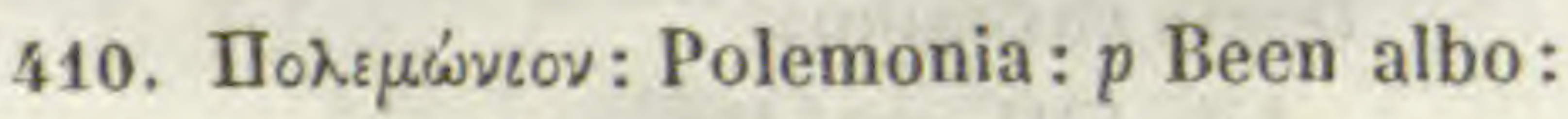

Lib. VI, cap. 50.

Specimen ut præcedens. Silene inflata Smith.

411. Lichnis sylvestris altera :

Lib. VI, cap. 44: Lychnidis tertium genus.

Specimen ut præcedentia. Silene Armeria Linn.

153. 412. Saxifraga Hermolai :

Lib. VI, cap. 46: Saxifraga prima.

Specimen floriferum. Asperulce cynanchica varietas quæ $A$, scabra Presl. 


\section{Saxifraga altera Hermol:}

Lib. VI, cap. 46: Saxifraga secunda. Specimen fructiferum. Sagina procumbens Linn.

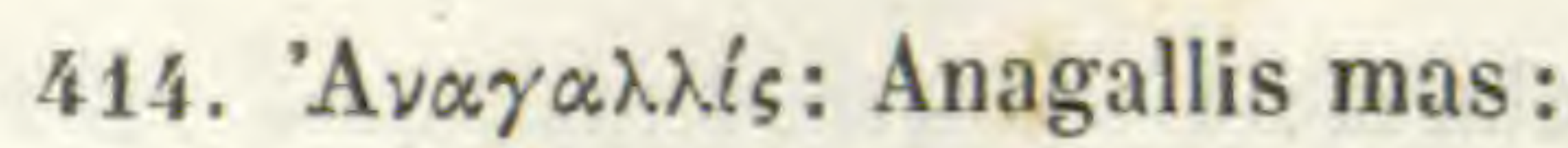

Lib. VI, cap. 59: A. flosculis colore puniceo.

Specimen floriferum et fructiferum. Anagalliszarvensis Linn.

154. 415. "А $\gamma \rho \omega \varsigma \tau \iota \varsigma ~ и ่ ~ \varepsilon \nu ~ \pi \alpha \rho v \alpha \sigma \varsigma \tilde{~: ~ G r a m e n ~ P a r n a s s i u m: ~}$

Lib. VI, cap. 53, p. 260 : Centunculus montanus. Specimen floriferum. Stellaria nemorum Linn.

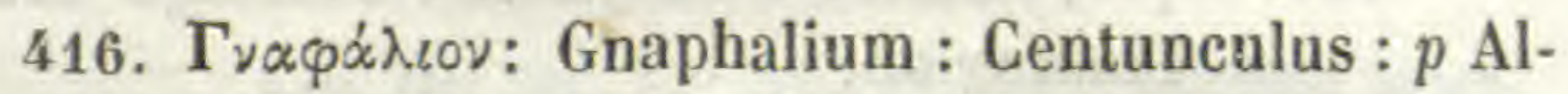
sine: Centonghio:

Lib. VI, cap. 53.

Specimen floriferum et fructiferum. Stellaria media Vill.

417. Gramen parnassium alterum: Lingua avis:

Lib. VI, cap. 53 , p. 260 : Centunculus montanus alter vel Lingua avis.

Specimen sterile. Stellaria Holostea Linn.

418. Gramen parnassium minus:

Lib. VI, cap. 53: Centunculi montani quartum genus?

Specimen floriferum. Cerastium arvense Linn.

419. Gnaphalion alterum:

Lib. VI, cap. 53, p. 260: Centunculi genus hirsutum, recto cauliculo?

Specimen floriferum et fructiferum. Cerastium glomeratum Thuill.

15๊. 420. Mupıó

Lib. VI, cap. 60: Myriophyllon primum.

Specimen floriferum. Hottonia palustris Linn. 
421. $\Lambda v \sigma \iota \dot{\alpha} \chi\llcorner$ เov: Lysimachia lutea:

Lib. VI, cap. 55: L. secunda racemosa.

Specimen floriferum. Lysimachia vulgaris Linn.

422. Nummularia:

Lib. VI, cap. 56.

Specimen sterile. Lysimachia Nummularia Linn.

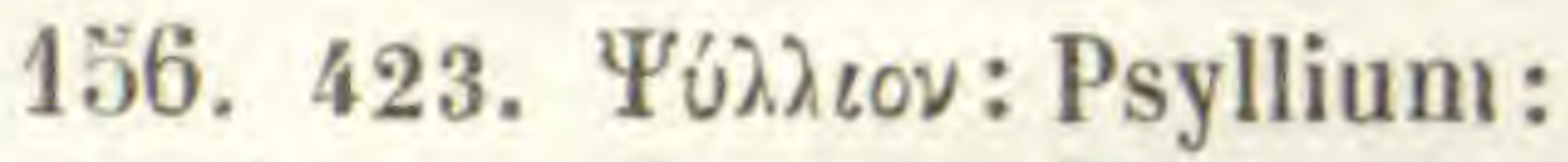

Lib. VIII, cap. 21: Psyllium maius.

Specimen floriferum. Plantago arenaria Waldst. Kit.

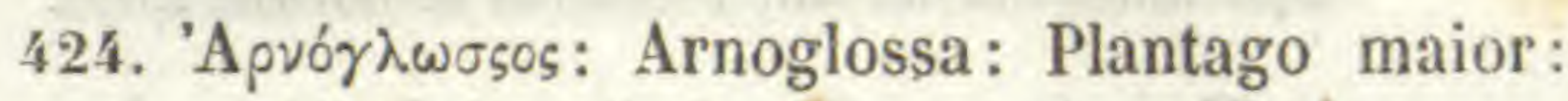

Piantagine:

Lib. VIII, cap. 16.

Specimen floriferum. Plantago major Linn., Decaisn. in Dec. Prodr.

425. Plantago media: Lanciola:

Lib. VIII, cap. 17.

Specimen fructiferum. Plantago lanceolata Linn.

157. 426. 'Oros\&ov: Holostium:

Lib. VIII, cap. 20: Coronopo affinis altera.

Specimen floriferum et fructiferum. Plantago Bellardi All.

427. 'A pvó $\gamma \lambda \omega \sigma s \circ s:$ Arnoglossa: Plantago minor:

Lib. VIII, cap. 16.

Specimen fructiferum. Plantago major, var. parva.

428. Kоршvо́лоus: Coronopus: Herba stella:

Lib. VIII, cap. 19.

Specimen floriferum. Plantago Coronopus Linn.

429. Orecchio di lupo:

Lib. VI, cap. 58: Auricula Ursi.

Folium tantum. Primula Auricula Linn. 
158. 430. Потирเоv: Poterium:

Lib. VI, cap. 40: Suffrutex Coroneolæ similis. Specimen floriferum. Genista germanica Linn.

431. Exoprios: Scorpius. Theoph : Nepa:

Lib. III, cap. 37: Spina Christi.

Specimen floriferum. Ulex europœeus Linn.

432. Corniola de Tintori :

Lib. VI, cap. 39: Coroneola.

Specimen floriferum. Genista tinctoria Linn.

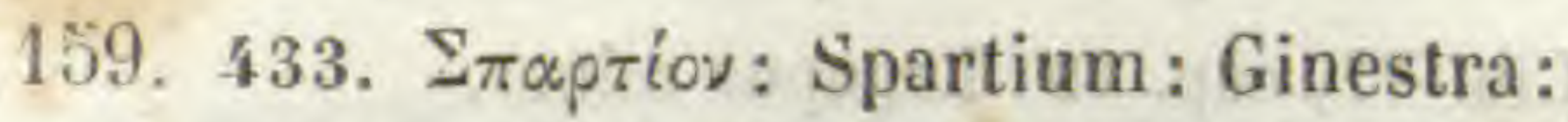

Lib. III, cap. 35 : Genista altera rotundo iunco. Specimen floriferum. Spartium junceum Linn.

434. Spartium parvum:

Lib. III, cap. 35: Genistæ quadrato iunco genus exiguum.

Specimen floriferum. Genista radiata Scop.

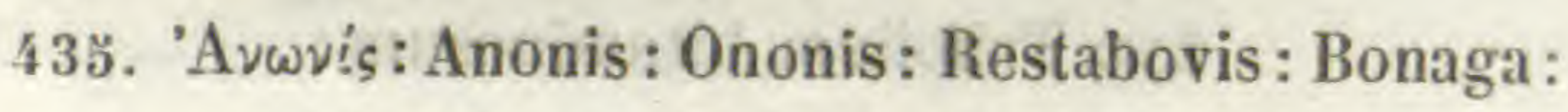

Lib. VI, cap. 21.

Specimen floriferum. Ononis spinosa $\beta$ Linn. Sp. ed. $2^{a}$;

O. campestris Koch. Ziz.

436. Tйìs: Telis: Fœnugræcum: Fien' græco:

Lib. VI, cap. 15.

Specimen fructiferum. Trigonella Fcenum grcecum Linn.

160. 437. Mทฺ่єxи́: Medica: Alfalfa:

Lib. VI, cap. 20.

Specimen floriferum. Medicago sativa Linn.

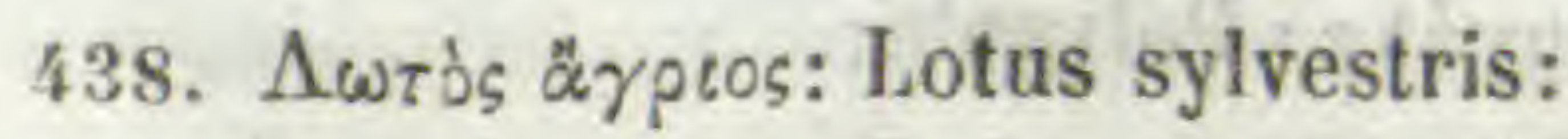

Lib. VI, cap. 17.

Specimen floriferum. Melilotus alba Desr. 
439. $\Lambda \omega \tau$ ช̀s ärpros: Lotus sylvestris: Tripuli:

Specimen floriferum, cum rudimentis fructuum. Iterum Melilotus alba.

161. 440. Medica altera:

Specimen floriferum et fructiferum. Medicago falcata Linn.

441. Lagopus montana:

Specimen fructiferum. Trifolium rubens Linn.

442. $\Lambda \alpha$ w'́nous: Lagopus: Leporaria herba:

Lib. VI, cap. 19: Lagopus minor.

Specimen floriferum et fructiferum. Trifolium arvense Linn.

443. Lagopus altera:

Specimen floriferum, juvene. Iterum Trifolium arvense.

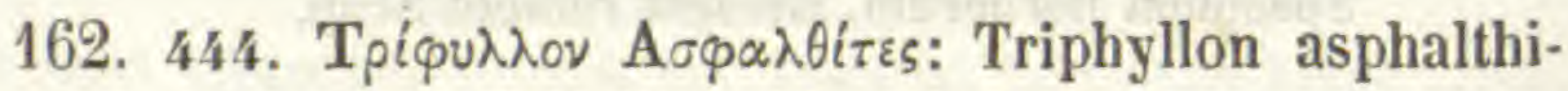
tes: Trifolium bituminosum :

Lib. VI, cap. 18.

Specimen floriferum. Psoralea bituminosa Linn.

163. 445. Гגаú̧: Glaux:

Lib. VI, cap. 23.

Specimen floriferum et fructiferum. Lotus (Bonjeania) hirsutus Linn.

446. Polygala altera: Melilotum Serap ${ }^{\text {nis }}$ :

Lib. VI, cap. 22.

Specimen floriferum. Lotus corniculatus Linn.

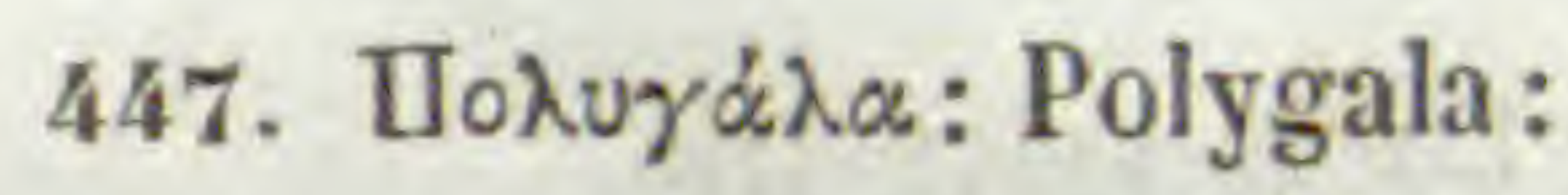

Lib. VI, cap. 25: Tragos, genus primum.

Specimen floriferum. Dorycnium herbaceum Vill. 
164. 448. Polygala altera:

Lib. VI, cap. 24.

Specimen floriferum. Tetragonolobus siliquosus Mœnch.

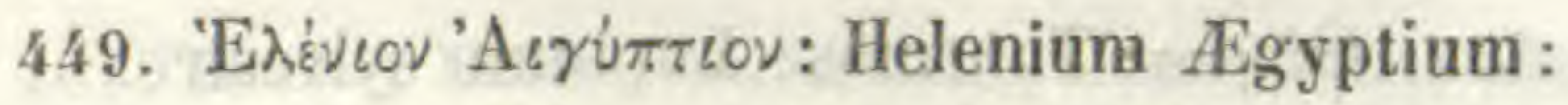

Lib. VI, cap. 27: Lupinus sylvestris primus. Specimen floriferum. Hedysarum coronarium Linn.

450. Orecchio di Lepre:

Lib. VI, cap. 29: Leporis auricula.

Specimen floriferum et fructiferum. Scorpiurus subvillosa Linn.

451. Herba humistrata : Humis: (1)

Lib. VI, cap. 38: Quædam in incultis nascens etc.

Specimen floriferum et fractiferum. Anthyllis tetraphylla Linn.

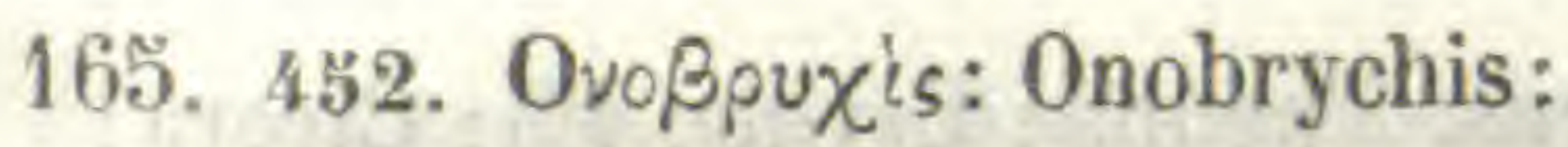

Lib. VI, cap. 35.

Specimen floriferum. Orobus niger Linn.

453. Onobrychis altera: Gralega: Lavanese:

Lib. VI, cap. 34 .

Specimen floriferum. Galega officinalis Linn.

454. Tpißonos: Tribulus terrestris. Theoph:

Lib. IV, cap. 27.

Specimen floriferum cum rudimento fructus. Tribulus terrestris Linn.

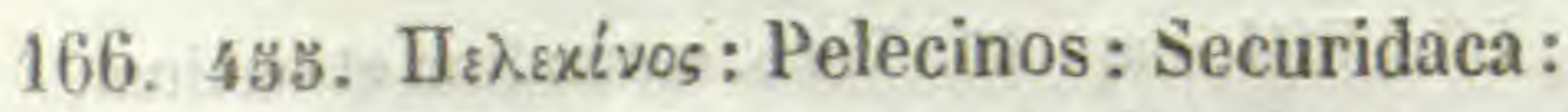

Lib. VI, cap. 30.

Specimen fructiferum. Bonaveria Securidaca Scop.

(I) Nomina aliena manu seripta. In indice desunt. 
456. Securidaca altera :

Lib. VI, cap. 31 .

Specimen floriferum. Coronilla varia Linn.

457. @épuos: Thermos: Lupinum sylvestre:

Lib. VI, cap. 26.

Specimen floriferum et fructiferum. Lupinus angustifolius Linn.

167. 458. 'A spáràos: Astragalus:

Specimen floriferum, ut duo sequentia. Vicia Pseudocracca Bert.

459. Astragalus:

Vicia dasycarpa Ten!

460. Astragalus:

Vicia tenuifolia Roth.

168. 461. ఉ’xpós: 0chrus. Theoph: Ervilia. Pli : Cicera : Rubiglo salvatico:

Lib. VI, cap. 6: Ervilii genus sylvestre. Specimen floriferum. Lathyrus latifolius Linn. Spec.

462. Aphaca montana:

Lib. VI, cap. 35: Onobrychis altera.

Specimen sterile. Orobus variegatus Ten.

169. 463. Фһб́́ Mas: Tasso barbasso dal fior bianco: Guaraguasco:

Lib. VIII, cap. 46: Verb. secundum et tertium. Specimen, ut in aliis hujus generis speciebus subsequentibus, e racemo florifero et folio radicali constans. Omnia vero specimina sunt imperfecta, corollis carentia. Verbascum thapsiforme Schrad. 


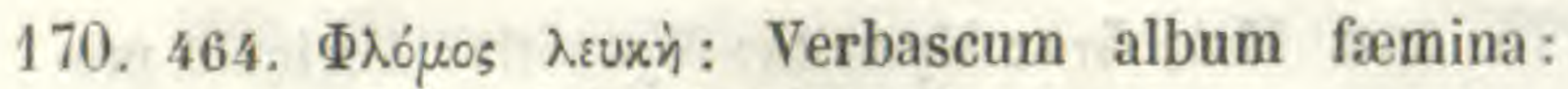
Plata domini : Piatandomino:

A præcedente videtur non differre.

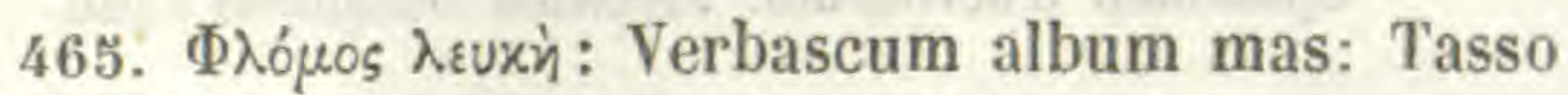
barbasso: Guaraguasco:

Lib. VIII, cap. 46: Verbascum sextum.

Verbaseum sinuatum Linn.

171. 466. $\Phi \lambda^{\prime} \mu_{\mu} \mu^{\prime} \mu_{\lambda} \alpha_{5}:$ Verbascum nigrum fæmina:

Tasso barbasso dal fior bianco:

Lib. VIII, cap. 46: Verbascum quintum?

Verbascum Lychnitis Linn.

467. $\Phi \lambda^{\prime} \mu_{0} \mu_{\varepsilon}^{\prime} \lambda_{\alpha \varsigma}$ : Verbascum nigrum mas: Blattaria. Pli :

Lib. VIII, cap. 46 : Verbascum nonum.

Verbascum Blattaria Linn.

468. $\Phi \lambda^{\prime} \mu_{0} \mu^{\prime} \hat{c}_{\lambda} \alpha_{s}:$ Verbascum nigrum fæmina: Blattaria altera :

Lib. VIII, cap. 46.

Verbascum virgatum With.

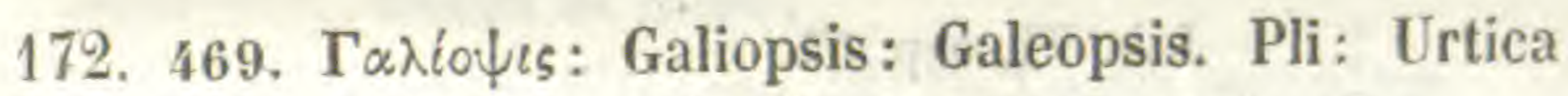
morta:

Lib. VIII, cap, 48.

Specimen floriferum et fructiferum. Scrophularia peregrina Linn.

470. Galeopsis altera: Scrofularia maggiore:

Lib. VIII, cap. 49: Scroful. maior altera.

Specimen ut præcedens. Scrophularia nodosa Linn.

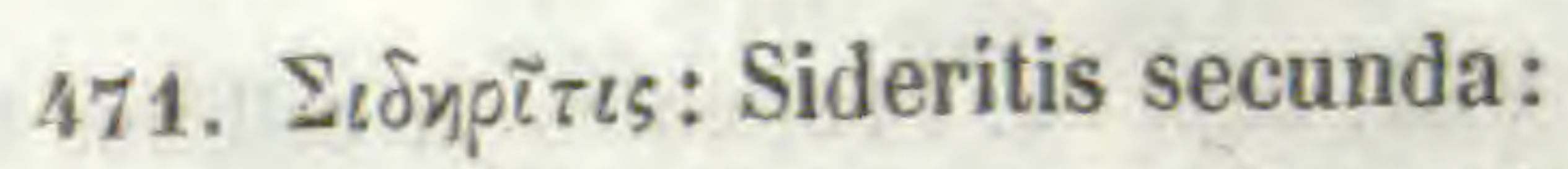

Lib. VIII, cap. 50: Scrofulariæ affinis minor.

Specimen fructiferum. Scrophularia canina Linn. 


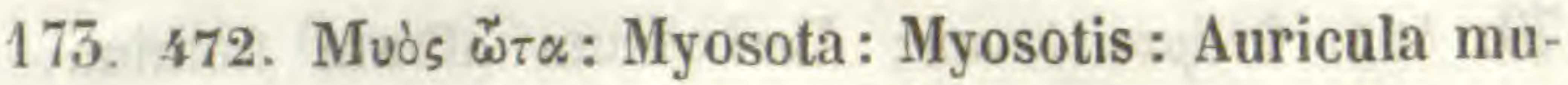
ris : Anagallis aquatica :

Lib. VIII, cap. 28.

Specimen fructiferum. Veronica Anagallis Linn.

473. Digitalis: Virga regia:

Lib. VIII, cap, 47: Virga regia prima.

Specimen floriferum. Digitalis lutea Linn.

174. 474. Anagallis sylvestris:

Lib. VIII, cap. 27: Auricula muris altera.

Specimen floriferum et fructiferum. Veronica arvensis Linn.

475. 'Avríṕp่เvov: Antirrhinon: Anagallis sylvestris:

Lib. VIII, cap. 26: Antirrhinon primum.

Specimen fructiferum. Veronica hederifolia Linn.

476. Anagallis sylvestris:

Lib, VIII, cap. 26: Auricula muris prima.

Specimen floriferum et fructiferum. Veronica serpyllifolia Linn.

477. Veronica fæmina:

Lib. VI, cap. 57.

Specimen floriferum. Linaria spuria Mill.

175. 478. "Osupes: Osyris:

Lib. VIII, cap. 14: Fruticulus in collibus linifolius, floribus purpureis.

Specimen floriferum, ut duo quæsequuntur. Polygala nicaensis Koch Deutsch.fl.V, p. 68 (excl. syn.et fig.cit.).

479. "O-upıs: Osyris:

Lib. VIII, cap. 14: Fruticulus in collibus linifolius, floribus pallescentibus.

Polygala flavescens Dec. 
480. "Oøupıs: Osyris:

Lib. VIII, cap. 14: Fruticulus in collibus linifolius, floribus cœruleis.

Polygala vulgaris Linn.

481.

Lib. VIII, cap. 51: Linaria secunda.

Specimen fructiferum. Linaria minor Desf.

482. Veronica mas:

Lib. VIII, cap. 27: Auricula muris tertia.

Spica cum floribus et fructibus. Veronica offcinalis Linn.

176. 483. Chamæence: $p$ Osyride: Linaria:

Lib. VIII, cap. 51.

Specimen floriferum. Linaria vulgaris Mill.

484. Chamæpeuce: Bocca di lione:

Lib. VIII, cap. 52: Os Leonis quartum in vineis.

Specimen floriferum et fructiferum. Antirrhinum Orontium Linn.

485. X $\alpha \mu \alpha t \pi \varepsilon \dot{x} x$ : Chamæpeuce : $p$ Antirrhino : Bocea di lione:

Lib. VIII, cap. 52: Os Leonis secundum.

Specimen ut præcedens. Antirrhinum latifolium Dec.

177. 486. Linaria montana:

Lib. VIII, cap. 52: Os Leonis tertium in montibus similius Linariæ?

Specimen floriferum. Linaria purpurea Mill.

487. Crista quædam in alpibus. (1)

Lib. VIII, cap. 38 ?

Specimen fructiferum. Pedicularis foliosa Linn.

(1) Nomen alienæ manus. 
178. 488. Eufragia maggiore:

Lib.VIII, cap. 37 : Cristee aliud genus, ramosum. Specimen floriferum et fructiferum. Rhinanthus minor Ehrh.

489. Eufragia :

Lib. VIII, cap. 35: Crista prima.

Specimen floriferum. Bartsia viscosa Linn.

490.

Lib.VIII,cap. 36: Cristæ aliud genus, secundum.

Frustulum sterile. Odontites lutea Reichenb.

491. Eufragia :

Lib. VIII, cap. 34 .

Specimen floriferum et fructiferum. Euphrasia officinalis Linn.

492. Amellum Vergilii :

Lib. VIII, cap. 35: Crista altera.

Specimen floriferum. Melampyrum arvense Linn.

179. 493. Saxifraga alba :

Lib. VIII, cap. 31: Thelygono affinis herba. Specimen fructiferum. Saxifraga rotundifolia Linn.

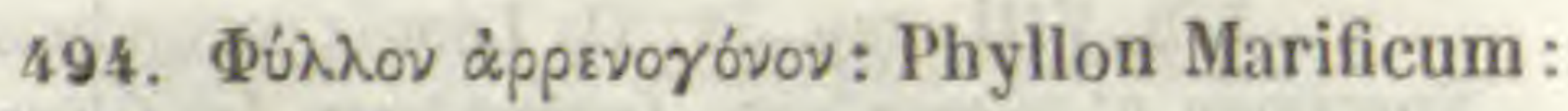

Lib. VIII, cap. 30: Phyllon primum.

Rosula foliorum radicalium. Saxifraga lingulata Bell.

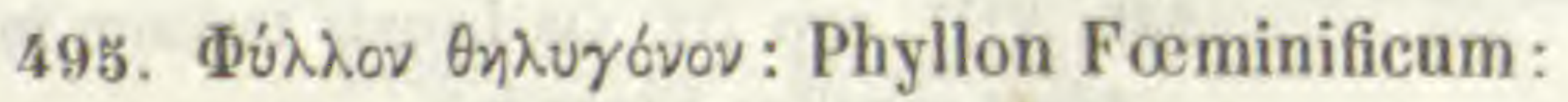

Lib. VIII, cap. 30: Phyllon primum.

Specimen floriferum et fructiferum. Saxifraga Aizoon Jacq.

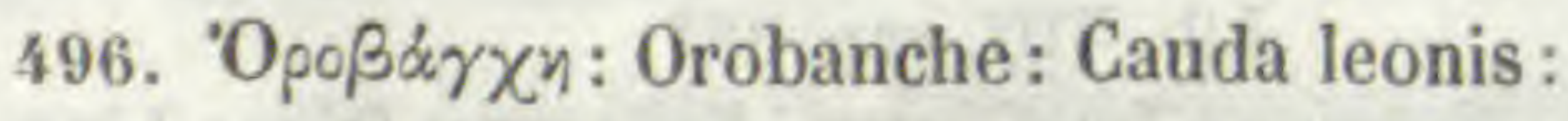

Lib. VIII, cap. 39: Orobanche prima.

Specimen floriferum. Orobanche minor Sutt. 


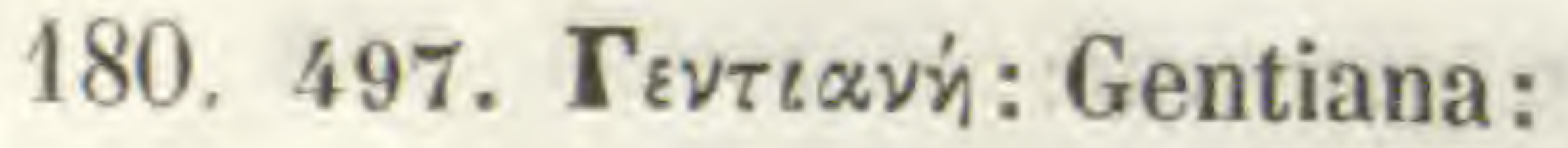

Lib. VI, cap. 78.

Folium radicale, Gentiance lutece Linn.

498. Gentiana:

Lib.VI, cap.79: Vincitoxicum Cruciata vocatum. Specimen floriferum. Gentiana Cruciata Linn.

499. Gentiana :

Lib. VI, cap. 79: Vincitoxicum tertium?

Specimen floriferum. Swertia perennis Linn.

500. Gentiana:

Specimen fructiferum. Valeriana saxatilis Linn.

501. Eufragia:

Lib. VIII, 34: Eufragia altera ramosa.

Specimen floriferum. Euphrasia officinalis Linn.

181. 502. Gentiana: Cruciata:

Lib. VI, cap. 79: Vincitoxicum primum,

Specimen floriferum. Gentiana asclepiadea Linn.

503. Gentiana:

Lib. VI, cap. 80: Gentianæ congener.

Specimen floriferum. Gentiana campestris Linn.

504. Splith:

Lib. VI, cap. 76.

Specimen floriferum, mancum. Corydalis ochroleuca Koch.

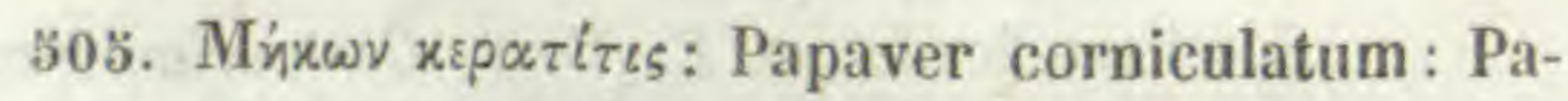
pavero cornuto:

Lib. VI, cap. 74.

Specimen floriferum et fructiferum. Glaucium luteum Scop. 
506. Leontopetalon parvum:

Lib. VI, cap. 7ö: Leontopetalon aliud.

Folium tantum. Probabiliter Corydalis cava Schweigg. et Koert.

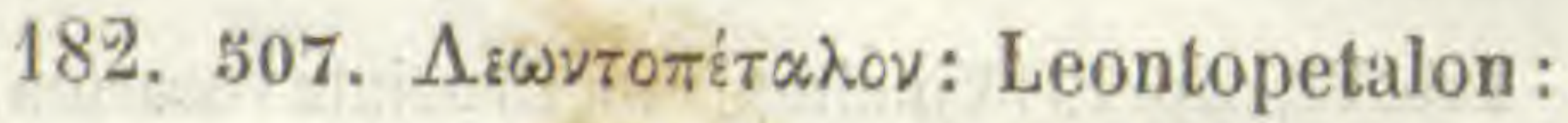

Lib. VI, cap. 75: Leontopetalon primum.

Folium. Leontice Leontopetalon Linn.

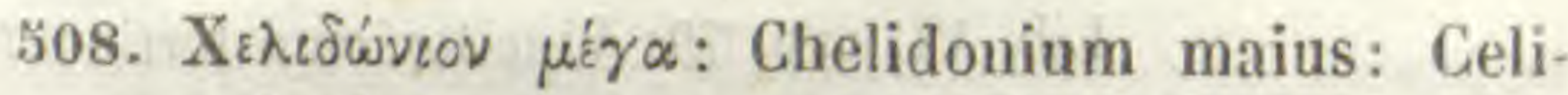
donia :

Lib. VI, cap. 73.

Specimen fructiferum. Chelidonium majus Linn.

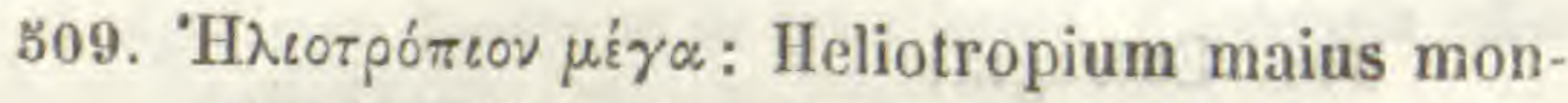
tanum : Scorpiurus:

Lib. VI, cap. 68: Catanance altera.

Specimen floriferum. Impatiens Noli tangere Linn.

183. 510. Eupatorium Mesues: Gratia dei :

Lib. VI, cap. 65.

Specimen floriferum. Gratiola officinalis Linn.

511. Achilleon. Pli: $p$ Centaurio maiori:

Lib. VI, cap. 64 .

Specimen floriferum. Chlora perfoliata Linn.

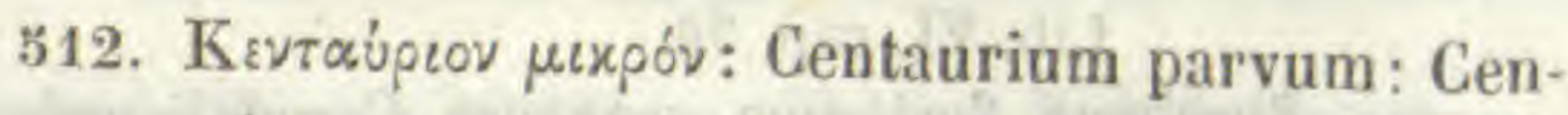
taurea :

Lib. VI, cap. 63.

Specimen floriferum. Erythrcea Centaurium Pers.

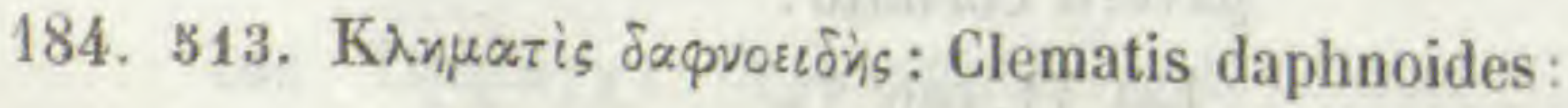

Lib. VIII, cap. 29: Provinca altera maior.

Specimen sterile. Vinca major Linn. 
514. Clematis daphnoides altera: Vincapervinca. Pli: Provinca :

Lib. VIII, cap. 29: Provinca prima. Specimen floriferum. Vinca minor Linn.

185. 515. 'A $\sigma x \lambda \eta \pi \iota \dot{\alpha} s:$ Asclepias: Vincetossico:

$$
\text { Lib. VI, cap. } 72 .
$$

Specimen fructiferum. Vincetoxicum officinale Mœnch, Decaisne in Dec. Prodr. VIII.

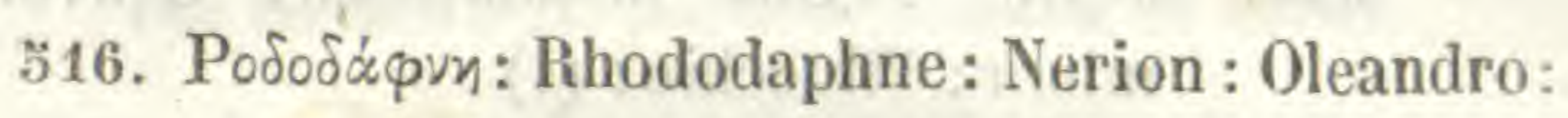

Lib. III, cap. 41.

Specimen floriferum. Nerium Oleander Linn.

186. 517. Periploca :

Lib. III, cap. 42.

Specimen floriferum. Periploca græca Linn.

518. 'A óxuvov: Apocynum: Periploca :

Lib. III, cap. 42 : Periploca altera.

Specimen ut præcedens. Marsdenia erecta $\mathbf{R}$. Br.

187. 519. 'O $\alpha \dot{\alpha} \gamma \rho \alpha$ : Onagra:

Lib. VI, cap. 70.

Specimen floriferum. Epilobium spicatum Lam.

ร20. Onagra minor:

Lib. VI, cap. 71: Onagra alia secunda.

Specimen floriferum. Epilobium tetragonum Linn.

188. \$21. Onagra palustris:

Lib. VI, cap. 71: Onagra alia tertia.

Specimen floriferum et fructiferum. Epilobium montanum Linn. 
522. Onagra palustris:

Lib. VI, cap. 71: Onagra alia prima.

Specimen floriferum. Epilobium hirsutum Linn. et Auct. recent.

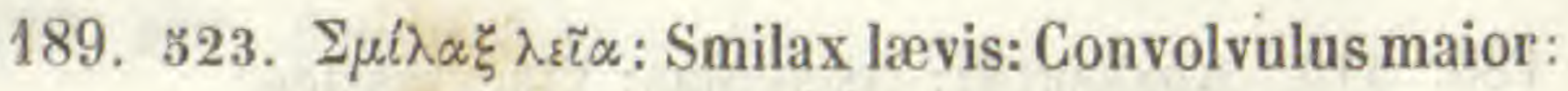

Lib. IX, cap. 25.

Specimen floriferum et fructiferum. Convolvulus ( $\mathrm{Ca}$ lystegia) sepium Linn.

524. Eגร̧iขn: Elxine Cissampelos: Convolvulus minor: Vilucchio: (1)

Lib. IX, cap. 24.

Specimen floriferum. Convolvulus arvensis Linn.

190. 525. Dorycnium parvum:

Lib. IX, cap. 27: Dorichnium alterum.

Specimen floriferum. Convolvulus Cantabrica Linn.

526. Dopúxveov : Dorycnium :

Lib. IX, cap. 27.

Specimen floriferum. Convolvulus Cneorum Linn.

527. K $\rho \alpha \mu \beta \eta \theta \alpha \lambda \alpha \sigma \varsigma i \alpha$ : Brassica marina: Soldanella:

Lib. IX, cap. 26.

Specimen floriferum et fructiferum. Convolvulus ( $\mathrm{Ca}$ lystegia) Soldanella Linn.

191. 528. Scamonium maius:

Lib. IX, cap. 23: Convolvuli alterum genus.

Specimen floriferum. Pharbitis Nil Chois.

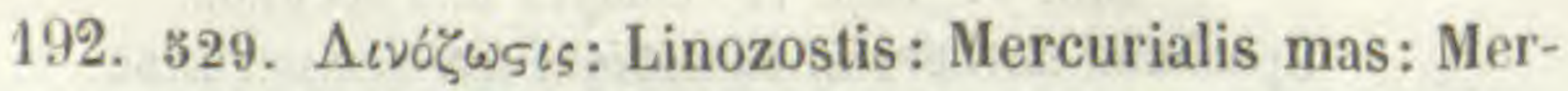
corella :

Lib. VIII, cap. 2.

Specimen floriferum et fructiferum. Mercurialis anпua fremina.

(1) Nomen postremum huic specimini et præcedenti interpositum. 


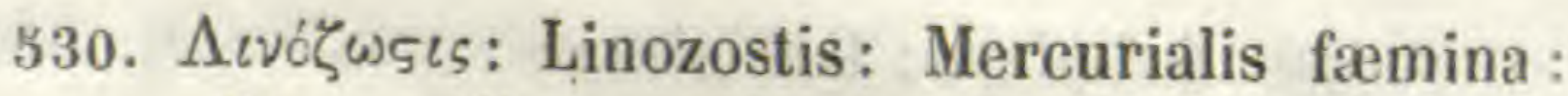
Mercorella:

Specimen floriferum. Mercurialis annua mas.

531. Mercurialis montana:

Lib. VIII, cap. 2.

Specimen sterile. Mercurialis perennis Linn.

193. 532. Herba S. Alberti: Alberga :

Lib. VIII, cap. 65.

Duo tantum folia. Barbarea vulgaris R. Brown.

533. $\Lambda \alpha \mu \Psi \dot{\alpha} \nu \eta$ : Lapsana: Radicetta salvatica:

Lib. VIII, cap. 56.

Specimen floriferum cum fructibus junioribus. Raphanus Raphanistrum Linn.

в34. Radicetta marina:

Lib. VIII, cap. 63: Erucæ genus.

Specimen floriferum. Cakile maritima Scop.

194. 535. Sinapis:

Lib. VIII, cap. 69: Sisymbrio affinis altera?

Specimen floriferum. Nasturtium anceps Dec.

536. 乏i $(\nu \eta \pi \iota:$ Sinapis: Senapa :

Lib. VIII, cap. 61 : Sinapi prima.

Caulis florifer cum folio radicali. Brassica nigra Koch.

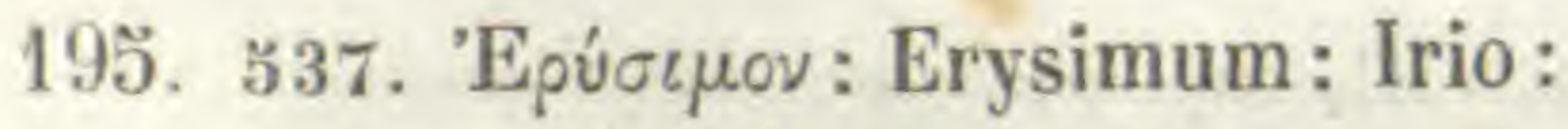

Lib. VIII, cap. 64 .

Specimen floriferum et fructiferum. Sisymbrium offcinale Scop.

538. Erysimum alterum:

Lib. VIII, cap. 64: Irio tertia.

Specimen ut præcedens. Sisymbrium polyceratium Linn. 
339. $\Delta \varepsilon \lambda$ фiveov: Delfinium:

Lib. VIII, cap. 67.

Specimen floriferum. Arabis hirsuta Scop. (sagittata Dec.).

196. 540. Thlaspi:

Lib. VIII, cap. 77: Thlaspi Creticum.

Specimen floriferum. Iberis umbellata Linn.

541. $\Theta \lambda \alpha \sigma \pi \iota:$ Thlaspi maius. Cratevæ: Raphano:

Folium radicale, cum frustulis duobus floriferis. $\mathrm{CO}_{0-}$ chlearia Armoracia Linn.

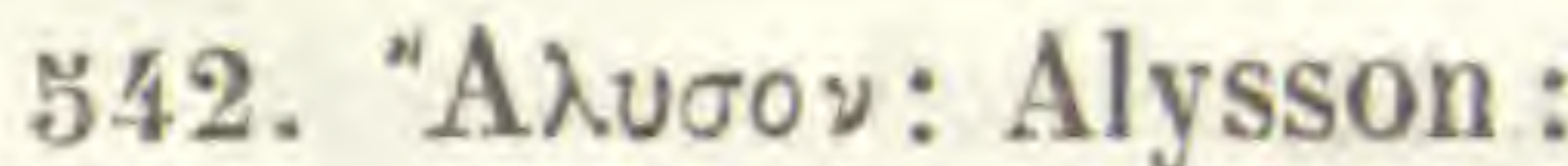

Plane deficiens. Anne Alyssum campestre?

197. 543. $\Delta \rho \alpha \beta \eta$ : Draba: Arabis:

Lib. VIII, cap. 73.

Specimen floriferum. Lepidium Draba Linn.

344.

Specimen fructiferum. Sisymbrium pinnatifidum Dec.

545. Bursa pastoris:

Lib. VIII, cap. 74.

Specimen fertile. Thlaspi Bursa pastoris Linn.

546. Carara :

Lib. VIII, cap. 84.

Specimen fructiferum. Senebiera Coronopus Poir.

547. Hiberis altera :

Specimen sterile. Iberis semperflorens Linn.

198. 548. $\Lambda \varepsilon \pi i \delta$ ¿ov: Lepidium: Herba pepe:

Lib. VIII, cap. 72.

Specimen floriferum. Lepidium latifolium Linn. 


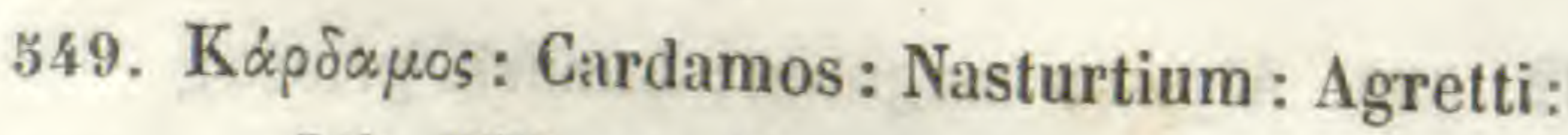
Lib. VIII, cap. 70.

Specimen floriferum et fructiferum. Lepidium sativum Linn.

550. 'I $\beta$ epis: Hiberis:

Lib. VIII, cap. 71.

Specimen ut præcedens. Lepidium graminifolium Linn.

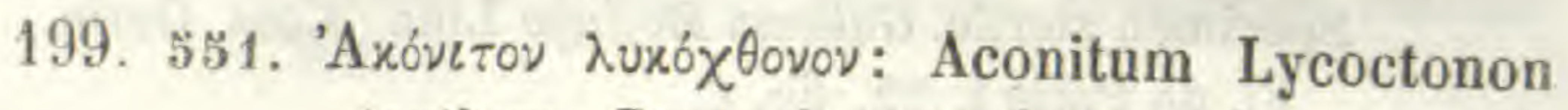
tertium: Dentaria maggiore:

Lib. XV, cap. 33.

Specimen fructiferum. Dentaria pinnata Lam.

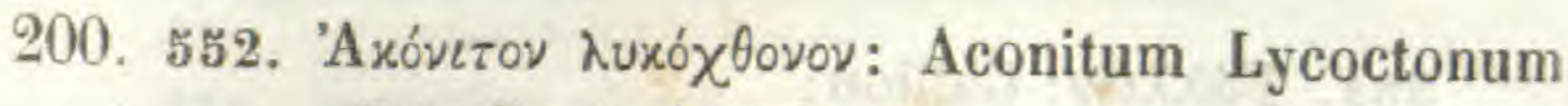
$3^{\text {aw }}$ : Dentaria minore:

Lib. XV , cap. 33.

Specimen floriferum. Dentaria bulbifera Linn.

553. Dentaria piccola:

Lib. XV, cap. 33: Dentaria minor altera.

Specimen floriferum. Cardamine chelidonia Linn.

554. $\Lambda$ Euxóiov äyptov: Leucoion sylvestre:

Lib. VIII, cap. 81 : Viola sylvestris lutea.

Specimen floriferum et fructiferum. Arabis alpina Linn.

201. 555. Alysson alterum : Lunaria minore:

Specimen fructiferum. Vesicaria utriculata Lam.

556. Alysson alterum: Lunaria minore:

Lib. VIII, cap. 82: Lunaria græca tertia.

Specimen fructiferum. Farsetia clypeata R. Brown.

557. Alliaria :

Lib. VIII, cap. 83.

Specimen fructiferum. Sisymbrium Alliaria Scop: 
202. 55s. Alysson magnum: Lunaria græca:

Lib. VIII, cap. 82 : Lunaria græca prima. Specimen floriferum, cum fructu ad latus. Lunaria rediviva Linn.

203. 559. 'Eputpóðavov: Erythrodanum: Rubia sylvestris: Robbia :

Lib. VIII, cap. 10 : Lappago.

Specimen sterile. Gallium Mollugo Linn; Coss. Germ. Fl. de Paris t. 22, fig. 6.

560. Rubia montana:

Specimen floriferum. Galium lovigatum Linn.

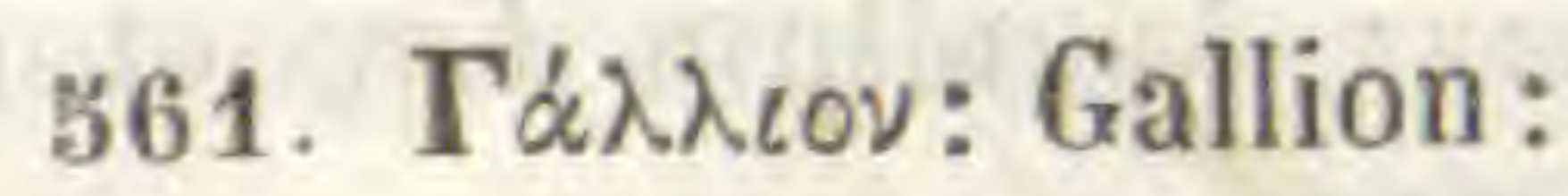

Lib. VIII, cap. 11.

Specimina duo commixta, alterum floriferum ad $G a-$ lium verum Linn., alterum vero fructiferum ad $G$. lucidum All. pertinens.

562. Crucialis:

Lib. VIII, cap. 12: Crucialis prima.

Specimen floriferum et fructiferum. Galium Cruciata Linn.

563. Crucialis marittima:

Lib. VIII, cap. 12: Crucialis tertia.

Specimen fructiferum. Vaillantia muralis Linn.

204. 564. Pyrola montana:

Lib. VIII, cap. 32.

Specimen floriferum. Pyrola minor Linn.

565. Pyrola palustris:

Lib. VIII, cap. 32.

Specimen floriferum. Samolus Valerandi Linn. 
566. Pyrola altera :

Lib. VIII, cap. 33 : Circea.

Specimen valde mancum, fructiferum. Circcea lutetiana Linn.

205. 568 (1). Halimum sylvestre. Hermolai:

Lib. IV, cap. 39: Herba juxta parietes nascens, semine Polygoni.

Specimen fructiferum mancum. Lepigonum rubrum Wahlb.

569. Tpáros: Tragos: Tragum: Scorpius. Pli:

Lib. IV, cap. 39: Salsola.

- Specimen floriferum. Salsola Kali Linn.

570. Scorpius altera:

Specimen floriferum. Speciei præcedentis varietas Iongifolia.

571. Halimum domesticum. Hermol:

Lib. IV, cap. 39: Isgarum.

Specimen sterile. Salsola Soda Linn.

572. Tragos altera:

Lib. IV, cap. 40 : Salsolæ aliud genus, primum. Specimen sterile. Salicornia herbacea Linn.

206. 373. ₹íxus ärplos: Cucumis sylvestris: Cocomero asinino:

Lib. V, cap. 6 .

Specimen fertile, sed valde mancum. Ecbalium. Elaterium Rich.

374. Balsamina: Caranza:

$$
\text { Lib. V, cap. } 4 .
$$

Specimen floriferum. Momordica Balsamina Linn.

(1) Numerus 567 deest, in numeratione oblitus, 


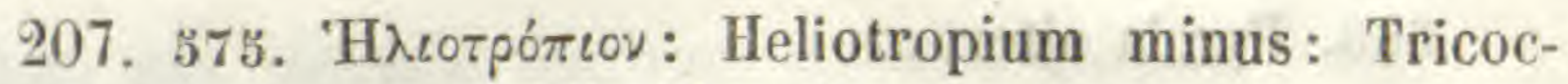
cum. Pli: Girasole piccolo:

Lib. IX, cap. 20.

Specimen valde mancum, foliis paucis tantum exstantibus. Crozophora tinctoria Adr. Juss.

576. $\Xi \alpha v \theta r o v:$ Xanthium: Lappa minore:

Lib. VIII, cap. 3.

Specimen floriferum et fructiferum. Xanthium strumarium Linn.

208. 577. Kixt: Cici : Ricinus: Girasole maggiore:

Lib. IX, cap. 19.

Specimen floriferum, mancum. Ricinus communis Linn.

209.

$$
\text { TiAvuaros: Tithymalus: }
$$

578. ò Kapaxias: Caracias: Esula maggiore:

Lib. IX, cap. 5: Characias primus.

Deest. Sequentia specimina huic generi pertinentia similiter maxima in parte aut desunt, imaginem tantum linquentia, aut adeo læsa sunt, ut eorum determinatio difficilis evadat. Euphorbia Characias Linn.

379. ò $\mu$ upritns: Myrtites parvus:

Euphorbia Pithyusa Linn.

580. ò Kuтapersias: Cyparissias: Esula minore:

Lib. IX, cap. 7: Esula prima.

Euphorbia Cyparissias Linn.

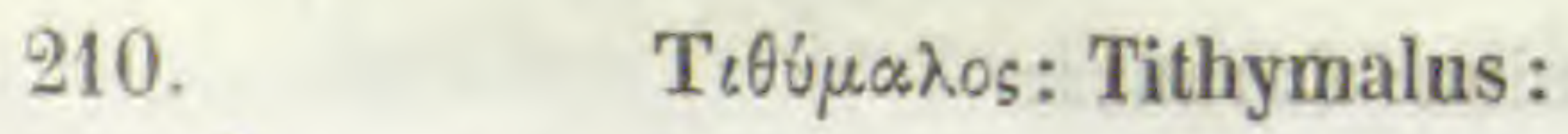

581. ó $\Delta \varepsilon \nu \delta \rho \omega \delta \eta \varsigma$ : Dendrodes: Arborescens:

Lib. IX, cap. 11.

Euphorbia dendroides Linn. 


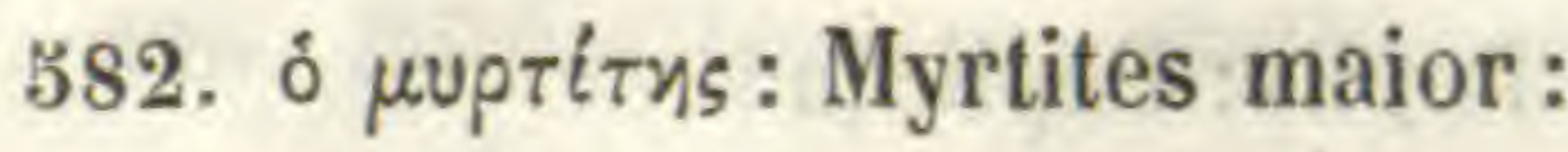

Lib. IX, cap. 10: Myrsinites altera.

Euphorbia nicceensis All.

583. ó $\pi \alpha \rho \alpha ́ \lambda \_$s: Paralius : Marittimus:

Lib. IX, cap. 9.

Euphorbia Paralios Linn. Amœen. acad. III, p. 129.

584. $\pi \dot{\varepsilon} \pi \lambda$ os: Peplus parva:

Lib. IX, cap. 13: Cataputia pusilla.

Euphorbia exigua Linn.

\section{Tetú $\mu \alpha \lambda$ os: Tithymalus :}

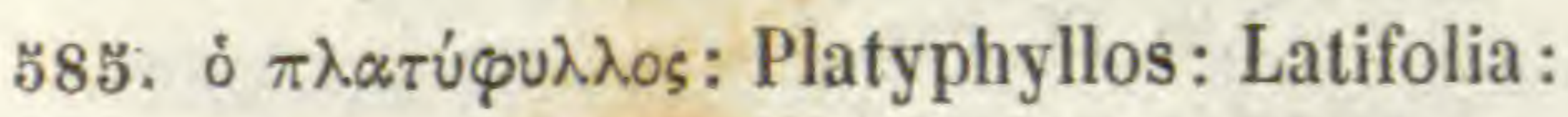

Lib. IX, cap. 12: Latifolium aliud.

Euphorbia pubescens Desf., Guss. Prodr. sic. II, p. 5๊51.

586. Esula rotunda:

Lib. IX, cap. 14: Helioscopius alter.

Euphorbia dulcis Linn., Jacq. Fl. austr.

212. 587. Caracias montana:

Lib. IX, cap. 5.

Euphorbia amygdaloides Linn.

588. MaAupis: Lathyris: Cataputia:

Lib. IX, cap. 13.

Euphorbia Lathyris Linn.

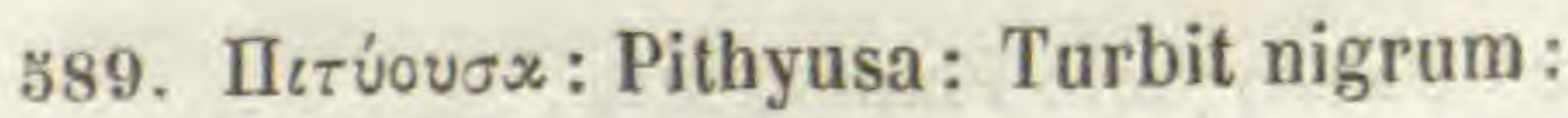

Lib. IX, cap. 6.

Euphorbia palustris Linn.

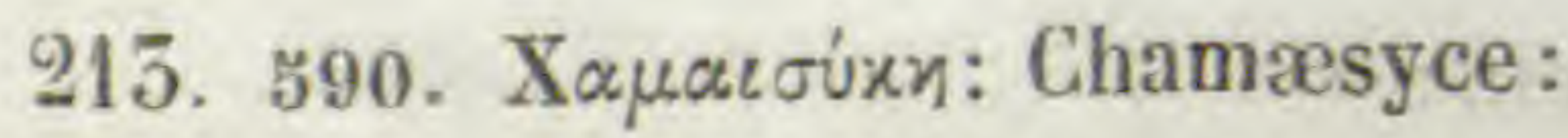

$$
\text { Lib. IX, cap. } 17 .
$$

Euphorbia Chamasyce var. canescens. 
591. II $\varepsilon \pi \lambda i_{s}$ : Peplis: Peplium:

Lib. IX, cap. 16.

Euphorbia Peplis Linn.

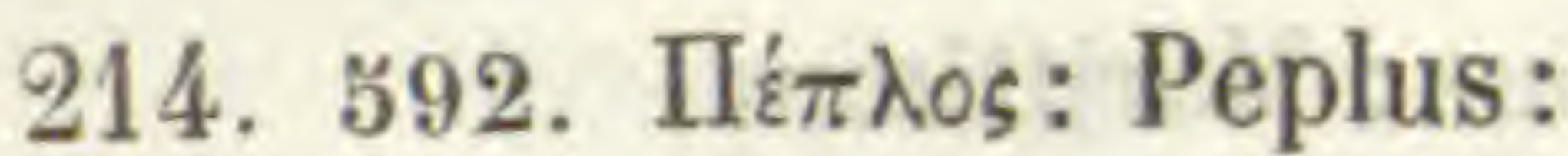

Euphorbia falcata Linn.

593. T⿺辶ú $\mu \alpha \lambda$ os ó $\dot{\eta} \lambda \iota 0 \sigma \times o ́ \pi \iota 0 s:$ Tithymalus Helioscopius :

Lib. IX, cap. 15: Peplus.

Euphorbia Peplus Linn.

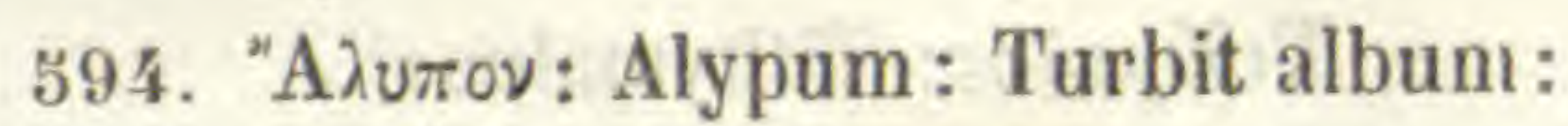

Lib. IX, cap. 8.

Euphorbia spinosa Linn.

215. 595. Asphodelus:

Lib. X, cap. 30 : Liliago maior.

Scapus florifer, cum folio radicali. Hemerocallis fulva Linn.

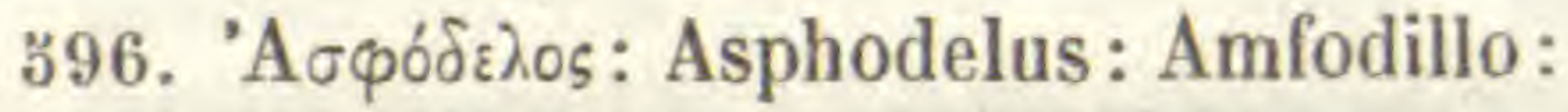

Lib. X, cap. 26: Asphodelus caule simplici.

Scapus florifer, cum folio radicali. Asphodelus albus Willd; Parl! Fl. ital. II, p. 604.

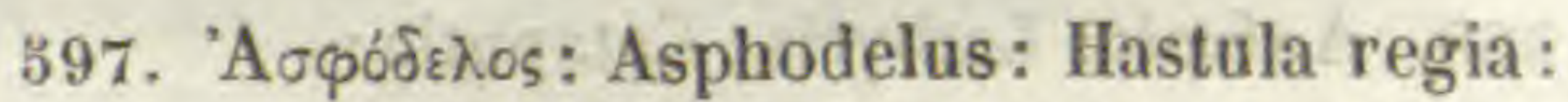

Lib. X, cap. 29: Asphodelus luteus.

Specimen tantum foliferum. Asphodeline lutea Reich.

216. 598. $\Phi \alpha \lambda \alpha \gamma \gamma$ เov: Phalangium:

Lib. X, cap. 27: Pbalang. cauliculis ramosis. Specimen floriferum. Phalangium ramosum Lam.

599. Phalangium:

Lib.X, cap. 27: Phalang. cauliculis simplicibus. Specimen floriferum. Phalangium Liliago Schreb. 


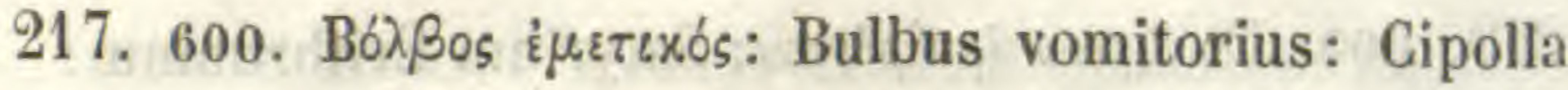
di serpe:

Lib. X, cap. 5: Cepa canina aut Anguina, prima.

Specimen floriferum. Leopoldia comosa Parl; Muscari comosum Mill.

601. Bulbine. Pli :

Lib. $\mathbf{X}$, cap. 4: Bulbus sylvestris.

Specimen floriferum. Botryanthus odorus Kunth.

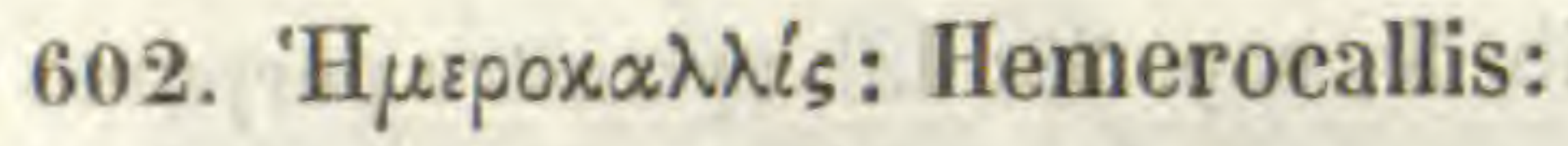

Lib. X, cap. 23: Narcissus marinus.

Flos cum foliis duobus. Pancratium maritimum Linn.

603. Hemerocallis altera:

Lib. X, cap. 21: Lonchitis.

Specimen floriferum. Tulipa sylvestris Linn.

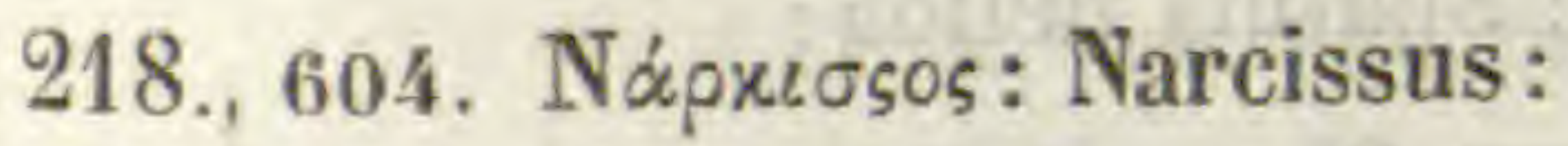

Lib. X, cap. 22: Narcissus alter.

Specimen floriferum. Narcissus aliquis e sectione $N$. Tazetice.

605. $\Upsilon \dot{\alpha} \alpha \iota \nu \theta_{05}$ : Hyacinthus:

Lib. X, cap. 7: Hyacinthi alterum genus.

Specimen floriferum. Scilla bifolia Linn.

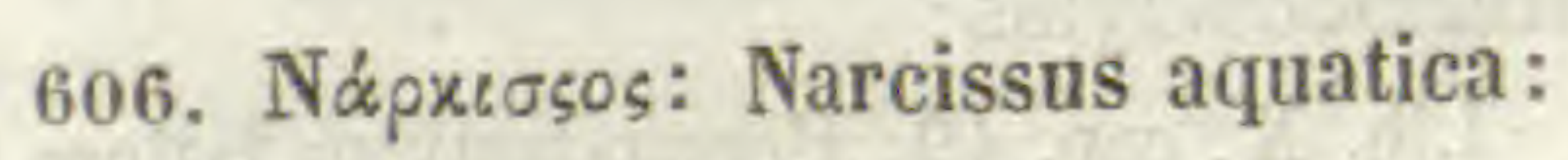

Lib. X, eap. 24: Narcissus aquaticus primus.

Specimen floriferum. Leucojum aestivum Linn.

607. Nגaptasos: Narcissus:

Lib. X, cap. 22: Narcissus primus.

Specimen floriferum. Narcissus poeticus Linn. 


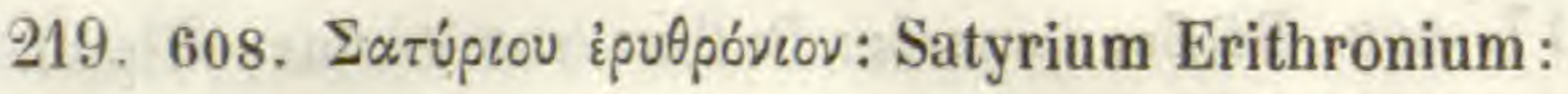

Lib. X, cap. 45.

Specimen floriferum, mancum. Hermodactylus tuberosus Salisb.

609. Kpivov äprov: Lilium sylvestre: Martagon:

Lib. X, cap. 33: Lilium sylvestre croceo flore. Specimen ut præcedens. Lilium bulbiferum Linn; $\boldsymbol{L}$. croceum Chaix.

610. Kpivov à rpıov: Lilium sylvestre: Martagon:

Lib. X, cap. 33: Lil. sylvestre purpureo flore. Specimen ut præcedens. Lilium Martagon Linn.

220. 611. Eıpis: Xiris: $p$ Acoro:

Lib. X, cap. 39: Acorus.

Specimen floriferum mancum, ut etiam sequens. Iris Pseud'Acorus Linn.

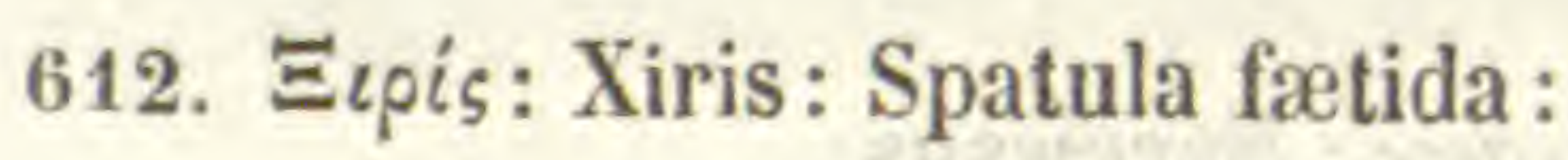

Lib. X, cap, 38.

Iris fatidissima Linn.

613. 'Ipes: Iris parva: Iris illirica:

Lib. $\mathbf{X}$, cap. 37.

Specimen floriferum. Iris graminea Linn.

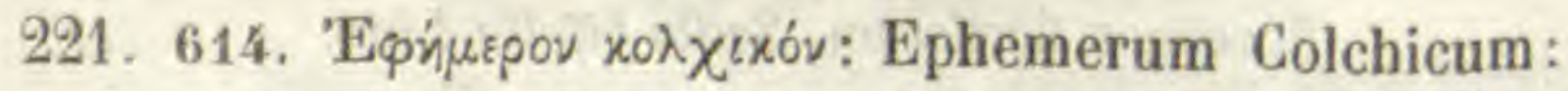
Hermodactylus:

Lib. X, cap. 19: Hermodactylus latiore folio. Specimen fructiferum, fructu immaturo. Colchicum autumnale Linn.

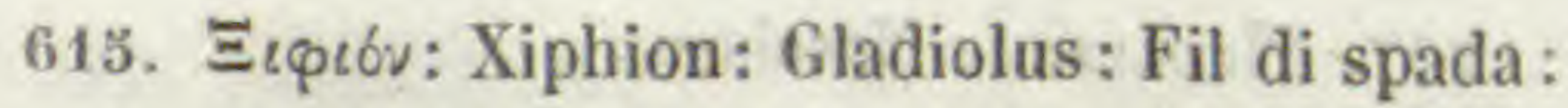

Lib. V, cap. 35 .

Specimen floriferum. Gladiolus segetum Gawl. 
616. Kpóxos: Crocus: Crocum sylvestre: Zappherano salvatico:

Lib. X, cap. 20.

Specimen fructiferum. Crocus vernus All.

222, 617. Gladiolus altera: Canna d' india:

Lib. X, cap. 34.

Folium. Canna indica Linn.

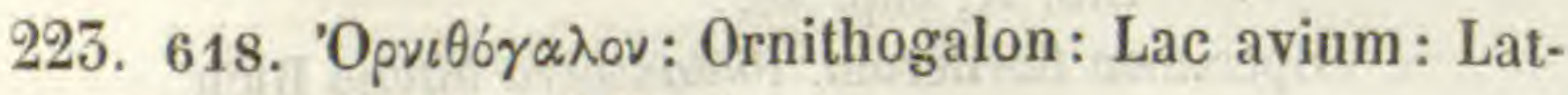
te di gallina:

Lib. X, cap. 10.

Specimen floriferum. Ornithogalum umbellatum Linn.

619. Ornithogalon:

Lib. X, cap. 11: Bulbus Ornithogalo similis, primus.

Specimen floriferum. Ornithogalum narbonense Linn.

620. Ornithogalon alterum:

Lib. X, cap. 11: Bulbus Ornithogalo similis, secundus.

Specimen floriferum. Ornithogalum pyrenaicum Linn.

224. 621. 'O фroбxópodov: Ophioscorodon: Allium anguinum: Aglo di serpe: (1)

Specimen floriferum, valde mancum. Allium roseum Linn.

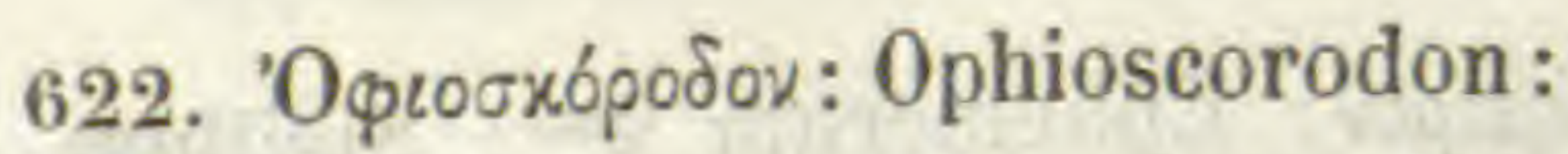

Umbella florifera. Allium pallens Linn.

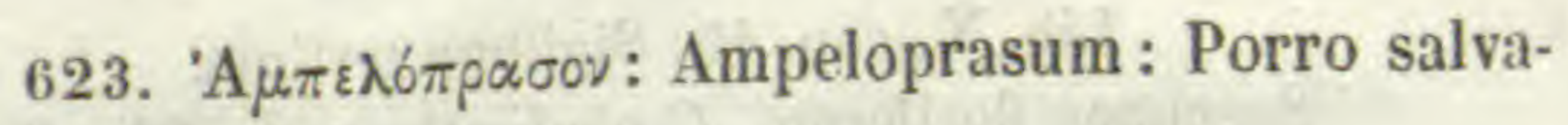
tico:

$$
\text { Lib. X, cap. } 13 .
$$

Specimen floriferum. Allium triquetrum Linn.

(1) Hæc duo ultima nomina posita inter præsens et sequens specimen. 


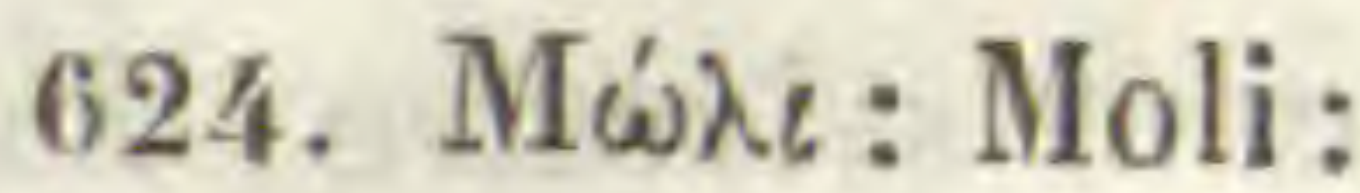

Lib. X, cap. 9.

Umbella florifera, cum folio, Allium magicum Linn. Spec. ed. $1^{\text {a }}$ et $2^{\mathrm{a}} ; A$, nigrum plur. auct.

225. Kuvòs öpxıs: Cynos orchis: Testiculus canis:

623. Satyrio:

$$
\text { Lib. X, cap. } 46 .
$$

Specimen floriferum. Platanthera bifolia Rich.

626. Satyrio:

Lib. X, cap. 46.

Specimen floriferum. Serapias Lingua Linn.

627. Satyrio:

Lib. X, cap. 46.

Specimen floriferum. Orchis laxiflora Lam.

628. Palma christi :

Lib. X, cap. 47.

Specimen floriferum. Orchis maculata Linn.

226. 629. $\mathbf{E} \varphi \dot{\mu} \mu \varepsilon$ роv: Ephemerum 2 ${ }^{\text {um }}$ : Sigillum solomonis :

Lib. $X$, cap. 49 : Sigillum primum.

Specimen sterile. Cephalanthera pallens Rich.

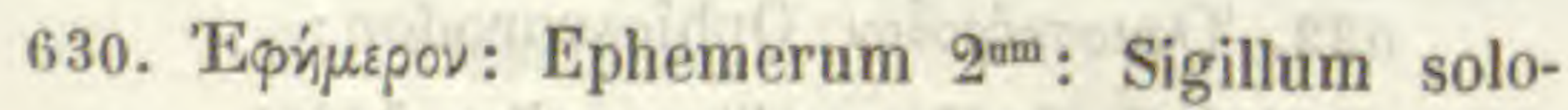
monis:

Lib. X, cap. 49: Sigillum tertium.

Specimen floriferum. Cephalanthera ensifolia Rich.

631. Ophris. Pli :

$$
\text { Lib. X, cap. } 48 .
$$

Specimen floriferum. Listera ovata R. Brown. 


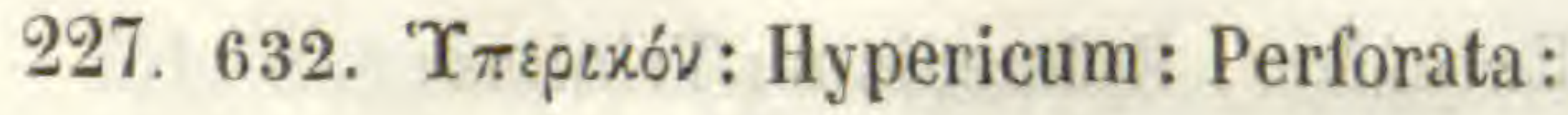

Lib. IX, cap. 41.

Specimen fructiferum. Hypericum perforatum Linn.

633. Androsæmum:

Lib. IX, cap. 42: Ascyroides secunda.

Specimen'fertile, sed valde mancum. Hypericum hirsutum Linn.

634. 'A бxupov: Ascyron:

Lib. IX, cap. 42.

Specimen floriferum. Hypericum tetrapterum Fries.

228. 635. Androsæmum:

Lib. IX, cap. 42: Ascyroides prima.

Specimen floriferum. Hypericum montanum Linn.

636. Androsæmum :

Specimen fructiferum. Hypericum Richeri Vill.

637. Kópıs: Coris:

Lib. IX, cap. 44.

Specimen floriferum et fructiferum. Hypericum Coris Linn.

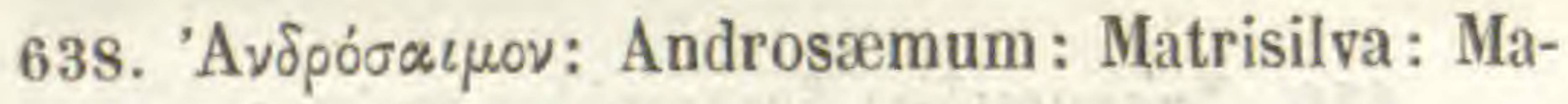
dreselva :

\section{Lib. IX, cap. 43.}

Specimen sterile. Androscemum officinale All.

229. 639 .

Lib. XV, cap. 17, p. 576: Genus quoddam alpinum etc.

Specimen floriferum. Parnassia palustris Linn.

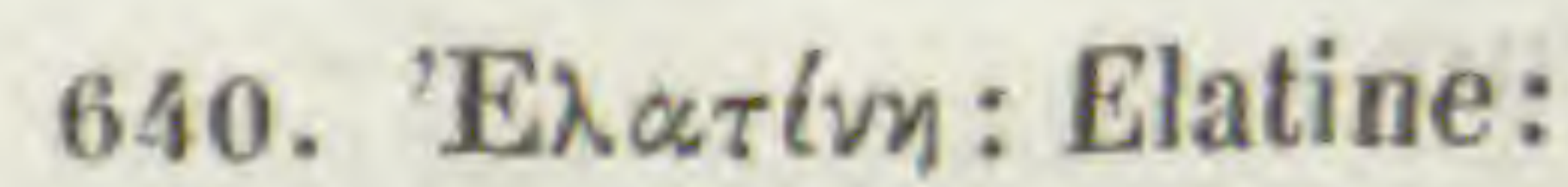

Lib. IX, cap. 32.

Specimen fructiferum. Specularia Speculum Dec. fil. 


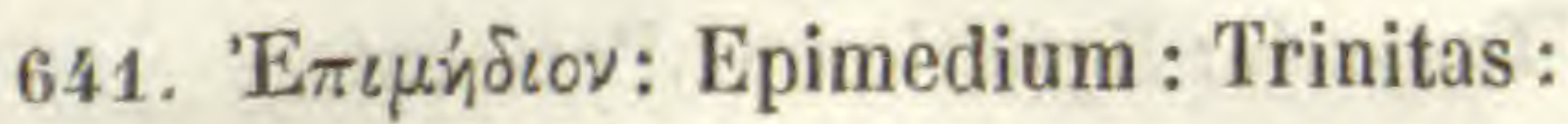

\section{Lib. XIV, cap. 8.}

Specimen fertile. Anemone Hepatica Linn.

642. "Aøapov: Asarum: Bacchara:

Lib. IX, cap. 36.

Specimen sterile. Asarum europceum Linn.

643. Faba inversa: Vesicaria dal cuore:

Lib. IX, cap. 21.

Specimen floriferum et fructiferum. Cardiospermum Halicacabum Linn.

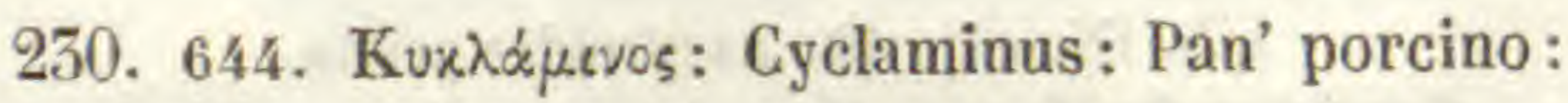

Lib. IX, cap. 38 : Cyclamini alterum genus . Specimen flore et foliis constans. Cyclamen repandum Sibth. Smith; C. vernum Gay.

645. $\Phi \lambda$ órıov: Phlogion. Theoph: Viola flammea : Iacea :

Lib. IX, cap. 40.

Specimen floriferum. Viola tricolor sylvestris.

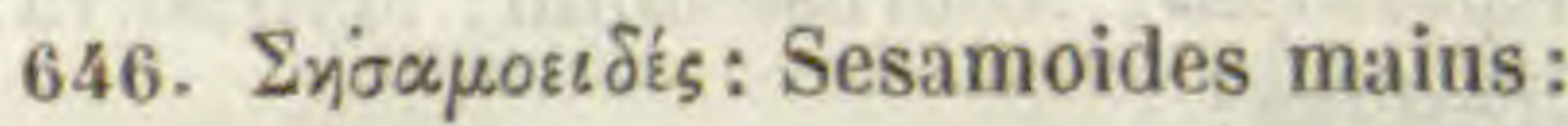

Lib. IX, cap. 34: Sesamoides alterum.

Specimen floriferum. Reseda lutea Linn.

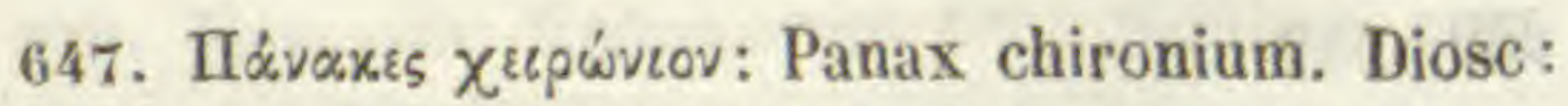

Lib. IX, cap. 46: Chironia prima.

Specimen floriferum. Helianthemum vulgare Gærtn.

648. Chironium alterum:

Lib. IX, cap. 45: Frutex simillimus Cori. Specimen fructiferum. Helianthemum Fumana Mill. 


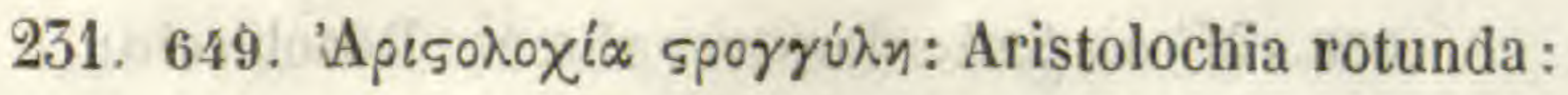

Lib. XV, cap. 5: Aristolochia prima.

Specimen floriferum. Aristolochia rotunda Linn.

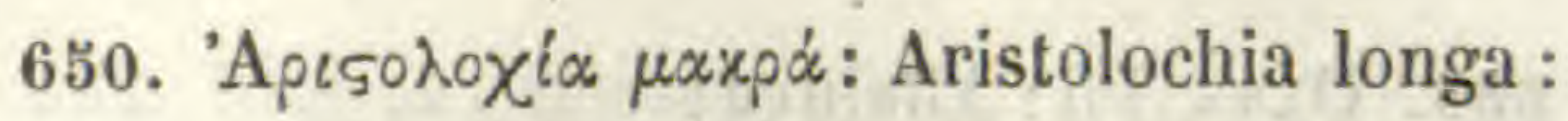

Lib. XV, cap. 5: Aristolochia altera.

Specimen ut præcedens. Aristolochia Clematitis Linn.

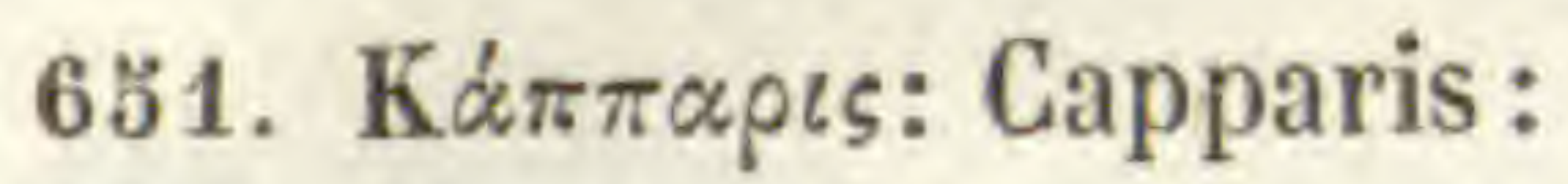

Lib. XV, cap. 6.

Omnino deest. Verisimiliter Capparis rupestris Sibth. et Smith, apud nos vulgaris.

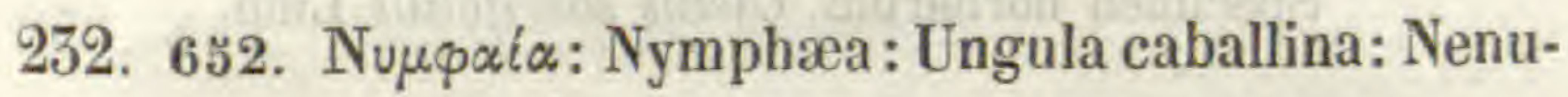
faro:

Lib. XV, cap. 7.

Flos et folium Nymphcece flore candido Cæs. I. c. quæ N. alba Linn,, cum fructu juniori Nymphcece flore luteo Cæs. quæ Nuphar luteum Smith.

6ə3. Nymphæa parva:

$$
\text { Lib. XV, cap. } 7 .
$$

Specimen floriferum. Hydrocharis Morsus rance Linn.

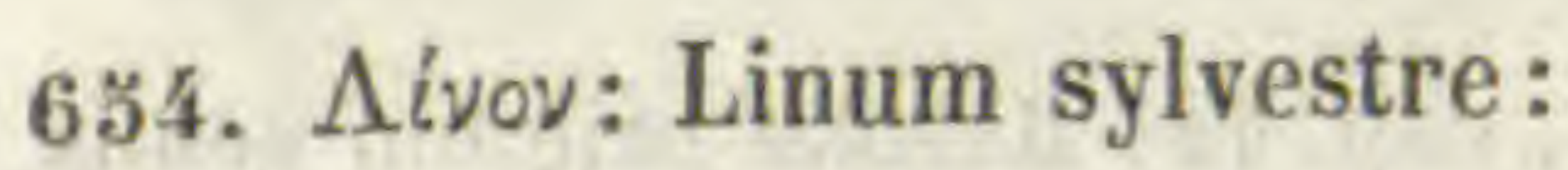

Specimen fructiferum. Linum tenuifolium Linn.

655. Linnm sylvestre:

Lib. XIV, cap. 35 .

Specimen floriferum. Linum gallicum Linn.

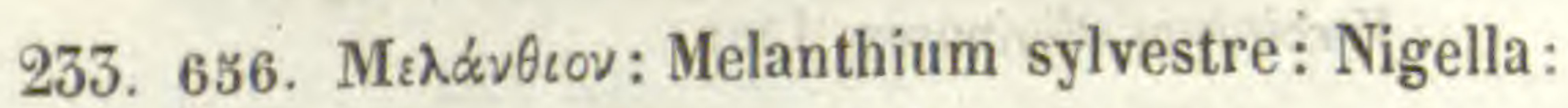

Lib. XV, cap. 11 : Nigella prima.

Specimen floriferum. Nigella damascena Linn.

657. Herba Paris:

Lib. XV, cap. 13.

Specimen floriferum. Paris quadrifolia Linn. 
658. Mínov pord́s: Papaver rhoeas: Rosolaccio:

Lib. XV, cap. 10: Pap. Erraticum primum. Specimen fertile. Papaver Rhaeas Linn.

659. Oxys. Pli : Alleluya :

\section{Lib. XV, cap. 2.}

Specimen floriferum et fructiferum. Oxalis corniculata Linn.

234. 660. Ki $i \theta_{0}$ : Cisthus fæmina:

Lib. XV, cap. 15.

Specimen floriferum. Cistus salvifolius Linn.

661. Kiatos: Cisthus mas:

Lib. XV, cap. 15.

Specimen floriferum. Cistus incanus Linn.

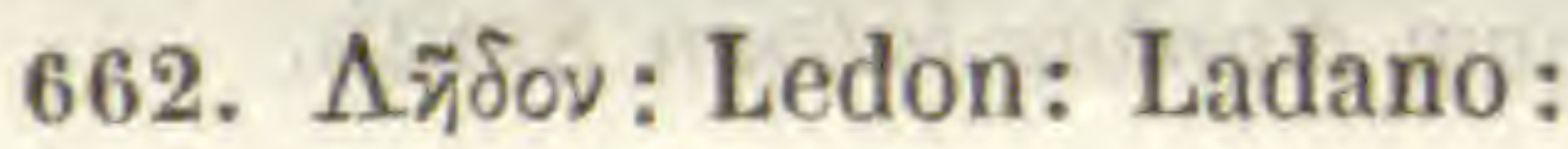

Lib. XV, cap. 16.

Specimen fructiferum. Cistus monspeliensis Linn.

663. Mampè:

Lib. V, cap. 14: Bagolæ primum genus.

Specimen sterile, ut etiam sequens. Vaccinium Myrtillus Linn.

664. Mampè :

Lib. V, cap. 14: Bagolæ alterum genus.

Vaccinium uliginosum Linn.

665. Kvéwpov: Cneorum candidum. Theoph: Casia Vergilii :

Lib. XV, cap. 17: Rhododendron Alpina.

Specimen cum floribus et fructibus. Rhododendron ferrugineum Linn. 
235. 666. Medion: Rapunculus: Raperonzo:

Lib. IX, cap. 28: Rapunculus primus. Specimen floriferum. Campanula Rapunculus Linn.

667. Mr̉jotov: Medion:

Lib. IX, cap. 30: Phyteuma primum. Specimen floriferum. Campanula persicifolia Linn.

668. Medion :

Lib. IX, cap. 30: Phyteuma tertium. Specimen floriferum. Phyteuma Michelii All.

236. 669. Medion: Arch' angelica:

Lib. IX, cap. 30: Phyteuma quartum.

Omnino deest. Videtur Phyteuma Halleri All.

670. Medion: Archangelica:

Lib. IX, cap. 29.

Specimen floriferum. Campanula Trachelium Linn.

\section{Medion:}

Lib. IX, cap. 30: Phyteuma secundum. Specimen floriferum, prorsus fere deficiens. Campanula ratundifolia Linn?

237. 672. Argemonia $3^{\mathrm{a}}$. Pli: Gariofilata montana:

$$
\text { Lib. XIV, cap. } 12 .
$$

Flos et folium. Geum montanum Linn.

673. Argemonia 3a. Pli: Gariofilata:

$$
\text { Lib. XIV, cap. } 12 .
$$

Ramus fructifer, cum folio radicali. Geum urbanum Linn.

674. Kinparis: Clematis $2^{\mathrm{a}}$ : Flammula: Viticella:

$$
\text { Lib. XIV, cap. } 4 \text { : Viticella. }
$$

Specimen floriferum. Clemátis Flammula Linn. 
238. 675. 'Oเvd่v日n: Oenanthe:

$$
\text { Lib. XIV, cap. } 14 .
$$

Folium tantum. Spircea Ulmaria Linn.

676. Oenanthe altera: Filipendula :

$$
\text { Lib. XIV, cap. } 13 .
$$

Specimen floriferum. Spircea Filipendula Linn.

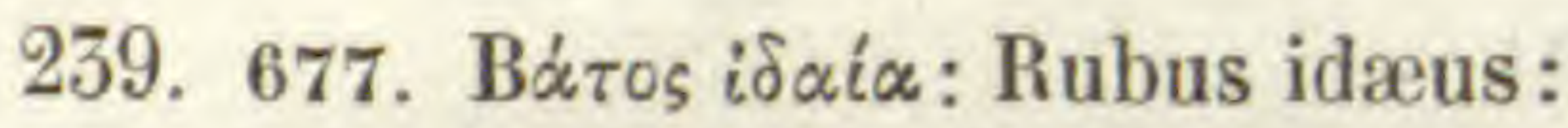

Lib. III, cap. 11: Rubus tertius.

Specimen sterile. Rubus idceus Linn.

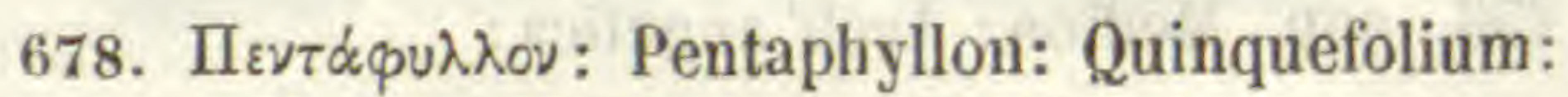

Cinquefoglo:

$$
\text { Lib. XIV, cap. } 19 .
$$

Specimen sterile, mancum. Potentilla reptans Linn.

679. Heptaphyllon alterum :

Lib. XIV, cap. 20: Heptaphyllon primum. Specimen floriferum. Potentilla hirta Linn. et auct.

680. Heptaphyllon. Hermolai: Tormentilla:

Lib. XIV, cap. 21: Tormentilla prima.

Specimen floriferum. Potentilla Tormentilla Sibth.

240. 681. Heptaphyllon magnum:

Folia duo. Polentilla recta Linn. et auct.

682. Heptaphyllon candidum:

Lib. XIV, cap. 20: Heptaphyllon alterum. Specimen floriferum. Potentilla caulescens Linn.

683. Sanicula :

$$
\text { Lib. XIV, cap. } 22 .
$$

Specimen floriferùm. Sanicula europcea Linn. 
684. Boútopos: Butomus fæmina. Theoph :

Lib. XIV, cap. 17.

Umbella florifera, cum folio. Butomus umbellatus Linn.

241. 685. Butomus fæmina altera: Plantago aquatica:

Lib. XIV, cap. 15.

Specimen floriferum. Alisma Plantago Linn.

686. Boútopos: Butomus mas: Barba sylvana:

Lib. XIV, cap. 16.

Specimen floriferum. Sagittaria sagittifolia Linn.

687. Plantago aquatica parva:

Lib. XIV, cap. 15.

Specimen floriferum et fructiferum. Damasonium stellatum Pers.

242. 688. 'A $\lambda x_{\varepsilon}^{\prime} \alpha$ : Alcea:

Lib. XIV, cap. 33.

Specimen floriferum et fructiferum. Lavatera punclata All.

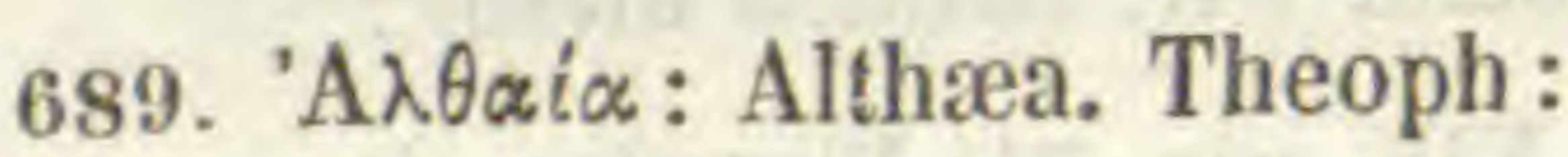

Lib. XV, cap. 18.

Specimen floriferum. Abutilon Avicenna Gærtn.

690. K $\alpha v v \alpha \beta \iota s \dot{\alpha} \gamma p i \alpha$ : Cannabis sylvestris : $p$ Alcea :

Lib. XIV, cap. 34: Alcea altera.

Specimen floriferum. Althcea cannabina Linn.

245. 691. 'A $\lambda$ aía: Althrea. Diosc: Bismalva: Malvavischio:

Lib. XIV, cap. 31: Malva sylvestris Vismalva appellata.

Specimen floriferum. Althcea officinalis Linn. 
692. Malope. Pli : Malvone:

Lib. XIV, cap. 30 .

Specimen floriferum. Althcea rosea Cav.

693. Malope. Pli : Malvone:

Lib. XIV, cap. 30.

Specimen floriferum et fructiferum. Althcea ficifolia Cav.

244. 694. Malva sylvestris:

Lib. XIV, cap. 34: Alcea parva.

Specimen floriferum. Malva moschata Linn.

695. M $\alpha \lambda \dot{\alpha} \chi \eta$ : Malache: Malva domestica candida:

Lib. XIV, cap. 30.

Specimen floriferum. Malva sylvestris Linn.

696. Guadarella de Tintơri :

Lib. IX, cap. 3 .

Specimen floriferum. Reseda Luteola Linn.

245. 697. Aristalthæa. Pli: Althæa maior :

Lib. XIV, cap. 3I: Vismalva altera.

Specimen floriferum. Hibiscus roseus Thore.

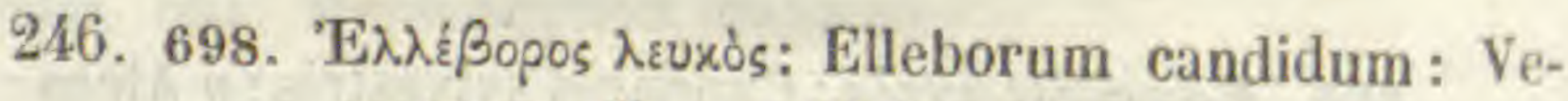
ratrum album: Elabro bianco:

Lib. XV, cap. 27: Elleborum album alterum. Specimen floriferum. Veratrum nigrum Linn.

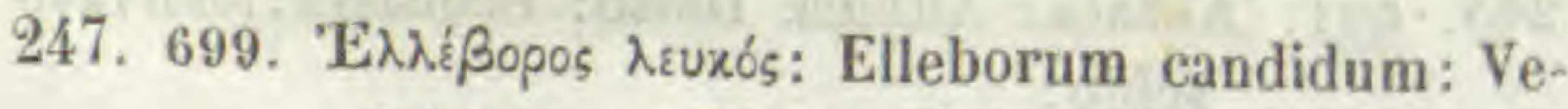
ratrum candidum : Elabro bianco:

Lib. XV, cap. 27: Elleborum album primum. Specimen floriferum. Veratrum album Linn. 


\section{$-111-$}

700. 'E $\mathrm{\lambda} \lambda \hat{\varepsilon} \beta$ opos $\mu \dot{\varepsilon} \lambda \alpha s$ : Elleborum nigrum: Veratrum nigrum: Euneaphyllon. Pli: Elabro nero:

Lib. XV, cap. 29.

Specimen floriferum. Helleborus foetidus Linn.

248. 701. Elleborum nigrum: Consiligo. Pli : Elabro nero:

Lib. XV, cap. 28: Elleborum nigrum primum. Flos et folium Hellebori viridis Linn.

702. 'E $\lambda \lambda \varepsilon \dot{\varepsilon} \beta$ opos $\mu_{\varepsilon}^{\prime} \lambda \alpha_{s}$ : Elleborum nigrum. Diosc:

Lib. XV, cap. 28: Elleborum nigrum alterum.

Flos Hellebori nigri Linn.

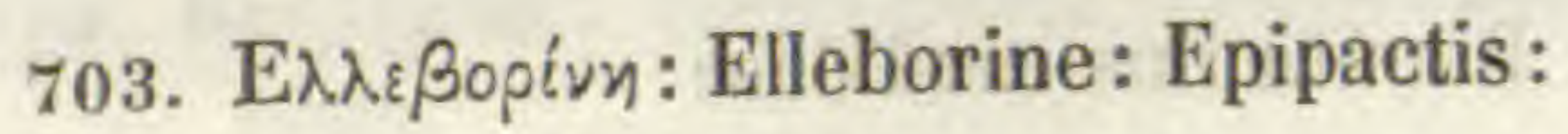

Lib. XV, cap. 30.

Folia duo Eranthis hyemalis Salisb.

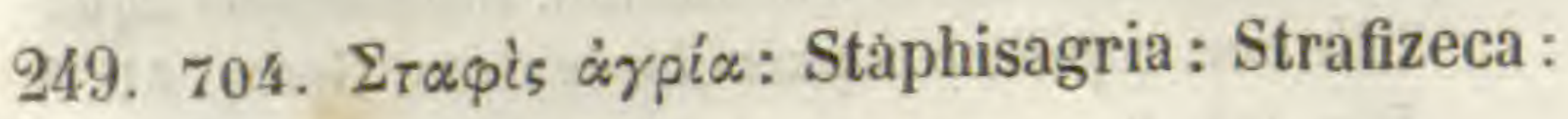

Lib. XV, cap. 31.

Specimen floriferum. Delphinium Staphisagria Linn.

705. Aconitum : Lycochthonon: Napello:

Lib. XV, cap. 32: Napelli primum genus?

Fragmenta folii tantum exstantia, ad Delphinii speciem, ut videtur D. velutinum Bert., pertinentia.

706. Aconitum: Lycochthonon: Napello:

Lib. XV, cap. 32: Napelli secundum genus.

Folium tantum. Aconitum Lycoctonum Linn.

250. 707. Aconitum: Lycochthonon: Luparia: Vulparia:

Lib. XV, eap. 34: Vulparia prima.

Specimen fructiferum. Trollius europceus Linn. 
708. Pulsatilla:

Lib. XIV, cap. 11.

Specimen fructiferum. Anemone alpina Linn., var. quæ $A$, millefoliata Bert.

709. B $\propto \tau \rho \alpha \chi<$ เov: Batrachion: Ranunculus $2^{\mathrm{s}}$ : Herba Sardoa: Apium risus:

Lib. XIV, cap. 5: Ranunculi genus montanum primum.

Specimen fertile. Anemone narcissiflora Linn.

251. 710. Sardoa altera: Ranunculus candidus:

Lib. XIV, cap. 5: Ranunculi genus montanum tertium.

Specimen floriferum. Ranunculus aconitifolius Linn.

711. Ranunculus candidus:

Lib. XIV, cap. 2: Ranunculus in aquis proveniens, foliis in capillaceam tenuitatem scissis. Specimen floriferum et fructiferum. Ranunculus aquatilis var. capillaceus parviflorus Coss. Germ. Atl. fl. Par. tab. 2.

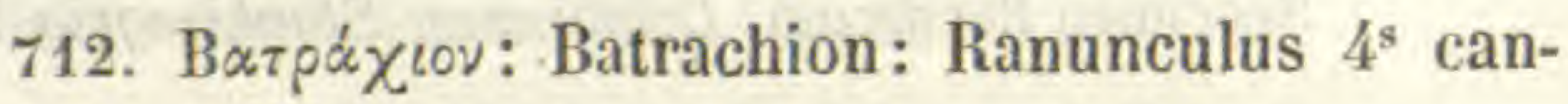
didus:

Lib. XIV, cap. 2: Ranunculus in aquis proveniens, foliis utriusque generis.

Specimen ut præcedens. Ranunculus aquatilis var. heterophyllus grandiflorus Coss. Germ. 1. c.

252. 713. Chelidonium montanum:

Lib. XV, cap. 34: Vulparia altera.

Specimen fructiferum. Caltha palustris Linn.

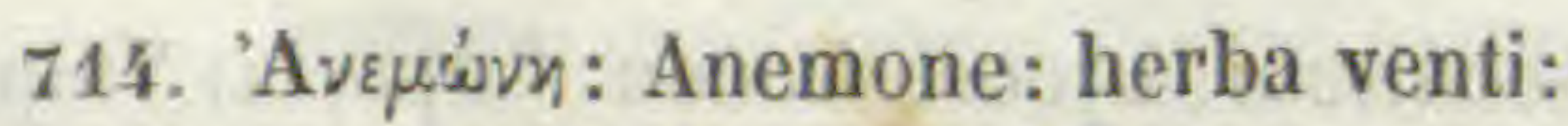

Lib. XIV, cap. 9: Anemone prima.

Specimen floriferum. Anemone hortensis Linn. 


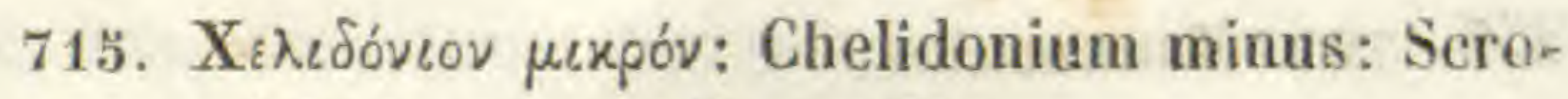
fularia minor: Favagello:

Lib. XIV, cap. 7.

Specimen floriferum. Ranunculus Ficaria Linn.

716. Anemone candida:

Lib. XIV, cap. 10.

Specimen fertile. Anemone nemorosa Linn.

255. 717. Ranunculus:

Lib. XIV, cap. 2: Ranunculus semine hirsuto alter foliis multifidis.

Specimen floriferum et fructiferum. Ranunculus arvensis Linn.

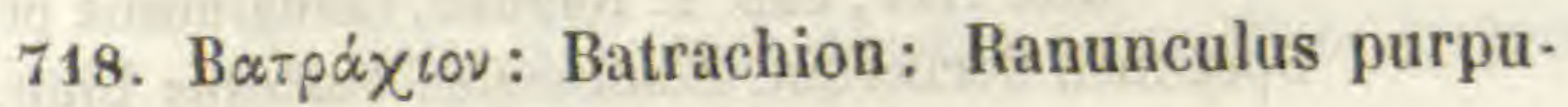
reus :

Lib. XIV, cap. 6.

Specimen floriferum. Adonis autumnalis Linn.

719. Ranunculus:

Lib. XIV, cap. 2: Ranunculus primus.

Specimen floriferum et fructiferum. Ranunculus sceleratus Linn.

254. 720. B $\alpha \tau p \dot{\alpha} \chi\left\llcorner 0 v:\right.$ Batrachion: Ranunculus $3^{\text {us }}$ :

Lib. XIV, cap. 2: Ranunculus semine hirsuto primus folio subrotundo.

Specimen floriferum et fructiferum. Ranunculus muricatus Linn.

721. Ranunculus:

Lib. XIV, cap. k: Ranunculi genus in paludibus, folio Plantaginis.

Specimen fructiferum. Ranunculus ophioglossifolius Vill. 
255. 722. Ranunculus:

Lib. XIV, cap. 4: Ranunculi genus in paludibus, folio Coronopi.

Specimen floriferum. Ranunculus Flammula Linn.

723. Ranunculus: Chrysanthemum. Democriti :

Lib. XIV, cap. 3.

Specimen floriferum. Banunculus repens Linn.

724. Ranunculus:

Specimen floriferum. Ranunculus Philonotis Retz.

256. 725. Ranunculus $p^{s}$ : Ranunculus rapaceus: Pie corvino:

Lib. XIV, cap. 2: Ranunc. lanuginosus primus. Specimen floriferum. Ranunculus bulbosus Linn.

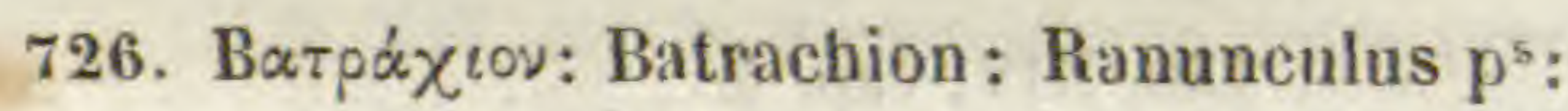

Lib. XIV, cap. 2: Ranunc. lanuginosus alter. Specimen floriferum et fructiferum. Ranunculus velutinus Ten!

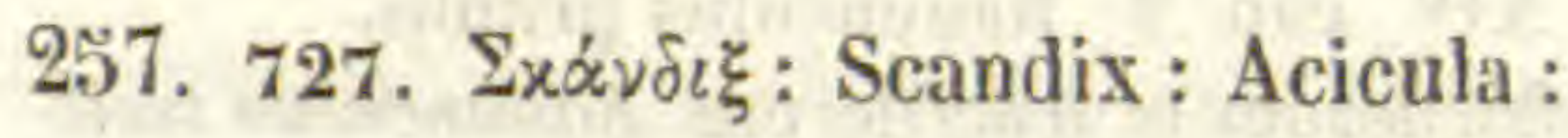

Lib. VII, cap. 24.

Specimen floriferum et fructiferurn. Scandix Pecten Veneris Linn.

728. Pecten veneris. Pli : Acus muscata: Herba moscada :

Lib. XIV, cap. 29.

Specimen floriferum. Erodium cicutarium L'Hér.

729. $\Sigma_{\iota} \delta$ ирiтьs: Sideritis $3^{\mathrm{a}}$ : Herba Roberti: Robertiana:

Lib. XIV, cap. 28.

Specimen fructiferum. Geranium robertianum Linn. 
258. 730. Geranium: Pes columbinus minor:

Lib. XIV, cap. 25 ,

Omnino deest. An Geranium columbinum Linn?

731. Geranium balsaminum :

Lib. XIV, cap. 27.

Specimen fructiferum. Geranium sylvaticum Linn.

732. Geranium:

Lib. XIV, cap. 25.

Specimen floriferum et fructiferum. Geranium dissectum Linn.

733. Geranium: Pes columbinus maior: Pie columbino:

Lib. XIV, cap. 26: Pes Columbinus hederæfolius.

Specimen fructiferum. Geranium nodosum Linn.

734. Гвра́viov: Geranium 2um. Diose: Rostrum gruis :

Lib. XIV, cap. 25: Pes Columbinus tertius. Specimen floriferum et fructiferum. Erodium malacoides Willd.

259. 735. Ilatovia: Pæonia mas:

Lib. XV, cap. 36.

Specimen floriferum, sed valde mancum. Pceonia corallina Retz.

736. Uatovía : Pronia fremina:

Lib. XV, cap. 36 .

Pars folii tantum. Pceonia peregrina Mill.

737. Dittamo bianco: Frassinello:

Lib. XV, cap. 37.

Itidem pars folii. Dictamnus albus Linn. 


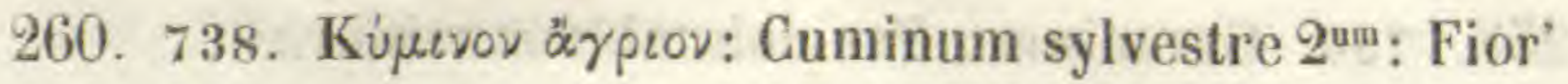
Capucci :

Lib. VI, cap. 69.

Specimen floriferum. Delphinium Ajacis Linn.

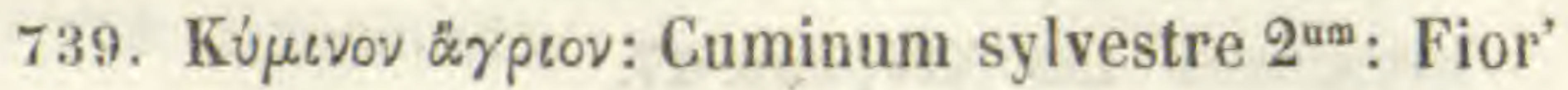
capucci :

Lib. VI, cap. 69: Floris qui vulgo Fior Capuccio alterum genus.

Specimen floriferum. Delphinium Consolida Linn.

740. Aquilegia :

Lib. XV, cap. 38.

Specimen floriferum. Aquilegia vulgaris Linn.

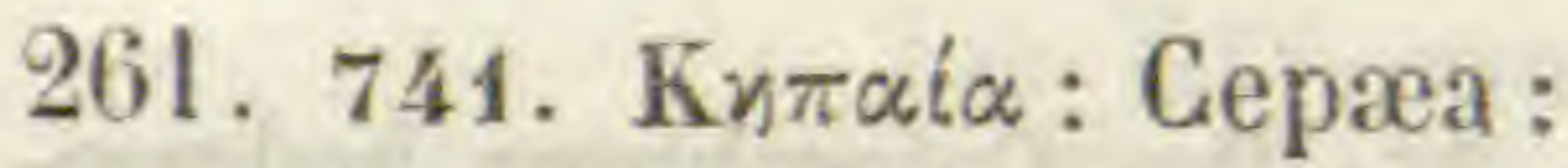

Lib. XV, cap. 23: Cepæa altera.

Specimen sterile. Sedum Anacampseros Linn.

Hoc specimen et plurima sequentia plantarum crassarum valde læsa sunt.

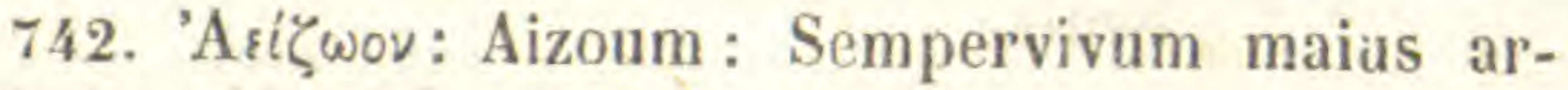
borescens : Sedum :

Lib. XV, cap. 20: Sempervivum alterum.

Ut videtur Sempervivum arboreum Linn.

743. Sempervivum minimum :

Lib. XV, cap. 24: Paronychia secunda?

Sedum dasyphyllum Linn?

744. 'A Lib. XV, cap. 22.

Sedum boloniense Lois.

745. Sempervivum minus arborescens:

Lib. IV, cap. 13: Frutex in marittimis asperis Cassiæ ligneæ similis.

Sucda fruticosa Forsk. 
262. 746. Cotyledon:

Lib. XV, cap. 24: Paronychia prima.

Sedum stellatum Linn.

747. Cepæa:

Lib. XV, cap. 23: Cepæa itr collibus, flosculis candicantibus.

Sedum Cepcea Linn.

748. Cерæa:

Lib. XV, cap. 23: Cepæa flosculis luteis.

Specimen floriferum. Saxifraga aizoides Linn.

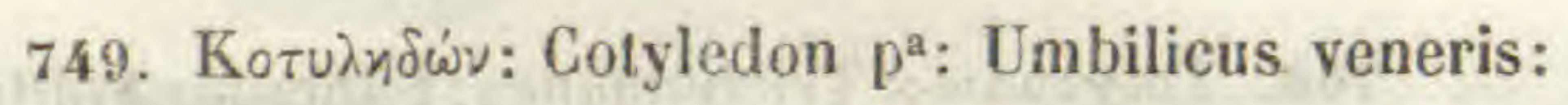

Lib. XV, cap. 25: Umbilicus Veneris primus.

Folia radicalia duo. Umbilicus pendulinus Dec.

750. Koт $u \lambda \eta \delta \dot{\omega} v$ : Cotyledon $2^{\mathrm{a}}$ : Faba inversa: Crassula maior: Fava grassa:

Lib. XV, cap. 26: Crassula prima.

Specimen mox florendum. Sedum maximum Sut.

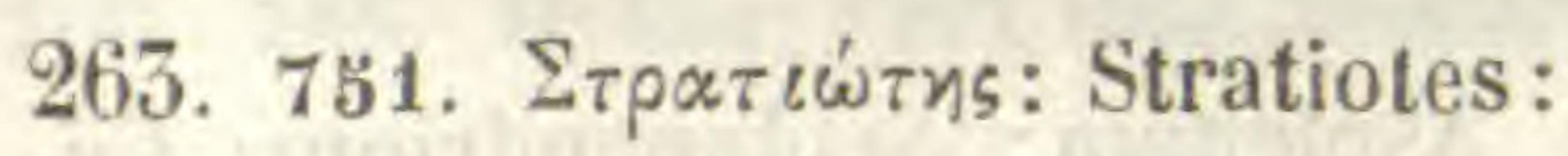

Lib. XVI, cap. 36 .

Specimen sterile. Salvinia natans All.

752. Lenticula palustris:

Lib. XVI, cap. 35 : Lenticulæ alterum genus. Specimen sterile, Lemna trisulca Linn.

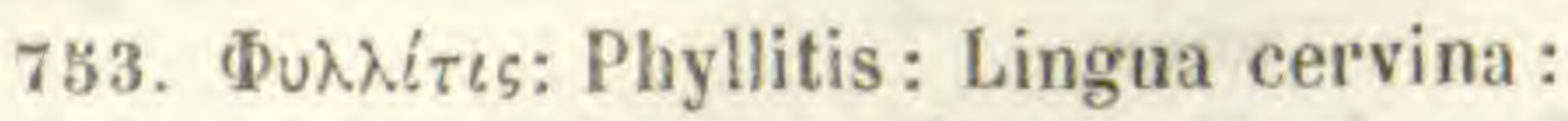

Lib. XVI, cap. 7.

Frons fertilis. Scolopendrium officinarum Swartz.

754. $\Delta$ p

Lib. XVI, cap. 13: Adianti nigri aliud genus. Fragmentum frondis fertilis tantum exstans. Asplenium Adiantum nigrum Linn. 


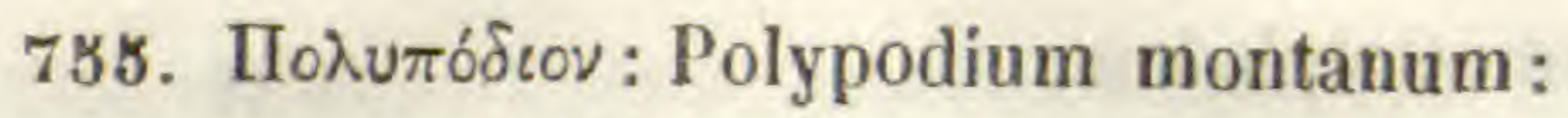

Lib. XVI, cap. 5: Filicis montanæ genus primum.

Frons sterilis. Blechnum Spicant Roth.

264. 756. Saxifragum alterum:

Specimen fertile. Asplenium septentrionale Swartz.

757. 'Adı́xvтov: Adiantum nigrum. Theoph: Saxiphragum. Pli : Ruta muraria :

Lib. XVI, cap. 13: Adiantum nigrum primum. Specimen fertile. Asplenium Ruta muraria Linn.

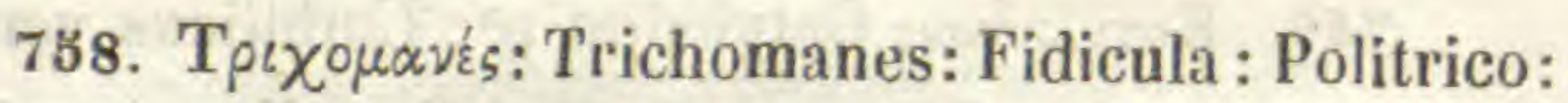

Lib. XVI, cap. 11: Polytricum.

Specimen fertile. Asplenium Trichomanes Linn.

739. 'A S¿dvtov: Adiantum album: Capillus veneris: Capelvenero:

Lib. XVI, cap. 12.

Frons fertilis. Adiantum Capillus Veneris Linn.

760. "A $\sigma \pi \lambda$ иขov: Asplenum: Scolopendrium: Cetrach :

Lib. XVI, cap. 9.

Specimen fertile. Gymnogramme Ceterach Spreng.

265. 761. $\Lambda \circ \gamma \chi \tilde{\imath} \tau \iota \varsigma:$ Lonchitis aspera :

Lib. XVI, cap. 5: Filicis montanæ genus alterum.

Frons fertilis. Aspidium Lonchitis Swartz.

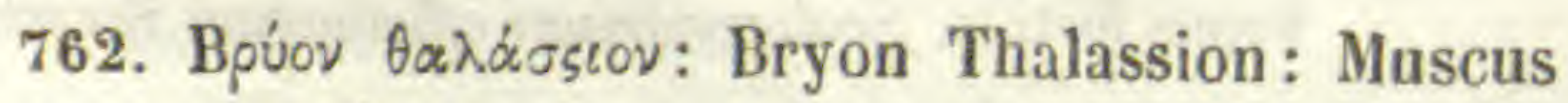
marinus :

Lib. XVI, cap. 24.

Plane fere deficiens. Est Confervacea quædam, verisimiliter Ulva intestinalis. 
763. Alga:

Lib. XVI, cap. 2.

Frustillum folii Posidonice Caulini Kon.

764. Bryon marinum. Pli :

Lib. XVI, cap. 26.

Frons Ulvae.

765. Фúxos $\theta \alpha \lambda \alpha \sigma \varsigma \iota 0 v:$ Fucus marinus latus:

Padina Pavonia Gaill.

766. Фúxos $\theta \alpha \lambda \dot{\alpha} \sigma \varsigma \iota \nu$ : Fucus marinus purpureus: Corallina :

Omnino deest.

767. Фúxos $\theta \alpha \lambda \measuredangle \sigma \varsigma เ o v:$ Phycos marinus candidus: Corallina :

Lib. XVI, cap. 34: Corallina cortice albo punctato.

Est zoophyton, Sertularice species.

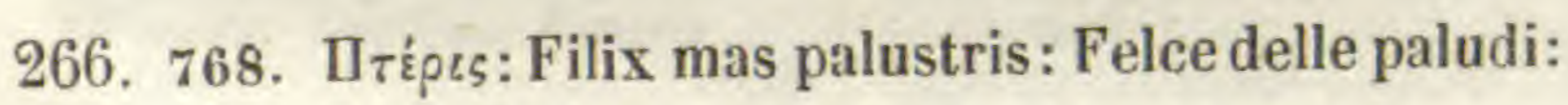

Lib. XVI, cap. 4.

Frons sterilis. Osmunda regalis Linn.

Nus 373 , sub nomine Polygonati multiflori allatus, forsan Polygonato officinali potius referendus. 


\title{
INIDEX
}

\section{NOMINUM RECENTIOHUM PLANTARUM}

\author{
DE QUIBUS IN HOC OPERE AGITUR.
}

Numerus indicat paginam operis.

Abies excelsa Dec. - pectinata Dec. ........ jvi Abutilon Avicennæ Giertn. . 109 Acanthus molis Linn. . . 73 Acer campestre Linn. . . . . 26 - Pseudo-Platanus Linn. . ivi Achillea Ageratum Linn. . . 49 - Millefolium Linn. ..... 51 - Ptarmica Linn. ..... 48 Aconitum Lyeoctonum Linn. 111 Actæa spicata Linn. . . . . 74 AdenosiylesalpinaBluff. Fing. 51 Adiantum Capillus Veneris Linn. . . . . . . . 118 Adonis autumnalis Linn. . . 113 Aegopodium Podagraria Lin. כอ̆ Agrimonia Eupatoria Linn. . 70 Agrostis vulgaris With. . . . 58 Ajuga Chamæpitys Schreb. . 68 - reptans Linn. . . . . . . 605 Alchemilla alpina Linn. ... . 45 - vulgaris Linn. . . . . . . ivi Alisma Plantago Linn. . . . 109 Allium magicum Linn. . . 102

- nigrum Auct. . . . . . . . ivi

- pallens Linn. . . . . . . 101

- roseum Linn. ... . . . . ivi

- triquetrum Linn. . . . . . ivi Alnus glutinosa Garln. ... 26 Althæa cannabina Linn. . . . 109

- ficifolia Cav........ 110

- oflicinalis Linn. . . . . 109

- rosea Cav. ... . . . . 110

Alyssum campestre Linn. . . 92

Amarantus tricolor Linn. . . $\$ 2$

Ammi Visnaga Lam.
Anacyclus radiatus Lois. . . 47 Anagallis arvensis Linn . . . 77 Anagyris feetida Linn. . . . . 30 Anchusa sempervirens Linn. 41 Androsæmum officinale $\mathrm{All}$. 105 Anemone alpina Linn. . . . 112 - Hepatica Linn.. . . . . 104 - hortensis Linn. . . . . 112 - millefoliata Bert... . . . . ivi

- narcissiflora Linn. . . . . ivi - nemorosa Linn. . . . . 113 Anethum graveolens Linn. 55 - segetum Linn. . . . . . . ivi Angelica sylvestris Linn. . . 58 Anthemis Cota Linn. . ... 47 Anthriscus Cerefolium Hoffm. $\quad 37$ Anthyllis Barba Jovis Linn. . 30 - tetraphylla Linn... . . 81 Antirrbinum latifolium Dec.. 85 - Orontium Linn. ..... ivi Apium graveolens Linn. . . . . $\mathbf{5 4}$ Aquilegia vulgaris Linn. . 116 Arabis alpina Linn. . . . . . 95

- hirsuta Scop....... 92

- sagittata Dec. ........ ivi

Arisarum vulgare Targ. . . . 73 Aristolochia Clematitis Linn. $10 \mathrm{~s}$ - rotunda Linn. . . . . . . ivi Armeria elongata. . . . . . 71 Arrhenatherum avenaceum Roem. Schult. ..... 59 Artemisia camphorata Vill. . כ0 - coerulescens Linn. . . . . 49

- Dracunculus Linn. . ... . ivi

- pontica Linn. . . . . . 50

- vulgaris Linn. . . . . . ivi 
Arum italicum Mill.

Asarum europæum Linn.

Asparagus tenuifolius Linn. .

Asperula cynanchica Linn.

- scabra Presl.

Asphodeline lutea Reich.

Asphodelus albus Willd.

Aspidium Lonchitis Swarts. 118

Asplenium Adiantum nigrum

$$
\text { Linn. . }
$$

117

- Ruta muraria Linn.

118

- septentrionale Swarlz.

- Trichomanes Linn.

Aster alpinus Linn.

\section{ivi}

ivi

- Tripolium Linn. ..... 52

Asteriseus spinosus Gr. God. 47

Atriplex Halimus Linn. . . . 44

Atropa Belladonna Linn. . . 74

Ballota fotida Lam. . . . . 64

- Pseudodictamnus Benth. . 6 వ

Barbarea vulgaris $R$. Br. . 91

Bartsia viscosa $\operatorname{Linn}$. . . . 86

Berberis vulgaris Linn. . . $\quad 31$

Betonica officinalis Linn. . . 64

Biarum tenuifolium Schott. . 73

Bidens tripartita Linn. . . 52

Blechnum Spieant Roth. . 118

Bonaveria Securidaca Scop. . 81

Botryanthus odorus Kunth. . 99

Brassica nigra Koch. .... 91

Briza media Linn. . . . . . . 58

Bromus sterilis Linn. . . . . ivi

Brunella alba Pall. . . . . 65

- vulgaris Linn. . . . . . . ivi

Bryonia dioica Jacq. . . . 74

Bunium Bulbocastanum Linn. 37

Bupleurum Gerardi Jacq. ... 55

- protractum Link et Hoffm. ivi

Butomus umbellatus Linn. . 109

Buxus sempervirens Linn. . 32

Cakile maritima $S c o p . . . . .91$

Calamintha ClinopodiumBent. 64

- grandiflora Moench. . . 66

- officinalis Møench. ... ivi

Caltba palustris Linn. . . . . 112

Campanula persicifolia Linn. 107

- Rapunculus Linn.

- rotundifolia Linn.

ivi

- Trachelium Linn.

ivi

ivi

Canna indica Linn.

101

Cannabis sativa Linn.

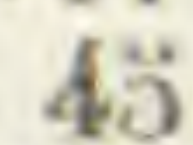

Capparis rupestris Sibth. Sm. 105
Cardamine chelidonia Lam. . 95

Cardiospermum Halicacabum Linn. . . . . . . . 104

Carduus nutans Linn. . . . 57

- Personata Jacq. . . . . . ivi

Carex extensa Good. . . . . 60

- muricata Linn. . . . . . 62

- remota Linn. . . . . . 60

Carlina acanthifolia All. . . . 57

- acaulis Linn. . . . . . . ivi

- corymbosa Linn. . . . . . 55

Carthamus tinctorius Linn. . 56

Celtis australis Linn. . . . . 35

Centaurea alba Linn. . . . . 58

- Calcitrapa Linn. . . . . ธ ธั5

- nigrescens Willd. . ... 57

- solstitialis Linn. . . . . . รั5

- sphærocephala Linn. . 5 56

Centranthus ruber Dec. . . . 59

Cephalanthera ensifolia Rich. 102

- pallens Rich. . . . . . . . ivi

Cephalaria transylvanica Sch. $\quad 69$

Cerastium arvense Linn. . . 77

- glomeratum Thuill. ... ivi

Ceratonia Siliqua Linn. . . 30

Cerinthe aspera Roth. . . . . 41

Chærophyllum magellense $T . \quad \mathbf{3 7}$

Chelidonium majus Linn. . . 88

Chenopodium Botrys Linn. . 42

- ficifolium Smith. ..... 44

- murale Linn. ..... . ivi

- Vulvaria Linn. ... . . . jvi

Chlora perfoliata Linn. . . . 88

Chondrilla juncea Linn. . . . 54

Chrysanthemum coronarium

- Linn.......... 46

- Myconis Linn. ... . . . 48

- segetum Linn. . . . . . 47

Gircæa lutetiana Linn. . .... 95

Cirsium lanceolatum Scop. , 57

- monspessulanum All. . . ivi

Cistus incanus Linn. . . . 106

- monspeliensis Linn. . . . ivi

- salvifolius Linn. ..... ivi

Llematis Flammula Linn. . . 107

Cochlearia Armoracia Linn, . 92

Colchicum autumnale Linn. . 100

Colocasia antiquorum Scholt. 73

Colutea arborescens Linn. . $\mathbf{3 0}$

Conium maculatum Linn. . : 37

Convallaria majalis Linn. . . 72

Convolvulus arvensís Linn. . 90

- Cantabrica Linn. . . . . . ivi

- Cneorum Linn. . . . . . . . ivi 
Convolvulus sepium Linn. . . 90 - Soldanella Linn. . . . . . ivi Coronilla varia Linn. . . . . 82 Corrigiola litoralis Linn... 42 Corydalis cava Schw. Koert. 87 - ochroleuca Koch. . . . 88 Corylus Avellana Linn. .... 25 Cratægus Oxyacantha Linn.. 31 - Pyracantha Pers. . .... ivi Critlimum maritimum Linn. 39 Crocus vernus $A l l \ldots \ldots 101$ Grozophoratinctoria Ad. Juss. 96 Cuscuta Epithymum Linn. . 69 Cyclamen repandum Sibth. Smith......... 104 - vernum Gay......... . ivi Cynodon Dactylon Pers. . . . 60 Cynoglossum pictum Ait. . . Cyperus esculentus Linn. . . - longus Linn.

- olivaris. Targ.

- rotundus. Linn.

- syriacus Parl.

Cylisus Laburnum Linn. . . 50

- spinosus Lam. . . . . . . 28

Damasonium stellatum Pers. 109 Daphne Gnidium Linn. . . . 71

- Laureola Linn. . . . . . . ivi

- Mezereum Linn. . . . . . ivi

Datura fastuosa Linn. . . . . 74

Daucus Carota Linn. ..... 56

Delphinium Ajacis Linn. . . 116

- Consolida Linn. . . . . . ivi

- Staphisagria Linn. . . . . 111

- velutinum Bert. . . . . . ivi

Dentaria bulhifera Linn.. . . 93

- pinnata Lam. . . . . . ivi Deschampsia flexuosa Trin. 58 Dianthus Carthusianorum $L .76$ Dictamnus albus Linn. . . . 115 Digitalis Jutea Linn... . . . 84 Diospyros Lotus Linn..... . 26 Dipsacus sylvestris Mill. . . อั Dorycnium herbaceun Vill. . 80 Dracunculus vulgaris Sehott. 75

Ecbalium Elaterium Rich... . 93 Echinophora spinosa Linn. . 39 Echinops sphrorocephalus $L$. Echium italicum Linn. - plantagineum Linn. Epilobium hirsutum Linn. - montanum Linn.
Epilobium spicatum Lam. . .

- tetragonum Linn. . . . . ivi

Equisetum arvense Linn. . . 46

- ramosum Schleich. .... ivi

- Telmateja Ehrh,..... ivi

Eragrostis megastachya Lin. 60

Eranthis hyemalis Salisb. . . 111

Erica arborea Linn. . . . . , 28

- carnea Linn. . . . . . . . 27

- scoparia Linn. . . . . . . ivi

Erigeron acre Linn. . . . . . 53

Erodium eicutarium L'Her. . 114

- malacoides Willd. .... 115

Eryngium amethystinum Lin. $\quad 56$

- campestre Linn. . . . . . ivi

- maritimum Linn.. . . ... ivi

Erythræa Centaurium Pers. . 88

Eupatorium cannabinum Lín. 52

Euphorbia amygdaloides Lin. 97

- Chamæsyce Linn. . . . . ivi

- Characias Linn. . . . . 96

- Cyparissias Linn. .... ivi

- dendroides Linn. . . . ivi

- dulcis Linn. . . . . . . 97

- exigua Linn. . . . . . . . ivi

- falcata Linn... . . . . . 98

- Lathyris Linn. . . . . . . 97

- nicæensis All. ...... ivi

- palustris Linn. . . . . . ivi

- Paralios Linn. . . . . . . ivi

- Peplis Linn. . . . . . . . 98

- Peplus Linn. . . . . . . . ivi

- Pithyusa Linn. . . . . . 96

— pubescens Desf. . . . . 97

- spinosa Linn. . . . . 98

Euphrasia officinalis Linn. 86. 87 Evonymus europæus Linn. . 25

Fagopyrum emarginatum Meis. 44 Fagus sylvatica Linn...... 25 Farsetia clypeata $R$. Br... 93

Ferula Ferulago Linn. . . . . 54

- geniculata Guss. . . . . ivi

Filago gallica Linn. . . . . 50

- germanica Linn. ..... ivi

Fraxinus Ornus Linn. . . . 26

Galega officinalis Linn. ... 81 Galeopsis angustifolia Ehrh. 65 Galium Cruciata Scop. . . 9 94

- Iævigatum Linn. . . . . . ivi

- lucidum All. . . . . . . . ivi

- Mollugo Linn. . . . . . ivi ivi

- verum Linn. . . . . . . ivi 
Genista eandicans Linn.

- germanica Linn.

- pilosa Linn.

- radiata Scop.

- tinctoria Linn.

Gentiana asclepiadea linn.

- campestris Linn.

- Cruciata Linn.

- Iutea Linn.

Geranium columbinum Linn. 115

- dissectum Linn.

- nodosum Linn.

- robertianum Linn.

- sylvaticum Linn.

Geum montanum Linn.

-- urbanum Linn.

Gladiolus segetum Gawl. 100

Glancium luteum Scop. ... 87

Globularia incanescens Viv . 70

Gnaphalium luteo-album L. 5 ?

- rectum Smith.

- sylvaticum Linn.

51

ivi

Gratiola officinalis Linn. . . 88

Gymnogramme Ceterach Spr. 118

Hedysarum coronarium Lin. 81 Helianthemum Fumana Mill. 104 - vulgare Gartn........ ivi Helichrysum angustifolium Dec.

Heliotropium europæum Lin. Helleborus fœtidus Linn.

- niger Linn.

- viridis Linn.

Helminthia echioides Gertn. Helosciadium nodiflorum $\boldsymbol{K}$. Hemerocallis fulva Linn. . . Heracleum Pollinianum Bert. Hermodactylus tuberosus Sal. 100 Herniaria hirsuta Linn. Hibiscus rosens Thore. Hieracium elatum Fries.

- Pilosella Linn.

- piloselloides Vill.

- prenanthoides Vill. 42. 45

110

54

53

ivi

54

Hippophaë rbamnoides Linn.

Holeus lanatus Linn.

Hordeum murisum Linn. . . jvi

Hottonia palustris Linn. . . . 77

Humnlus Lapulus Linn. . . . 45

Hydrocharis Morsus ranæ $L .10 \mathrm{~s}$

Hyoscyamus albus Linn.

- niger Linn.

Hypericum Coris Linn.

30

vi

马!

40

ivi

ivi

54

56

98 4

9

79

vi

vi

vi

vi

vi

4

13

i

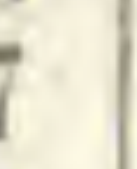

0

\section{7}

- Scariola Linn.. . . . . . .

Lagurus ovatus Linn. . . . 60

Lamium Galeobdolon Crants. 65

- maculatum Linn. . . . . 64

Lappa minor Dec. . . . . . 58

Laserpitium latifolium Linn. $\mathbf{5 4}$

- Siler Linn. . . . . . . . 55

Lathyrus latifolius Linn. . 82

Lavandula Stœechas Linn. . . 69

Lavatera punctata All. . . 109

Lemna trisulca Linn. . . . . 117

Leontice Leontopetalon Linn. 88

Leonurus Cardiaca Linn. . . 63

Leopoldia comosa Parl.. . . 99

Lepidium Draba Linn... . . . 92

- graminifolium Linn. . . . 93 
Lepidium latifolium Linn.

- sativum Linn.

Lepigonum rubrum Wahlb.

Leucanthemum vulgare $L a m$.

Leucojum æstivum Linn.

Levisticum officinale Koch.

Ligustrum vulgare Linn.

Lilium bulbiferum Linn.

- croceum Chaix.

- Martagon Linn.

Linaria minor Desf.

- purpurea Mill.

- spuria Mill.

- vulgaris Mill. .

Linosyris vulgaris Cass.

Linum gallicum Linn.

- tenuifolium Linn.

Listera ovata $R$. Brown.

Lithospermun arvense Linn. . 41 officinale Linn.

Lolium italicum Al. Braun.

Lonicera Caprifolium Linn. .

Lotus corniculatus Linn.

- hirsutus Linn.

Lunaria rediviva Linn. . . .

Lupinus angustifolius Linn.

Luzula nivea Dec.

Lychnis coronaria Lam.

- diurna Sibth.

- Githago Lam.

- vespertina Sibth.

Lycium europæum Linn.

- mediterraneum Dun... . .

Lycopodium annotinum Linn.

Lycopus europæus Linn.

Lysimachia Nummularia Lin.

- vulgaris Lirn.

Lythrum Salicaria Linn.

Malva moschata Linn.

110

- sylvestris Linn.

ivi

Mandragora microcarpa Bert.

- officinarum Linn.

- vernalis Bert.

73

ivi

ivi

Marrubium vulgare Linn,... 65

Marsdenia erecta $R . B r \ldots .89$

Matricaria Chamomilla Linn.

Medicago falcata Linn.

- sativa Linn.

47

80

79

Melampyrum arvense Linn. . 86

Melia Azedarach Linn. . . . . 50

Melilotus alba Desr. . . . 79. 80

Melissa officinalis Linn. . . 63

Melittis Melissophyllum Lin. ivi
Mentha aquatica Linn:

- Pulegium Linn.

- sylvestris Linn.

Mercurialis annua Linn. . . 90.91

- perennis Linn. . . . . . 91

Meum athamanticum Jaeq. . 55

Micromeria juliana Benth... 67

Momordica Balsamina Linn. 95

Muscari comosum Mill. . . . 99

Myosotis palustris With. . 40

Myriophyllum verticillatum $L .64$

Myrrhis odorata Scop. . . 37

Myrtus communis Linn. . . . $\mathbf{3 1}$

Narcissus poeticus Linn. . . 99

- Tazetta Linn. . . . . . . . . ivi

Nasturtium anceps Dec..... 91

Nepeta Cataria Linn. . . . . 66

- Glechoma Benth. ..... 65

Nerium Oleander Linn. . . . 89

Nigella damascena Linn. . . 105

Nuphar luteum Smith..... ivi

Nymphæa alba Linn. . . . . ivi

Obione portulacoides $\mathrm{Mog}$. . 44

Odontites lutea Reich.. . . . 86

Oenanthe Phellandrium Lam. $\quad 57$

- pimpinelloides Linn. . . . 34

Ononis campestris Koch. Zis. 79

- spinosa Linn. ...... ivi

Onopordum Acanthium Linn. 55

Ophioglossum vulgatum $\mathrm{Lin} . \quad 75$

Opopanax Chironium Koch. 54

Orchis laxiflora Lam. ..... 102

- maculata Linn....... ivi

Origanum creticum Savi. . . 68

- Dictamnus Linn. . . . . . ivi

- hirtum Benth. ...... ivi

- Majorana Linn. . . . . . 67

- Onites Linn. . . . . . . . ivi

- vulgare Linn. . . . . . 68

Ornithogalum narbonense $L .101$

- pyrenaicum Linn. ..... ivi

- umbellatum Linn, , . . . ivi

Orobanche minor Sult. . . . 86

Orobus niger Linn. . . . . 81

- variegatus Ten. .... 82

Osmunda regalis Linn. . . 119

Ostrya carpinifolia Scop. .. 25

Oxalis corniculata Linn. . 106

Padina Pavonia Gaill. . . . . 119

Preonia corallina Retz.... 115

- peregrina Mill...... ivi 
Pancratium maritimum Linn. 99

Panicum Crus galli Linn. . . 60

Papaver Rhoas Linn. . . . 106

Paris quadrifolia Linn, ... 105

Parnassia palustris Linn. . 105

Passerina hirsuta Linn. . . 28

Pastinaca sativa Linn. . 35. 56

Pedicularis foliosa $\operatorname{Linn} . . .85$

Periploca græeca Linn. . . 89

Petasites officinalis Mcench. . 51

Peucedanum Cervaria Cuss.. $\mathbf{5 8}$

- officinale Linn. . . . . .

- Oreoselinum Moench... 38

- palustre Moench. . . . . ivi

- sulcatum Car. . . . . . 37

- venetum Koch. . . . . . . $\mathbf{3 8}$

Phagnalon sordidum Dec. . . 50

Phalangium Liliago Schreb. . 98

- ramosum Lam. . . . . . . ivi

Pharbitis Nil Chois. ..... 90

Philadelphus coronarius Lin. $\overline{31}$

Phleum asperum Jacq. . . . 60

Phlomis fruticosa Linn. . . . 63

Phoenix dactylifera Linn. . . 28

Phyllirea media Linn. . . . . 29

Physalis Alkekengi Linn. . . 74

- Somnifera Linn.

Pbyleuma Halleri All.

ivi

- Michelíi All.

107

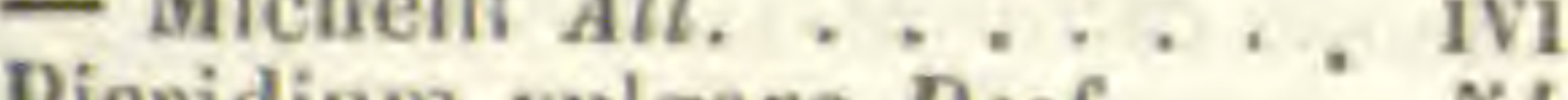

Pimpinella valgare Desf..... 54

Pimpinella Anisum Linn. . . 36

- peregrina Linn. ..... i. ivi

- saxifraga Linn.. . . . . . 37

Pistacia Lentiscus Linn. . . . 29

- Terebinthus Linn. ..... ivi

Plantago arenaria Waldst.Kit. 78

- Bellardi All.

- Coronopus Linn.

- lanceolata Linn.

- major Linn.

Platanthera bifolia Rich. . 102

Plumbago europæa Linn. .. 42

Poa trivialis Linn...... 58

Polyearpon tetraphyllum Lin. 41

Polygala flavescens Dec. . . . 84

— nicrensis Koch. . . . . . . ivi

- vulgaris Dec. . . . . . 8 a

Polygonatum multiflorum. 72. 119

- officinale All.

- verticillatum All.

72

Polygonum aviculare Linn.

- Bistorta Linn.

- Convolvulus Linn.

42

- lapathifolium Linn.
Polygonum maritimum Linn.

- serrulatum Lag. ...... 45

Polytrichum commune Linn. $\quad 46$

Populus alba Linn. . . . . 26

- nigra Linn. . . . . . . 27

Posidonia Caulini Kon. . . 119

Potamogeton natans Linn. 71

Potentilla caulescens Linn. , 108

- hirta Linn. . . . . . . . . ivi

- recta Linn. . . . . . . . ivi

- reptans Linn........ ivi

- Tormentilla Sibth. .... ivi

Prangos ferulacea Lindl. . . 34

Prenanthes purpurea Linn. . 33

Primula Auricula Linn. . . . 78

- suaveolens Bert. . . . . 75

Prunus Laurocerasus Linn. 32

Psoralea bituminosa Linn. . 80

Ptychotis verticillata Dub. . $5 \overline{5}$

Pulicaria dysenterica Gurtn. 48

- odora Reich. . . . . . . . iví

Pulmonaria tuberosa Sehr. 40

Pyrethrum Parthenium $S m$. 149

Pyrus Aria Ehrh. . . . . . 51

- torminalis Ehrh. . . . 27

Pyrola minor Linn. . . . . 9. 94

Ranunculus aconitifolius Lin. $\mathbf{1 1 2}$

- aquatilis Linn. . . . . . . ivi

- arvensis Linn. ...... 115

- bulbosus Linn. . . . . 114

- Ficaria Linn. . . . . . 113

- Flammula Linn. . . . . 114

- muricatus Linn. ...... ivi

- ophioglossifolius Vill. . 113

- Philonotis Retљ. ..... 114

- repens Linn. ........ ivi

- sceleratus Linn. ... . . 113

- velutinus Ten. ...... 114

Raphanus Raphanistrum Lin. 91

Reseda lutea Linn. ..... 104

- Luteola Linn. . .... 110

Rhamnus Alaternus Linn. . . 50

- catbarticus Linn. . . . 26

- Frangula Linn. . . . . . . . ivi

Rhinanthus minor Ehrh. .. 86

Rhododendron ferrugineum Linn. . . . . . . 106

Rhus Coriaria Linn. . . . . 50

- Cotinus Linn. ....... jvi

Ricinus communis Linn. . . 96

Rubus idæus Linn. ..... 108

Bumex Acetosa Linn. . . . 45

- Acetoselia Linn. . . . . 44 
Rumex alpinus Linn.

- obtusifolius Linn.

Ruscus aculeatus Linn.

- Hypoglossum Linn.

- Hypophyllam Linn. . . . . ivi

Sagina procumbens Linn. . . 77

Sagittaria sagittifolia Linn. . 109

Salicornia herbacea Linn. . . 95

Salsola Kali Linn. . . . . . . ivi

- Soda Linn. .......... ivi

Salvia Ftbiopis Linn.. . . . 62

— glutiuosa Linn. . . . . . 65

- lavandulæefolia Vahl. . . 66

- Sclarea Linn.. . . . . . 62

- Verbenaca Linn. . . . . j ji

- virgata Linn. ... . . . . ivi

Salvinia natans $\mathbf{A l l}$.

117

Sambucus Ebulus Linn.... 71

Samolus Valerandi Linn. . . 94

Sanicula europæa Linn. . . . $\mathbf{1 0 8}$

Santolina Chamæcyparissus Linn. .

Saponaria Ocymoides Linn.

- officinalis Linn.

75

ivi

Satureia montana Linn.

Saxifraga aizoides Linn. . . . 117

- Aizoon Linn. . . . . . . . 86

- lingulata Bell. ... . . . . ivi

- rotundifolia Linn. . . . . ivi

Scabiosa columbaria Linn. . 70

- cretica Linn. . . . . . . . ivi

- maritima Linn. . . . . . . ivi

- Succisa Linn. . . . . . ivi

Scandix Pecten Veneris Linn. 114

Scilla bifolia Linn. . . . . . . 99

Scirpus Holoschœenus Linn. . 62

- lacustris Linn. . . . . . . ivi

- maritimus Linn.

Scleranthus annuus Linn. .

Scleropoa rigida Gris.

Scolopendrium officinarum

Swart..........

Scolymus hispanicus Linn.

Scorpiurus subvillosa Linn. .

Scrophularia eanina Linn. . .

- nodosa Linn... .

- peregrina Linn.

Sedum Anacampseros Linn. 116

- boloniense Lois.

- Cepæa Linn.

- dasyphyllum Linn.

- maximum Sut.

- stellatum Linn.
Selaginella denticulata Koch.

Sempervivum arboreum $\boldsymbol{L}$ in. 116

Senebiera Coronopus Poir. 92

Senecio Cineraria Dec. . . 49

- Doria Linn......... 52

- erraticus Bert. . . . . . 49

- laciniatus Bert. . . . ... 50

- saracenicus Linn. .... 52

Serapias Lingua Linn. . . . 102

Serrafalcus mollis Parl.. . . 59

Seseli Bocconi Guss. . . . . 39

Sideritis romana Linn. ... 66

Silene Armeria Linn. . . . 76

- gallica Linn. . . . . . . ivi

- inflata Smith. ....... ivi

- nutans Linn. . . . . . ivi

- Otites Smith. ...... ivi

Sison Amomum Linn. . . . . 38

Sisymbrium Alliaria Scop. . 95

- officinale Scop....... 91

- pinnatifidum Dec. . . . . 92

- polyceratium Linn..... 91

Smilax aspera $\operatorname{Linn.....~} 72$

Smyrnium rotundifolium Mil. 35

Solanum Dulcamara Linn. . 74

- nigrum Linn. . . . . . . 73

- Pseudocapsicum Linn. . 74

Solidago Virgaurea Linn. . 52

Sparganium ramosum Huds. 70

Spartium junceum Linn. . . 79

Specularia Speculum Dec. fil. 103

Spirza Filipendula Linn. . 108

-. Ulmaria Linn. ........ ivi

Stachys annua Linn. . . . 66

- germanica Linn. . . . . 62

- palustris Linn. . . . . . 65

- recta Linn. . . . . . . . 66

- sylvatica $\operatorname{Linn......6.65~}$

Staphylea pinnata Linn.. . . 26

Statice Limonium Linn. . . 45

- oleæfolia Bert. . . . . . ivi

- virgata Willd. ..... ivi

Stellaria Holostea Linn. . . . 77

- media Vill. ......... ivi

- nemorum Linn. ..... jvi

Sticta pulmonaria Ach. ... 45

Streptopus amplexifolius Dec. 71

Styrax officinale $\operatorname{Linn} . . .25$

Suæda fruticosa Forsk. . . 116

Swertia perennis Linn. . . 87

Symphytum officinale Linn. $\quad \$ 1$

Syringa vulgaris Linn. . . 32

Tamarix africana Poir. 
Tamarix gallica Linn.

Tanacetum Balsamita Linn.

- vulgare Linn.

Taxus baccata Linn.

Tetragonolobus siliquosus Moench.

Teucrum Chamædrys Linn. .

- flavum Linn.

- montanum Linn. . . . 68

- Polium Linn. . . . . . . 69

- scordioides Schreb. . . 40

- Scorodonia Linn. . . . 64

Thalictrum angustifolium Jcq. 40

- aquilegifolium Linn. ... . ivi

- minus Linn. . . . . . . ji

Theligonum Cynocrambe Lin. 42

Thesium alpinum Linn... . 70

Thlaspi Bursa pastoris Linn. 92

Thrincia birta Roth. ..... 55

Thymus capitatus $H o f f m$. et Link.

- Serpyllum Linn. . . . .

- vulgaris Linn.

Tilia grandlfolia Ehrh. . . . 25

Tordylium apulum Linn. . . 38

- maximum Linn. . . . . 36

Torilis helvetica Gmel. . . . 56

Tragopogon pratense Linn. . 54

Tribulus terrestris Linn. . . 81

Trifolium arvense Linn. . . . 80

- rubens Linn. . . . . . . . ivi

Trigonella Fcenum græcum $L, \quad 79$

Trinia vulgaris Dec. . . . . $\mathbf{3 8}$

Trisetum neglectum $R$. Sch, 59

Trochiscanthes nodiflorus $\boldsymbol{K}$. $\mathbf{3 7}$

Trollius europæus Linn. . . III

Tulipa sylvestris Linn. . . . 99

Tussilago Farfara Linn. . . . 51

Typha angustifolia Linn. . . 61

- Jatifolia Linn. . . . . . . ivi

Vaccinium Myrtillus Linn. . 106 - uliginosum Linn. . . . . . ivi Vaillantia muralis Linn. . . . 94
Valeriana officinalis Linn. . . 59

- Phu Linn. ..... . . . jvi

— saxatilis Linn. . . . . . . 87

— tripteris Linn. . . . . . 39

- tuberosa Linn. . . . . . ivi

Veratrum album Linn. . . 110

- nigrum Linn. . . . . . . ivi

Verbascum Blattaria Linn. . 83

- Lychnitis Linn. . . . . . . jvi

- sinuatum Linn. . . . . . . ivi

- thapsiforme Schrad. . 82. 83

- virgatum With. .... 85

Verbena officinalis Linn. . 64

Veronica Anagallis Linn. . . 84

- arvensis Linn. . . . . . ivi

- hederifolia Linn. . . . . . jvi

—officinalis Linn. . . . . 85

- serpyllifolia Linn. . . . 84

Vesicaria utriculata Lam. . 93

Viburnum Lantana Linn. . . 32

- Opulus Linn. . . . . . . . ivi

- Tinus Linn. . . . . . . ivi

Vicia dasycarpa Ten. . ... 82

- Pseudocracca Bert. .... ivi

- tenuifolia Roth. . . . . jvi

Vinca major Linn. . . . 88

- minor Linn. . . . . . . 89

Vincetoxicum officinale Moen. ivi

Viola tricolor Linn. . . . . 104

Vitex Agnus castus Linn. . . 32

Ulex europæus Linn. . . . . 79

Ulva intestinalis Linn. . . 118

Umbilicus pendulinus Dec. . 117

Urospermum Dalechampii

- Desf. . Dicroides Desf.... 54

Urtica pilulifera Linn. . . . 44

Xanthinm strumarium Linn. 96

Xeranthemum cylindraceum Sibth. Sm. .........

Zacintha verrucosa Garln. . 53 Zizyphus vulgaris Lam. .... 35 


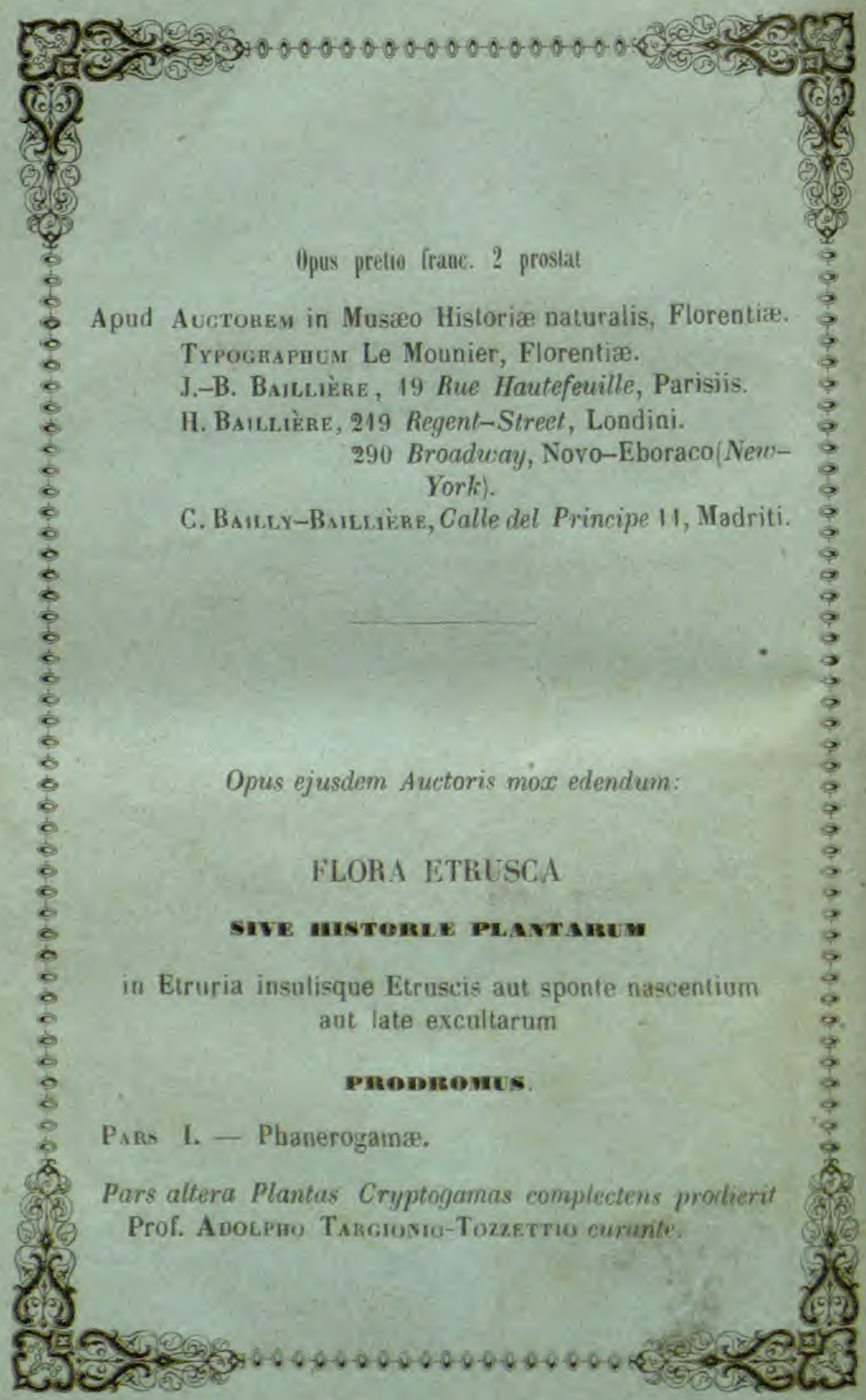

